\title{
MAKING USE OF SCENARIOS SUPPORTING SCENARIO USE IN PRODUCT DESIGN
}

\author{
DISSERTATION
}

to obtain

the doctors degree at the University of Twente,

on the authority of the rector magnificus,

prof. dr. H. Brinksma,

on account of the decision of the graduation committee,

to be publicly defended

on Wednesday, 17 November 2010 at 15:00

by

Irene Anggreeni

born on 6 February 1982

in Kudus, Indonesia 
This dissertation has been approved by:

prof. dr. ir. F.J.A.M. van Houten

promotor

dr. ir. M.C. van der Voort

assistant-promotor 


\section{MAKING USE OF SCENARIOS SUPPORTING SCENARIO USE IN PRODUCT DESIGN}

\section{PhD Thesis}

By Irene Anggreeni at the Faculty of Engineering Technology (CTW) of the University of Twente, Enschede, The Netherlands.

Enschede, 17 November 2010 
The promotion committee:

prof. dr. F. Eising

prof. dr. ir. F.J.A.M. van Houten

dr. ir. M.C. van der Voort

prof. dr. J.M. Carroll

prof. dr. ir. P.H. den Ouden

prof. dr. ir. P.P.C.C. Verbeek

prof. dr. ir. A.O. Eger
University of Twente, chairman and secretary

University of Twente, promotor

University of Twente, assistant-promotor

The Pennsylvania State University

Technical University Eindhoven

University of Twente

University of Twente

\section{Keywords:}

Scenario-Based Product Design, Scenario Creation, Scenario Management, Design Tool

ISBN 978-90-365-3087-3

DOI http://dx.doi.org/10.3990/1.9789036530873

Copyright (@) Irene Anggreeni, 2010

Cover design by Frederik Hoolhorst

Printed by WPS, Zutphen

All rights reserved. 
to my late father, Joseph Soenarjo Chandramoelja, and to those whose spirits are never broken in chasing their dreams 



\section{Summary}

With nowadays consumer products growing more complex in their functionality and more dynamic in their use situations, the design process needs to focus on the users to ensure a good usability. Taking into account the users and their use situations, designing such products tend to involve a large amount of design information and not uncommonly contradicting requirements to be dealt with by the design team. To answer this challenge, scenarios provide a low-cost, easy and accessible communication tool to explain design rationales, elaborate potential solutions, and discover where usability problems might arise. Scenarios are basically stories about people's experiences in using products. As concrete narratives, scenarios facilitate making explicit how users would use the designed product in their activities, allowing usability studies to be an integrated part of the design process early on and not as a detached post-design testing. The early integrated use of scenarios was in the design of computer systems/applications with the rise of Information Technology in the 1980s, in the process establishing the discipline of Scenario-Based Design (SBD). The later works are often tailored to each new domain, even to some extent to each specific case, making the developed SBD approaches not directly applicable to other domains. Within the context of consumer product design, the prior work in SBD is incomplete in that it has not fully addressed the particular characteristics of this domain. Therefore, the application of scenarios in consumer product design requires complementary research to identify the needs and necessary customizations, to create a more solid discipline of Scenario-Based Product Design (SBPD). Through collaboration with industry, this research aims to provide a practical guidance for applying SBPD in design practice.

This thesis presents an understanding on SBPD from the theoretical perspective as well as an application of this knowledge to support the practice of product design. Part one of this thesis, The Theoretical Review on Scenarios and Scenario-Based Product Design (SBPD), elaborates how scenarios can be a useful means to support the design process. The review on scenarios and SBD/SBPD reveals a lack of concrete guidance for creating scenarios and managing their use. While approaches, methods and tools to support scenario creation and management are available, they are either too specific for the particular domain or activity, not incorporating the dynamic use situations of the product, or not evaluated in a complete cycle of a real design process. In brief, the practical value of these approaches in the product design domain is still largely unknown. To fill in this knowledge gap, design practitioners are involved to complement the theoretical review, forming a mutual action and reflection between the researcher and the practitioners.

Part two, Action and Reflection, describes the cooperation with industry to identify practice-rooted problems and potential solutions concerning the application of SBPD principles. With an array of design methods and tools already being applied in practice, the design practitioners considered support for scenario creation and use in the form of a tool to be appropriate. Such a tool can combine and process the results from other used methods and tools, while at the same time also remains informal, encourages creativity and allows good interfacing with other activities in the design process. These characteristics of a support tool would give more flexibility and independence to the designers. The tool functionality was formulated to answer practical challenges by providing support in the following design activities: (1) the initial documentation of design information, (2) creating scenarios to make sense of the information, and (3) sustaining scenario uses as an integrated part of the design process. A conceptual design of the support tool was created using these criteria as guidance. Its evaluation was conducted with design practitioners by means of 
scenarios and mock-ups. As a result of this evaluation, the tool functionality has been refined, and later served as a reference for the implementation and evaluation of the support tool.

Part three, Implementation and Evaluation, describes the iterative development of an interactive prototype for the support tool. The interactive prototype was implemented as a web-based application. The future use of the support tool was discussed by means of scenarios and the interactive prototype. To represent the realistic target users of such support tool, the evaluations have been conducted with different types of design practitioners through focus group meetings. The mid-term evaluation involved two groups of target users, novice and experienced in using scenarios. The findings show that the support tool is suitable for the practices of designers both experienced and inexperienced in using scenarios. The evaluation of the final prototype compared the applicability of the support tool in the practices of two design companies, representing "small company" and "big company" cultures respectively. The support tool offers functionality that is applicable in both cultures. Nevertheless, its implementation generally needs specific adjustments for each particular practice. In the case of a "big company" context, the implementation would require a larger adjustment from both sides: more extensive tool functionality as well as changes in the practice or structure of the organization, and therefore a deeper commitment from the organization.

This thesis concludes with part four, Conclusions and Recommendations, which summarizes the key findings and the directions for future work. The contribution of this research is a practice-based approach to create, use and manage scenarios in a product design process. This research has revealed that the availability of support increases the willingness of design practitioners to use scenarios in their projects. The effort to create and maintain scenarios is considered justified as long as the scenario use is sustainable, and there is good interfacing with other activities in the design process. In providing concrete guidance for scenario creation and use, the support tool proposed in this research is most acceptable when it remains informal and encourages creativity. Eventually, future work could use the tool design and the recommendations provided in this thesis as the basis for making the support tool available to the design industry at large. 


\section{Preface}

It all began sometime in May 2006 when I discovered a PhD position on Academic Transfer. The initial assignment was to create a tool to generate scenarios to support the process of designing products, with a possibility to collaborate with another PhD project on applying Artificial Intelligence to make computers tell stories (by Ivo Swartjes, completed in April 2010). With my background in Human-Computer Interaction, familiarity with applying scenarios in interaction design projects during my master studies, and growing interest in the design of real physical products, I found the assignment a perfect opportunity, where I can use my knowledge and explore a new domain to apply it. I got in contact with my future supervisor, Dr. Mascha van der Voort, and in September 2006 I started my PhD at the University of Twente within the Laboratory of Design, Production and Management (OPM) of the Faculty of Engineering Technology (CTW). The project was initially funded as a part of SRO-NICE, and later transferred to IMPACT. Four years later, this thesis turns out not exactly about what the initial assignment was. A number of people I met during my PhD journey have generously shared their insights, which in turn shaped my approach and contribution within this research. I was given freedom to choose to focus on bringing scenario creation and use on a more practical level within the product design practice. To get here, I owe the help and support from many people. I therefore offer thanks to these people, without whom this thesis would never have existed.

First of all, my gratitude goes to my promoter Prof. Fred van Houten who made this PhD journey possible. I deeply thank you, Fred, for your trust in my quality and your full support that I could complete this thesis in time. Your warm yet firm guidance has motivated me and kept me going to achieve my goal. To my daily supervisor, Dr. Mascha van der Voort, I would like to extend my sincere thanks for never giving up on me and continuously helping me grow as a researcher. Mascha, you have been there from day one, with your tireless and unceasing support. Thank you for sharing your wisdom, which could always find solutions to any problem with tactfulness.

Many thanks go to my doctoral committee members who were willing to assess this thesis, provide feedback, and be part of the PhD defence ceremony. My special thanks go to Prof. John Carroll, whose work has inspired this research, for making the time to give comments and suggestions. I really appreciate your constructive feedback, which has put my understanding on this topic on a better perspective and eventually helped me to improve this thesis.

With the practical leaning of this research, I discovered the other half of the answers in collaboration with industry. For their committed participation in this research, I would like to thank all Indes members - Erik Woldring, Menno, Maurice Tak, and many others, who have shared their time, knowledge and experience in the various sessions I held, to help me understand the user-centered design in practice and later to evaluate my support tool. My gratitude especially goes to Willem Mees van der Bijl, who was always helpful in organizing my sessions and in providing significant input to my research, Chris van Dijk who helped me get familiar with FMEA and provided extra feedback for the evaluation, and Wilfred Teunissen who has shown genuine interest in this research and generously offered his insightful input.

In the later phase of my research, through the KWR project, I got in contact with Manel Leuverink from the Application Research Centre of Philips Drachten. This contact has allowed me to evaluate my support tool further with members of Philips ARC. I would like to thank the participants of my workshop at Philips, with whom I have had a lively discussion about my work and brought it in a different light. Especially to Manel 
Leuverink, many thanks for your support in organizing this valuable study. I really appreciate your warmth and sincerity in helping me in my research, and your encouraging words. I have also learnt about the differing design practices from Lilian Henze from P5 Consultants and the design practitioners who participated in the Usability Workshop in Delft, to whom therefore I extend my gratitude.

My colleagues at CTW/OPM and in particular at the group Use Anticipation in Product Design have not only shared their knowledge with me, but also fun moments together. I appreciate both work and play that have been constructive towards my research. My heartfelt thanks therefore for OPM-ers for being there to offer a helping hand when I needed your insights and feedback, and also for being spontaneous with the social activities we had as a group. To Mieke van der Bijl-Brouwer, whom I had the privilege to teach alongside in the master course Scenario-Based Product Design, I would like to thank you for inspiring me in the process of teaching. I would also like to thank Fjodor van Slooten, who has supported me in tackling some technical parts of Drupal. Also to Jan Willem Hoftijzer who has made me pick up running with his Horsthollers Bata-team initiative.

I spent a considerably large amount of my PhD time with the office mates at N-211, who thankfully made it easier for me to bear with my research when the going got tough. Your lighthearted jokes, whiteboard drawings, and funny impressions on various aspects of Dutch culture have often cheered me up. Thank you for being such cool office mates! To Frederik Hoolhorst in particular, you have been supportive in many ways these past years, that I feel honored to have you as my paranimf. My warm thanks also go to Bob Peeters, who has accepted my request to be my other paranimf despite his surprise. Bob, I seek you not with hours to kill, but with hours to live. Thank you for your friendship, and for being by my side during my defense.

While this PhD has been a large part of my life, thankfully there has been a constant reminder that there is life outside of it. I have been blessed with great friends and lucky enough to discover my passion for dancing. To my dear friends Sumy Bong, Novi Herawati Bong and Vivi Tjhin, we live our different lives apart, yet you understand and support me in the things I want(ed) to do, including the PhD that resulted in this thesis. I cannot thank you enough for our friendship. I am also grateful to have met Ella Meilianda during my stay in Enschede. To an extraordinary woman that has inspired me with independence and also family values, thank you! I would also like to thank Ingvar and Gunvor Wareborn, for the hearts that are always open to welcome me. I am grateful that our paths crossed years ago and that our friendship remains through all these years. To the friends I've been lindyhopping with, especially in Enschede - Rielle, Jolet, Dieter, Huub, Erik's, thank you and respect for all the swing outs, dips and pancakes! I also thank the beautiful souls I met in Eltamango, from whom I learnt to dance and be present. Especially to Ria Pabon, thanks for showing me some of your gems of wisdom. I could have written pages to thank you all, but dear friends, I think you understand my thankfulness for all the moments we share, even without a word written or spoken.

Last, but certainly not least, I thank my family who provides me with unconditional love no matter what I do, wherever I am. To my father, I remember your words that I have been holding on to since. I dedicate this work to you because, even without you telling me explicitly to go for a PhD, I think this is what you would have wanted me to be...

Enschede, 17 November 2010 Irene Anggreeni 


\section{Table of Content}

Summary vii

Preface $\quad$ ix

Table of Content $\quad$ xi

Part I Theoretical Review on Scenarios and Scenario-Based Product Design 1

Chapter 1 Introduction 3

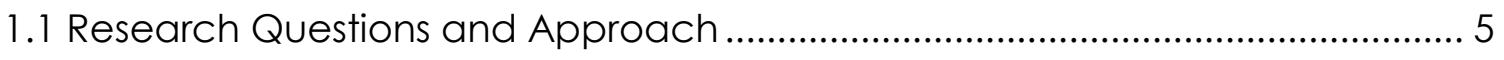

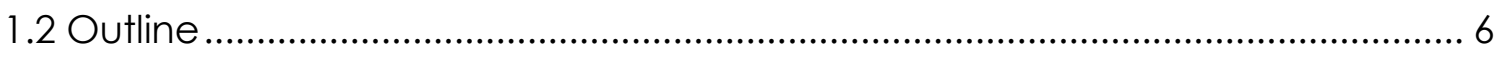

Chapter 2 Scenarios and Product Design 9

2.1 A General Introduction to Scenarios ................................................................. 9

2.2 Scenario-Based Design Process: A Theoretical Review ...................................... 12

2.3 Analysing the Benefits of Scenario-Based Design .............................................. 13

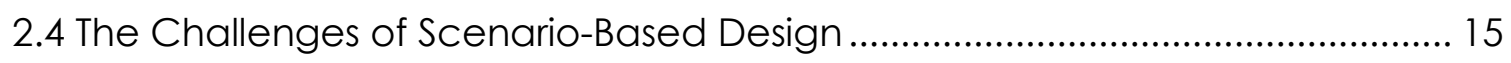

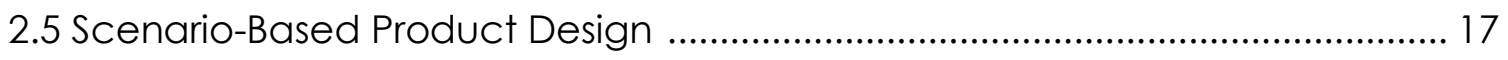

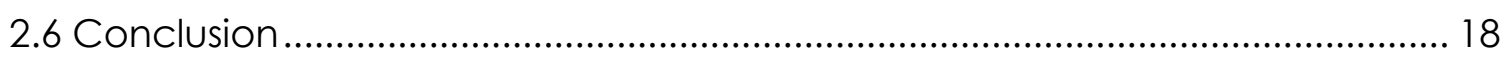

Chapter 3 Scenario-Based Design Researches 19

3.1 The Development of Scenario Use for Design ................................................... 19

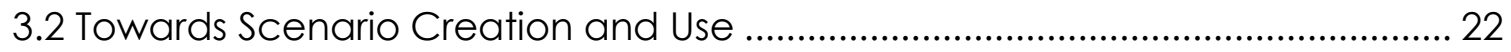

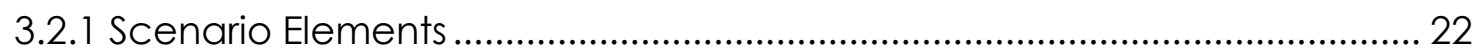

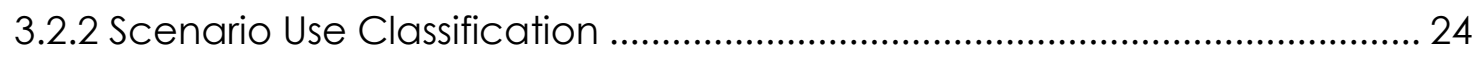

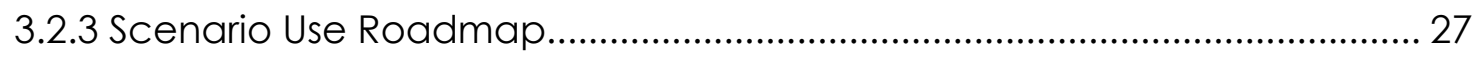

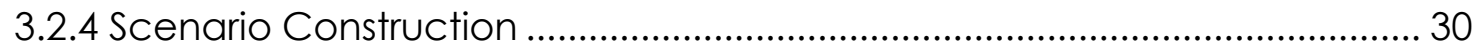

3.3 Lessons Learnt for Supporting Scenario Creation and Use ................................. 39

3.4 The Challenges: Revisited and Refined for Product Design Domain ............... 40

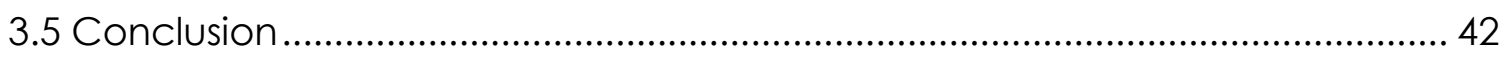

Part II Action and Reflection $\quad 43$

Chapter 4 Scenario-Based Design from a Practical Perspective $\quad 45$

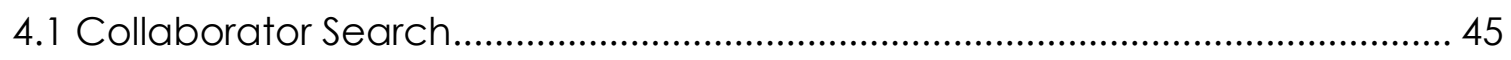

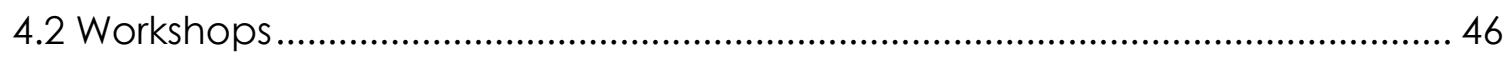

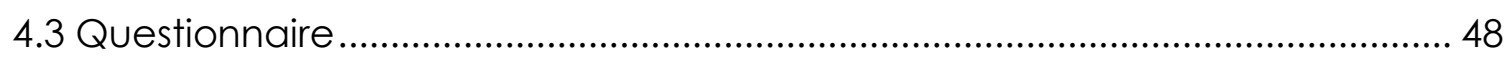

4.4 Scenarios in Product Design Practice ................................................................ 49

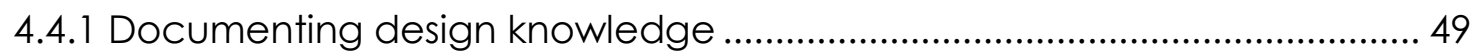

4.4.2 Proposing solutions and identifying requirements ..................................... 50

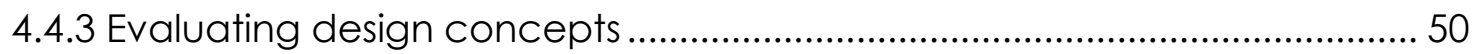


4.5 Criteria for a Framework for Scenario Creation and Use ................................52

4.5.1 Documenting design information as scenario elements ............................52

4.5.2 Creating scenarios to make sense of the information................................52

4.5.3 Sustaining scenario uses as an integrated part of the design process ......52

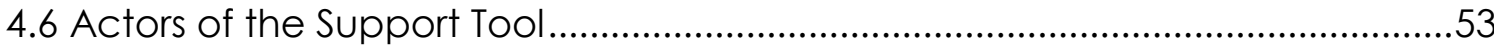

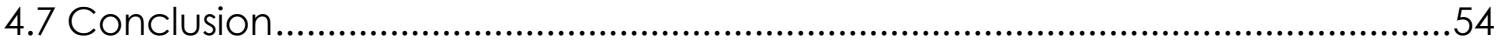

Chapter 5 Conceptual Design $\quad 57$

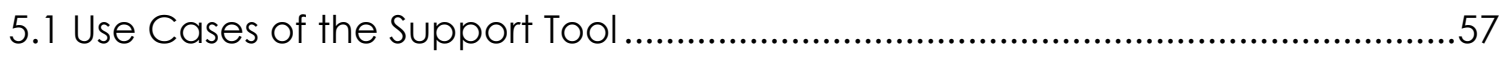

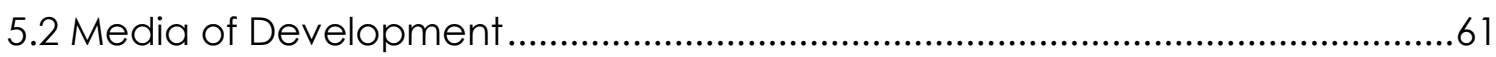

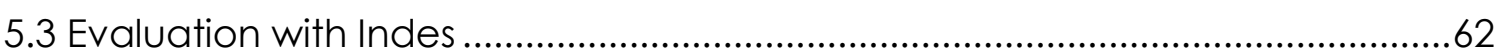

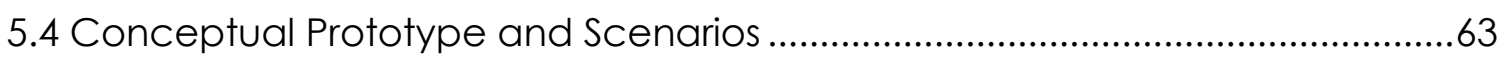

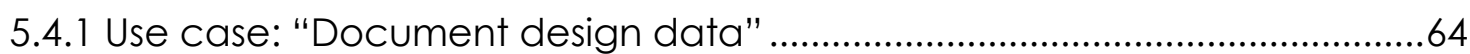

5.4.2 Use cases: "Create scenario" and "Create requirement" .........................69

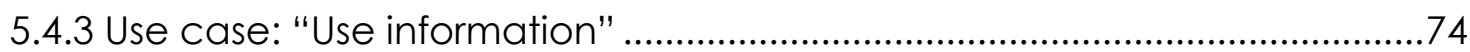

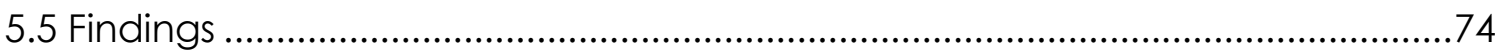

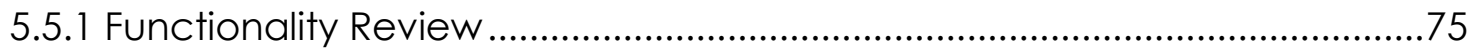

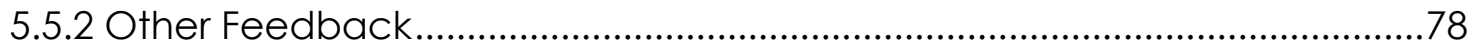

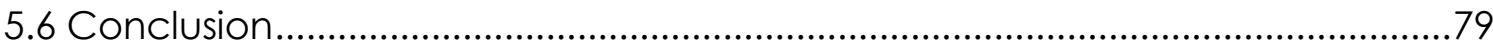

$\begin{array}{ll}\text { Part III Implementation and Evaluation } & \mathbf{8 1}\end{array}$

Chapter 6 Evaluation Setup and Implementation 83

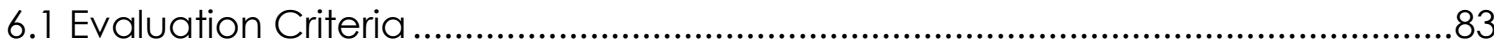

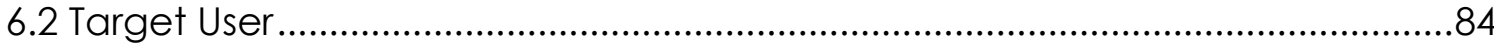

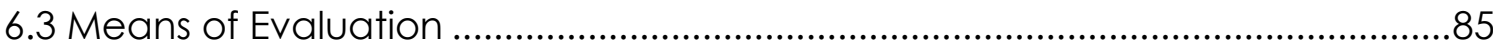

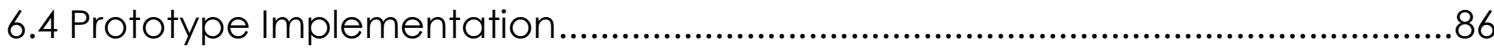

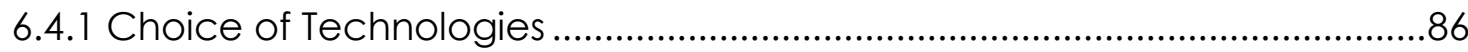

6.4.2 Drupal as a Prototype Development Platform ..............................................88

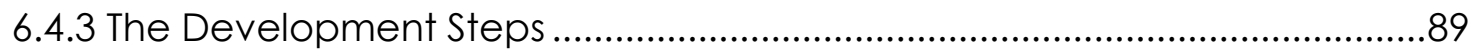

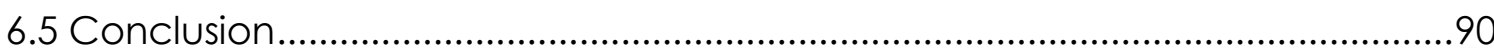

Chapter 7 Intermediate Prototype and Evaluation 91

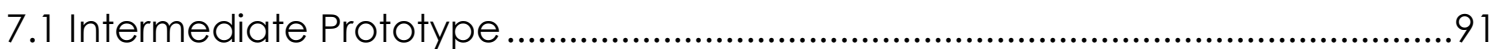

7.2 Evaluation 1: The Usability Workshop with General Designers (novice) ...........98

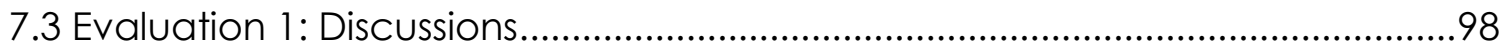

7.4 Evaluation 2: Focus Group Meeting with Indes Designers (experienced) .......99

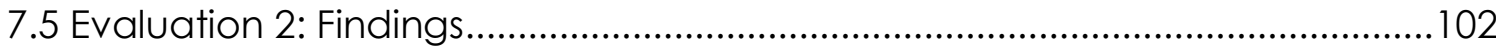

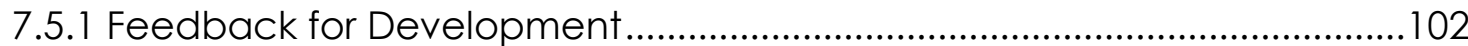

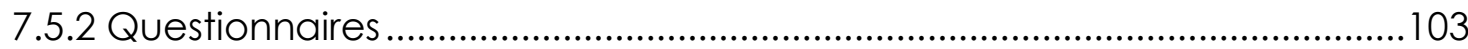


8.1 Use case: "Document design data" ................................................................... 110

8.2 Use case: "Create scenario" ................................................................................. 122

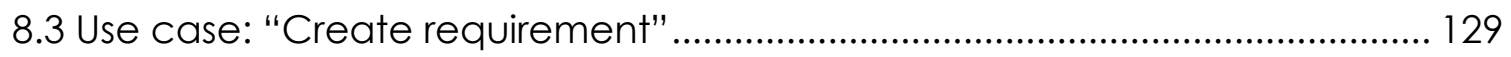

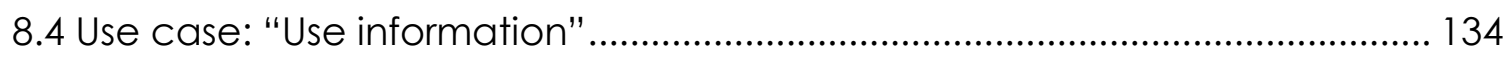

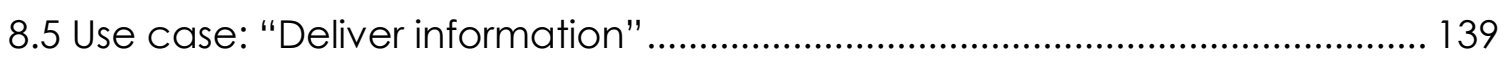

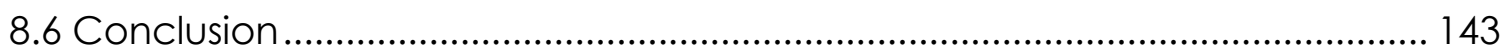

$\begin{array}{ll}\text { Chapter } 9 \text { Final Evaluation } & 145\end{array}$

9.1 Evaluation with Indes ("small company" culture) ........................................ 145

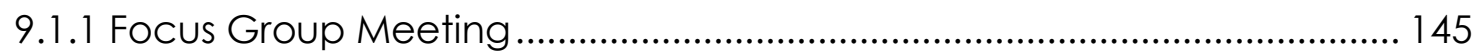

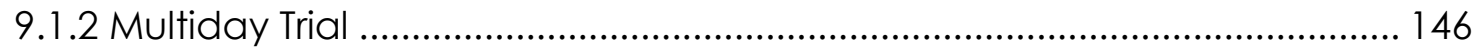

9.2 Evaluation with Philips ("big company" culture) ............................................ 146

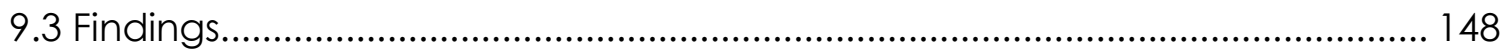

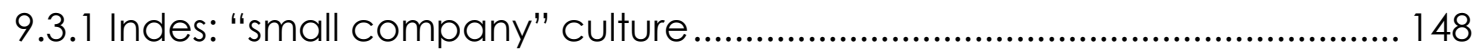

9.3.2 Philips: "big company" culture .................................................................. 150

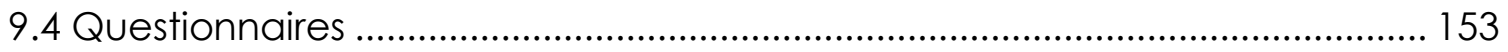

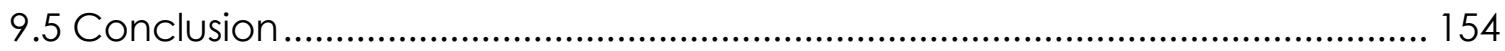

Part IV Conclusions and Recommendations $\quad 157$

Chapter 10 Conclusions and Recommendations 159

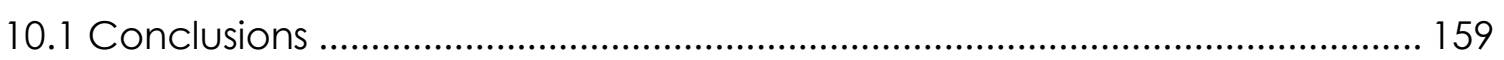

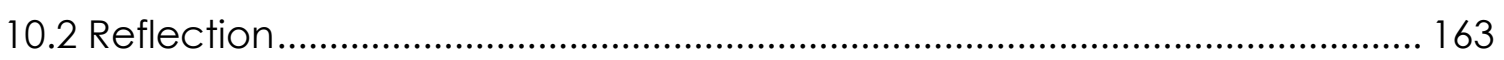

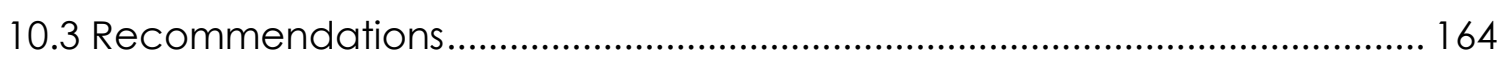

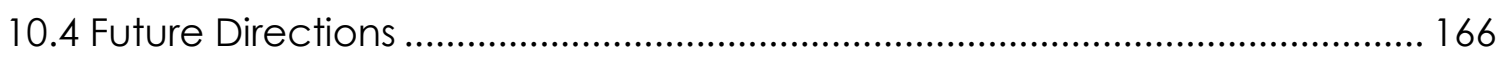

Appendices

Appendix 1 Explorative Workshops at Indes ......................................................... 169

Appendix 2 Explorative Questionnaire .............................................................. 171

Appendix 3 The Usability Workshop Program ....................................................... 181

Appendix 4 Evaluative Questionnaire (Intermediate and Final Evaluation) ... 183

Appendix 5 Intermediate Evaluation: Questionnaire Analysis.......................... 187

Appendix 6 Final Evaluation: Questionnaire Analysis........................................ 195

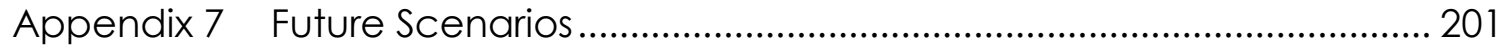

$\begin{array}{ll}\text { References } & 204\end{array}$

$\begin{array}{ll}\text { About the Author } & 209\end{array}$ 

Part I

Theoretical Review on Scenarios and ScenarioBased Product Design 



\section{Introduction}

Design, as an activity of creation, has been around since humans developed their creative skill to solve their problems. In the beginning, we needed simple solutions to address obvious problems; the functional requirements were clear. Therefore products could be designed to perform a set of functions which are direct solutions to problems, e.g. a knife to chop vegetables, a teapot to brew tea. Nevertheless, the continuous advancement of technologies has been creating more possibilities for products. The functional requirements for such products can be formulated only after their future uses are understood. One example of these technological advances took place decades ago in the early development of computer applications. The development of Graphical User Interfaces (GUI) in the 1980s has opened up more ways than just typing commands to interact with and operate software applications. The GUI has made interacting with computers easier and thereby computers more accessible to lay people. Think of how personal computers, and later equipped with the internet, have revolutionized our lives. Who would have thought then that we would need products such as webcams for conferencing or joysticks for playing games? To these days the trend continues. New technologies are waiting for a wide-range of applications in consumer products. For instance, a touch screen has been the norm for nowadays smart phones. Furthermore, it is now possible to interact with products using natural movements (e.g. the competing technologies Kinect, Wii Remote/MotionPlus, and Playstation Move) or even using thoughts (e.g. brain-computer interfaces developed by the competing Emotiv, NeuroSky and OCZ). Technology development is boundless; developers have the resources to build products with advanced functionality but are often uncertain what is required of the products. To start with, products need to offer at least marginal benefit to the users to be successful in the market (Cooper and Kleinschmidt, 1986), for instance by solving our problems and improving our lives. Therefore consumer product design will thrive better in the competitive market by shifting the focus to users.

Users can identify areas in their lives that may be helped or improved by products. However, as the products are becoming more technological, they also have concerns that it would be difficult to use these products. Jordan (1997) suggests that when a product increases in its functional complexity, usability should be prioritized over other dimensions (e.g. pleasure, aesthetics, and emotive aspects). A technically functional product would be simply useless if people do not understand how to use it. Usability is nevertheless broader than just designing for ease of use, and covers the overall quality of user-product interaction. Therefore the design process needs to take into account the users and the context in which they are going to use the product. This approach is generally referred to as User-Centred Design (UCD). A user-centred design process essentially gathers information about users (e.g. their characteristics and goals) and use situations early on in the design process, and then uses it as a reference to guide the process. While UCD concerns mostly design information from the user perspective, there is information from other perspectives to take into account in the design process. For instance, clients/producers might determine the product's unique selling points, specific materials, manufacturing methods, and other production criteria (e.g. time frame and cost). It may also be that experts in the particular technologies/domains are consulted for guidance e.g. on how to implement specific parts of the product. All in all, a design project often brings together different individuals from varied backgrounds with their specific knowledge. The design team needs to develop a solution that meets the 
interests of all stakeholders. It is not exaggerating to say that the success of a design project is heavily reliant on the communication between all parties.

Product design therefore requires a solid approach that integrates varied design information that covers users, settings, products and their interactions into a clear image of product use. Such approach should also support the communication of this image to various stakeholders. Scenarios are a good communication means that can potentially support these activities. Scenarios are basically concrete narratives that describe the hypothetical use of the product being designed. From them, designers can extract the functional requirements of the product. The early integrated use of scenarios was in the design of computer applications with the rise of Information Technology in the 1980s, in the process establishing the discipline of Scenario-Based Design (SBD) (Carroll, 1995). With the technology about to change the way people work immensely, scenarios are used to discuss and analyze how the new technology fits into their activities. The users are therefore empowered with the possibility to co-design, and not only asked to give approval to designs. This approach essentially uses the concreteness of scenarios to communicate problems and solutions explicitly. A simplified SBD process is illustrated in Figure 1.1. From users and stakeholders, designers get insights in the world as it is now with its needs and problems, as well as early feedback on their proposed solutions.

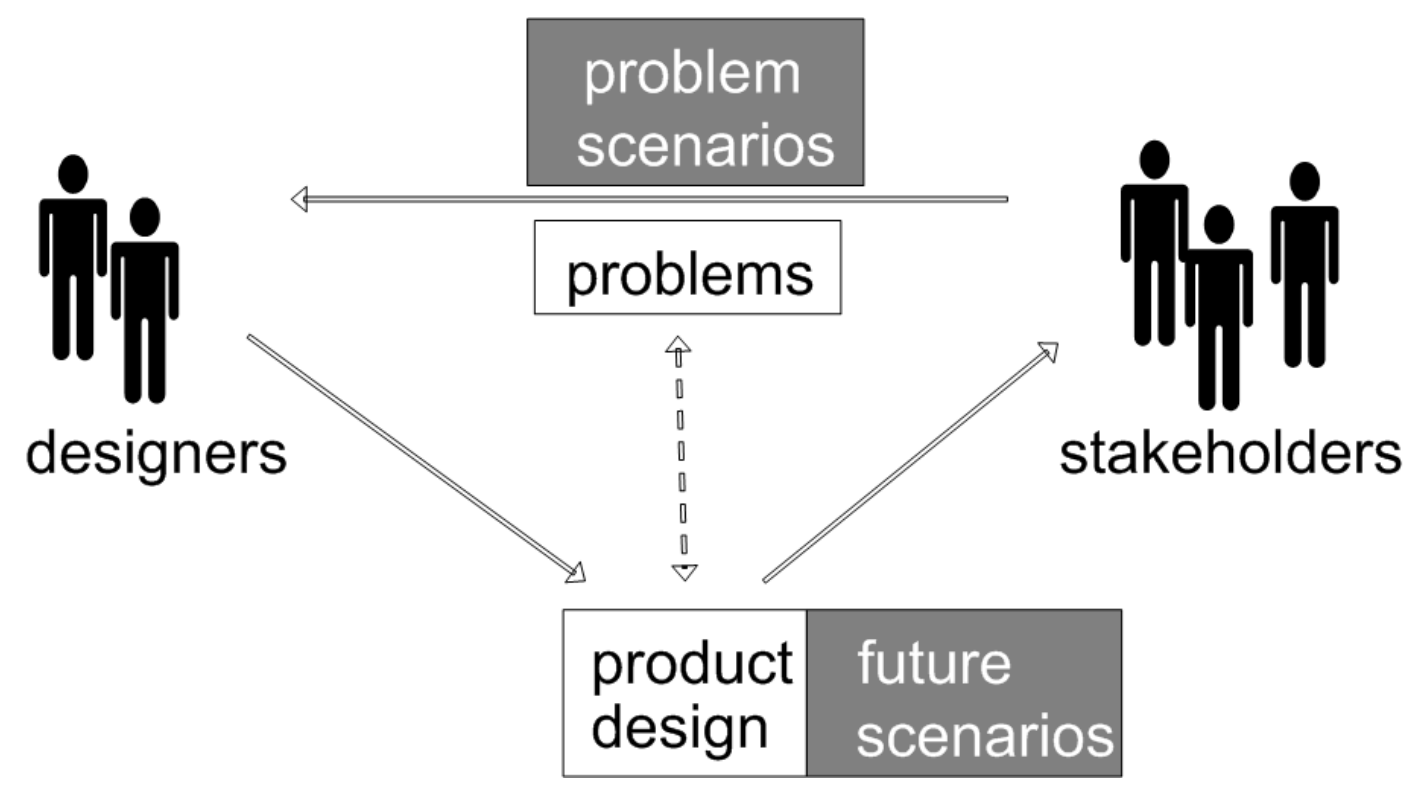

Figure 1.1: A simplified model of communication using scenarios in a design process.

The framework for SBD has been developed further in the area of interactive system design (e.g. Carroll, 2000b; Rosson and Carroll, 2002). Later work is mainly tailored to each new domain (e.g. Rolland et al., 1998b; Alexander and Maiden, 2004 in requirements and software engineering respectively), and to some extent to each specific case (e.g. Nielsen, 1990; Cooper and Reimann, 2003), making the developed SBD approaches not directly applicable to other domains. For application in the domain of consumer product design, the knowledge and experience from SBD needs to complemented and customized. Although scenarios have been used within product design (e.g. Moggridge, 1993; Fulton Suri and Marsh, 2000), they are not yet integrated in the design process and their application remains ad-hoc. Another reason is the different characteristics in the end-products of product design in comparison with the other domains. For instance, the early interactive systems to which the SBD was first 
applied, concern a more limited or predictable set of users, interactions and use situations. On the other hand, consumer products nowadays tend to be more portable, multi-functional and intended for a wider variety of users. These characteristics, referred to as Dynamic Use Situations (DUS) by van-der Bijl-Brouwer and van der Voort (2008), add complexities in designing consumer products. The application area of scenarios in product design, Scenario-Based Product Design (SBPD), will need to address these particular challenges.

Theoretically SBPD promises benefits to the process of designing complex products. To support its realization and apply it in practice, this research aims to provide practical guidance to SBPD processes by addressing the fundamental activities, i.e. creating scenarios and organizing their use in a design process. These backbone activities are essential for applying SBPD in practice, especially now that design information is typically growing in size and complexity. Nevertheless, they are very little supported by the existing approaches. These approaches mainly guide scenario use on a heuristic level and hardly concern the actual setup of using scenarios in a design project. Furthermore, the available scenario use exemplars from product design do not sufficiently cover scenario use in a whole design process, leaving the designers partly unsupported. Based on the existing knowledge in SBD, this research aims to provide concrete support for creating, using and managing scenarios for the particular domain of product design. Such support is expected to guide designers in effective and efficient use of design information within the product design process through the application of scenario-based design principles. Amidst many available approaches, methods and tools, it is most important that the proposed support does bring added value to the product design domain, improves the design process, and imposes the least possible burden with its application.

\subsection{Research Questions and Approach}

To achieve the aforementioned objective, first a deeper understanding on the Scenario-Based (Product) Design and scenario use in design is required. This understanding will become the foundation for the more practice-oriented research steps. To direct a coherent approach towards the objective, this research poses the following main questions:

RQ1. What makes the use of scenarios relevant in consumer product design?

RQ2. Why is further research in the SBPD discipline necessary?

RQ3. What activities in product design practice can be supported by the use of scenarios?

RQ4. How can another form of support be useful for the identified activities (RQ3) in a product design process?

RQ5. In which stages should the support be gradually actualized?

RQ6. For which industrial context will the support tool be applicable?

Finding answers to these questions requires a combination of literature and practicebased studies. To reflect the practical leaning of this research, insights from the design practice are used to inform, verify and evaluate the findings from the literature studies. For instance, while RQ1 and RQ2 are mainly approached through literature studies, the studies carried out to answer them are informed through contacts with industry. Also in answering RQ3, some practical areas are hypothesized first based on the literature studies, and then verified, informed and refined together with the industry. RQ4 is tackled by direct collaboration with the design practice to discover the form of support 
best suited in real setting. The coupling between theory and practice helps this research to focus on developing pragmatic support that brings added value to the design practice.

RQ5 concerns the practical implementation of the support tool, and is addressed in three rounds of development and evaluation:

1. Definition of requirements and functionality by means of workshops and explorative questionnaires,

2. Development of interaction and use of the support tool by means of mock-ups and scenarios, evaluated in a focus group meeting,

3. Development of interactive prototypes to evaluate the interaction, overall functionality, and technological feasibility for implementation.

Lastly, the evaluation of the support tool involves different types of designers as the potential users of the tool. The findings are analyzed to suggest the contexts which benefit most from the support tool, as an answer to RQ6.

In answering the research questions, it is chosen not to address the representation of scenarios. This thesis explores only the basic medium of scenarios, i.e. narratives. This constraint does not affect the course of this research as it focuses on supporting the process of creating, using and organizing scenarios, and not the ways to represent them.

\subsection{Outline}

Based on the research approach, this thesis is organized into four parts:

Part I. Theoretical Review on Scenarios and Scenario-Based Product Design

Chapter 2 introduces the subject of scenarios and Scenario-Based Design, acknowledging the benefits and challenges and why product design can learn from it.

Chapter 3 studies the approaches, including methods and tools, within the design research community to guide scenario creation, use and management. A scenario use roadmap is proposed to summarize the potential use of scenarios in a design process, and serves as a foundation for the next steps of action and reflection.

Part II. Action and Reflection

Chapter 4 describes the collaboration with industry to identify current practical problems that could benefit from the application of scenarios. A set of criteria for the support tool is formulated to address these support areas.

Chapter 5 presents the functionality and conceptual design of the support tool by means of mock-ups and scenarios. The functionality is evaluated and refined, and later serves as a reference for the implementation and evaluation of the support tool.

Part III. Implementation and Evaluation

Chapter 6 sets a plan for the prototype development and the evaluation with industry.

Chapter 7 describes the mid-term evaluation using an interactive prototype. Two groups of target users, novice and experienced in using scenarios, are involved in the evaluation.

Chapter 8 presents the final prototype of the support tool. 
Chapter 9 elaborates the evaluation of the final prototype involving practitioners from two design companies, representing "small company" and "big company" culture respectively.

Part IV. Conclusions and Recommendations

Chapter 10 reflects on the findings in this research and delivers recommendations for future work. 



\section{Scenarios and Product Design}

Design: communication makes or breaks it. Its success or failure largely depends on communication. An effective communication between all parties involved is therefore a requisite. For people with different backgrounds and skills to work together for a common goal, a medium is needed for them to communicate effectively and efficiently. Stories are inherent in this process, as it is natural for us human to communicate by telling stories. What we call scenarios in this research are basically stories with specific purposes to aid design processes.

\subsection{A General Introduction to Scenarios}

The term 'scenario' has long been associated with scripts for films or plays. It later emerged as a tool for strategic planning in several domains such as military, business, and policy management. Within these approaches, scenarios help planners to 'reperceive' the way the world works and to decide soundly for the future (Schwartz, 1996; van der Heijden, 2005). This thesis however focuses on scenarios that are used in the design domain. Henceforth, the term 'scenario' will only refer to scenarios that are created and used during design activities.

The early integrated use of scenarios for design purpose was in interactive systems design, which mainly took form as computer applications during the rise of Information Technology (IT) in the 1980s. As IT was going to change the work practice considerably, system designers were using scenarios as a process-description tool. This practice puts the process of using the new system as the end-result of design, as opposed to the software alone as the end-result (Kuutti, 1995). Carroll (2000b) coins the term 'scenariobased design' for such approach, which uses concrete narratives to discuss and analyse how the technology fits into people's activities. Similar to the interactive system domain, product design domain has also been researching and using scenarios and storytelling for acquiring user wishes and needs and for proposing design solutions (e.g. Moggridge, 1993; Fulton Suri and Marsh, 2000). However, the approaches in scenariobased design often suffer a similar problem as other design methods and tools being developed within the design research community in the last decades. As Dorst (2008) points out, methods and tools often ignore the content (the design problem and the emerging design solution), the actor (the designer or the design team), and the context in which the design activities take place. They focus too much on enhancing the efficiency and effectiveness of design processes, falsely claiming that the constructed models, methods and tools will be valid for every designer, dealing with every possible kind of design problem, in any situation. This leaves a room for this research to refine the scenario-based approaches for the product design domain while at the same time acknowledging and taking into account all four aspects of design activities (i.e. content, actor, context, process).

The fundamental function of scenarios is to make explicit the use situations of a product from the perspective of the users. By creating scenarios, designers understand their users better and become more aware of the usability aspects of their product. As a result, usability problems and potential solutions could be discovered and anticipated earlier. As explained in Chapter 1, usability concerns many more elements outside the product itself, among others the actor and his or her goal, the environment and the interaction between these elements. To deliver products with good usability, these elements need to be understood by and well-dispersed to everyone involved in the design process. It is not exaggerating to say that effective communication and 
exchange of ideas with end-users and stakeholders is the key to achieve usability. A design process should consist of techniques and methods to gather the necessary and relevant information to develop a useful (needed), usable (understandable) and desirable (wanted) product (Sanders, 1992). Designers need to know all important aspects of how their product is going to be used. The way to achieve this is by 'asking' users and stakeholders for their inputs. Notice that 'asking' involves an array of design techniques and tools because users often cannot formulate directly what they need of a product. The information that results from using these techniques and tools would usually cover details about the end-users, their dreams, wishes and goals. To ensure a good usability in the interaction between the users and the product, the design team also needs information about the context in which the interaction would take place. Such information is for example the environment in which the product is going to be used, the sequence of steps that normally occurs, and the unexpected events that could happen within this setting. When users and stakeholders start revealing stories about their personal or professional life (related to the product's context of use), frustrations with their job, wishes and expectations, and so on, it is a good indication that the designers have succeeded in carrying out the techniques and/or tools. As designers, we want to get into that point, where our users trust us as 'confidant'. Consequently, we also need a way to organize these otherwise unstructured stories, and to extract the necessary information as input to the design process. Scenarios are a suitable media to bring up the stories together into coherent pieces. By doing so, they serve as a tool to support the communication, reflection and evaluation while designing.

Like any good stories, scenarios should have a point. Throughout a design process, various scenarios can be created to serve different purposes. From user stories or data from executing design techniques/tools, scenarios immediately find a good use for summarizing and highlighting important design information. Creating and sharing such scenarios is a valuable investment in the early stage of a design process. The design team could then use scenarios to build domain knowledge about the users and the contexts of use collaboratively. Later on scenarios can be communicated to the endusers and stakeholders for early feedback. In the end of each design phase, scenarios will be useful for the final validation of the design concepts. Throughout the design process, scenarios will grow and evolve to accommodate the needs to communicate among the involved parties. Furthermore, scenarios also change in contents and representations to serve different purposes in a design process. Amidst the variety of scenario purposes in various design cases, efforts to get insight into the different ways of using scenarios have lead to classifications of scenarios (e.g. Nielsen, 1990; Nardi, 1992; Rolland et al., 1998a; Weidenhaupt et al., 1998; Anggreeni and van der Voort, 2008a), which will be discussed in Chapter 3. Despite the benefits, integrating scenarios into the design process also demands structure and organization. An important factor that helps creating coherence between the scenarios and other design artefacts is by making the scenarios as concrete as possible.

Being concrete aids a scenario to make the point across to the target audience. This means that different stakeholders with different domain knowledge should interpret it in an unambiguous way. This can be achieved by making the scenario elements explicit. In an instance of a use situation, there may be many factors that influence what happens. Additionally, elements that compose the use situation also matter to the unfolding of a scenario. The summary below provides a simplified overview of scenario elements:

- Actor is the personification of users. As the main element of scenarios, the actor performs actions and/or reacts to events which in turn create the storyline. 
- Actor Goal answers the question "what does the actor want to achieve by using a product?". Every scenario starts with this element. This goal gets a more complete context when built together with other elements into a scenario.

- Product is the tool or functionality that the actor interacts with to reach his or her goal. A product here can be existing (think of competitor products) or imagined (black-box concepts, partial functionality).

- Setting could be physical or non-physical. Physical characteristics of a setting include: location, level of noise, lighting, humidity, etc. Non-physical setting (or a precondition) can include: the actor's stress level, time pressure to perform an action, actor is distracted or tired, and so on.

- Action is a conscious step performed by the actor with an intention to achieve his or her goal.

- Event describes something that happens unexpectedly, for instance a sudden change in other scenario elements. The actor will react to an event by adjusting his or her goal and/or actions. This would influence how the scenario unfolds, or trigger new scenarios.

Where these elements fit together in a scenario can be explained briefly as the following. Every scenario involves at least one actor and at least one goal. When multiple actors or goals are involved, some are usually more prominent than others and are given more weights for considerations in design. Depending on the purpose of the scenario, some elements can be given more emphasis than others. Scenarios often make explicit the otherwise unexpressed information such as the actor's mental activity. The actor performs this mental activity to translate his or her goal into action and to make sense of what happens afterward. It is therefore important to express this mental activity for instance through the medium of scenarios. The sequences of actions and events - actions that actors do, events that happen to them, changes in the setting, and so forth, create the scenario plot. These actions and events may aid, obstruct, or be irrelevant to goal achievement (Rosson and Carroll, 2002).

Any design process aims to deliver useful, usable and desirable products that succeed in the market. While finding out the information related to scenario elements is not the end goal, such information will provide a solid basis to the design process. There are design methods and tools available to acquire this necessary information, which can be from the perspective of current situation as well as imagined future. Both are complementary, with the designer seeking the middle ground to develop a feasible design that addresses the user needs and wishes. For instance, focusing on the current situation could reveal users' needs, problems and dissatisfactions, which lead to insights on what to improve. On the other hand, an orientation to the future helps capture the dreams, hopes and desires of the users, which inspires solutions. While scenarios are in essence narratives, they can be represented in different media such as storyboard, movie, or roleplay. This flexibility allows scenarios to easily accommodate various design information that come from both perspectives. Furthermore with explicit elements, it is easier to keep track of what could be changed in a scenario and what the consequences would be. For example, by changing one or more of its elements by e.g. a new setting, a new tool or a new set of actions, a current scenario could be transformed into a future scenario. Such scenario depicts a future as imagined by the designer and could be used to evaluate the proposed solution with users and stakeholders. The scenario elements as described earlier are a basic guideline into the inside of a scenario. The elements can be decomposed further into more specific parts and characteristics. Furthermore, they also relate to one another and create a network of influences (see Figure 3.4). 
This introduction section gives a common perspective on scenarios and their use in design on a general and theoretical level. There are definitely more uses of scenarios that have been explored in design practice, as will be addressed in Chapter 4. A design process, no matter the techniques, methods or tools that compose it, could make use of scenarios as its integral part. However, due to the different characteristics of products, target users, working cultures, designers and design teams, scenario-based design cannot be approached by prescribing a step-by-step methodology. Nevertheless, a more concrete guidance to scenario-based design will contribute to both the research domain as well as industrial practices. To reflect this situation, the definition of scenario-based design is used throughout this thesis:

Scenario-based design is a common denominator for techniques that apply scenarios to bring actors, products, environments and their interactions into harmony.

The scenarios, either as narratives or represented in other media, function as the glue that holds information pieces together. Aligned with the definitions proposed by various authors (Nardi, 1992; Carroll, 1995; Carroll, 2000b), this thesis considers the definition below sufficient within the context of this research:

Scenarios are explicit descriptions of the hypothetical use of a product.

This hypothetical use, as mentioned earlier in this section, can be from the perspective of current situation as well as imagined future.

Summarizing, this section has introduced the general background about scenario use in a design process and the role of scenarios in the approach. An overview on the elements and inspirations for scenarios has been addressed. Finally, a redefinition of scenario-based design and scenario is proposed to provide a common ground for this thesis. Deeper into the subject, the following section will elaborate how scenario-based design and scenarios relate with design processes at large.

\subsection{Scenario-Based Design Process: A Theoretical Review}

Within industrial design there have always been the two dichotomies of technologypush and market-pull. Translated into the designing activities, design projects may take on different natures that the designers have to adjust their strategies to. Kruger and Cross (2006) study the strategies that product designers may take on to solve a design case. The most prominent ones are problem-driven and solution-driven. Using problemdriven approach, the designer focuses on defining the problem and only uses information and knowledge that is relevant to solving the problem. When the problem is (re)defined, finding a solution follows as soon as possible. This problem-driven approach is natural within the market-pull dichotomy. In solution-driven design, the designer focuses on generating solutions, and less on defining the problem, which results in the problem often being reframed to suit an emerging solution. Information is only gathered when it is useful to further develop a solution. This solution-driven approach is suited in the technology-push dichotomy. While a solution-driven strategy tends to encourage high creativity, a problem-driven design strategy tends to produce a better balance in both overall solution quality and creativity. Understanding a design problem largely involves understanding the process that causes and influences it.

These problem- and solution-driven perspectives correspond well to the perspectives from the early days of software engineering. Floyd (1988) introduces product-oriented and process-oriented perspectives in software development. A product-oriented perspective, which has been established earlier, regards software as a product standing on its own, thus allowing software requirements to be predetermined. On the 
other hand, a process-oriented perspective considers software in connection with human learning, work and communication, within a dynamic world with changing needs. A process-oriented solution has a greater chance to integrate into the world of work. On the other hand, a product-oriented perspective could have inspired innovative solutions that offer opportunities to unknown users. The problem- and solution-driven strategies from product design are comparable to the process- and product-oriented respectively. Both perspectives should coexist in time, complementary to each other.

Striking a good balance between the differing perspectives is the essence of design as an activity of creation. To carry out this strategy, a design team employs a variety of methods and techniques. For instance, Jones (1981) presents a compilation of design methods to explore and understand problem and solution space in a design case. This compilation has two principal common features: formalization and externalization (Cross, 1994). The first one, formalization, deals with widening the approach in problem and solution space, and usually also creates a basis for further research. Within a huge and complex problem space, there is a risk that some important elements are overlooked, which is why formalization methods and tools are helpful. On the other hand, externalization helps designers to express their design thinking into charts or diagrams and the like, to release their memory from these thoughts, and therefore give more space for creativity. Externalization also deals with communicating design ideas with others involved in the design process. Both characteristics are present in the variety of design methods, and are complementary to each other. Designers have a set of methods to perform the formalization and externalization of their thinking. Yet connecting and organizing the results of both processes still presents a challenge. SBD proposes scenarios as the main tool to formalize and externalize the design thinking, which additionally helps the communication in the design process.

Scenario-based design relates well to the bigger perspectives on design. Such a connection shows that scenario-based design is not a solitary methodology that a design team either has to take or leave it. Instead it proposes the use of scenarios to improve different aspects in a design process, as will be elaborated in the next section.

\subsection{Analysing the Benefits of Scenario-Based Design}

The introduction section has delivered the message that using scenarios offers benefits to a design process. To understand why scenario-based design is beneficial, this section discusses the properties of scenarios and the design activities that involve them.

Storytelling is inherent within various communication aspects of a design process. The results are often impromptu stories which are not necessarily orderly. Scenarios wrap them up to highlight key issues in design, and are generally more organized and presentable than user stories. The scenarios can be based on either the current or future situations. In future-oriented scenarios in particular, product solutions can be described even when these solutions are still rough ideas. This allows quick and early evaluation without a design team having first to build elaborate or expensive working prototypes. With a minimal early investment, the design team does not get caught up in premature commitment just because it has spent a large amount of resource on a particular design direction. Furthermore, scenarios allow various media of representation to adapt to the audience. For instance, Carroll (2000b) recognizes prototypes, storyboards, videos and rapid prototyping tools as elaborated form of scenarios. By using lay-people language and flexibility in medium of representation, scenarios are fit as a medium for people of different backgrounds and disciplines to communicate and work together in a design team. 
Carroll (2000a) elaborates the characteristics of scenarios and analyzes how they answer technical challenges in information system design. Figure 2.1 shows an overview of the scenario characteristics and the related design challenges. This overview is also relevant to product design because scenarios maintain similar characteristics independently from the process and end-products (i.e. software system versus tangible product). Carroll (2000a) identifies the following characteristics:

- Scenarios are multi-faceted. Scenarios afford multiple views and different levels of interaction. They can be used for instance to describe the multiple views of different stakeholders in the project. As a result, it is easier to find out the consequences of a particular scenario for the different stakeholders. Furthermore, a scenario can also reflect the stage of design by describing an interaction on the appropriate level. From here on, the stakeholders can get an idea about the design maturity, whether is still ideas, rough concepts, or detailed specifications ready to be built.

- Scenarios afford simultaneous actions and reflections. Scenarios help developers to balance their design action and reflection, without the action obstructing the reflection and vice versa. The action of proposing solutions can be performed through constructing scenarios. The resulting scenarios provide developers immediate and concrete hypothetical use situations to reflect the solutions on. As opposed to continuously performing actions (i.e. synthesis) and reflecting on the solution only in the end, the possibility to perform actions and reflections in smaller iterations reduces the risk of unfitting solutions.

- Scenarios are at once concrete and moldable. Scenarios are concrete in the sense that they represent one instance, thus a unique interpretation, of the open-ended design situation. While products often deal with many and uncommonly complex use situations, designers could be overwhelmed by the information that explains these situations. It becomes important to narrow down the information and fix a few concrete scenarios that are most significant to start with designing. Scenarios are also flexible. They can be easily revised when the interpretation is found to be false, or elaborated when new details of the situation are encountered.

- Scenarios can be abstracted and categorized. Scenarios easily grow as the design progresses, as new insights are discovered. To start designing a product, scenarios need to cover the important use situations, and need not be exhaustive. Later on, as new insights as discovered, new scenarios can be added and existing scenarios can be improved. The collection of scenarios can be abstracted and categorized to create a structured learning source of the design domain. Such knowledge will be reusable for other design projects with similar characteristics. For instance, known safety standards (e.g. medical equipment, electronics, etc) are built upon critical scenarios that have been frequently encountered and gathered throughout years of practice. Using scenarios as its medium, the domain knowledge becomes more accessible. From here, a design team could explore the possible ways to apply a scientific knowledge or technological breakthrough in the design. From a different starting point, future-oriented scenarios could encourage the development of specific science or technology by justifying the needs for it (e.g. ISTAG, 2001).

- Scenarios encourage participation from users and stakeholders. Scenarios anchor design discussions in the (work) processes, where the users and stakeholders are the domain experts. The users, who are going to use the product, should have an active role in designing it. This approach is referred to as participatory design, and is based on a democratic ideal that everyone should have the right to participate in shaping the decisions concerning his or her life (Ehn, 1993). Furthermore, the participation of skilled users in the design process can contribute to successful design that results in 
high quality products. There exist participatory methods to involve users and stakeholders in design activities (e.g. Muller et al., 1993). Scenarios are inherent in any of these methods, as the common language between designers, users and stakeholders. Using scenarios, everyone has a means to communicate readily: designers could propose solutions by telling scenarios, and the users and stakeholders could verify the solutions right away or propose other ideas by telling different scenarios.

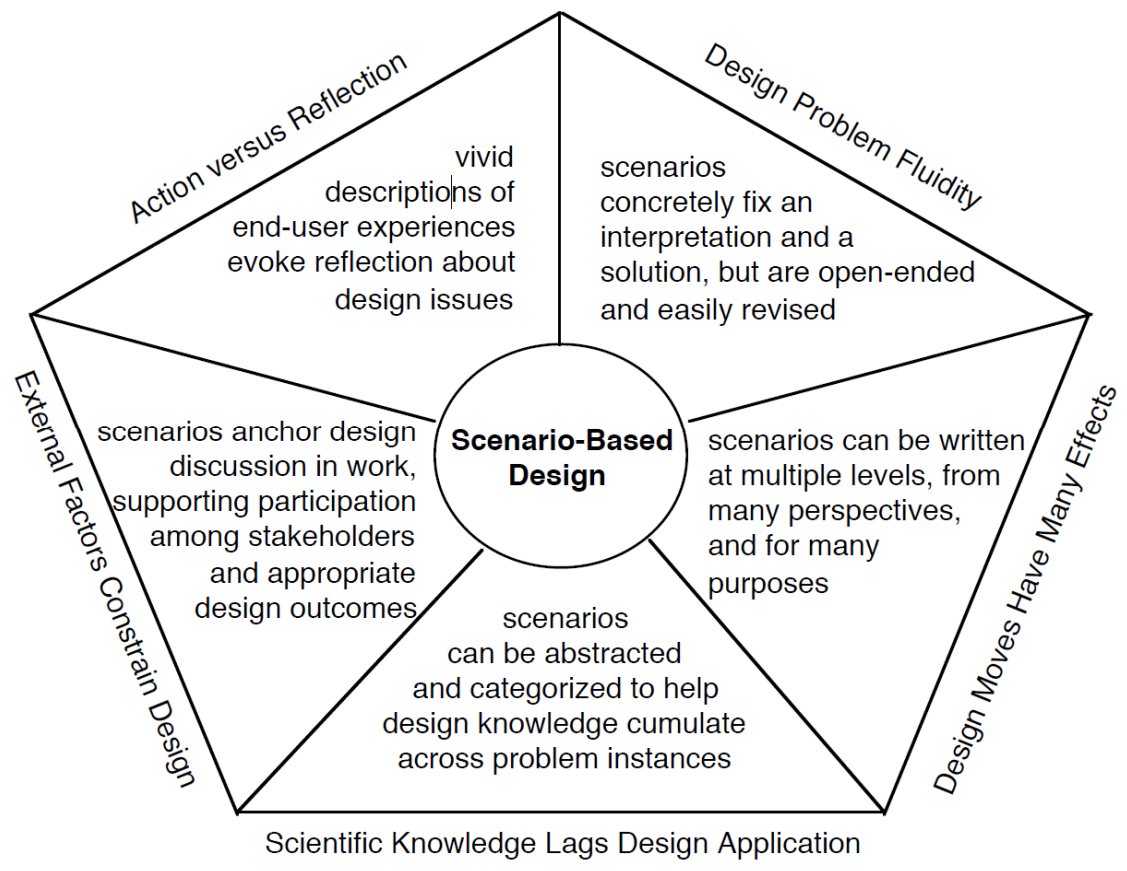

Figure 2.1: The key characteristics of scenarios that answer technical design challenges (Carroll, 2000a).

The characteristics of scenarios as described above promise benefits in a design process. Carroll (2000a) has elaborated them to point out to the design field why scenario-based design is a discipline worthwhile for further research. These promised benefits however, can remain uncovered potentials without answering how scenariobased design can be applied in practice. While abstract theories are sufficient to explain the essence of scenario-based design, its application will first require a deeper understanding of all aspects in design activities (i.e. content, actor, context and process). These aspects have not always been addressed in the theories and approaches of scenario-based design. This situation therefore poses some challenges in applying it.

\subsection{The Challenges of Scenario-Based Design}

SBD as a framework is intentionally non prescriptive, and consequently, its application needs to be tailored to each domain or case. The developed approaches in SBD seem to answer specific design questions, and therefore are not directly useful to other design questions that rise from different situations. For instance, the application of scenarios in product design still requires complementary research to address the knowledge gap pertaining to the domain. Furthermore, scenario-based methodologies are often formulated from non-commercial research. This could mean they are missing relevance with the aspects of practical, real life design. Exemplars of scenario uses from design practice, on the other hand, mostly cover only small parts of the design cases or provide limited explanations/rationales. Consequently design practitioners are still mainly unguided in setting up and conducting scenario-based design. Design teams 
will always need to adjust their approach and be eclectic in planning, implementing and evaluating scenario-based design according to their own situations.

In user-centred design, the design activities tend to involve various stakeholders. Furthermore, the focus on usability also includes various aspects of use situations that need to be taken into account. Altogether they contribute to the complexity of a design project. The resulting design data are usually large, and therefore require further processing to be useful in the design activities. While scenario building helps the process of analyzing the data, it also presents challenges in identifying and constructing scenarios that are meaningful to the design process. The challenges of bringing scenario-based design into practice can be explained as the following:

- First of all, custom-fitting scenario-based design to a particular design practice/project requires some investment, and design practices are often not ready to do so. The added values of using scenarios in the design process need to be identified and be considered against the currently used approach. To be able to evaluate the added values, the purposes of creating and using scenarios throughout the design process need to be clearly defined beforehand. This leads to more practical questions such as: "What types of scenarios should be created?", "What information should be included in the scenarios?" or "How to best represent them?". Without having a framework for scenario-based design, a design team would find it difficult to measure the efficacy of a scenario-based approach.

- Related with the previous challenge, the scope of scenarios in the scenario-based approach needs to be defined, and there is very little guidance for this. This challenge concerns for instance, what aspects of use situations should be covered in scenarios, or which of the scenarios should be prioritized in case a compromise is needed in making design decision. Scenarios should aim to focus the otherwise overwhelming design information. Defining the scope therefore helps to avoid too many scenarios that do not contribute much to the design process.

- Relevantly, a scenario-based approach needs to integrate available design methods and tools to get the necessary information for scenario building. There is very little overview on how existing design methods and tools can be a part of scenario-based design. Having such overview would help a design team to also solve the first challenge, to custom-fit scenario based design to their practice.

Design practitioners attempting to implement scenario-based design in real life projects often face those challenges. The existing knowledge on scenario-based design cannot be simply and directly applied to new domains or cases; it always needs to be tailored to the situation at hand. Furthermore, the currently available scenario-based methods and tools may not be relevant with the present needs of design practice (as will be discussed in Chapter 3). There is no uniform solution to bring scenario-based design into practice. Nevertheless, design practitioners will appreciate more support and guidance to help answer the identified challenges above.

These challenges are also recognizable in the product design domain. Furthermore, the more complex nature of designing tangible products amplifies these challenges. This research focuses on scenario use in the product design domain, and will direct the development of the support towards the needs in this domain. The next section will elaborate the product design domain as an application area of scenarios, within a discipline referred to as scenario-based product design. 


\subsection{Scenario-Based Product Design}

The characteristics of scenarios bring advantages in answering complexities within product design projects. The complexities could be for instance due to the composition or size of the design team, the different disciplines involved, the characteristics and diversity of the users and stakeholders, or the nature of the product itself. The vast amount of complex and interrelated information in product design often results in conflicting design requirements. Scenarios could make explicit use situations which give concrete foundation on which the conflicting requirements can be analyzed. This benefit is intensified with the trend of nowadays products that are becoming more intelligent. While more consumer products integrate software components, the opposite is also true, i.e. software products becoming more tangible. Technology development has allowed a more natural interaction between users and software products, as observed for instance in entertainment (e.g. Microsoft, 2010; Nintendo, 2010) or design industry (e.g. Wendrich et al., 2009). With these new possibilities, products are becoming more accessible to various users, each of whom can have multiple goals and contexts in using them (see Figure 2.2). Brouwer and van der Voort (2008) refer to the characteristics of such products as having dynamic use situations. A design team employs methods and tools to get insights into the dynamic use situations, and often ends up receiving a large amount of information. By highlighting the most significant use situations first, scenarios could provide directions for the design team to get started without being overwhelmed.

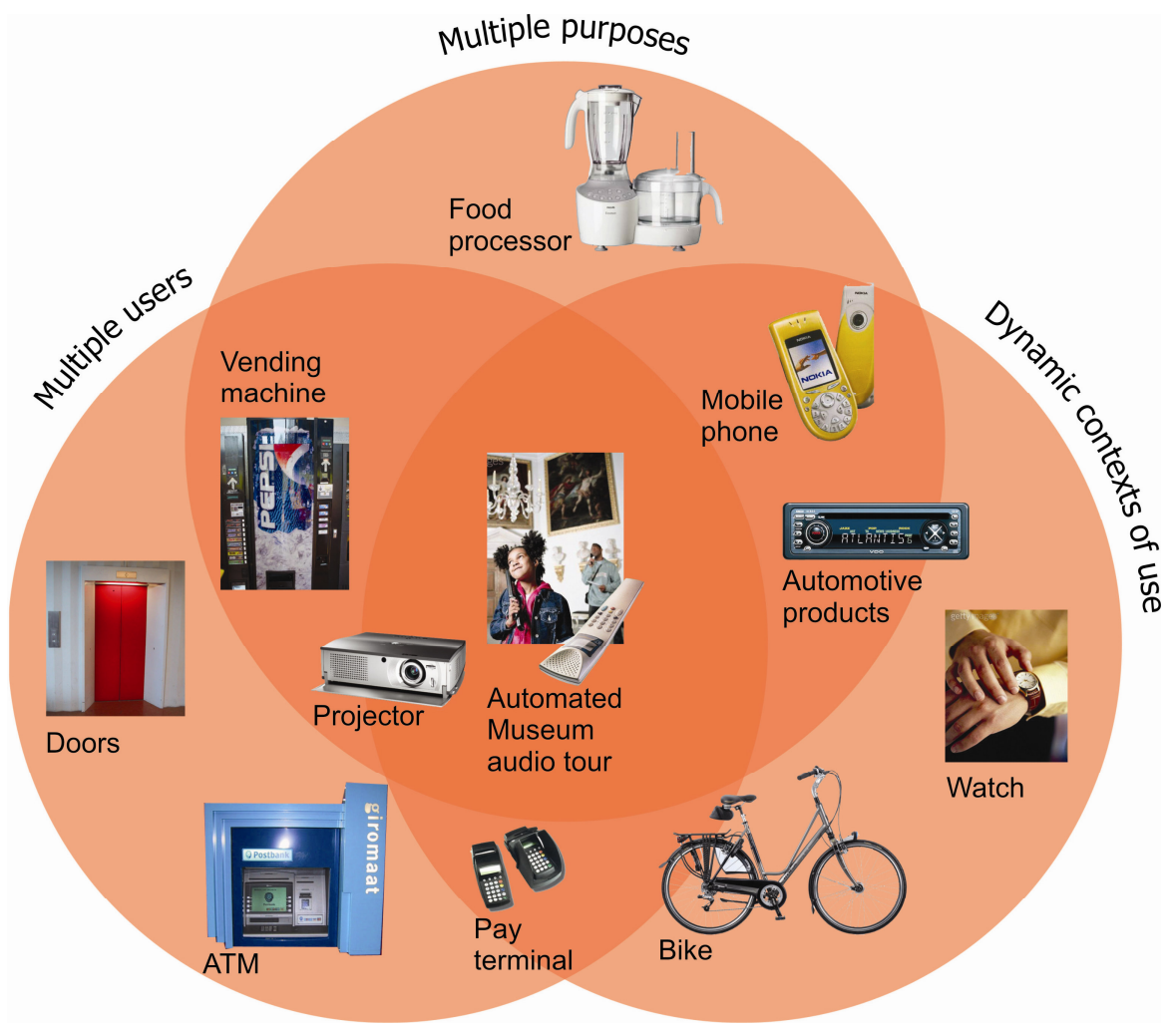

Figure 2.2: Dynamic Use Situations illustrated; example of products with varied users, multiple functions, and dynamic contexts of use (van der Bijl-Brouwer and van der Voort, 2008).

Motivated by the merging trends in tangible products and software/systems development, this research combines approaches from both domains. Though initial scenario-based approaches stem out mainly from interactive system design, consumer 
product design is continuously merging these existing approaches into a more holistic scenario-based product design. There are however some points for extra attention due to the difference between the two areas. The initial work on SBD was mainly applied in the development of early interactive systems, which have different characteristics from tangible products. These early systems, as in computer applications, concern a more limited set of interactions and use situations. Furthermore, they are often designed for a specific type of users, with known characteristics, skills and level of experience. For example, a web-based application is designed to be used by a group of office workers to manage projects. It could be assumed that the users (i.e. the office workers) are generally knowledgeable about computers and experienced in using web browsers. Such application has a closed set of interactions (a computer with mouse and keyboard) and is usually used in a static environment (i.e. office or corporate setting). Today's interactive systems are far more complex, rich, dynamic, as well as more personalized (think of iPad, or smart phones with Wi-Fi and GPS). Their characteristics are merging with those of the more tangible consumer products. While the Dynamic Use Situations (DUS) mainly characterizes the latter products, it is also becoming more common in current interactive systems. This situation demands reinforcement of the existing approaches with knowledge specific to the dynamics of nowadays products. Scenario-based product design allows integrating a variety of design methods and tools to create a more holistic approach covering the dynamics of product use.

\subsection{Conclusion}

This chapter has introduced scenario-based design, its benefits and challenges. Summarizing, scenario-based design can be applied in design processes at large. In any design process, scenarios and their flexible representation can be the means to combine the multiple aspects of product use, which aids understanding, communication and reflection. This role of scenarios becomes even more prominent in complex design projects, which might have many aspects in the product's use situations and not uncommonly conflicting requirements. Support is still required to realize the potentials of scenario-based design within consumer product development, which this research aims to provide. In developing such support, this research strives in acknowledging and understanding all four aspects of design activities within scenariobased design processes (i.e. content, actor, context, and process). Before proposing any solution, a comprehensive study is taken to learn from what has already been done in this field, in particular regarding the creation, use and management of scenarios. Chapter 3 delivers the results of the study. 


\section{Scenario-Based Design Researches}

Chapter 2 has elaborated why support is still needed in applying scenarios, especially in the product design domain. A practical area to look at is the creation, use and management of scenarios. There exist earlier works that describe the attempts to explain the nature of scenario uses in different design processes. This chapter will look them to gain insight on how scenario-based design has developed and to understand the motivation for the discipline. To inform this research about scenario creation, use and management in design, several topics are covered. These topics are a.o. the constructing elements of scenarios, the classification of scenarios based on their purposes, and lastly the available approaches towards scenario creation, use and management. A reflection is conducted to relate the findings from the literature studies with the product design domain. This chapter is concluded with a set of focused research questions which will involve the design industry to answer.

\subsection{The Development of Scenario Use for Design}

As explained in Chapter 2, scenario-based design was initially applied within the field of interactive systems design. With the systems becoming more personal and more available to many users, the need for usability in this field was becoming apparent. Usability studies are recognized to be an integral part of the design process, and not only be applied as a detached post-design testing (Carroll, 1995). The end-users should be empowered with the possibility to co-design, and not only giving approval to the final design (i.e. a computer application). The application of the then new information technology created new practices, and consequently also new problems that urge solution redesign. The design affords more tasks to be performed by users, prompting further redesigns. Within this task-artefact cycle, a final design cannot be known for certain (Carroll, 2000b). Scenarios can help designers cope with this uncertainty. They allow capturing situations ranging from the rich context to the detailed low-level interaction. Kuutti (1995) suggests a complementary use of contextual and application scenarios. "Rich" contextual scenarios describe the work practice in which the service is going to be used. On the other end, "narrow" application scenarios are describing the services of the system being built. A transformation deals with embedding systematic application scenarios into proposed contextual scenarios. Kuutti (1995) uses an abstraction to show the three worlds involved in IS design (see Figure 3.1). While the system designers work mostly in the conceptual world of abstraction and logic, they need a means to access the processes in the real world of work. Scenarios become the tool to describe the top two worlds and bind them together.

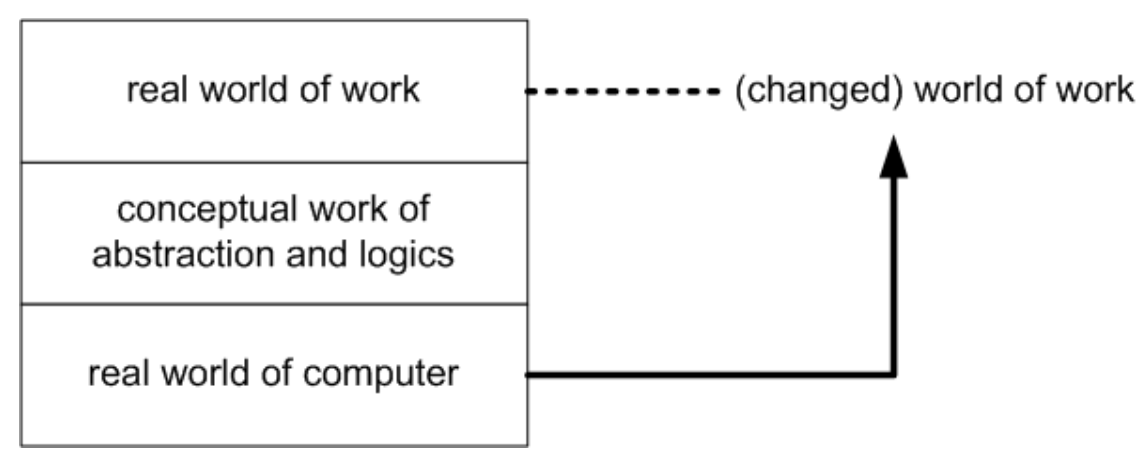

Figure 3.1: The three worlds of system design (Kuutti, 1995). 
Scenarios within interactive system design have evolved into various forms, meanings and levels of details. Corresponding to Kuutti (1995), Carroll (2000b) also recognizes the needs to address the wider context of work being supported with the system as well as the detailed interactions. As information systems were becoming the norm in various settings, its context of use was also getting more standardized. Related work in the domain of software engineering, which deals with the more technical aspects to implement the systems, systematize scenario use e.g. by proposing approaches and tools that strive to structure the identification process of "rich" contextual and "narrow" application scenarios. For example, the CREWS project (1999) focused on requirements engineering and delivered approaches to model user goals using scenarios to elicit and validate requirements (including Haumer et al., 1998; Maiden, 1998; Rolland et al., 1998b; Weidenhaupt et al., 1998).

Another example suggests the relation between scenarios with the use case approach from object-oriented software engineering (Jacobson, 1995). Use cases recognize actors, their goals and what they can do with the system, which gives a graspable view of the system's capability and behaviour. Each use case is a complete series of events, described from the point of view of the actor; actors may be end users, other systems, or hardware devices (Jacobson et al., 1991). The use cases may be described in different scope, for instance in abstract level (business use case) or system level (system use case). Use cases aim to make an easier transition from the conceptual abstraction to the programming logics, and therefore strive for a complete coverage of all interaction with the system. Scenarios, on the other hand, are used with a different aim. The aim is not completion, but rather a "just right" coverage to inspire and guide the design process. Scenarios also exist on different levels and can be considered as specific instances contained within use cases.

Another work by Robertson (2004) lays down a model to explain the identification of business requirements from scenarios that are constructed from business use cases. The model advocates the use of scenarios with a business boundary rather than a product boundary to discover business requirements. Identifying business events is important in this technique. A business event is something that happens, within the boundary of the investigation, to which there is a pre-planned business response. Therefore for instance, an identification of a business event "a customer wants to book a taxi" leads to an identification of a business use case "make taxi booking". An informal sketch of the scene is necessary to get a realistic understanding of the use case. Stories from users and stakeholders help to give shape to the sketch of the scene. With enough information, the sketch can be formalized and the scenario steps identified. These scenarios, referred to as requirement scenarios, simulate what happens within the boundaries of a specific scene to discover the business requirements, i.e. the top-level requirements for the system.

From the examples above, scenario-based approaches in software/system design show a consistent effort to systematically break down the abstract, high-level, contextual design data into detailed, precise, logical system requirements. As Information Systems are becoming the norm of various work practices, a large part of its context of use is getting more predictable to the system designers. Therefore it was possible to isolate most of the elements within the system's use situations and to focus on its functionality and logics. Although, a new technology for IS could also prompt for alternative use of scenarios as observed in Bodker (2000). While recognizing the importance of scenarios that generalize users' actions as represented in usability research or software/requirement engineering, Bodker (2000) also proposes scenarios that emphasize critical or even caricatures of situations to help users and designers be creative in design. Examples of plus and minus scenarios illustrate caricature future 
situations about the then new mobile information technology. The extremes highlight the positive and negative implications of such technology for the purpose of reflection. These caricatures become efficient media to harness users' and designers' focus on particular situations, problems and parts of solutions. Therefore, besides providing contexts to explain why the design is the way it is, the plus/minus scenarios can also inspire potential design agendas. Apart from these special cases, the approaches to utilize scenarios in software/system development remain structured and systematical, and aim for a complete coverage of all the possible way to use the product (i.e. a software application).

In the design of tangible consumer products, there is less possibility to systematically address the design situation or to completely cover all possible use cases. There are endless combinations of use elements involved in consumer products, referred to earlier in Chapter 2 as dynamic use situations. The dynamics include among other a variety of users who have different goals and settings while using the product, which correspond to the multiple functions an intelligent consumer product usually offers. Figure 3.2 illustrates how the use aspects of such a product relate with dynamic use situations.

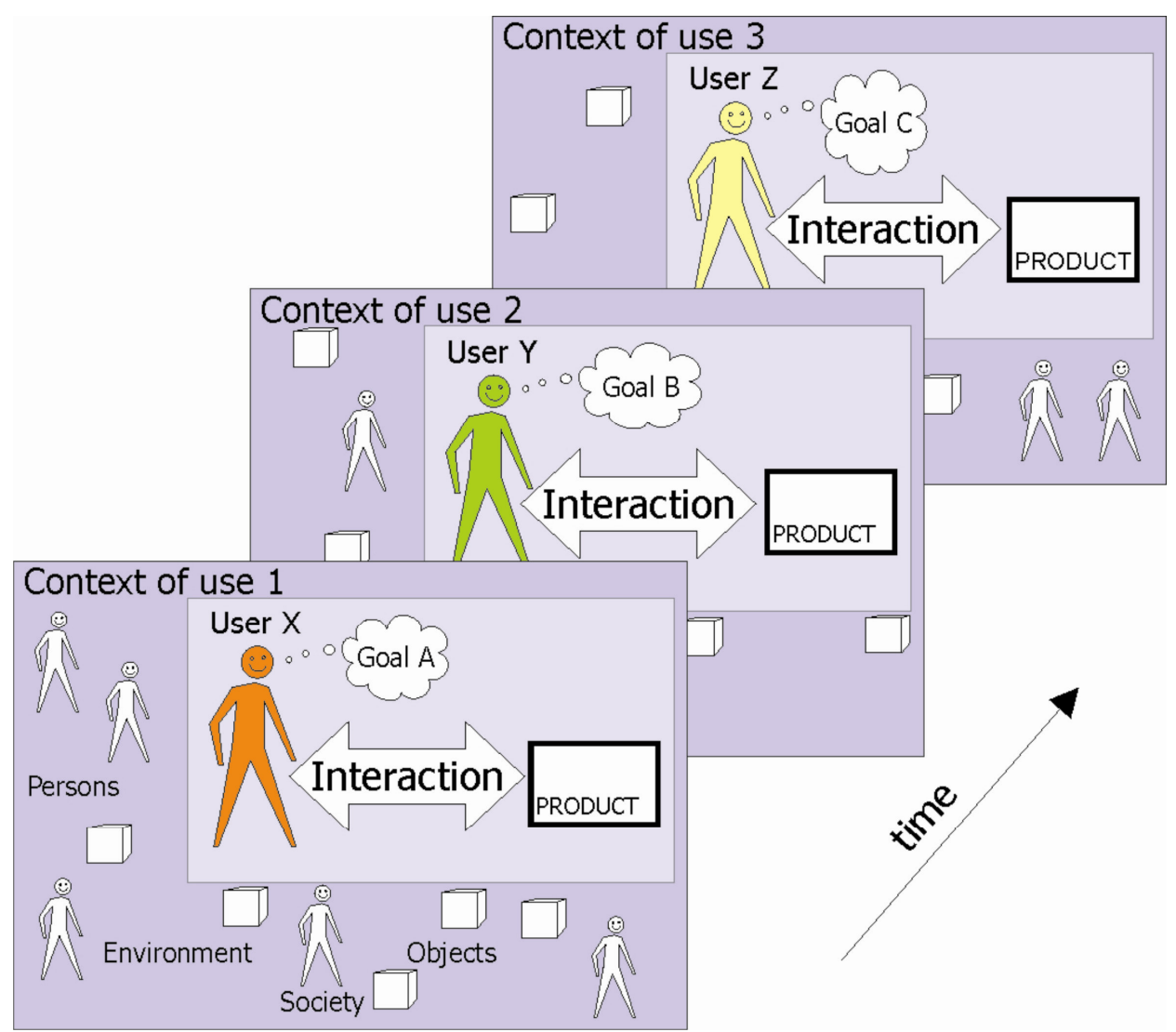

Figure 3.2: The possibility of a multitude of scenario elements in dynamic use situations (Brouwer and van der Voort, 2006).

Instead of aiming to be complete and exhaustive from the start, scenario-based product design focuses on making concrete a set of scenarios that cover the necessary use situations, and later iterates as more information is discovered. The idea is to give a big enough picture for the designers to start working on refining the design problems and solutions. By using scenarios, the designers are also guided to identify the elements of use situations, which include actors, their goals and characteristics, settings, processes (actions and steps) and events. With other influential factors (e.g. project 
type, work culture, or team composition), designers can intuitively use the flexibility of scenarios to accommodate their needs. For instance, the scope, level of details or point of view of scenarios can be adapted to the specific purposes and audience.

Independent from the developments in the Software Engineering and Information System domain, designers of tangible consumer products have proposed design by storytelling, especially to empathize with users. For instance, Moggridge (1993) describes an early scenario-based approach to address design of products for the elderly that includes a four-step process for design: (1) understand the users, (2) observe them, (3) visualize the possible solutions and (4) evaluate them. The first two steps dedicate a great attention towards the target users (i.e. the elderly), taking in information and empathizing with their wants, worries, and physical and mental limitations. Solutions are then visualized through scenario building and storytelling without any initial constraint to the technical and financial implications. The evaluation step involves user evaluation with a full range of the people for whom the designed product or service is intended. Moggridge (1993) claims that storytelling or scenario building is the key to actualize the observation and visualization steps, yet does not assume anything about the usefulness of the stories or scenarios beyond those steps. Another work by Suri and Marsh (2000) studies the development of many consumer products, in which scenario building is shown to be a powerful exploration, prototyping and communication tool. It answers the needs of human factors practitioners who are continuously improving ways to better communicate their contributions, and to involve the users as important stakeholders at all stages of the design process. Both Moggridge (1993) and Suri and Marsh (2000) emphasize scenario building as a useful tool early on in the product design process. However they do not fully address the benefits in the later phases of design process, rather than as an impromptu design tool. Both researches showcase examples of scenario building as an improvised, intuitive, and loosely-defined approach, which is true to the current practice of product design.

Summarizing, up until now the findings in this research indicate that scenario-based approaches are heuristic and cannot be comprehensively generalized across cases or contexts. An overview of scenario uses for a full design process is still missing. While indeed any scenario-based approach needs to be tailor-fit to the particular context, an overview will also help answer the challenges in applying scenario-based design (as introduced in Chapter 2). As this research focuses on the more specific area of scenario creation, use and management, a further literature study is conducted on several topics that relate to the mentioned area. The next section gives the overview of the findings in the literature study, which partially also address the overview of scenario use.

\subsection{Towards Scenario Creation and Use}

The section provides an overview of existing works relevant with our research focus on scenario creation, use and management. These works can be classified into 3 interrelated categories. Firstly, researches that attempt to understand scenario structure by deconstructing a scenario/story to its elements. Secondly, works that address the necessity to recognize and classify the different purposes of using scenarios. Thirdly and lastly, researches that propose methods and tools to support the process of creating, using and organizing scenarios.

\subsubsection{Scenario Elements}

The backbone of any scenario is a narrative. The elements of a scenario, which have been introduced earlier in Chapter 2, resemble those of a narrative. A study of narrative structure in Russian folklores by Propp (1958) has inspired many later researches in scenario modeling. Carroll (2000b) identifies elements of a scenario according to 
Propp's studies. Later work by Rosson and Carroll (2002) elaborates the scenario elements and emphasizes the practical values of knowing what they are. While the actual content of a scenario will depend on its purpose in the particular case, these elements remain consistent. Therefore the product design domain could also use the identified scenario elements as a basis for structuring a scenario. To reintroduce the elements within a scenario that represents a use situation, Figure 3.3 illustrates these elements and their interactions within a scenario.

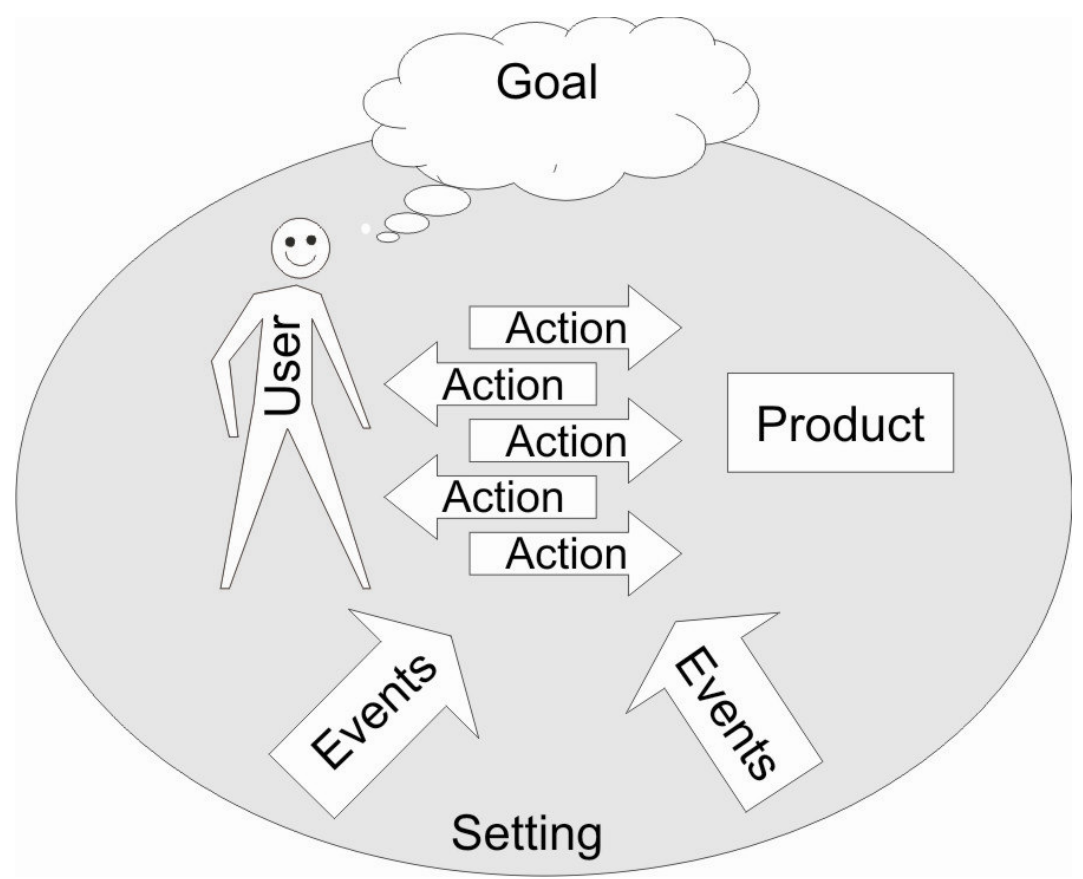

Figure 3.3: A scenario contains at least a user, who has a goal, a product, within a setting; actions and events happen as the user interacts with the product'1.

Also based on Propp's narrative structure, Potts (1995) suggests a scenario schema inspired by story schemata. Similar to stories, scenarios have protagonists with goals, start with background information in place, and most importantly make a point to interest the reader. The scenario schema aims to help designers develop a limited set of salient scenarios. While schemata do not qualify as generative grammars, they can be useful as design heuristics. Hobbs and Potts (1998; 2000) elaborates this work and contributes in a hyperscenario framework to model scenarios and their composite elements. This framework is aimed at supporting decision-making by looking deeper into scenario ontology. A scenario representation is created using the morphology of narratives and the activities of storytelling, and implemented in explicitly marked components referred to as Scenario Markup Language (SCML). The hyperscenario framework formalizes and externalizes the scenarios into a representation accepted in computational language, yet generic enough to extend many disciplines. The hyperscenario framework shows one way to structure the varying elements of a scenario. It inspires this research to identify the relations between elements as a basis for structuring scenarios.

The examples reveal how a logical nesting structure of scenario elements can be created. However, little is explained about the relations between elements as the

\footnotetext{
1 Illustration credited to Mieke van der Bijl-Brouwer.
} 
structure is taken at face-value. Within the context of this research, a rigid structuring of scenarios would not be of added value since the scenarios needs to be flexible and accommodative to whatever purposes they are created for. It is therefore more important to understand the relations and the influences between scenario elements to help practitioners create salient scenarios. To illustrate the complexity of use situations which the scenarios are based on, Figure 3.4 presents the basic parts that compose the scenario elements and how they could be of influence to one another. This relationship diagram of scenario elements has appeared in Anggreeni and van der Voort (2008b).

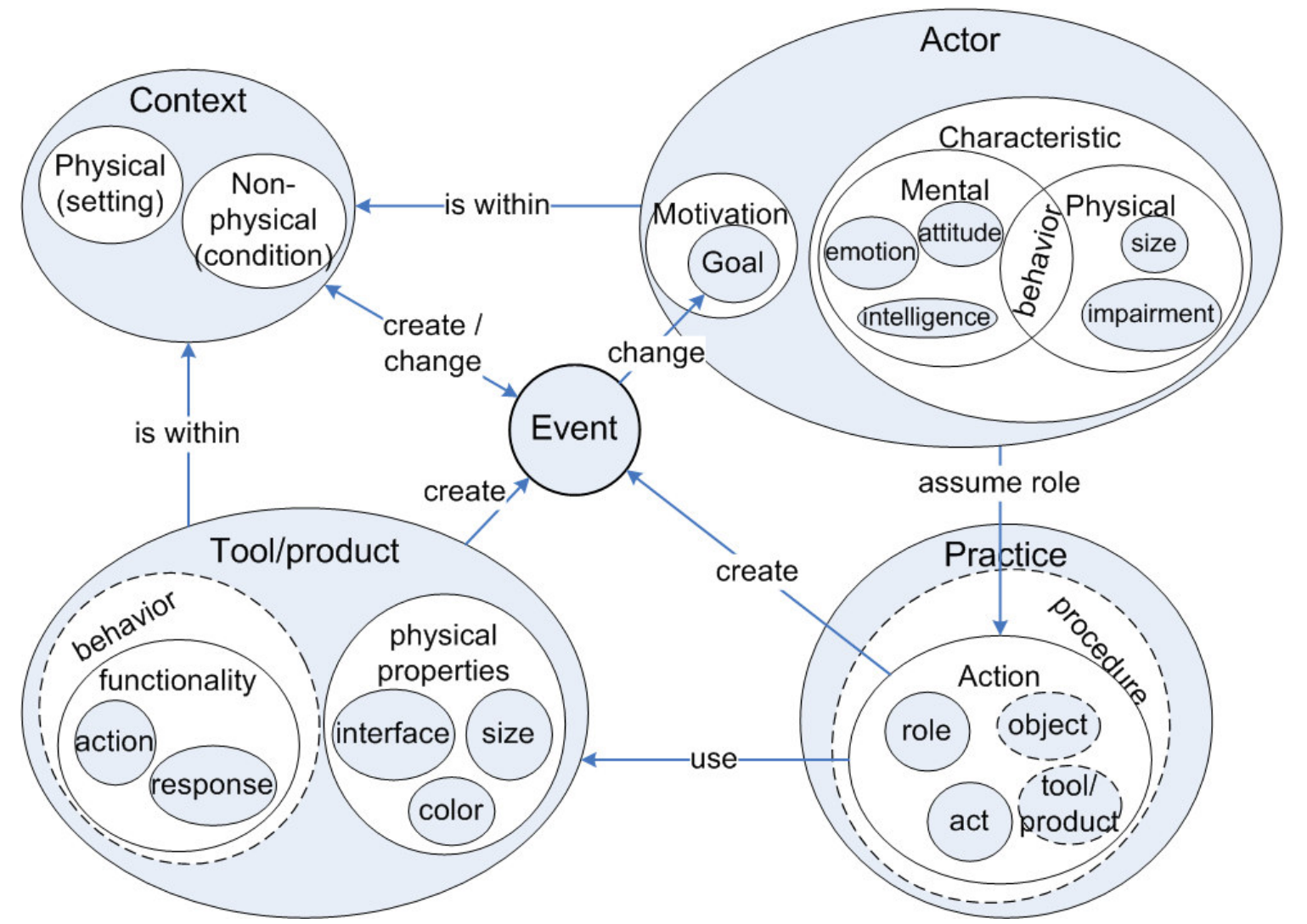

Figure 3.4: Scenario elements, their subcomponents, and influences to one another (Anggreeni and Van der Voort, 2008b).

The mentioned researches have shown examples of the perspectives on elements that make up scenarios and their structuring. An adaptation of the works to the context of this research has been presented in this section. This knowledge of scenario elements is essential as the basis for understanding the creation of a scenario. To complement this knowledge, an understanding of the various scenario uses and their purposes is required to complete the bigger picture. The next section presents an overview of various scenario uses in a design process.

\subsubsection{Scenario Use Classification}

To classify scenarios in a sensible way, it is essential to include the context in which the scenarios are being used. Any of the following factors could provide valuable information about the nature of the scenarios: the phase of design process, the target audience, the supposed setting when showing the scenarios (in a board meeting, a product fair/exhibition, etc), the product characteristics (daily appliances, or machinery to be used by professionals), the structure and working culture of the design team (formal versus informal, allocated man-hour, etc). These factors influence the choice of design activities, and consequently the purposes of using scenarios. 
The variety of purposes stems from the specific design questions that can be answered by using scenarios. For instance, Nielsen (1990) proposes a taxonomy of scenarios by their purposes within heuristic evaluation of User Interface (UI) design. The purposes are to communicate user interface rationales to an audience (i.e. the users or colleagues), to structure thinking, and to test user interface or existing $\mathrm{HCl}$ theories. While this taxonomy is completely logical within the UI design context, very little is explained about the content differences between the types of scenarios. A scenario to communicate user interface rationales, could have structured the designer's thoughts during its making. Later when receiving feedback during user testing, it becomes a tool to test the user interface. The taxonomy does not specify whether the different purposes could actually be served by the same scenario.

Another work by Campbell (1992) puts forward four main purposes of scenarios within the context of Human-Computer Interaction $(\mathrm{HCl})$, which are compatible with Nielsen's taxonomy. The purposes to (1) illustrate the system, to (2) evaluate its functionality, and to (3) design the system's attributes and features, are basically a different formulation of the purposes for communicating and testing the design as well as structuring the thinking process. Campbell (1992) also supports the idea of using a collective set of scenarios to (4) explain and test $\mathrm{HCl}$ theories. Based on collaborative efforts, the scenarios could serve as "test cases" to refine the theories and in turn stop UI designers from building interfaces that cause user errors.

There are endless ways to call scenarios based on their purposes and roles in the design process (a.o. Nardi, 1992; Wright, 1992; Carroll, 1995; Karat, 1995; Carroll, 2000b; Alexander and Maiden, 2004). On an abstracted level, the purposes always relate to either illustrating solutions and their alternatives or identifying potential problems, as recognized by Bodker (2000). The early proposals to classify scenarios are outgrown by the evolving scenario-based approaches. Therefore, rather than labelling scenarios by their purposes, more effort to understand the nature of scenarios in general is needed, as also argued by Wright (1992). These distinguished purposes have explained the motivation of using scenarios, and given a starting point for this research to explore the processes that bring forth the scenarios.

To understand the big picture to which these purposes can be placed, a framework is needed to abstract these different purposes into a knowledge that is useful in this research. Such framework is encountered as a part of a collaborative research project, Cooperative Requirements Engineering with Scenarios (CREWS, 1999). The CREWS project aimed to seek a deeper understanding of scenario diversity, and later used the knowledge to improve methodological and tool support for scenario-based requirements engineering. Rolland et al (1998a) provides the foundation for further research within the CREWS project by developing a scenario classification framework. The framework is based on a comprehensive survey of scenario literature in requirements engineering, human-computer interaction, and other fields. Figure 3.5 illustrates the four facets to classify scenarios they distilled. 


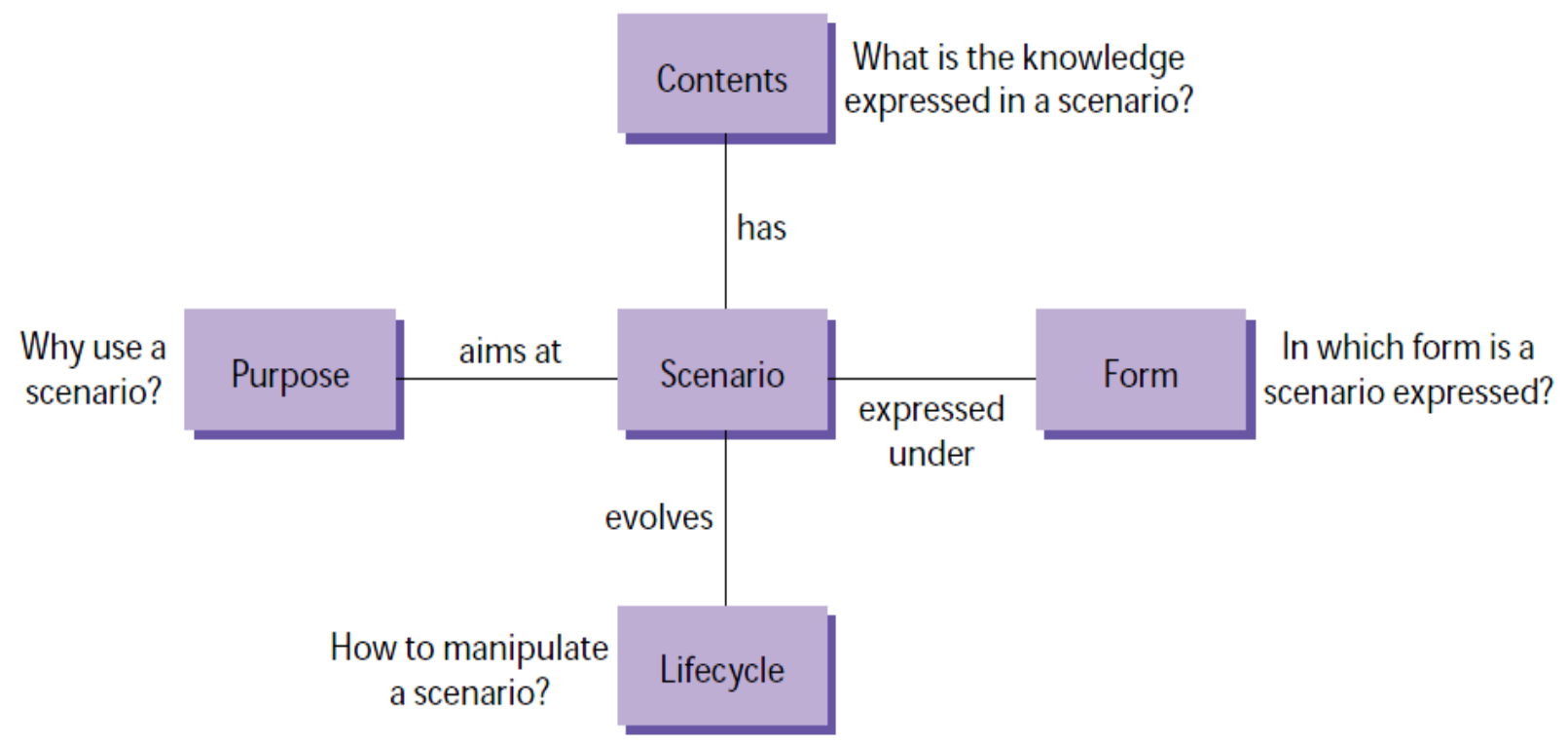

Figure 3.5: Four views on scenarios: form, purpose, content, and life cycle (Rolland et al., 1998a).

Subsequently others within the project are able to use the scenario classification framework as a base for their works (a.o. Jarke et al., 1998; Weidenhaupt et al., 1998); these works will be briefly addressed later in this chapter. However, the CREWS framework is developed to register scenario use exemplars from the software industry with the aims to obtain the state of the art of scenario-based requirements engineering. The already developed scenario-based approaches from this domain (requirements engineering for software development) make it possible to execute the CREWS approach. Scenario-based product design, on the other hand, is in its early stage and currently still develops through adapting scenario-based approaches from other disciplines. On the contrary to the CREWS framework, this research requires a scenario classification framework that can inspire and be built towards scenario creation and use.

Summarizing, existing scenario classifications do not provide solid enough basis for scenario creation and use. Therefore, to combine the findings so far, a scenario use roadmap is developed to establish the understanding on different scenario uses and how they fit in a scenario-based product design process. The next section explains our scenario use roadmap and the scenario types that have been identified. 


\subsubsection{Scenario Use Roadmap}

The proposed scenario use roadmap aims to be a common-ground abstraction of scenario classification, as well as to show the big picture of a design process using scenarios. The design phases used in the roadmap are basically a simplified extraction from various engineering design models (Shigley and Mischke, 1986; Ullman, 1992; Pahl and Beitz, 1996). Although these phases may not always applicable to all design projects, they are generic enough to suffice the discussion. The roadmap as illustrated in Figure 3.6 presents the design phases, each with commonly delivered artefacts and identified potential scenario uses.

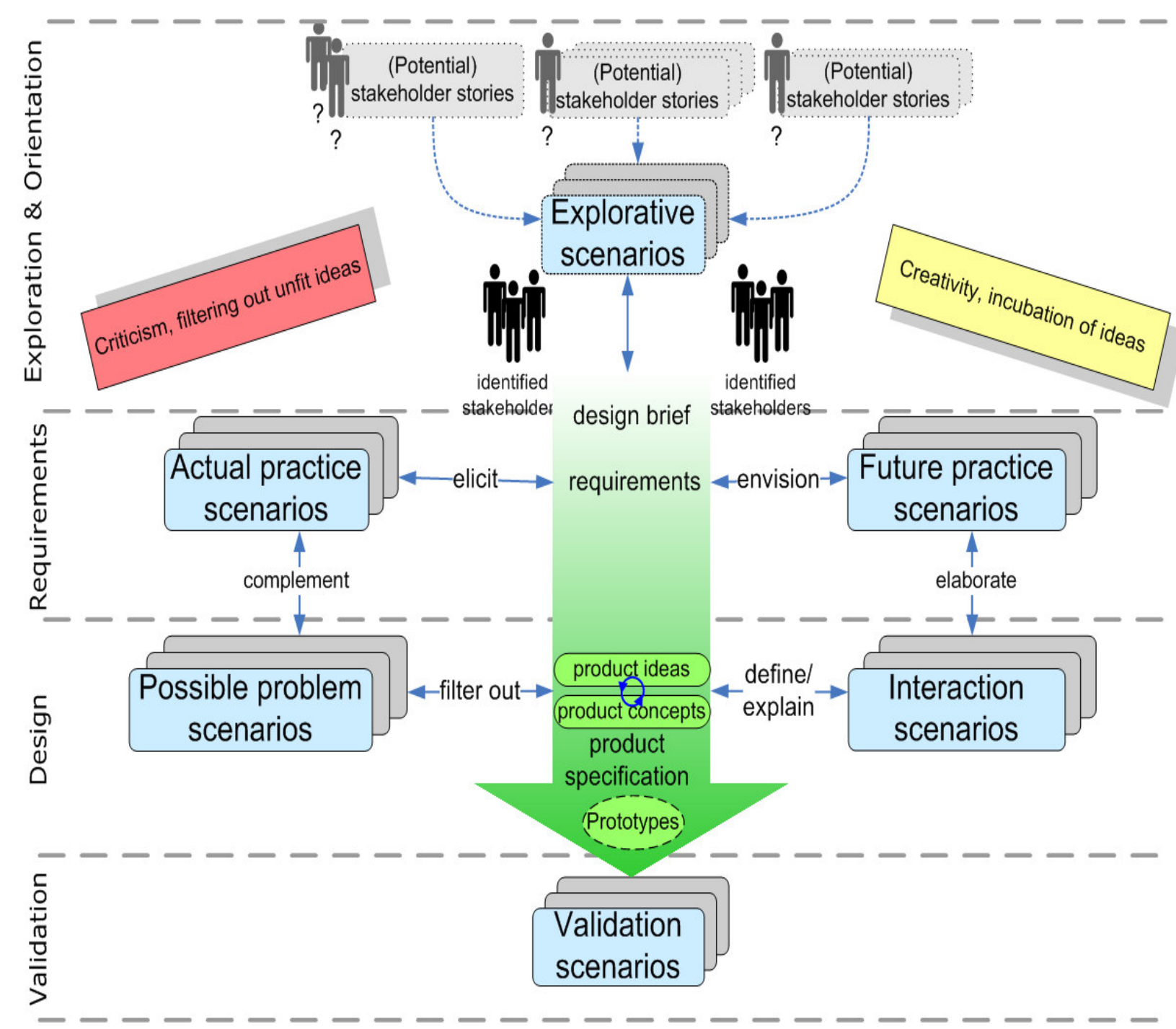

Figure 3.6: A roadmap for possible scenario use in a design process, showing how scenarios are related to one another and to other in-between design results (Anggreeni and Van der Voort, 2008b).

The following subsections explain what is assumed to be happening in the design phases from a theoretical perspective. This theoretical overview of various scenario uses has been presented in Anggreeni and van der Voort (2008a; 2008b). Chapter 4 will discuss how these assumptions are verified with design practice. For now, they serve to provide a common ground to discuss the types of scenarios that can be useful in each different phase. 


\section{Phase 1. Exploration and Orientation}

Design problems in any design project are often ill-defined. However, despite the uncertainties in the phase, the design team must dare to create tentative solutions and understand the problem boundaries. To make sound decisions in this early phase amid the uncertainties, the design team members inform themselves well on the subjects relevant to the design problem. Stories from potential stakeholders often reveal important aspects of their professional or personal lives, parts of which will be affected by the design. Adopting scenario planning for decision making (Schwartz, 1996), the knowledge from stakeholders and other sources could be synthesized into a small number of focused explorative scenarios. Explorative scenarios describe some versions of the future which are all equally plausible; yet they also accentuate the most important and uncertain elements within a world of certain and predictable elements. As a result, the design team gains awareness of the possible consequences of their design in the plausible futures. For example, designing a bicycle for a market of 5-10 years in the future requires designers' understandings of the plausible situations in which people use bicycles. How is commuting like within 5 to 10 years? Could it be that the use of bicycles is triggered due to government imposing environment policies? Is there any new traffic regulation or infrastructure to be implemented within 5 to 10 years? The answers could lead to a broad range of design directions where exploration is necessary.

\section{Scenario Types}

- (Potential) stakeholder stories serve as an initial study into the domain where the product is going to be used. These stories reveal what matters to the (potential) stakeholders, which aspects of life or work they want to improve, etc. Interviews, surveys and ethnography are among the techniques than can be used as input.

- Explorative scenarios help the design team to reflect on their design strategy, creating awareness of the threats and opportunities along their decisions. Creating sound explorative scenarios requires knowledge that can be informed by literature studies, survey results and experts in the related domain. In practice, designers might not be actively involved in the creation of these scenarios. When the design project is still being defined, other members of the design team (e.g. managers or marketing) have a more active role to create a business case together with the stakeholders. The explorative scenarios could improve their communications in the process.

\section{Phase 2. Requirements Capture}

A set of requirements is an elaboration of problem and solution definitions. In practice, problems and solutions evolve together throughout the design process: every proposed solution affects the problems, whereas problem redefinition reveals possible solutions that lie outside the boundaries of what was assumed to be possible (Cross, 1994). Therefore, one approach could begin from the actual situation to identify requirements. The actual practice scenarios capture the current (problematic) situations and based on them, draw requirements. Another approach could start from solution ideas that, by designers' intuition, might work. The designers could then envision more requirements from the imagined future practice. Future practice scenarios describe imagined futures with the solution ideas: 'would people use the product?', 'how might people perform their activities if such product were available?', 'how would the product affect their life?'. Both approaches propel each other into a more detailed set of requirements. 


\section{Scenario Types}

- Actual practice scenarios describe users' problems, dissatisfactions, needs and wishes in their current practice in a concrete manner. The concreteness is achieved by completing each scenario with all relevant information, so that there is no room for misinterpretation. Interviews, ethnography and contextual inquiry with users (and possibly other stakeholders) inform the creation of these scenarios.

- Future practice scenarios exploit designers' intuition, but do not completely rely on it. These scenarios are projections of how the early product ideas would change the use practice, and could be accompanied with mock-ups or demonstrators. By keeping a firm connection with the empirical requirements, the ideas are directed to fulfill the user needs. By using future scenarios instead of prematurely building prototypes, there is only minimum risk in case the ideas turn out to be unsuitable. The stakeholders can be more actively involved in the shaping of the solutions because the future scenarios can be easily molded with their views on the futures.

\section{Phase 3. Design (conceptual and detailed)}

The design phase requires both creativity and criticism to mingle. Developing product concepts is a cycle on its own: the design team thinks of a product concept using information from stakeholders, evaluates it in different use situations and makes modifications to the concept. In this phase, the designers produce many sketches depicting their ideas to bring on the solutions. The interaction scenarios could regulate this activity. They are detailed accounts of the future practice scenarios, thus formulating answers to the question "How does the user interact with the product to make future practice scenarios happen?". Therefore, the interaction scenarios are actually an inseparable part to the sketches (i.e. product ideas/concepts), though sometimes they are not made explicit. The interaction scenarios could belong to different levels of interactions. In the beginning when the designers are still refining the product concept, the interaction scenarios can represent it as a 'black-box' product with a set of behaviours. Having concrete scenarios to work with, it is easier for the designers to fit a concept into different use situations iteratively, making sense of the proposed solution in the real life. Inviting users' opinions, for example in a 'scenario walkthrough' session, could assist in determining the concept details. The interaction scenarios could then be refined by adding more details to the interactions and interfaces.

Possible problem scenarios expose weaknesses of the product concept, especially in extreme and critical situations. The initial requirements capture might not discover all extreme and critical situations, which could be remediated by a close and continuous contact with users and stakeholders. Possible problem scenarios could also describe unanticipated problems that may rise due to the proposed solutions, thus requiring criticism which is less biased when it comes from stakeholders. In conclusion, both scenario types are more effective when created, discussed and criticized together with the stakeholders.

\section{Scenario Types}

- Interaction scenarios describe the interactions between users and the product concept to actualize the futures as claimed in future practice scenarios. The interaction scenarios undergo transformation from abstract to detailed, and eventually function as rationales to the resulting design specification. The opinions of users are most valued, and therefore their involvement through participatory design is important (e.g. Muller and Kuhn, 1993; Svanaes and Seland, 2004). 
- Possible problem scenarios describe problematic events or situations against which the product concept should be tested. These events or situations could be left unnoticed during the requirements capture using interview and ethnography (observation). Complementally, designers and stakeholders could conduct a brainstorm session to identify critical, extreme or dangerous events or situations that could happen during product usage. Probing technique (Wensveen, 1999) could also be used to invite users to share their personal experiences which they think relevant to the product being designed.

\section{Phase 4. Validation or Pre-fabrication}

The design phase aims to generate solutions which fulfil the design requirements with as minimum compromise as possible. To avoid overlooking any requirement ("I really did think of that, but I forgot...') or more subtle criteria ('Did the user say he likes it this or that way?'), the design team needs to evaluate the solution or solutions by means of validation scenarios. Validation scenarios take inspiration from all other previous scenarios. The validation process itself could cover several design aspects (e.g. functionality, usability, safety and branding profile) or specific product parts (e.g. lock mechanism, steering mechanism or motor). For each of these design aspects or specific product parts, validation scenarios can be composed by combining scenario elements of existing scenarios to give a good coverage of all possible use situations (see Figure 3.3 for the overview of scenario elements).

\section{Scenario Types}

- Validation scenarios, especially the ones intended for usability testing must be complete, i.e. comprise a complete set of scenario elements. As defined in ISO 924111 (ISO, 1998), the usability of a product applies to specified users (element: actors) with specified goals (actor's sub-element: goals) in a specified context of use (element: context). This phase must detect any remaining deficiency before the product design is manufactured and marketed. Therefore, a rigorous participation from all stakeholders within the validation phase is requisite. Any change to the product specification should be only to streamline or fine-tune it.

This section has summarized the various aspects of scenario uses by means of a scenario use roadmap. The roadmap is an abstraction of a scenario-based product design process, which shows the overview of scenario types within the process. The roadmap as the big picture still needs to be complemented with the knowledge on scenario creation, use and management in a design process. A study on existing models, methods and tools for the mentioned purpose has been conducted and the results are presented in the next section.

\subsubsection{Scenario Construction}

Some works have proposed to explain the creation process of scenarios, which is generally referred to as scenario construction (e.g. Antón and Potts, 1998; Rolland et al., 1998b; Leite et al., 2000). Based on the types of contribution, three main clusters can be recognized: heuristic, process model (formalization), and tool support. While they complement one another, the fundamental part of any scenario construction approach is the heuristic. A model is a framework that formalizes the set of general knowledge and common sense rules that we call heuristics. A support tool is always based on specific heuristics and formalized models. For brevity, this section only addresses the approaches that are most relevant and inspiring to this research. 


\section{Heuristics}

Online Cambridge Dictionary (2010) defines 'heuristic' as "(of a method of teaching) allowing students to learn by discovering things themselves and learning from their own experiences rather than by telling them things". The term 'heuristic' in this research context refers to a set of generic steps which explains the underlying concept of a scenario-based design process. Design methods are the best representative for heuristics. By nature, a heuristic does not set certain values or constraints, and can be interpreted into varied actions. This flexibility often means that its success largely depends on the skill and experience of its implementers. Evaluation on a heuristic approach is qualitative, and the productivity of a specific strategy developed according to the heuristic can be assessed only after it is done. A large body of research on scenario-based approaches from various domains reflects this.

Carroll (1995) binds together various views on how scenarios help to envision the use of the then new information technology in work settings. This work mainly shows exemplars of scenario uses in different cases, and does not propose a unified scenario-based approach. Carroll (2000b) refines scenario-based design into a well-rounded approach that addresses the realistic aspects of design (i.e. Information System design). Realizing that scenario descriptions are fundamentally heuristic and cannot be complete, an eclectic approach to make sure the scenarios have good coverage and minimal bias is suggested. Carroll (2000b) proposes seven heuristic methods, which are largely complementary, as a fairly comprehensive methodology to generate scenarios. In brief, scenarios can be derived from the involvement of target users through ethnographic field study and participatory design, the existing knowledge about a specific domain through reuse of prior analyses, scenario typologies and existing theories, the possibility of new technology by creating technology-based scenarios, and lastly, inspired by DeBono (1990)'s brainstorming technique, transformations. While most of these seven methods are generic enough and should be on the process level, the last method 'transformations' is specific on the actual identification and creation of scenarios.

The transformation method aims to broaden the set of empirical scenarios. Based on brainstorming techniques, transformation follows a simple set of manipulation. Firstly, by challenging each action in each scenario with "What could go wrong?". Whether it is a scenario describing current or future practice, this first technique expects to reveal problems and needs that are initially not discovered. Secondly, by exchanging points of view and ask how other actors would regard each scenario. A product interacts not only with a single actor. Taking up other actors' points of view would ensure a better coverage of the product uses. Thirdly, by exchanging tools and other task objects to envision new scenarios. This technique acts as a bridge from observed scenarios to the envisioned scenarios. Fourthly, a rather brute-force technique, is by creating a Cartesian product 2 of scenario elements such as actors, goals, actions. The last technique requires the design team to use their own judgement to make sure that the most significant combinations of elements are addressed in scenarios.

To summarize the discussion, heuristics are basically high-level, abstract and aim to inspire rather than prescribe. A designer attempting to create scenarios could learn from the mentioned works independent from which domain they have been developed. While the heuristics allow flexibility, they do not provide the specifics and therefore its success largely depends on the skill and experience of the people using it. In the case of a design project, this means that designers have to rely on their intuition to determine an appropriate way to use the methods. Other works in this research area

2 The set of elements common to two or more sets. As an example $\{1,2\} \times\{3,4\}=\{(1,3),(1,4),(2,3),(2,4)\}$ 
however, have actively contributed (formal) models in an attempt to guide scenario construction in a more structured way.

\section{Models and Tools}

Various models have been proposed to explain the composition, construction process and management of scenarios within specific design phases. The cumulative knowledge and experiences of these heuristics allow the construction of more prescriptive models and frameworks. Since these models and frameworks are often based on knowledge and experiences in particular cases or domains, consequently they are not always relevant with this research. Nevertheless, these existing works inspire our research by elaborating how their approaches address specific challenges in design processes using scenarios. This research therefore expects to learn from the approaches and to identify how parts of them could be adapted to the product design domain.

CREWS-L'ecritoire (Rolland and Achour, 1997; Rolland et al., 1998b) highlights the derivation of scenarios from goal modelling and the guidelines for writing and editing quality scenarios. With its application area in information system design, the approach strives for a systematic use of scenarios to capture system requirements. It does not include scenarios focusing on usability issues within its scope. To identify requirements and scenarios of multiple levels of details, CREWS-L'ecritoire suggests a systematic loop of (1) starting with a (user) goal, (2) describing a scenario as a possible concretisation of the goal, and (3) analysing the scenario which leads to finding out more goals. Within this approach, a requirement consists of a pair of goal and scenario (see Figure 3.7). Therefore, requirements elicitation is also goals discovery. The resulting requirements are represented as use cases, which describe the transactions involving the required system and its environment.

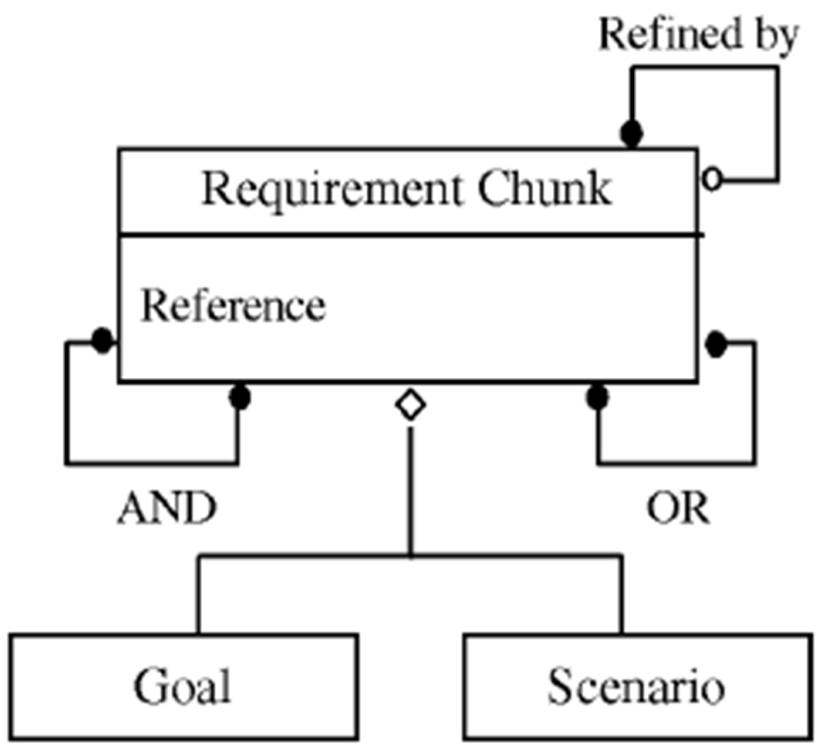

Figure 3.7: CREWS-L'ecritoire relationship model of requirement, goal and scenario.

The defined relation between requirement, goal and scenario is a useful and relevant contribution to any domain which uses scenarios. Furthermore, this research could learn from the structured mechanism on how to identify goals and scenarios, and how to break them down further into concrete requirements. However, due to its specific application area in IS design, CREWS-L'ecritoire leaves out scenario uses that are 
essential for this research, i.e. scenarios that address the dynamic use situations of the product.

A scenario construction process by Leite, Hadad, Doorn and Kaplan (2000) was initiated with a survey on scenario construction processes commonly used within software engineering. The result, a conceptual model referred to as a middle-out approach is proposed to describe scenarios and guide the process. The middle-out approach starts from building a Language Extended Lexicon (LEL) from the application domain (also called Universe of Discourse, UofD). Building the LEL symbols consists of identifying actors and their behavioural responses. Candidate scenarios are derived from these behavioural responses. The candidate scenarios then undergo several stages to finally emerge as scenarios, organized within the more high-level integration scenarios. The approach guides the principal activities in scenario construction process, namely derive, describe, organize, verify and validate. Figure 3.8 illustrates the relation between these activities.

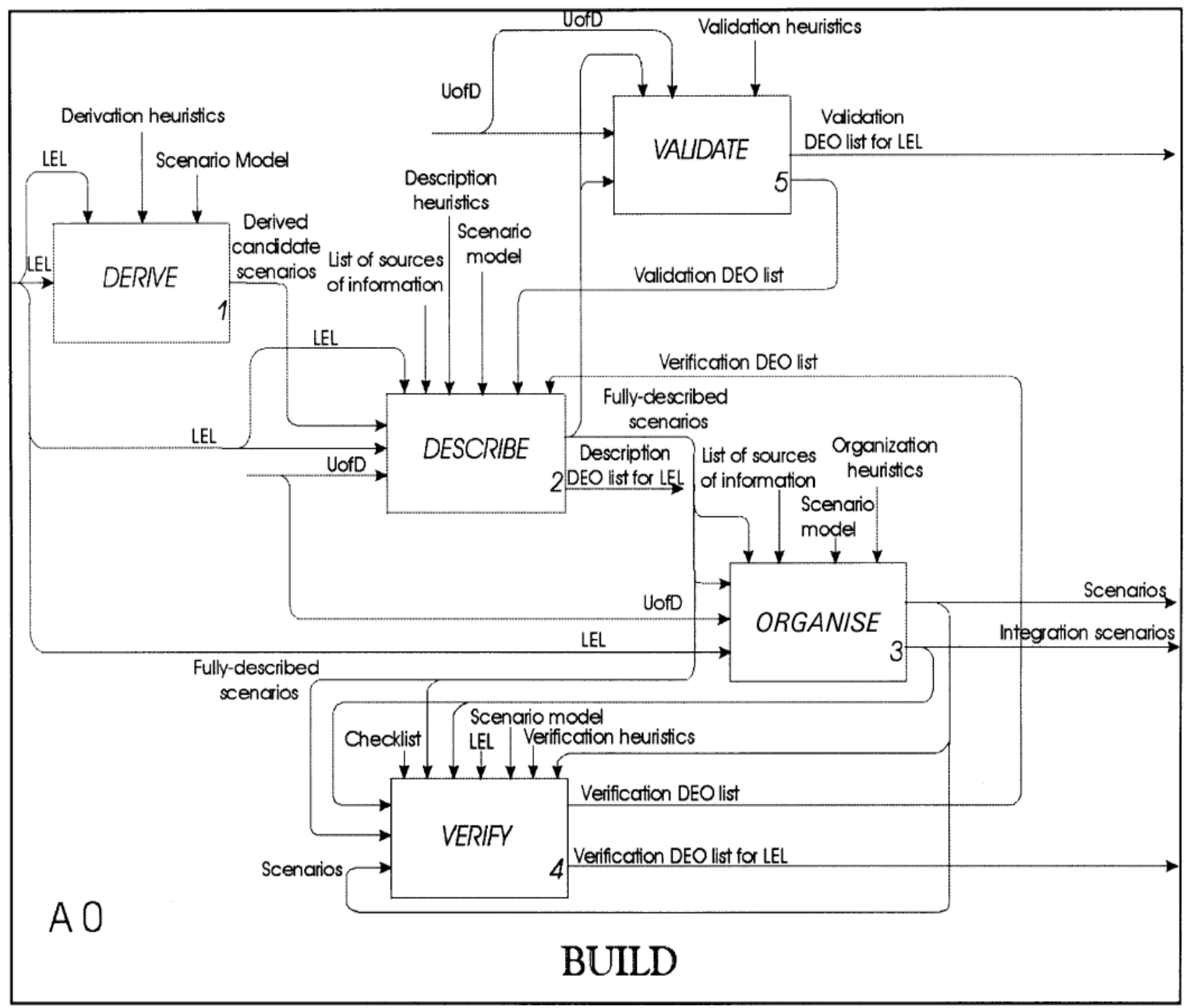

Figure 3.8: The scenario building process represented in $\mathrm{SADT}^{3}$ model.

The middle-out approach explains the scenario building process into a formalized model, which informs this research an effective process of creating, organizing and validating scenarios in software engineering. The lexicon (LEL) also inspires a semistructured way to represent scenarios. However, similar to CREWS-L'ecritoire, the

${ }^{3}$ Notation of SADT: boxes represent activities, left arrows represent input required by the activity, down arrows represent controls, up arrows represent mechanisms and right arrows represents output from the activity. 
middle-out approach does not guide the construction of scenarios required in this research, i.e. ones that take into account the dynamic use situations of the product. The lexicon building is limited to a designated domain, assuming a narrow set of actors and predictable behavioural responses, which is not the case in this research.

Scenario Description Markup Language (SDML) (Penna et al., 2003; Penna et al., 2006) focuses on structuring scenario representation for a system design, as an alternative to guiding scenario construction. Challenged by a variety of semi-formal scenario representations (such as tables, structured text, interaction diagram), SDML chooses combining natural language and formalism to tackle the inconsistency, ambiguity and redundancy common to the semi-formal methods. Just to give an idea how SDML is structured, Figure 3.9 shows parts of the structure. The formalism allows scenario reuse in other phases as well as automatic generation of test cases and state diagrams. SDML strives for correctness (that the behaviour of the system exists to satisfy requirements) and completeness (that all the behaviours required by the users are addressed).

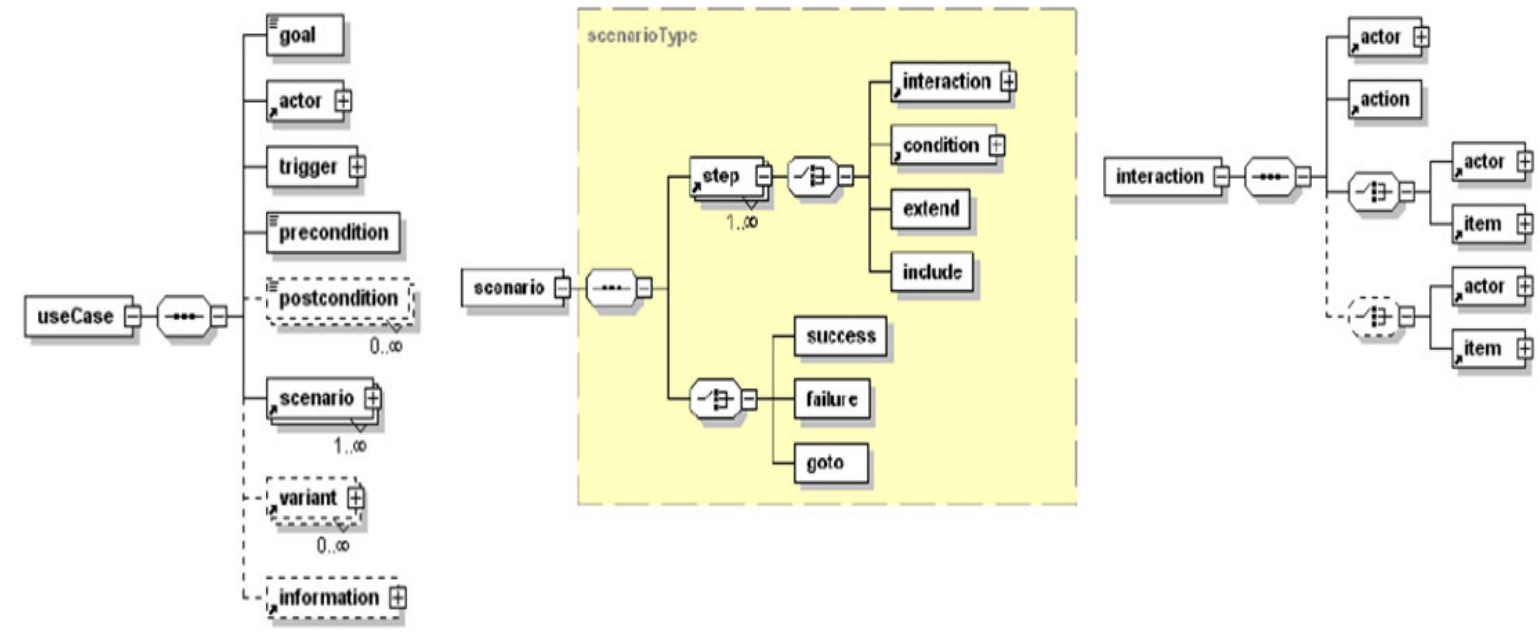

Figure 3.9: The use case, scenario and interaction structure of Scenario Description Markup Language

(SDML).

SDML makes explicit the relationship of scenarios with other necessary data within a system design. Consequently, similar with the previously mentioned works, the SDML structure is not adequate for the more complex product design domain. Furthermore, this approach relies on the assumption that a complete set of system requirements are or can be discovered readily, with a final aim for requirements validation. This might be possible when the scope is limited to only the system behaviour. This research however deals with broader aspects and therefore does not gain much from SDML.

Design Information Framework (DIF) is a structured approach to address narrative scenario generation for product design (Lim and Sato, 2006). As it has been developed for the specific product design domain, DIF accommodates the multiple aspects of use situations by explicitly including them as "aspect models". It contains three basic steps: (1) organizing and structuring user research data, (2) creating multiple aspect models and integrating them into integrated models as necessary, and eventually (2) generating scenarios by translating the aspect models into a narrative format. Figure 3.10 gives an overview of the steps to generate scenarios. 


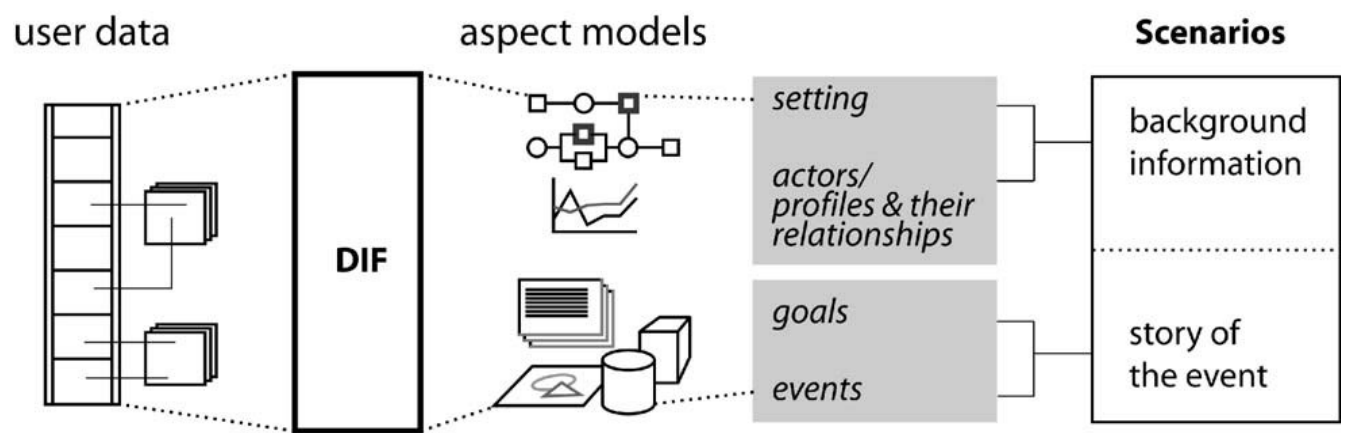

Figure 3.10: The process of generating scenarios with DIF.

The aspect models give a holistic view on the use situations to inform coherent scenario building. DIF has contributed a valuable framework on how scenarios construction should be in product design domain: taking into account not only the product functionality, but also the all-interrelated aspects of use situations. However, the creation of DIF's aspect models demands intensive user research, which is exhaustive and not always feasible in practice.

These examples sufficiently represent the existing approaches that aim to support scenario creation, use and management. Next to these examples, additional works have been studied. However, they share similar characteristics with the discussed examples, in that they lean towards prescriptive and systematic steps, focus the application on specific areas/activities (i.e. mainly requirements engineering), and often do not address the dynamic use situations of the product. As an example, CREWS-SAVRE approach (Maiden, 1998) provides systematic and prescriptive guidance for scenario-based requirements acquisition and validation, yet its application is limited only to reusing existing knowledge in NATURE's object system models (Jarke et al., 1993; In: Maiden, 1998). Another example is the scenario advisor tool (Shin et al., 2005) that provides help in generating a sufficient set of scenarios for the design of socio-technical systems. Its specific focus on socio-technical aspects is rather narrow and idealistic for a practical application. While DIF (Lim and Sato, 2006) addresses the multiple aspects in product design and comes close to the targeted area of this research, its prescriptiveness is very demanding and not realistic in practice.

While it is not possible to address all the works that have been done, the approaches discussed in this section are representative of the current state of this research field. To provide a more accessible summary of the mentioned approaches, Table 3.1 lists their key characteristics. The table structure is adapted from the CREWS classification framework (Rolland et al., 1998a), and takes inspiration from its use in a survey on industrial practice (Weidenhaupt et al., 1998). 
Table 3.1: A summary of the discussed approaches for scenarios creation (construction) and management.

\begin{tabular}{|c|c|c|c|c|c|}
\hline \multicolumn{2}{|c|}{ Scenario Construction Approach } & \multirow{2}{*}{$\begin{array}{l}\text { CREWS L'ecritoire (Rolland and } \\
\text { Achour, 1997; Rolland et al., } \\
\text { 1998b) } \\
\begin{array}{l}\text { Writing and editing guidelines to } \\
\text { scenario authors (heuristic) }\end{array}\end{array}$} & \multirow{2}{*}{$\begin{array}{l}\text { Scenario construction process (Leite et } \\
\text { al., 2000) } \\
\text { Middle-out strategy (heuristic) }\end{array}$} & \multirow{2}{*}{$\begin{array}{l}\text { Scenario Model Environment \&Scenario } \\
\text { Description ML (Penna et al., 2003; } \\
\text { 2006) } \\
\begin{array}{l}\text { SDML methodology (structured \& } \\
\text { heuristic) }\end{array}\end{array}$} & \multirow{2}{*}{$\begin{array}{l}\begin{array}{l}\text { Design Information Framework (Lim } \\
\text { and Sato, 2006) }\end{array} \\
\begin{array}{l}\text { Heuristic \& semi-structured, using a } \\
\text { framework of aspect models }\end{array}\end{array}$} \\
\hline \multirow{5}{*}{ 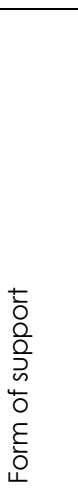 } & Process guidance (1) & & & & \\
\hline & New practice & & & & \\
\hline & Software tool & $\begin{array}{l}\text { Automation of the guiding rules } \\
\text { (instructive) }\end{array}$ & None & $\begin{array}{l}\text { Scenario model manager (design \& } \\
\text { representation), test model manager, } \\
\text { behaviour model manager. }\end{array}$ & [Future work] \\
\hline & $\begin{array}{lrr}\text { Template } & \text { for } & \text { writing } \\
\text { scenarios } & \text { (incl. } \\
\text { grammar) } & \end{array}$ & $\begin{array}{l}\text { Yes, an integrated part of the } \\
\text { software }\end{array}$ & Yes, following Scenario \& LEL models & $\begin{array}{l}\text { Yes, integrated to the scenario model } \\
\text { design }\end{array}$ & Multiple Aspect Models \\
\hline & Glossary (lexicon) & Defined by scenario author & $\begin{array}{lll}\text { Language } & \text { Extended Lexicon (LEL), } \\
\text { Universe } & \text { of Discourse } & \text { (UofD) } \\
\text { vocabulary } & & \end{array}$ & Defined by scenario author & - \\
\hline \multirow{3}{*}{ 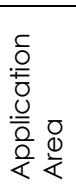 } & Application area & Requirements capture & Requirements capture & Requirements validation & Requirements capture \\
\hline & Optimal domain & System development & $\begin{array}{l}\text { Systems with strong organizational } \\
\text { context }\end{array}$ & System development & $\begin{array}{l}\text { Procedural \& multiple-aspect } \\
\text { domain }\end{array}$ \\
\hline & Generic & Yes & Depends on the overage of UofD & Yes & Yes \\
\hline \multirow{12}{*}{ 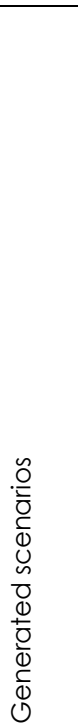 } & Medium & Unambiguous sequential text & Form-oriented written text & $\begin{array}{l}\text { Control flows in nested forms } \\
\text { Blocks for scenario composites }\end{array}$ & Aspect Models, textual narrative \\
\hline & Manner (2) & Semi-formal & Semi-formal & Formal & Semi-formal \\
\hline & Abstraction (3) & Type/abstract & Type/abstract & Type/abstract & Instance/concrete \\
\hline & Level (of granularity) & & & & \\
\hline & Contextual & Yes & Yes & Yes (preconditions) & Yes \\
\hline & (System) interaction & Yes & Depends on scenario author & Yes & Yes \\
\hline & (System) internal & Yes & No & Yes (system validation) & No \\
\hline & Coverage & & & & \\
\hline & Physical context & Resource & Depends on scenario author & - & \begin{tabular}{|l|} 
Spatial layout \\
\end{tabular} \\
\hline & $\begin{array}{l}\text { Non-physical } \\
\text { context }\end{array}$ & Goal & Goal, Responsibility, Precondition & Pre- \& post-conditions & $\begin{array}{l}\text { Activity-role, hierarchical task, } \\
\text { information flow }\end{array}$ \\
\hline & Actor & Goal & Behaviour (LEL) & - & Role-activity \& user profile \\
\hline & Product & Functionality & Behaviour (LEL) & Behaviour, functionality & Functions \\
\hline
\end{tabular}




\begin{tabular}{|c|c|c|c|c|c|}
\hline & Interaction & Information exchange & Information & Information exchange & Action (with/without tool) \\
\hline & Purpose & Descriptive & Descriptive & Descriptive & Descriptive, explorative \\
\hline & $\begin{array}{l}\text { Start-up source (input) } \\
\text { (4) }\end{array}$ & Scenario/Goal & LEL's Behavioural Response & User requirements & $\begin{array}{l}\text { User studies, translated into aspect } \\
\text { models }\end{array}$ \\
\hline & Type (5) & $\begin{array}{l}\text { Actual practice scenarios } \\
\text { Future practice scenarios } \\
\text { (Interaction scenarios) }\end{array}$ & $\begin{array}{l}\text { Actual practice scenarios } \\
\text { Future practice scenarios }\end{array}$ & $\begin{array}{l}\text { Future practice scenarios } \\
\text { (Interaction scenarios) }\end{array}$ & $\begin{array}{l}\text { Actual practice scenarios } \\
\text { Possible problem scenarios }\end{array}$ \\
\hline \multirow[b]{4}{*}{ 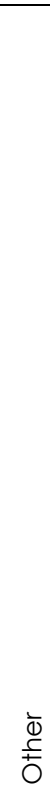 } & Distinguishing feature & $\begin{array}{l}\text { Goal-scenario coupling as a } \\
\text { requirement }\end{array}$ & $\begin{array}{l}\text { Middle-out strategy based on } \\
\text { empirical studies }\end{array}$ & $\begin{array}{l}\text { Formalism, test case generation, } \\
\text { reusability }\end{array}$ & $\begin{array}{l}\text { Multiple aspect models to represent } \\
\text { design information }\end{array}$ \\
\hline & Strength & $\begin{array}{l}\text { Multiple levels of granularity of } \\
\text { scenarios } \\
\text { Structured } \\
\text { identification } \\
\text { Mapping system features to } \\
\text { goals (traceability) }\end{array}$ & $\begin{array}{l}\text { Use of LEL is both flexible and } \\
\text { systematic } \\
\text { Actors' behavioural responses as } \\
\text { scenario candidates is sensible } \\
\text { Scenario management }\end{array}$ & $\begin{array}{l}\text { Addressing the user-system interactions } \\
\text { Well-documented exceptions, } \\
\text { immediately accessible }\end{array}$ & $\begin{array}{l}\text { Multiple aspect models creating a } \\
\text { holistic problem viewing } \\
\text { Aspect models give comprehensive } \\
\text { scenario elements and their links to } \\
\text { one another for scenario } \\
\text { generation }\end{array}$ \\
\hline & Weakness & $\begin{array}{l}\text { Specific to IS design } \\
\text { Deliberate exclusion of the } \\
\text { particular aspects of use } \\
\text { (because they are less relevant } \\
\text { in IS design) } \\
\text { Limited scenario use }\end{array}$ & $\begin{array}{l}\text { LEL as the starting point of this } \\
\text { approach needs to be built first } \\
\text { The quality of LEL depends on the } \\
\text { author's experience } \\
\text { The middle-out strategy is not distinct } \\
\text { on its own; it's a combination of } \\
\text { bottom-up and top-down }\end{array}$ & $\begin{array}{l}\text { Narrow focus on software design } \\
\text { Due to its narrow focus, this approach } \\
\text { assumes that complete requirements } \\
\text { are or can be discovered } \\
\text { Limited scenario use }\end{array}$ & $\begin{array}{l}\text { Relies heavily on user observation } \\
\text { (exhaustive) } \\
\text { Generation of the aspect models is } \\
\text { exhaustive (unless automated) } \\
\text { Selection \& prioritization of aspect } \\
\text { models is unguided } \\
\text { The synthesis and evaluation } \\
\text { activities are unaddressed }\end{array}$ \\
\hline & Case study & ATM & Meeting Scheduler & $\begin{array}{l}\text { ATM, Italgiureweb (web application } \\
\text { development), several other case } \\
\text { studies }\end{array}$ & Phlebotomist in blood sampling \\
\hline
\end{tabular}

(2) The formality of the approach:

- Formal (e.g. follows a modelling language or notation such as use case or state chart)

- Semi-Formal (e.g. scenarios are created as narratives and must use a set of glossary/lexicon)

- Informal (e.g. when the approach only gives the content guideline and allows scenarios to be free flowing narratives as written by the designer)

(3) Whether the created scenarios are on the level of instance (concrete) or type (abstract)

(4) The 'capture operation' in the operation facet of the CREWS framework (Rolland et al., 1998a), i.e. the initial data that is used to construct scenarios

(5) The scenario types according to our scenario use roadmap (Figure 3.6) 
Table 3.1 highlights the distinct characteristics, strengths and weaknesses of the representative approaches. From knowing the essence of the approaches, a qualitative assessment can be performed on what they are good at from which this research can learn, as well as what they are missing that this research still needs to address for the product design domain. Overall, none of the approaches can be a foundation for the next steps in this research, which aims to include other aspects in design activities and not only focusing on the process (as concluded in Chapter 2). Nevertheless, the existing approaches reveal partial solutions that are potentially adaptable to this research, as will be summarized in the next section. 


\subsection{Lessons Learnt for Supporting Scenario Creation and Use}

The existing works as discussed in the previous section have revealed different approaches to answer the challenge in scenario creation, use and management at large. There are several reasons why they cannot immediately be useful in this research. Firstly, while they are developed for specific application domain/area, this research at this stage still stands open to discover potential support areas in the industry. Therefore, this research needs more information from the industrial perspective to assess whether the lessons learnt from the approaches can be applicable. Secondly, except for DIF, the scenarios within these approaches exclude a large part of the dynamic use situations of the product. While this research aims to support scenario creation, use and management in the product design domain, in which the dynamic use situations is a major challenge to tackle. Thirdly, the approaches have been evaluated in case studies and not in a complete cycle of a real design process; their practical value is still unknown. There is a tendency that scenarios are used in an ad-hoc manner specifically in product design practice (as will be verified in Chapter 4). This indicates that the available approaches are not in complete touch with the practice, as also argued by Dorst (2008).

Despite the incompatibility of the existing works with this research, they have pointed out the issues dealing with supporting scenario creation, use and management. By studying them, this research is informed of the concerns that should be taken into consideration while developing the support. Table 3.2 summarizes ideas that can be extracted from the discussed approaches to address the concerns in scenario creation, use and management.

Table 3.2: Lessons learnt from the existing scenario construction models.

\begin{tabular}{|l|l|}
\hline Aim/Concern Partial Solution \\
\hline $\begin{array}{l}\text { Traceability of terms used in } \\
\text { scenarios }\end{array}$ & Glossary (lexicon) \\
\hline $\begin{array}{l}\text { Inambiguity in scenario } \\
\text { descriptions, good readability }\end{array}$ & Rules, e.g. L'ecritoire writing and editing guidelines \\
\hline Completeness and Reusability & Template for writing scenarios \\
\hline $\begin{array}{l}\text { Validity, cogency between } \\
\text { scenarios }\end{array}$ & $\begin{array}{l}\text { Explicit and cross-checked relationships, e.g. DIF aspect } \\
\text { models, SDML }\end{array}$ \\
\hline $\begin{array}{l}\text { Multiple levels of details } \\
\text { (granularity) of scenarios }\end{array}$ & $\begin{array}{l}\text { Iterative scenario building, e.g. L'ecritoire structure of } \\
\text { requirement-goal-scenario, the middle-out integration } \\
\text { scenarios, DIF integration of aspect models }\end{array}$ \\
\hline
\end{tabular}

The aims/concerns in Table 3.2 give the characteristics that should be aimed for in supporting creation, management and use of scenarios. Furthermore, this research still needs to better understand the practical aspects of a product design process, as the target of the support that will be developed. As mentioned in Chapter 2, the existing approaches in scenario-based design often brush aside the complete aspects in design activities (i.e. content, actor, context and process), and focus mainly on enhancing the design process as if it were isolated from the other aspects (Dorst, 2008). As this research aims to develop support that answers the needs from design practice, the inclusion of the aspects is important and the involvement of design practitioners (i.e. the actor) will be necessary. As the first step, this research refines the challenges for supporting scenario creation, use and management (i.e. the content) in the product design domain. 


\subsection{The Challenges: Revisited and Refined for Product Design Domain}

While Chapter 2 has addressed the general challenges of scenario-based design, this section will first elaborate the challenges related to the creation, use and management of scenarios. An interdisciplinary overview has been proposed by Jarke et al (1998), which reviews scenario management from three major disciplines that use scenarios strategic management, human-computer interaction, and software and systems engineering. Their research acknowledges that scenario development, analysis and management are mainly practical processes that depend on creative participation and inputs from individuals. The value of scenarios is that they serve as catalysts for such processes, and not as a rigid methodology. Subsequently Jarke et al (1998) points out Frequently Asked Questions about Scenario-Based Design that indicate directions for future research specifically towards better scenario management (Table 3.3). Although these questions have been asked over a decade ago, they still remain open and are fully relevant with this research which aims to bring scenario-based design into product design practice. This research anticipates similar questions, but independently redefines the questions and seeks out answers specific to the product design domain later in this section. Table 3.3 presents the FAQs along with the possible answers and caveats, completely unaltered from the source.

Table 3.3: Frequently Asked Questions (FAQs) for scenario-based design (Jarke et al., 1998)

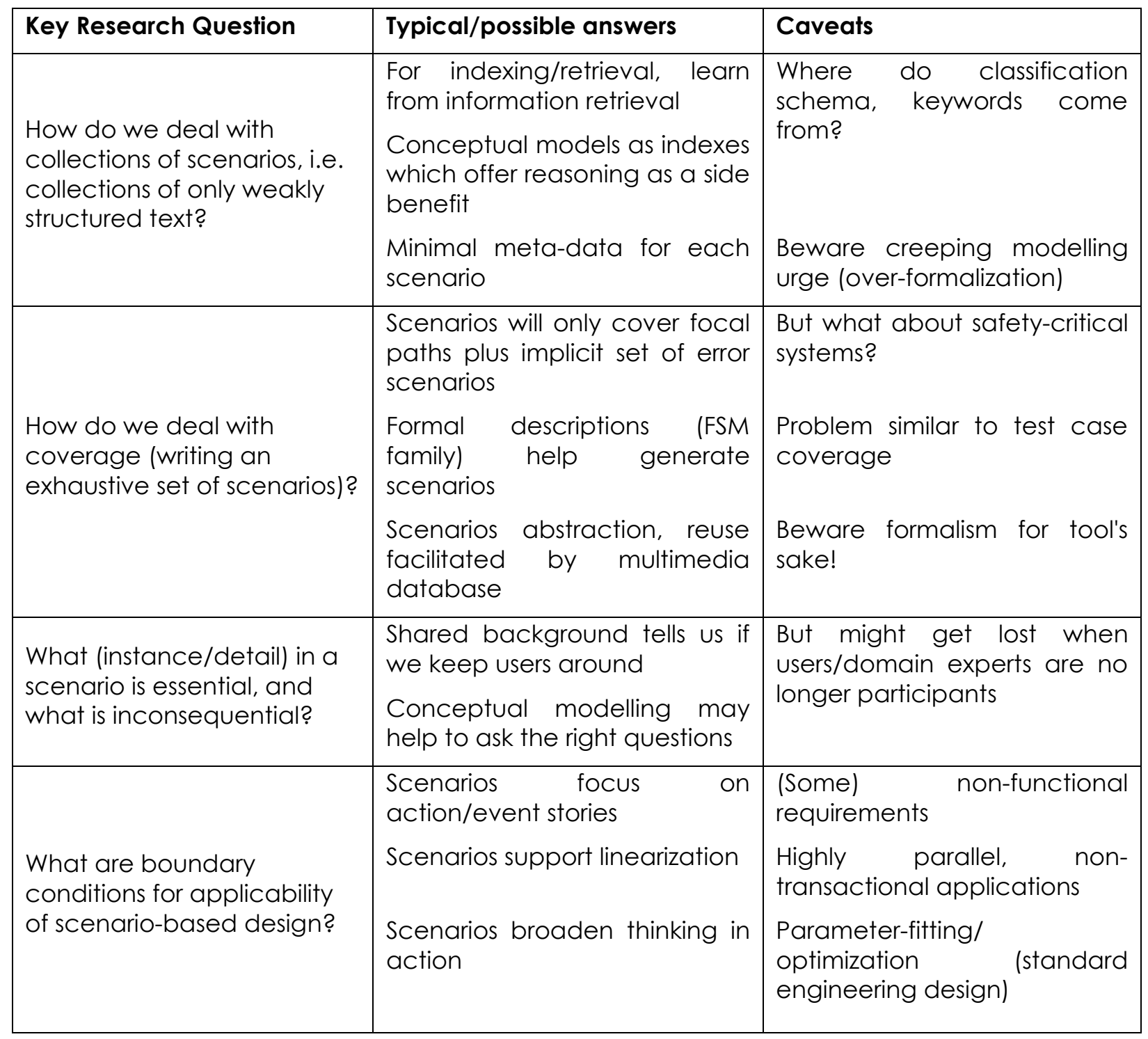


The questions in Table 3.3 are relevant with our findings from literature so far. As an example, on the instance/detail in a scenario, there is still little consensus and the right answer will always depend on the situation. Several case studies on persona ${ }_{4}-$ first coined by Cooper (1999) - have revealed different ways that work best in different organizations (e.g. Blomquist and Arvola, 2002; Pruitt and Grudin, 2003; Ronkko et al., 2004). Concerning the coverage of scenarios, again there will be different answers depending on the necessity. For instance, the use case models can leave out the context of use since it is often predictable, while DIF must address multiple aspects of use to create a holistic approach for product design.

Within the product design domain, the same questions are essentially present and even amplified. Due to the tendency for dynamic use situations, product design needs to take into account more varied design information. A variety of design techniques and tools is required to find out about this information. This results in a more diverse scenario use to accommodate a bigger amount and more complex information, as well as more varied purposes to use scenarios in the design activities. Therefore this research reformulates the questions into a set of topics for clarification and verification through collaboration with industry.

- A concrete guidance that is not too prescriptive: where is the balance? Despite the many available approaches, models, and support tools, in practice scenarios are often used in ad-hoc manner, as also mentioned in Weidenhaupt et al (1998). The other end, an extreme formalization could become tedious and restraining solutions. A strict formal process is likely not fitting in product design practice. The design of tangible consumer products involves a more intuitive and emotional thinking compared to software development which relies mainly on a rational/logical process. There needs to be a methodical guidance indeed, but it should also be informal and flexible enough as to nurture the creativity in the design process.

- A clear overview of scenario use from start to finish of a design project. Despite the potentials, design practice is often discouraged by the uncertainties involved in applying a scenario-based approach. Building scenarios indeed can be a waste of time if the purpose and the future use of the scenarios are not well-defined. A design team needs to have a better idea of what they sign up for by knowing in advance the efforts involved and expected results. The ratio between efforts and results needs to be measurable. For that reason, a more solid framework is required to present a clear overview of the possible scenario use, so that the design team could plan to integrate scenarios into the design process and get an idea of its efficacy. Our scenario roadmap is a building block for such framework (Figure 3.6). It aims to provide a common ground (and common language) to conduct further research with design practitioners.

- Anticipation of the large amount of design information. As this research aims to contribute to the process of designing tangible consumer products, their dynamic use situations are an important element and need to be accounted for. More varied elements in the situations lead to a large amount of design information, which could be chaotic if not organized well. To be able to create and use scenarios with a vision, there needs to be a clear structure to compose the information pieces and the explicit relationships between them. The literature study to understand scenario elements and their interactions has served as a foundation

\footnotetext{
${ }^{4} \mathrm{~A}$ persona is a description of a fictive person that represents a target user group.
} 
to indicate the types of information relevant to user-centred design using scenarios (as illustrated in Figure 3.4).

\subsection{Conclusion}

This chapter has addressed the development of Scenario-Based Design (SBD), and discussed topics related to the research aim to support scenario creation, use and management. From learning from the existing works, a set of topics has been formulated as the refined challenges for Scenario-Based Product Design (SBPD). To develop a support tool for scenario creation, use and management in product design, this research first needs to address the three topics: (1) concrete, non-prescriptive guidance, (2) clear overview of scenario use throughout the design process, and (3) accommodation of a large amount of information.

As this research also aims to make its contribution relevant to the world of practice, a complementary study is required to learn about other aspects of design practice (such as the designers, context and content of the design activities). Such knowledge lies in the design practice. Therefore, this research is going to address these topics in collaboration with industry. The next chapter presents the collaboration process and its results. 
Part II

Action and Reflection 
Chapter 4 - Scenario-Based Design from a Practical Perspective 


\section{Scenario-Based Design from a Practical Perspective}

This research aims to contribute a support tool for scenario creation, use and management that is relevant with the design practice. The knowledge from the design practice is essential to inform this research with a practical perspective. Therefore, collaboration with industry is aimed for. To make the collaboration effective, a set of concrete topics has been formulated in Chapter 3 to be answered together with design practitioners. In this chapter, the term "designers" is used to refer to persons of different functions who are involved in a design project. This generalization reflects on the fact that designing is not only the action of creating product concepts, but also everything else required to deliver the product to the market. Therefore, designers can be ethnographers, market researchers, usability specialists, engineers, managers, or other functions. This assumption however, will be clarified during the inquiries in the design practice.

To involve the design practitioners, scenarios are used as a communication tool. This chapter describes the approaches undertaken to identify problems and solutions with them, and how these approaches make use of scenarios. This initial collaboration process and the findings have been partially published in Anggreeni and van der Voort (2009a; 2009c). This research expects to identify potential areas for support from the collaboration with industry.

\subsection{Collaborator Search}

The researcher conducted informal interviews to get acquainted with design practices and practitioners, especially the ones with interest in scenario-based design. Based on the preliminary interviews with several representatives of design/engineering industry, a Dutch design agency Indes is chosen as a partner for further collaboration. Indes is a small/medium sized company of about 35 employees in The Netherlands and 20 employees in China. It is an ideal collaborator to this research because of the following reasons:

- With small teams working in projects and a flat hierarchy in Indes, there is little bureaucracy in the interaction between designers. Communication within a design team is direct and efficient. The design process is transparent, pragmatic and frugal, adapted from the company's design methodology for each specific project. Therefore, this research can expect an honest and level-headed feedback from this collaboration.

- Indes' product line, among others consumer products for human care, medical cure and user comforts, demands a user-centred approach for market success. Each design project involves the variety of target users because their acceptance towards the products is essential. The approach attempts to discover all possible aspects of product use early on to deliver products with a good usability. With usability as a goal and dynamic use situations as a background, this design practice is a fully relevant application area for scenario-based design.

- The designers at Indes are mostly educated within the Netherlands. With the close ties between the three Technical Universities in The Netherlands (TU Delft, TU Eindhoven and University of Twente), Dutch design education has developed a common platform where educators and researchers interact, cooperate and streamline the education for industrial designers. Therefore, the company can be considered a representative of the current Dutch design practice. Learning the 
practice in the company is expected to give a good idea on the general practice of design companies in the Netherlands. Further steps in this research will however verify this assumption. With the internationally-competitive design industry in the Netherlands, the focus on design practice in the Netherlands will not limit the applicability of this research.

- Lastly, the company already champions scenarios as a part of its user-centred methodology. Everyone in the company realizes the benefits of scenario-based design and wishes for a more coherent approach. There is a mutual interest for collaboration between the company and this research. Therefore, a high-level commitment can be expected from the company.

With information from the preliminary interviews with Indes, further steps for collaboration is planned in the form of workshops. The next section will describe a series of workshops as a means to get direct insight into their specific scenario-based design practice.

\subsection{Workshops}

The workshops at Indes aim to get a general insight into the people working there, their current practice, and how their organization uses scenarios in particular. This research expects to understand the practical application of scenarios as well as the activities/areas that can be improved. On a more detailed level, the workshops have the following objectives:

- To get a better understanding on the designer role in Indes,

- To identify the stakeholders of the proposed support tool, i.e. the people whose practice will be influenced by it, and therefore should be taken into consideration in the design process,

- To understand how scenarios are being used in the company's practice, which includes identifying challenges and other possibilities for applying scenarios,

- To explore the directions and requirements for the form of support to make scenario uses more efficient and effective,

- To verify the scenario use roadmap, which summarizes our understanding on the types of scenario use in a design process (see Figure 3.6).

Two workshops are planned with Indes, each of which involves two experienced industrial designers/engineers. Credited to the transparent design process and direct communication among all project members in Indes, all four participants have experience in user-centred design and using scenarios, although in different levels. Two of them have many years of experience and are in managerial level. Prior to the workshop, an official invitation letter is sent to all participants one week in advance. The letter explains the overview of the program and includes scenarios to describe the fictive case study (see Appendix 1).

As a preparation for the workshops at Indes, three pilot workshops are conducted to select the format that best delivers the objectives within the available time and resources. Each pilot workshop involves two participants. These pilot participants consist of six design researchers with industrial design experiences. Upon learning from the experience of conducting the pilot workshops, the workshop format is reconfigured and fine-tuned. Figure 4.1 gives an overview of the final workshop program.

The findings from the workshops could be idiosyncratic to the practice of Indes. To make sure that the findings are scalable to the design industry at large, they are verified by means of questionnaires to other design practitioners from varied backgrounds and 
work cultures. The combined findings from the workshops and the questionnaires will be discussed in the later sections. The next section addresses the inquiry process using the questionnaire.

Participants:

- Moderator (i.e. the researcher, whose task is to guide the participants to perform the workshop tasks)

- 2 experienced designers (who perform the workshop tasks together)

Programme:

1. Interactive presentation ( 45 minutes)

The presentation aims to familiarize the participants with the research and the scenario-based product design methodology. It introduces the participants to our idea of different scenario uses in the design phases.

\section{A case study (2 hours)}

The case study simulates a fictional design project for which the participants plan a design strategy. The design strategy should be as close as possible to their current practice; at the same time potential scenario uses are identified. The chosen design case is designing a bicycle luggage transporter for a market 5 to 10 years in the future.

1) Revisiting the case study and preparative tasks (15 minutes)

A quick review of the case study. The participants are encouraged to share their experience in regard to the case study by telling stories or other ways they are comfortable with.

\section{2) Customizing a scenario-based design strategy (45 minutes)}

The moderator introduces a partial example of a design strategy to solve the design case. The example shows a set of design techniques that involve scenario uses. The participants customize the design strategy by removing or changing the existing techniques or adding their own techniques. From the resulting design approach, the participants together with the moderator scan and mark the techniques in which scenarios can be useful.

3) Detailing the scenario uses (45 minutes)

The participants choose the most important scenario uses from their design strategy. The moderator guides a discussion about the concrete plan for the scenario uses. The concrete plan should address: Which techniques are used to gather data for creating the scenarios? Who will be involved in the making of scenarios? Who will be the audience of the scenarios? Depending on the target audience, which media of scenario representation is most suitable?

4) Wrap up the case study session (15 minutes)

The moderator discusses with the participants the possible challenges and problems in conducting the scenario-related techniques as proposed in the design strategy. We later focus the discussion on the required functionality of a tool that could support the design activities.

At the end of the workshop, the participants give feedback and suggestions. They also fill in short questionnaires about their personal and professional information. The workshop session is audio-video recorded.

Figure 4.1: The 3-hour workshop programme. 


\subsection{Questionnaire}

During the workshops, several problem areas and directions of support have been explored. The questionnaire aims at verifying the problems in current design practice, as well as probing more details for the directions of support which are most accepted by the design practitioners. The design practitioners are represented by seven experienced industrial designers/engineers, none of whom participated in the workshops. While all of them are familiar with user-centred design, only two persons use scenarios in their daily work practice. The questionnaire uses scenarios and conceptual drawings using Ms PowerPoint to illustrate the problems and potential solutions. Table 4.1 and Figure 4.2 show a part of the scenarios and drawings used in the questionnaire. Appendix 2 lists the questionnaire in full.

Table 4.1: An example of current and future scenario pair in the questionnaire.

Current Practice Scenarios
Please imagine the following situation...
The "bike luggage transporter" design team
is meeting for the first time after the kick-off
meeting. During this period, everyone has
been busy doing research (desk research or
field studies). Therefore, this meeting aims to
be a forum where everyone can share what
he or she has found during the research. And
of course, if there's time left, the team can
discuss what they must do now, how to
move on, etc.

\section{Again, an unproductive meeting}

Before the meeting, designers (individual or in group) prepare presentations to describe their findings within 10-15 minutes time-frame. Most often, this is nowadays done using PowerPoint presentation which will be quite tedious to manage afterwards. Quite often, time runs out before any meaningful discussion gets to the table. When this happens, Mike the project manager (as a representative and member of the design team) and other management will have another meeting, and later on decide what to do next...

\section{Future Practice Scenarios}

Imagine a different situation...

The "bike luggage transporter" design team is meeting for the first time after the kick-off meeting. During this period, everyone has been busy doing research (desk research or field studies) and now the "Scenario Central" application shows a good overview of the users and their use scenarios. Mike the project manager has asked everyone to get acquainted with all the information posted on "Scenario Central". The meeting will discuss what to do next as a team, instead of explaining the design information (which is already registered in "Scenario Central") to one another.

Well-informed designers make a productive meeting

During the meeting, designers are 'empowered' with the well-organized information as they can easily refer to specific scenarios to back up their opinions. Mike suggests a discussion on the user goal "transporting groceries" because it looks promising as a tentative direction. The "Scenario Central" application has a function to filter scenarios based on a specific element. To aid their discussion. Mike uses the filter function to show only information relevant to the goal "transporting groceries" (Figure 4.2). The designers see the overview of problems with current products when their users "transport groceries" and this helps them to focus. 


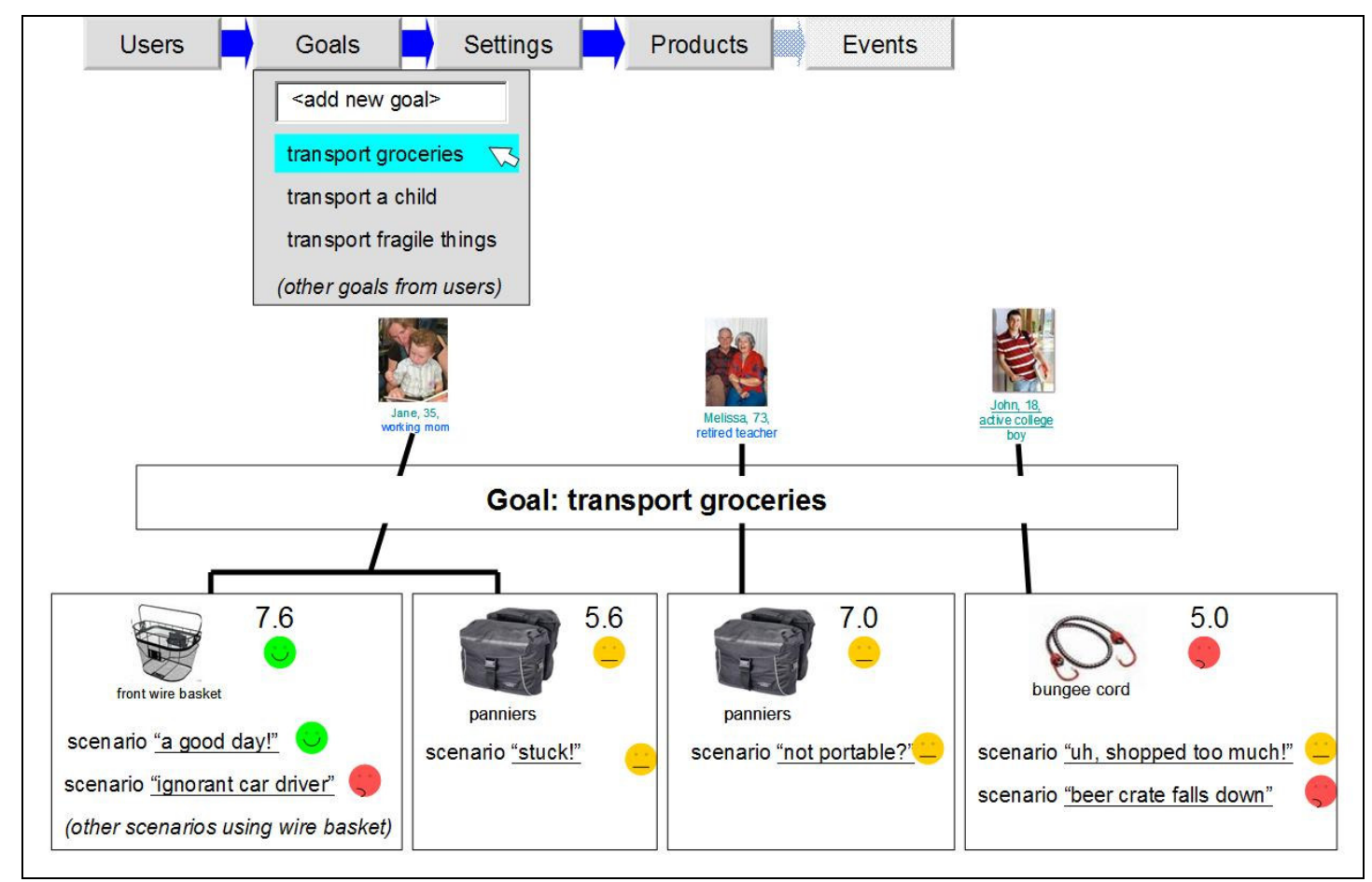

Figure 4.2: Interface of the tool illustrated using PowerPoint drawing; the functionality shows filtering scenarios based on a specific goal "transport groceries".

The scenarios in Table 4.1 describe the problems in current practice and the hypothetical (future) uses with the support tool implemented. While it is easy to agree or disagree with the scenarios, the questionnaire also shows the proposed interfaces and interactions with the tool (Figure 4.2) to engage the respondents. A combination of scenarios and illustrations assists the participants to reflect on their practice. Using these media, the participants are better able to answer the open questions which are aimed to elicit their work practice and their feedback on the proposed tool. The gained insights into the participants' work practice and their inputs for the support tool confirm the findings from the workshop and inform the further steps of this research. The following section will elaborate the findings from both the workshops and the questionnaires to give an overview of scenario use in the current practice.

\subsection{Scenarios in Product Design Practice}

The workshops and questionnaires have informed this research how designers conduct their activities using scenarios, the challenges they face and how they wish to be supported. Three main problem areas in the design practice have been identified. Through the discovery of these problem areas, the scenario use roadmap (Figure 3.6) has been verified as the designers were able to relate their own scenario practice to the roadmap during the discussion. The following subsections will elaborate the identified problem areas in more details.

\subsubsection{Documenting design knowledge}

The current practice: Before a project is started, the designers build intensive contact with their clients to get the business requirements right. Once the project is defined and throughout the process, the designers compile their design information from contacts with potential end-users, observations on competitor products and close investigations of established standards (e.g. safety or ergonomics). They also employ design techniques such as ethnography, observation, interview, focus groups and participatory design methods to get a good insight from the user perspective. 
Especially at the beginning of the design process, the design information tends to explode because the significance of the information pieces cannot yet be determined. As a result, the designers take in all the information so that they do not miss anything that may contribute to their decisions later on, which may result in the information lacking organization.

The challenges: Within the development of a complex product, this early phase will pose challenges in selecting the information relevant to the design case beforehand. The various design techniques often lead to varied media of documentation, which often results in information in a large amount, scattered, and generally overwhelming to the designers. This information, a.o. the users and their characteristics, their goals, products they are using, etc, contains the right ingredients for scenario building. Designers are aware of it, yet as they are finding out more information it is rarely the right time to start making a structured documentation about their findings. The task to document all the gathered information might already take an enormous effort and time. While it is getting more streamlined later on, the beginning of a process is usually associated with ad-hoc documenting activities. There could be a lot of efforts saved if the most relevant information are identified early and gathered first. Designers within a team would therefore benefit from a more structured manner to collaboratively document their findings; which can be regarded as an investment for future easy access to their design knowledge.

\subsubsection{Proposing solutions and identifying requirements}

The current practice: Based on the information from the previous phase, designers pay special attention to important use situations that give shape to solutions. They propose ideas and develop concepts to answer user problems as discovered during the analysis phase. While the ideas or concepts are still abstract, the designers find it essential to be able to communicate them, for instance to discuss them with their peers or stakeholders. At this stage, the ideas or concepts are often illustrated in drawings/sketches/mock-ups, with their supposed uses expressed in impromptu stories. Being able to communicate these early ideas and concepts helps the designers to reflect on their suitability and critical aspects. These stories (scenarios) however, are rarely documented in the project because they are considered not important. Furthermore, when new use situations are discovered, they are often not documented as scenarios but rather are translated directly into requirements or risks. These requirements and risks are on a different level than scenarios. They aim to be brief and formal.

The challenges: The organization of all design information is always a challenge. Most design practices eventually use a formal tool or tools to deliver reliable results to the stakeholders. However, the process prior to this formal step is largely unguided. Switching between the creative analysis/inquiry and design/synthesis activities and the rigor of writing formal requirements/risks can be experienced as disruptive by the designers. Furthermore, it may be that due to a lack of organization, a requirement or a risk could not be traced back to its rationales. These rationales could be in the form of scenarios; and they might not be documented because they were initially considered unimportant.

\subsubsection{Evaluating design concepts}

The current practice: While idea generation is intuitive and rarely performed in a structured manner, rationality should not be compromised. Any design concept must lead to at least a sufficient performance in all important use situations. Therefore, requirements and/or risks are often used as a "checklist" for testing product concepts. 
However, the requirements/risks might miss the complete context for the testing. A valid test setup needs to explicitly include the elements of the use situations (e.g. setting, user's state of mind, existence of any distraction, etc). The format of formal requirements/risks does not consistently include all these elements. In practice, artefacts or documentation from the previous phases are sometimes revisited to accurately reconstruct the use situations.

The challenges: When designing a complex product, the formal requirements/risks as well as scenarios easily grow more complex. The norm to evaluate a product concept is by running it through the list of requirements/risks. Although it is reliable and the result is rigorous, it is also mentally exhausting to the designers. Furthermore, when changes are made in the concept, the designers may not be aware which other requirements/risks/scenarios should be retested. Whether the requirements/risks/scenarios have covered all of possible use situations, in other word complete, is rarely known. Product testing often uncovers more unexpected use situations, which then need to be integrated into the existing list of requirements/risks/scenarios. This situation demands flexibility from the designers, and they will appreciate support to better cope with it.

Summarizing, design activities in different phases often deal with challenges concerning the organization of information. Scenarios are present in the activities, but often not documented explicitly as an official part of the project due to the effort needed to create and organize them. The design practice prefers to focus more on formal requirements/risks because they are perceived as more reliable and deliverable towards the clients/stakeholders. Nevertheless, requirements (including risks) that are connected to their rationales, i.e. scenarios, could inspire designers in the rigor of testing. The presence of scenarios is recognized by the designers, as they have been able to reflect on the different purposes of scenarios as proposed in the scenario use roadmap (Figure 3.6). Table 4.2 summarizes the association the designers make with their own scenario practice.

Table 4.2: Scenario usages commonly occurring in the design practice.

\begin{tabular}{|c|c|}
\hline CURRENT & FUTURE \\
\hline $\begin{array}{c}\text { Actual practice scenarios } \\
\text { register observations and interviews; the use } \\
\text { situations might be fragmented or not } \\
\text { complete }\end{array}$ & $\begin{array}{c}\text { Future practice scenarios } \\
\text { capture design directions to } \\
\text { communicate within the design team } \\
\text { and with other stakeholders; rough } \\
\text { sketches are sometimes used to illustrate } \\
\text { future use situations }\end{array}$ \\
\hline $\begin{array}{l}\text { Possible problem scenarios } \\
\text { what could go wrong during product use; } \\
\text { designers criticize their concepts and peer- } \\
\text { review each other's work when needed }\end{array}$ & $\begin{array}{c}\text { Interaction scenarios } \\
\text { (detailed) sketches of product use; } \\
\text { scenarios are explicit and expressed in } \\
\text { ad-hoc manner (e.g. annotation on the } \\
\text { sketches, oral stories, gestures); thus } \\
\text { documentation is not reliable }\end{array}$ \\
\hline $\begin{array}{l}\text { Validation } \\
\text { ensuring product's success in all possible use } \\
\text { future situations); explicit and documented be }\end{array}$ & $\begin{array}{l}\text { narios } \\
\text { enarios (according to both current and }\end{array}$ \\
\hline
\end{tabular}


The involvement of design practitioners through workshops and questionnaires has informed this research the reality of scenario-based design and its practical challenges. Although the initial findings are predominately based on the practice at Indes, the questionnaire responses have revealed that the problems are familiar across different design practices. The findings consistently show that scenarios are indeed an inherent part of design; designers as problem solvers always have them in mind when analyzing, designing and evaluating product ideas or concepts. However, this is mostly done sporadically as a part of the creative process, and the resulting artefacts could be difficult to sustain for later uses. To address these challenges, support can be in the form of integrating scenarios within the design process. The next section elaborates the criteria for a framework that guides the identification/creation, use and organization of scenarios within the design process.

\subsection{Criteria for a Framework for Scenario Creation and Use}

The framework aims to encourage designers to adopt a more structured approach in integrating scenarios in the design process. A set of criteria for support is proposed based on the possibilities of scenario-based design and the findings from the design practice so far. The criteria describe requirements for the support tool that are expected to answer the problem areas.

\subsubsection{Documenting design information as scenario elements}

A template or structure is required for storing design information in an organized yet also flexible way. In Chapter 3, the necessary information which serves as natural building blocks for scenarios has been identified. This information can be referred to as scenario elements (see Figure 3.3). Especially in complex design projects, the dynamic use situations of the products stress the need for such structure even more. These scenario elements could guide designers into identifying and making sure a good coverage of the aspects of use situations.

\subsubsection{Creating scenarios to make sense of the information}

Some scenario elements can be more important than others. Think of frequent users compared to occasional users of the product, or a critical event that has a high probability to occur during the product use. Identifying the most important elements is an essential step to construct meaningful scenarios. The previous requirement proposes a structured documentation using scenario elements as template. To build upon it, scenario elements can be combined to provide a starting point for scenarios. To prioritize the creation of the most important scenarios, the tool could assist the designers by showing the most important, or the most relevant combinations of scenario elements. By being able to use the scenario elements to build scenarios, designers can be motivated to maintain the documented information. Scenarios can inspire requirements, and there should be a possibility to connect scenarios with requirements. By making explicit the relationships between information, the designers are more aware of them. This will emphasize the value of using scenarios throughout the design process.

\subsubsection{Sustaining scenario uses as an integrated part of the design process}

The previous requirement is expected to improve the accessibility and connectivity of scenarios with other design information as well as requirements. Support is also necessary in the retrieval of scenarios relevant for particular purposes. For example, the tool could suggest a set of scenarios for validating specific product parts or functions, or a set of misuse scenarios for extreme testing. Consequently it should also be possible to keep track and administer scenario uses in the design project. By making explicit how scenarios relate to other information, and for which purposes they could be useful, 
design decisions could be traced back to their rationales more easily. Eventually, a collaborative team effort could sustain informal, yet methodical documentation of information that will benefit the project in the long term (for instance, formal reports do not have to be from scratch).

Summarizing, this section has proposed a set of criteria for a support tool for creating, using and managing scenarios in a design process. These criteria are directly related with the problem areas encountered in design practice. A support tool that fits the criteria, i.e. fulfilling the requirements, is expected to be a framework for a better scenario-based design practice. On a practical level, it is also essential to already imagine the support tool in its proper context, i.e. the possible setting and the people who are likely to interact with it. Throughout the contact with the design practitioners, a clearer picture has formed on who would be involved in the implementation and use of such a support tool. While not all of them are going to use the support tool with the same intensity, these stakeholders are generally introduced as designated actors within a particular project using the support tool, and will be explained in more details in the next section.

\subsection{Actors of the Support Tool}

In the introduction of this chapter, a generic term "designer" is posed to capture anyone involved in a design project independent of his or her function. One common characteristic is that they have a common interest and goal to make the design project successful. To be able to realize the functionality, it is important to understand the context of a design project in terms of the people involved and their roles. Within the context of Indes, several actors have been identified. Although a different project might involve a different composition of actors, they are considered generic enough and can be recognized in most project settings. The following list introduces the actors, and summarizes their roles and interests in a design project:

- Project manager (PM) is the one who manages the course of a design project. During the initial stage of the project, PM is responsible for defining an agreement with the client and possibly other stakeholders on subjects such as project timeframe and budget. An important part of this agreement is the design approach which the design team will use to accomplish the assignment. As it directly relates to cost, the client could wish to remove parts of the proposed approach to cut expenses.

- Client is the one giving an assignment to the design company. The client has the biggest interest in the design project in terms of business profit. During the initial stage of the project, the client defines a clear orientation and project scope with the design team. Additionally, he has rich information, for instance about the target users/buyers, a rough product idea and relevant technologies, potential sources of information (e.g. people, documents, standards or regulations) and competitor products of similar line.

- End-users or users are the persons or professionals who are going to use the product. They have the domain skill and knowledge that is resourceful towards the design. The design team needs to obtain this tacit information to be able to design a useful product. A variety of user-centred and participatory design techniques/methods can be used to involve these people in design activities.

- Designers (also referred to as developers sometimes) are members of a design team and could be of diverse functions such as engineers, software developers, usability specialists, business analysts, ergonomic experts, testers (Quality Assurance), etc. They conduct design activities to fulfil the assignment as agreed by the PM and 
client. Even with the design approach already defined, designers are more stimulated when they have freedom to employ their multitude of skills, creativity and perspectives in executing design techniques. Therefore despite their different backgrounds and functions, these creative problem-solvers in a design project can be referred to as simply designers. Although in different contexts, designers can have more specified titles or functions, this versatile "problem-solvers" profile reflects the context of Indes better.

These actors are acknowledged as a part of the framework for scenario creation and use in product design. The realization of the functionality should consistently consider them as a part of the solution. The solution, in turn, should assist these actors in achieving their goals within a design project.

\subsection{Conclusion}

This chapter has described the approaches taken to make this research relevant with industry by collaborating with design practitioners. By getting their insights and perspectives on scenario use in practice, specific problem areas, which are also potential support areas for this research, have been identified. Furthermore the stakeholders or actors of the support tool have been recognized, and will be consistently included as an active part of the framework for scenario creation and use. A set of criteria for a support tool has been proposed to answer the problems, and in the process also assist these actors to achieve their goals in the design process. Table 4.3 elaborates these criteria further into functionality from the perspective of the designers.

Table 4.3: A summary of the designers' goals and proposed tool functionality.

\begin{tabular}{|c|c|c|}
\hline Goal & & Requirement for Functionality \\
\hline $\begin{array}{l}\text { Gather, register, organize relevant design } \\
\text { information efficiently (going broad, } \\
\text { taking in information) }\end{array}$ & $\leftrightarrow$ & $\begin{array}{l}\text { A "template" for documenting design } \\
\text { information based on scenario elements } \\
\text { and a "toolbox" to create scenarios using } \\
\text { the information as building blocks }\end{array}$ \\
\hline $\begin{array}{l}\text { Be in the know of what information is } \\
\text { available, especially for choosing the } \\
\text { important/relevant ones for making } \\
\text { meaningful scenarios (from the extensive } \\
\text { information, how to narrow it down to fulfill } \\
\text { a goal) }\end{array}$ & $\leftrightarrow$ & $\begin{array}{l}\text { A visualized overview of scenario } \\
\text { elements and their relation with one } \\
\text { another and with scenarios }\end{array}$ \\
\hline $\begin{array}{l}\text { Coherent and concrete scenarios, that } \\
\text { explicitly contribute to the design process } \\
\text { (acknowledging that scenarios are only } \\
\text { an in-between artefact towards the } \\
\text { product) }\end{array}$ & $\leftarrow$ & $\begin{array}{l}\text { Explicit relations between scenarios and } \\
\text { product requirements }\end{array}$ \\
\hline $\begin{array}{l}\text { Action and reflection, a quick evaluation } \\
\text { of concepts/ideas (without being a } \\
\text { hindrance to designers' creativity) }\end{array}$ & $\leftrightarrow$ & $\begin{array}{l}\text { Easy retrieval of scenarios to provide } \\
\text { reliable rationales } \\
\text { (The tool can be extended as a "wise } \\
\text { wizard" that suggests to designers the } \\
\text { scenarios which are potential for specific } \\
\text { purpose.) }\end{array}$ \\
\hline $\begin{array}{l}\text { Communicating scenarios to other } \\
\text { stakeholders for various purposes (e.g. } \\
\text { testing, marketing, brainstorming) }\end{array}$ & $\leftrightarrow$ & $\begin{array}{l}\text { Narrative as the medium of scenario } \\
\text { representation, which offers a flexibility to } \\
\text { extend scenarios to different media (e.g. } \\
\text { storyboard, role play). }\end{array}$ \\
\hline
\end{tabular}


In Chapter 3 three general challenges of supporting Scenario-Based Product Design (SBPD) have been recognized. The discovered problem areas in practice have confirmed these challenges. Furthermore, the proposed criteria for the support tool are providing relevant answers to them. The form of support as a tool (instead of a methodology, for instance) gives designers a flexibility to choose whether to use it or not. The support tool guides scenario creation by suggesting a template, and not prescribing steps; thus being at once concrete and flexible. The consistent guidance from the start of documenting design information, building scenarios, extracting requirements, and managing all the information, is expected to give an overview of scenario uses in a scenario-based design process. Lastly, by acknowledging that some scenario elements and scenarios are more important than others, the tool can help designers skim through information and prioritize the important ones. Furthermore, the explicit relationships between information could be used by designers to find related information, and eventually deal better with a large amount of design information.

The collaboration with the design practice has informed this research a more complete view about the aspects of design activities, i.e. the actor/designer, context, content and process, according to Dorst (2008). With requirements that are relevant with both literature and practice, functionality of the tool has been proposed and presented in Table 4.3. Based on the requirements for the tool functionality, conceptual designs are developed in iterative steps and evaluated, as will be explained in the next chapter. 



\section{Conceptual Design}

Chapter 4 has proposed functionality for the support tool based on the requirements which have been defined from the theoretical as well as practical perspectives. The functionality becomes the foundation for the conceptual design of the support tool. With the different actors that may have different reasons for interacting with the tool, the description of the concept will focus on its use from the perspective of the actors. This is realized by the use of use case diagrams. For the purpose of evaluation with the designers, scenarios and drawings are used to depict the future use of the support tool. The idea presented in this chapter has been published in Anggreeni and van der Voort (2009b). This chapter describes the development process of the concept and its evaluation with Indes designers.

\subsection{Use Cases of the Support Tool}

To capture the functionality overview of the support tool, UML use case diagrams are used. A use case diagram depicts actors, use cases and associations between them. The actors have been introduced in the previous chapter, as comprising of designer, project manager, user, and client. They are an important part of the use case diagrams, since their goals define the functionality of the tool. The use cases describe the goals of the actors within the scope of the functionality that the support tool provides. If an actor supplies information to, initiates, or receives information as a result of a use case, then there is an association between the actor and the use case. Use cases can have dependencies - extend, include and inheritance (Ambler, 2001-2009). Figure 5.1 shows example of a use case with these dependencies.

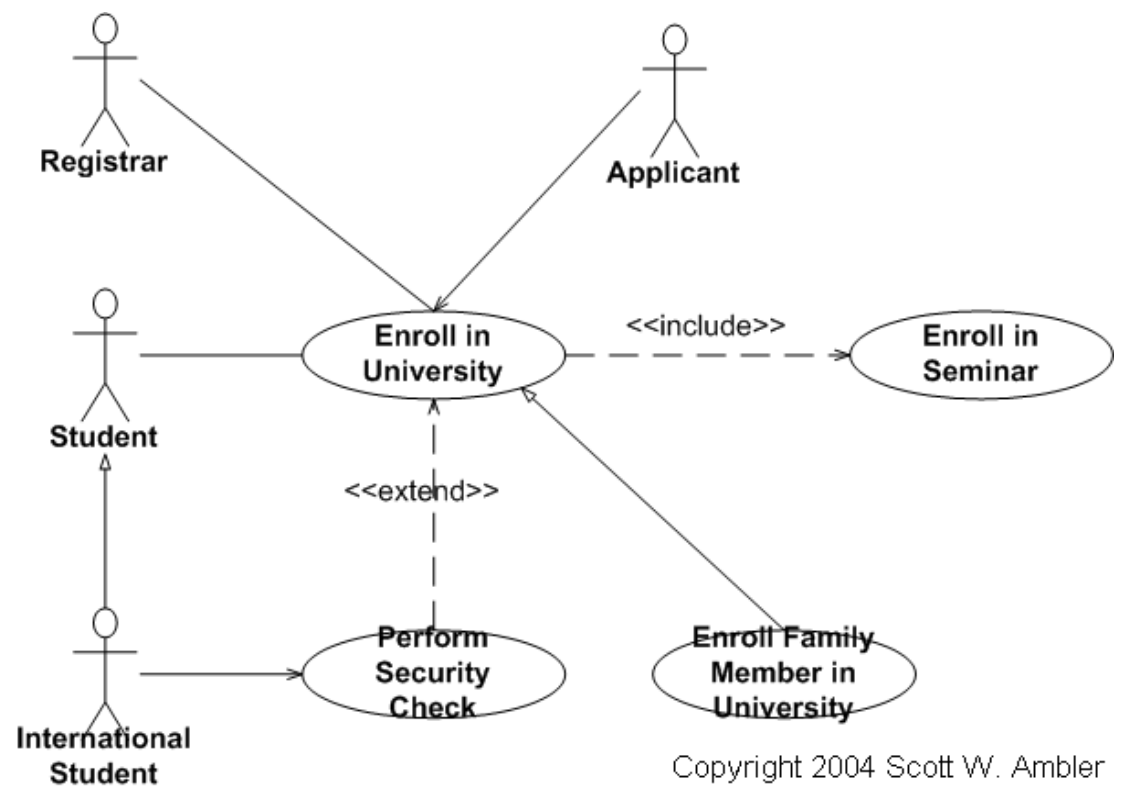

Figure 5.1: An example use case "Enrol in university"5.

An extend dependency is a generalization relationship where an extending use case continues the behaviour of a base use case. Therefore if an extending use case $X$ extends a base use case $Y$, it indicates that $X$ is effectively an alternate course of $Y$; $X$

\footnotetext{
${ }^{5}$ Image courtesy of http://www.agilemodeling.com/essays/useCaseReuse.htm. Retrieved on 21 July 2010.
} 
may initiate $Y$. Another way to look at it, extend relationships are the equivalent of a "hardware interrupt" because it is not known when or if the extending use case will be invoked (they are conditional). An include dependency is a generalization relationship that denotes the inclusion of the behaviour described by another use case. If $X$ includes $Y$, it means that task $X$ has a subtask $Y$; task $Y$ will be completed at least once in the process of completing task $X$. Include relationships can be seen as an equivalent of a procedural call. Lastly, an inheriting use case means that it would completely replace one or more of the courses of action of the inherited use case. If $X$ inherits $Y, X$ is basically a special case or a more specific version of $Y$. To apply this basic knowledge on UML use case diagrams, Figure 5.2 shows the top-level functionality of the support tool as a use case diagram.

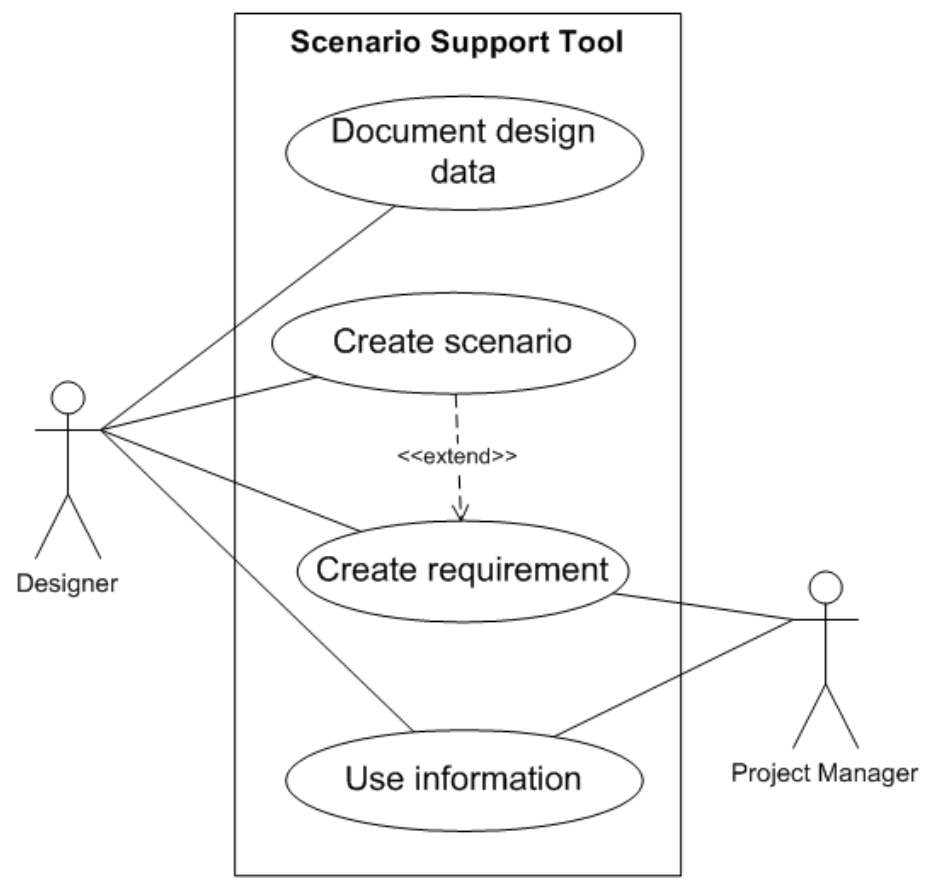

Figure 5.2: Use Case Diagram of the support tool; notice that use case "create requirement" may be initiated from another use case "create scenario".

From top to bottom, the use cases present a chronological order of a design process using scenarios. Different actors interact with the support tool to perform different tasks, which the use cases represent. Each of them can be further analyzed into a more detailed diagram. The rest of this section will go through each use case into more details.

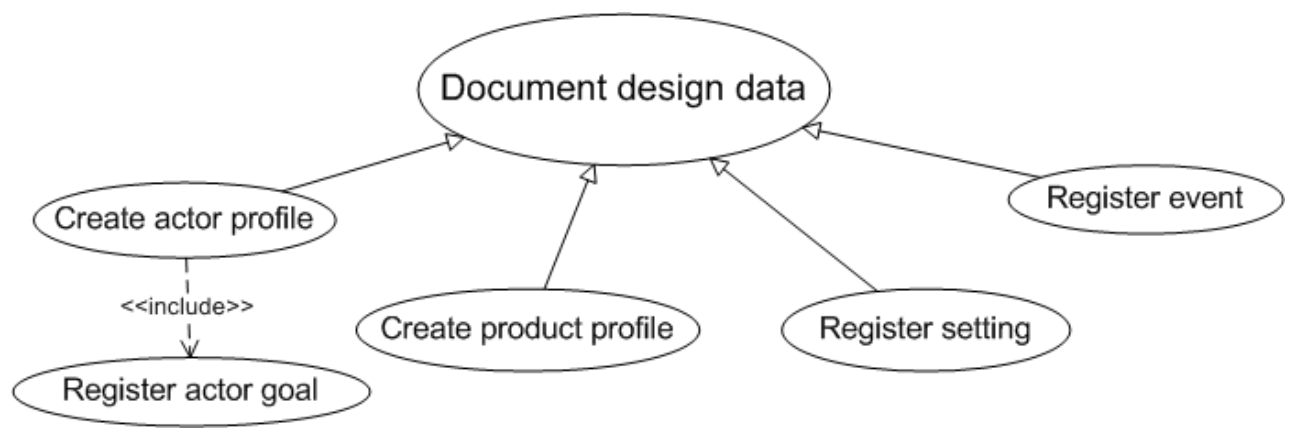

Figure 5.3: "Document design data" consists of several use cases for different types of information. 
Use case "document design data" is the foundation for the rest of the functionality. The later step for scenario building would first need necessary data such as actors, actor goals, products, settings, and events (Figure 3.3 provides an overview of these scenario elements). Documenting design data therefore comprises creating actor profiles, creating product profiles, and registering other scenario elements such as events and settings. During the creation of an actor profile, it should also be possible to register the goals of the particular actor.

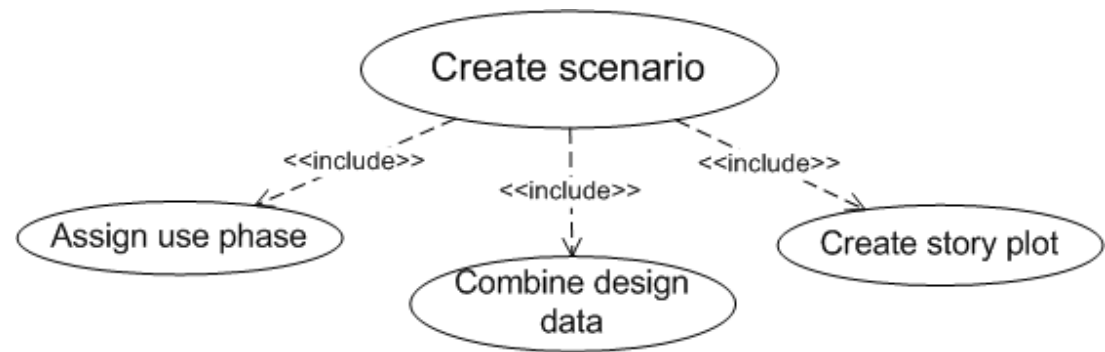

Figure 5.4: "Create scenario" is done in three steps.

Writing scenarios relies on the condition that design data have been documented using the support tool. A scenario starts with an associated use phase, i.e. where in the product life cycle the scenario takes place. In the next subtask, the already documented design data could be a source of inspiration for identifying potential scenarios. Each scenario refers to a combination of design data as its starting elements. The choice of elements then triggers the story plots.

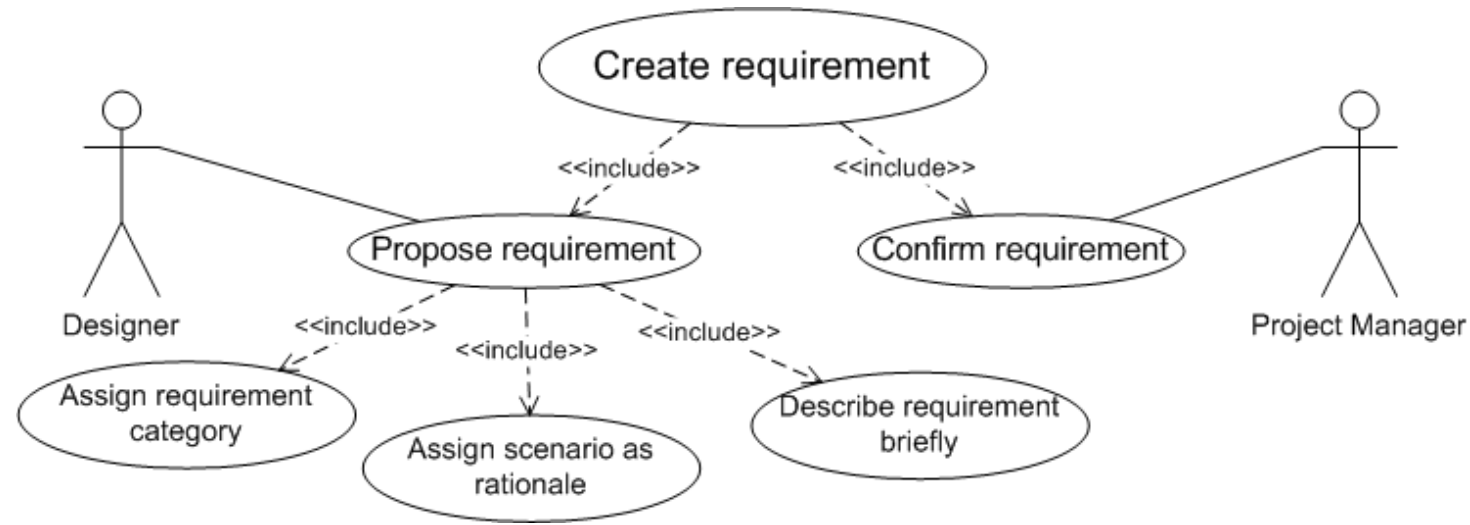

Figure 5.5: "Create requirement" exists on two different levels: the proposal and the confirmation.

Requirements are an important result of a design inquiry process, as they will be the foundation for further design activities. These requirements grow, evolve and maintained throughout the design process as new insights are gained. As a designer identifies a requirement, he or she can categorize and describe it briefly while providing scenario(s) as a rationale. As shown in Figure 5.2, it should also be possible to extract requirements from scenarios. In this case, the scenarios need to be created first. To keep the requirement list solid; the requirement as proposed by the designer is still considered tentative. Depending on the company's practice, it is suggested that the tentative requirement becomes official only after the project manager confirms it. 


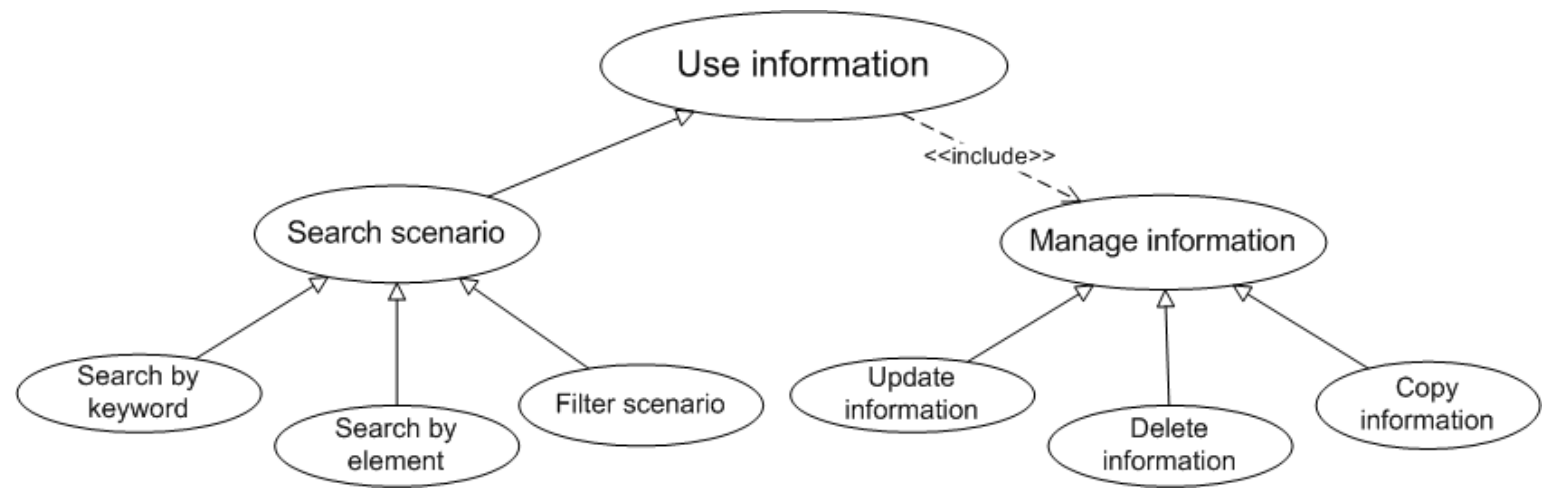

Figure 5.6: For designers to be able to "use information", scenarios as a specific part of the information need to be searchable, and all information should be easily managed.

Scenarios are created to combine the various aspects of use situations as represented in the design data. Requirements are created for the specific different purpose of creating the design solution. Both are evolving together throughout the design process. While the use of available information is planned by the design team itself, the support tool could assist the process by providing easy access to the information. Scenarios are the most interesting pieces information as they are the middle ground between the raw design data and the more formal information such as requirements. Therefore, they are expected to be used heavily in the design process. Different ways to assist in finding scenarios are recognized, e.g. searching by keywords or elements, and filtering out irrelevant scenarios (manually by the designer). To maintain the accessibility and coherence of information, the designer needs to manage all the information including design data, scenarios and requirements. Therefore a possibility to perform the basic update, delete and copy operation is indispensable.

To relate these proposed use cases with the phases in a design process, figure 5.7 illustrates a direction of working with scenarios that is more guided in the processing of information and is in harmony with the natural flow of a design practice. The support tool is expected to aid the loose ends of information processing by making use of scenarios. 


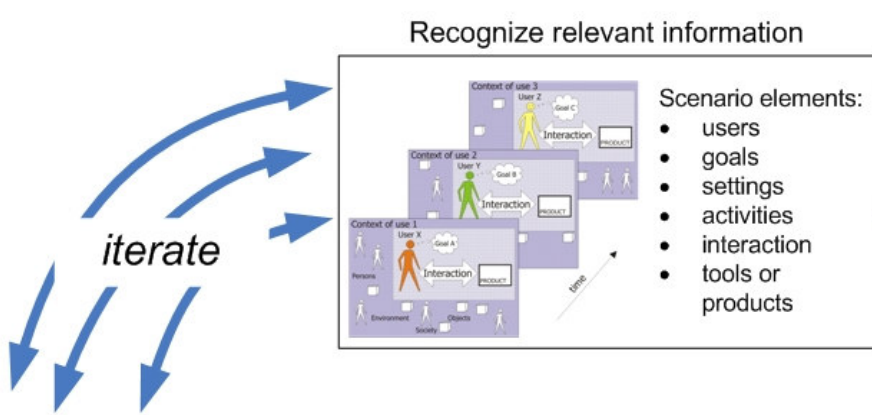

Validation using scenarios
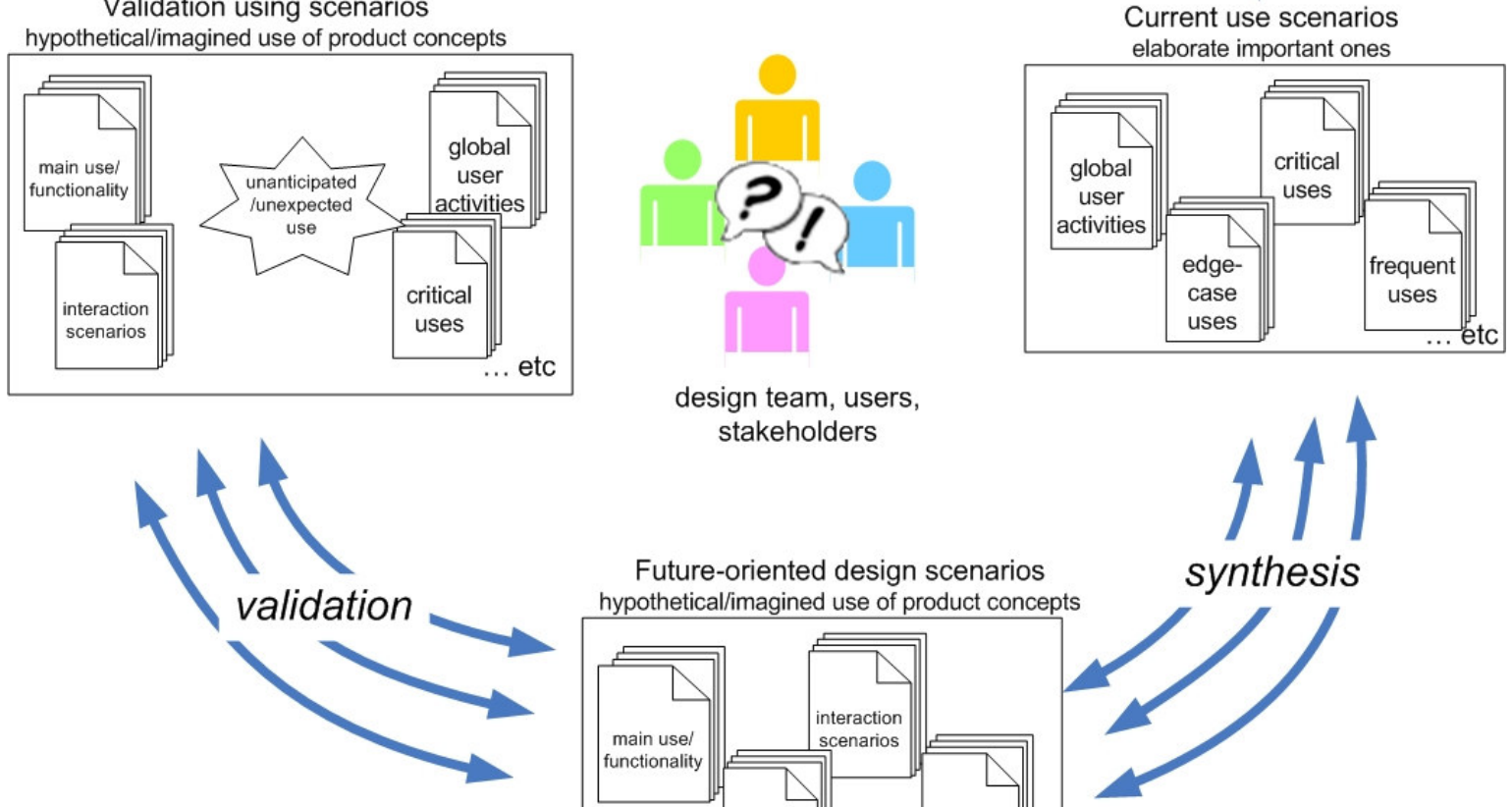

design team, users,

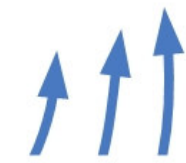

Future-oriented design scenarios synthesis hypothetical/imagined use of product concepts

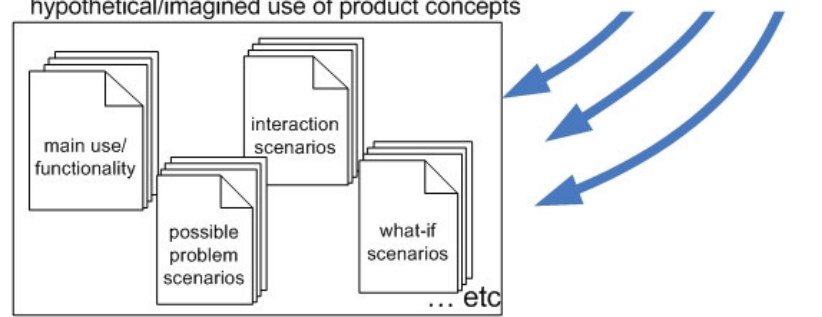

Figure 5.7: An iterative cycle of scenario creation and use in our envisioned design process with the support tool.

Summarizing, the use case diagrams have laid out the foundation for the conceptual design of the tool. The tool aims to support the creation and use of scenarios, and therefore integrates scenarios better in a design process as illustrated in Figure 5.7. The next steps in this conceptual design phase will focus on the basic interaction and interface that would realize the identified use cases. The next section explains the media which are used to develop the basic interaction and interface of the support tool.

\subsection{Media of Development}

Some initial ideas for the support tool have been presented in the explorative questionnaire to probe for possible solutions (as described in Chapter 4). From the feedback received from the respondents (i.e. designers from varied practices/companies), these ideas are refined into more concrete concepts with realistic interface and interaction that can be further implemented as prototypes. The concepts are developed in small iterations. The first prototypes are sketches made on paper by the researcher. The paper prototype is mainly used for the researcher's own reflection and informal discussions with peers. Since these prototypes will later serve to communicate the ideas with the designers, they need to be representable and most importantly, readable. Therefore, after a certain level of confidence, the paper sketches are ported to digital drawings using Microsoft Visio. Figure 5.8 shows the 
transformation from paper to Visio prototype of the 'create actor (user) profile' function. Throughout the presentation of the prototypes, the same fictive design case is used, i.e. the design of bicycle luggage transporter.

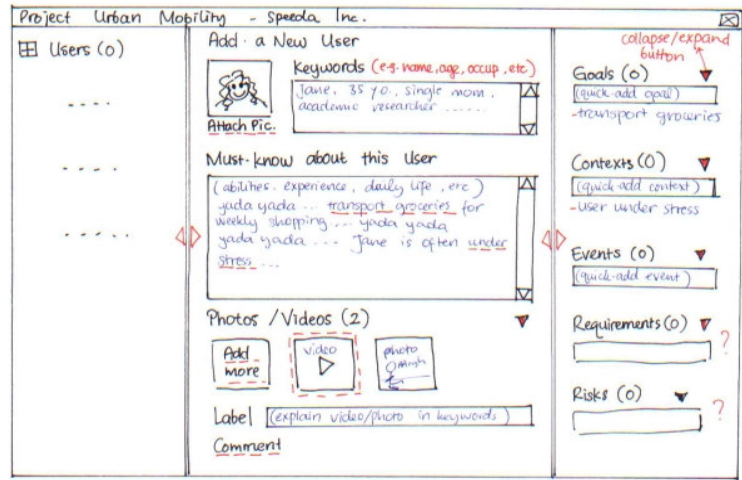

Paper prototype

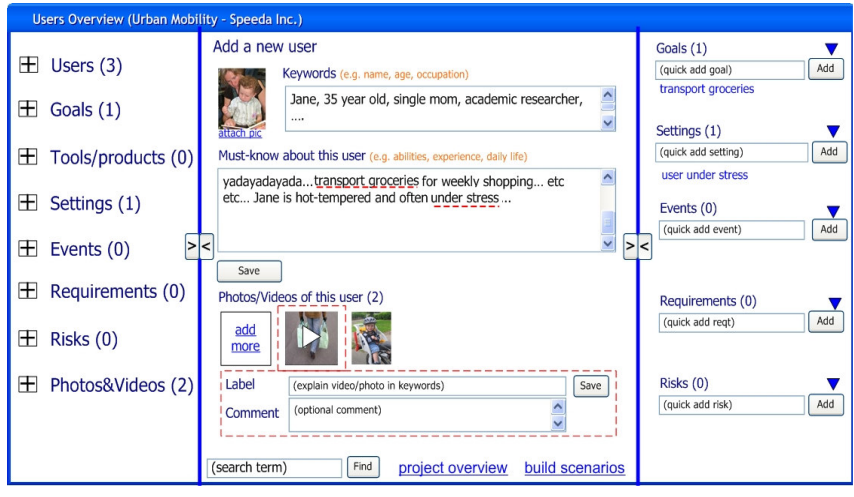

Visio prototype

Figure 5.8: The transformation from early paper prototype to digital prototype using Visio.

The Visio prototype, although looking presentable, is still static and not explanatory enough about how a designer can interact with it. To implement a rough working prototype that includes the interaction, on the other hand, would demand a lot of effort and time and impose a bigger risk in the case that the ideas are not accepted by the designers. Therefore for the evaluation with the designers, the Visio prototype is complemented with scenarios that describe its context of use, and animated using PowerPoint to illustrate the supposed interaction with the tool. The next section describes our approach to evaluate this conceptual design of the support tool.

\subsection{Evaluation with Indes}

Since the functionality has been developed with the collaboration of mainly Indes designers, it is a natural choice to involve them again in the evaluation of the conceptual ideas. This early evaluation aims to get qualitative feedback and more ideas from the designers. To achieve this aim, a focus group meeting of one hour is planned. All Indes designers who are interested in the topic of supporting scenario creation and use are invited; around 10 - 12 people are present in the meeting. At this point, although Indes is main collaborator of this research, most of the designers are new to the research subject. Therefore, the focus group meeting also includes an introduction to the research topic on supporting the scenario creation and use within a scenario-based product design process. The main part of the meeting is the "storytelling" about the proposed support tool. The focus group meeting is then concluded with an open discussion.

The storytelling is performed by means of the Visio prototype and a set of future use scenarios. To show the supposed interaction with the support tool, the Visio prototype is animated using PowerPoint. At the start of the session, printouts of the Visio drawings are distributed and the designers are instructed that they could freely annotate the printouts with their suggestions/feedback during the storytelling. First, an introduction scenario is read aloud to explain the context of the project for which the support tool is intended (Figure 5.9). Afterwards, the proposed functions of the support tool are expressed by reading the use scenarios aloud. A PowerPoint presentation shows the Visio prototype and animates the interactions as the scenarios are being read. To give a better idea of the evaluation session, the next section elaborates the means of the evaluation, i.e. the conceptual prototype in the form of Visio drawings and scenarios. 


\subsection{Conceptual Prototype and Scenarios}

The following description on the conceptual prototype and scenarios are actual to the one used in the evaluation. As mentioned in the evaluation approach, an introduction scenario is created to expose the designers to the context for which the support tool is intended. As it is based on the inquiry in Indes practice (in Chapter 4), this introduction scenario helps the designers to reflect on their own familiar experiences. Figure 5.9 tells the introduction scenario, followed by an illustration of the tool's welcome screen and first use scenario in Figure 5.10.

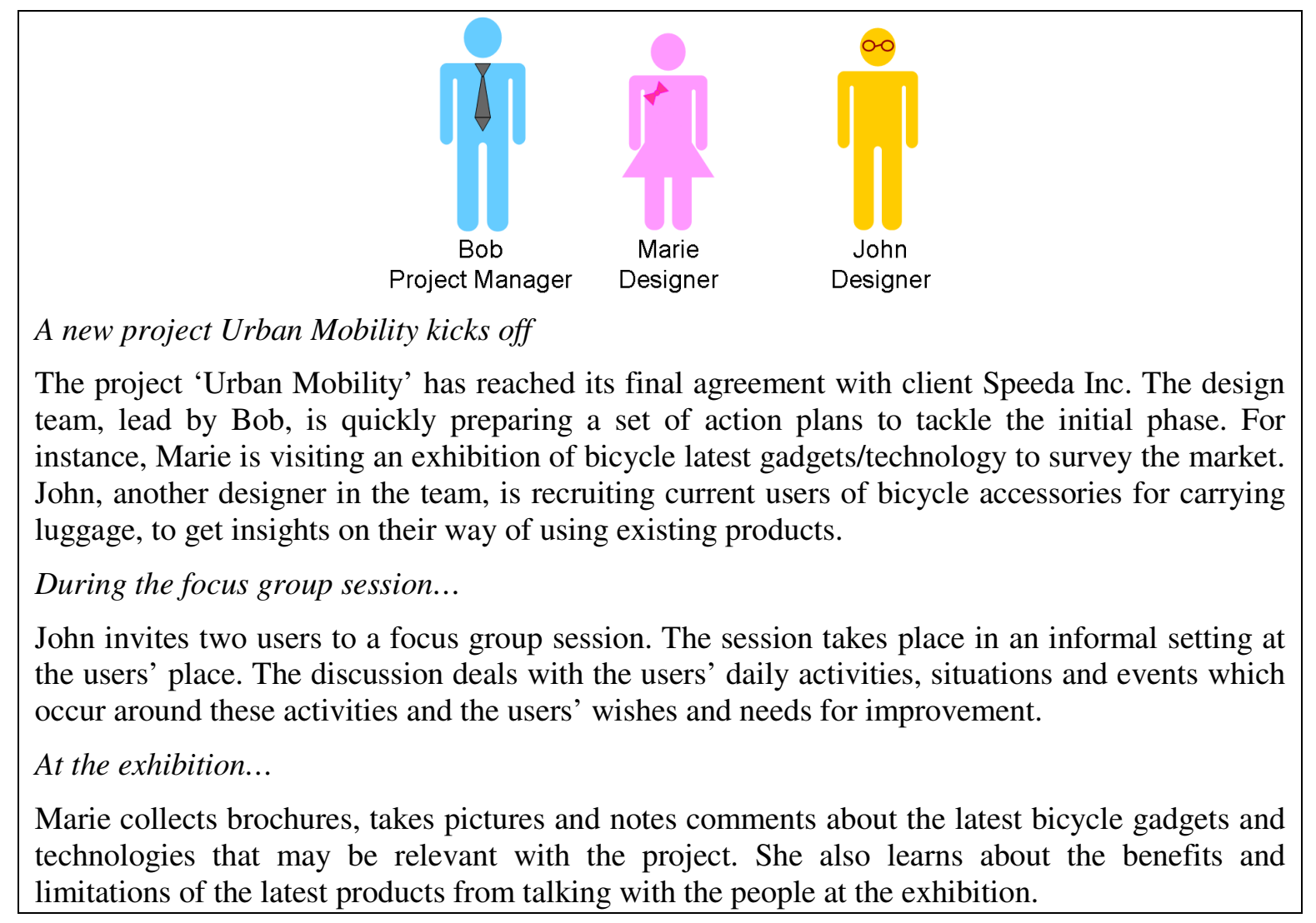

Figure 5.9: An introduction scenario to explain the context for which the support tool is being used. 


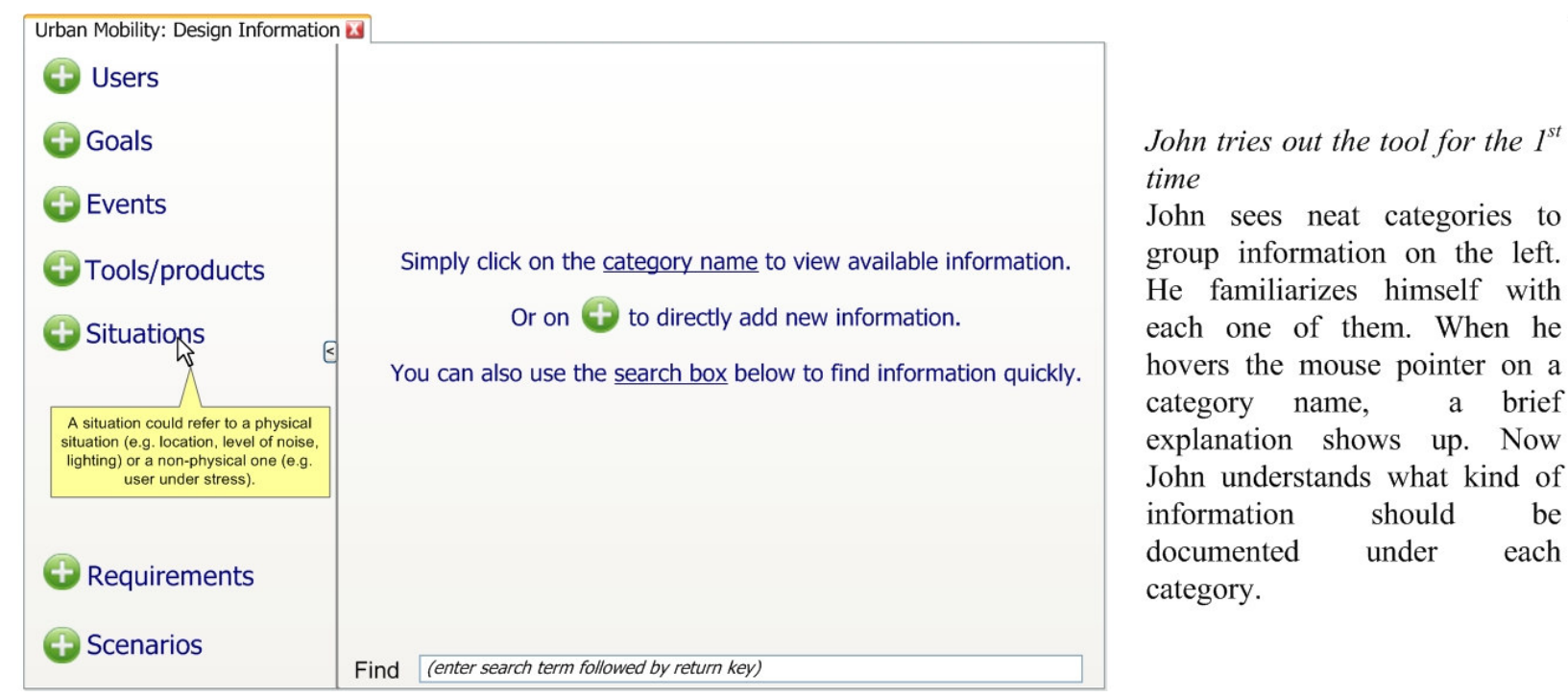

Figure 5.10: The welcome screen provides simple categories that guide the design team to document relevant information.

Following the introduction and first impression of the support tool, the main functions as proposed in this conceptual design are presented. These functions are directly related to the use case diagrams (Figure 5.2). To maintain clarity, each subsection will describe the Visio-based prototype and scenarios within their related use case.

\subsubsection{Use case: "Document design data"}

The tool provides a template to guide designers in classifying the design data into categories inspired by scenario elements. The existence of a template will provide an overview of how complete the design data is, and also inform design team members of the priority information. By making the information organized early on, the tool could sustain designers' motivation and enthusiasm while documenting throughout the project. Figure 5.11 to 5.14 show how the designers could register design data into different categories. The last one, Figure 5.14, gives a bit more insight on how the Visio prototype and scenarios are presented during the evaluation session; the interaction with the tool is shown step by step and animated using PowerPoint. 


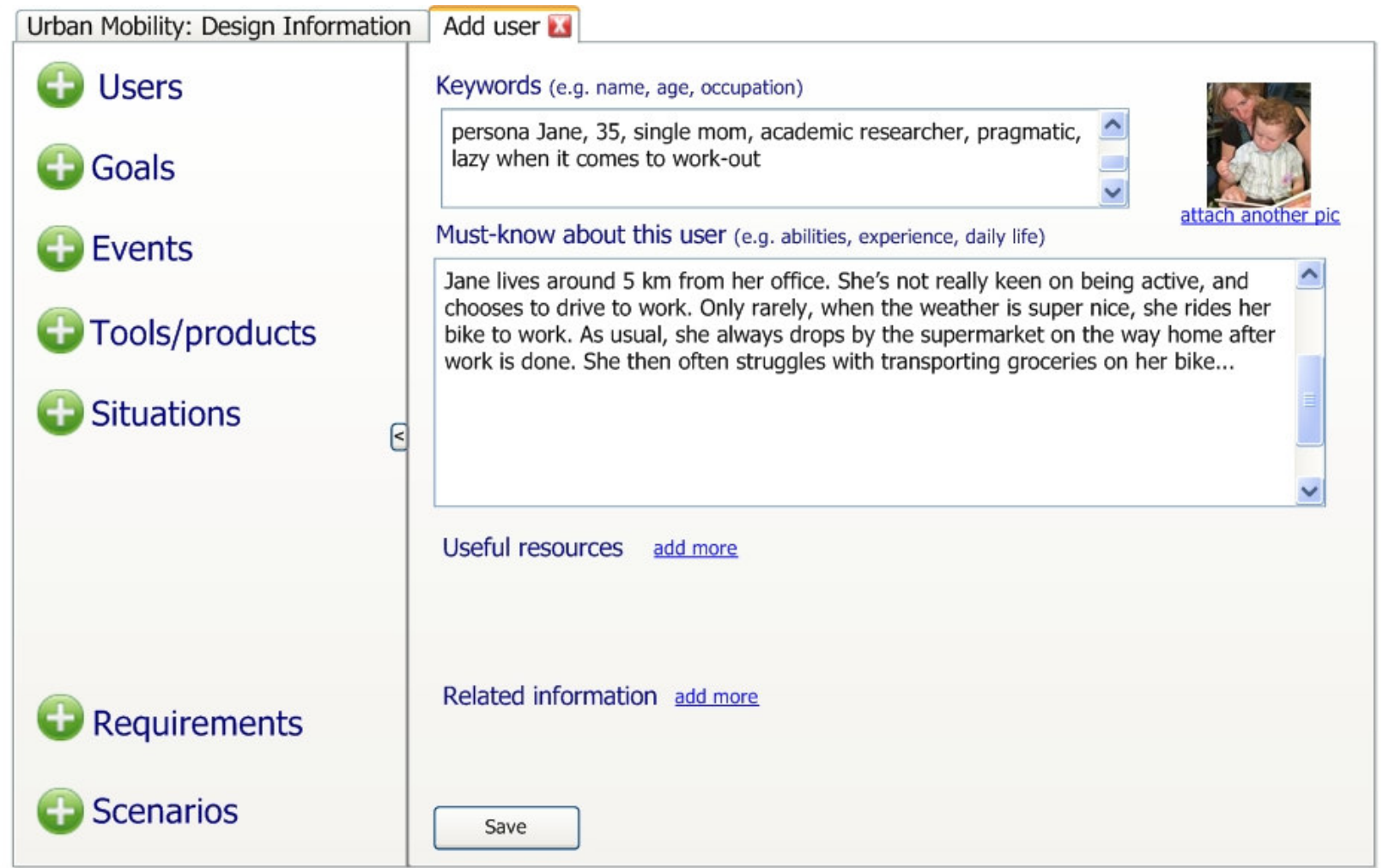

John creates a user profile using the scenario support tool

John summarizes what he has learned from the focus group session. Both participating users are quite similar in the way they are using bike pannier. Therefore John creates a fictive user Jane that combines their characteristics.

Figure 5.11: Adding an actor profile without much prescribed steps; the designer decides whatever information he or she feels necessary to include. 

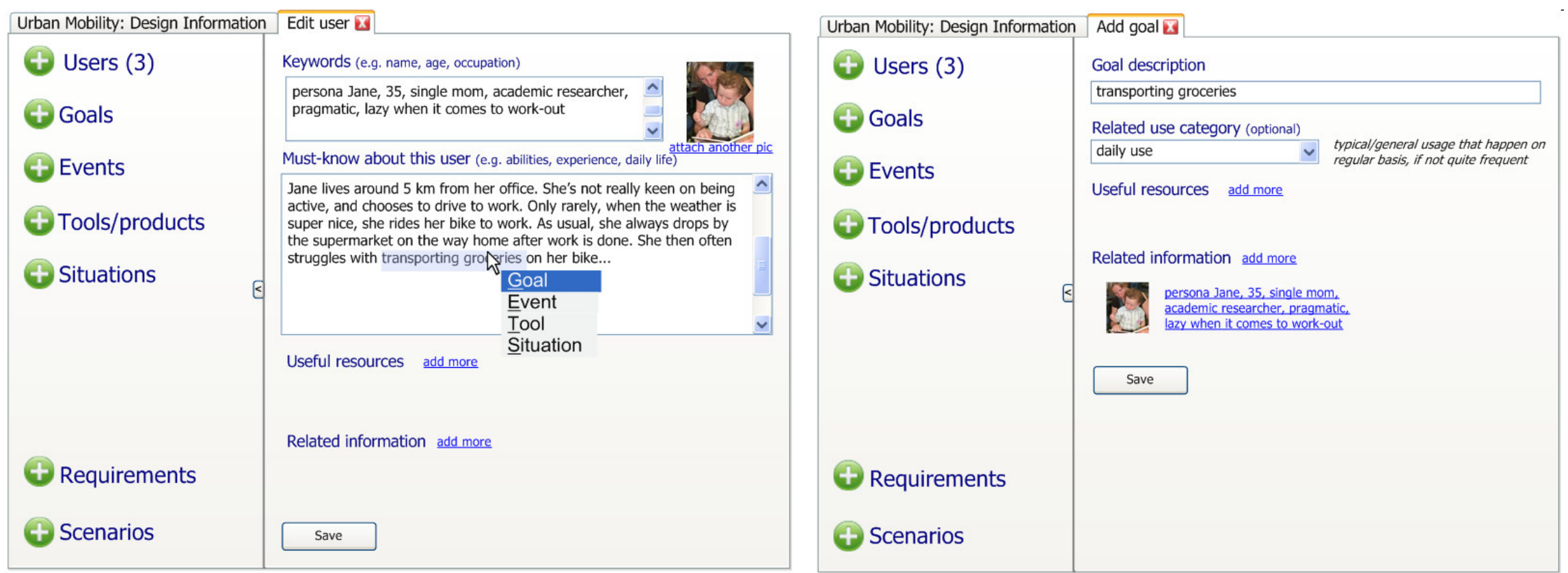

John identifies and extracts other scenario elements from a user profile

John revisits the user profile Jane and breaks it down further by scanning for other useful information. For instance, he notices a goal 'transporting groceries' is implicit in the user description. He marks the piece of text and by right-clicking, could register it as an explicit goal. The new goal keeps a reference to the user profile Jane as its source of origin.

Figure 5.12: An actor goal can be denoted from an actor profile; their relations are explicitly maintained for better traceability. 


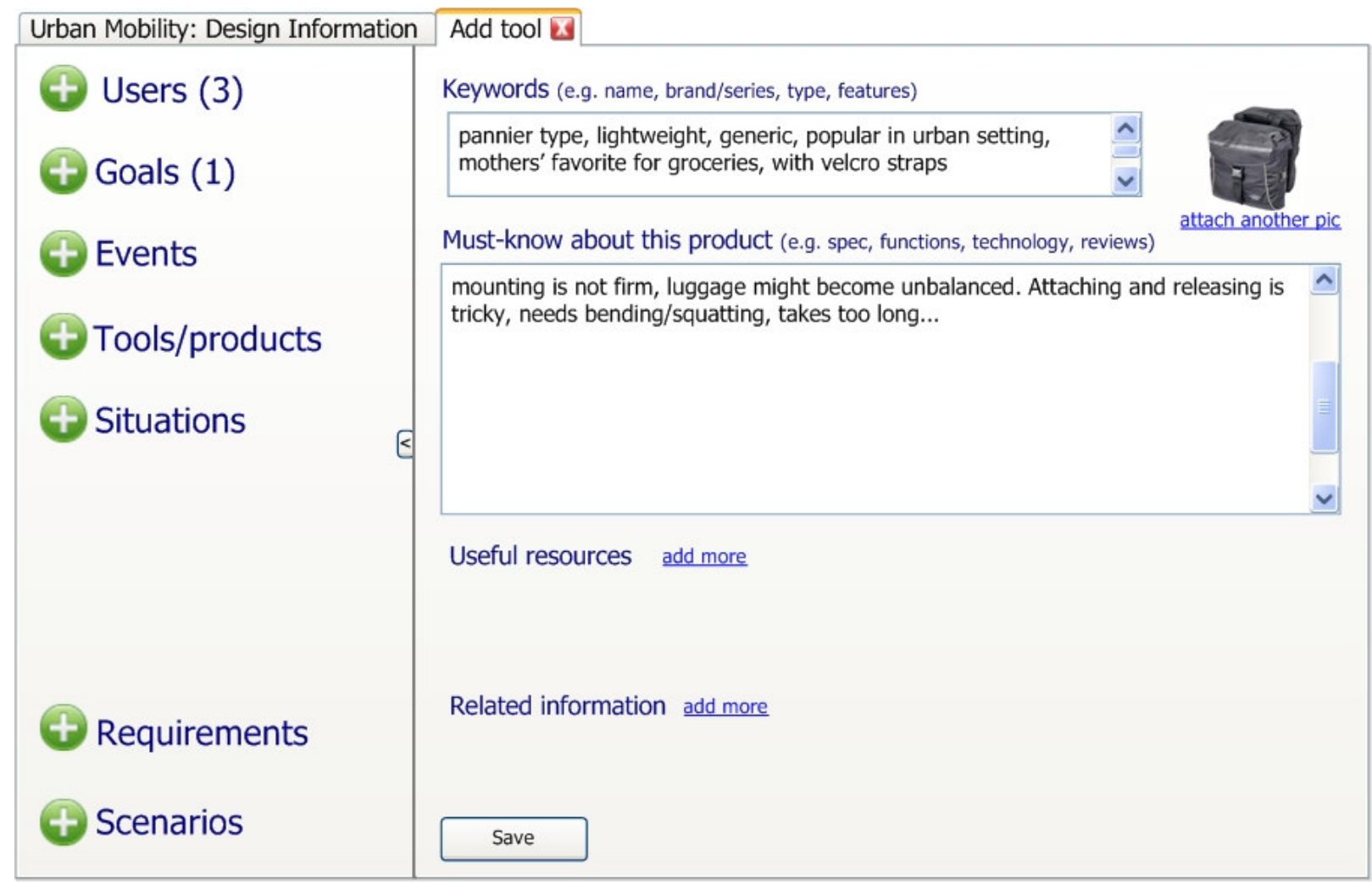

Marie adds product profiles and completes an existing product profile

Marie finished scanning the brochures from the fair and uploading pictures to the company's network drive. She adds new information in the 'Tools' category along with a summary of her notes. Based on her observation at the fair, she composes reviews on the pannier product. progress to make mention of its characteristics. 


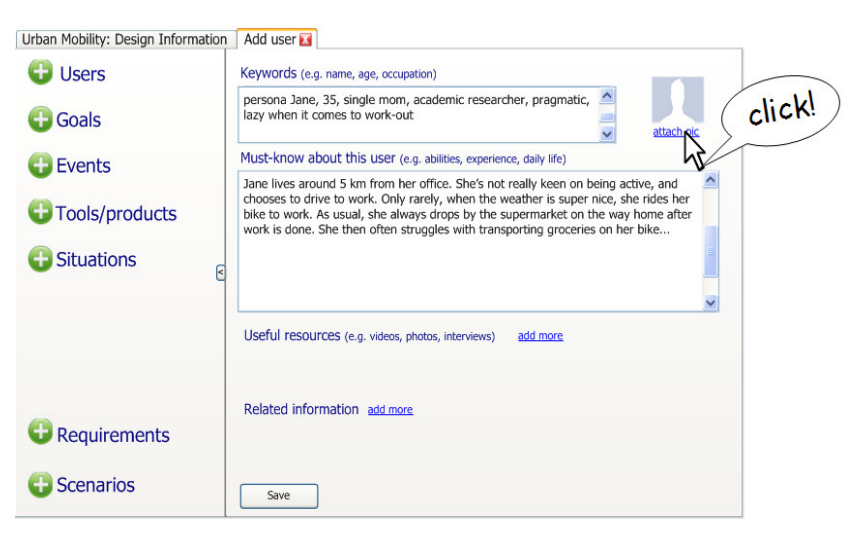

John wants to attach a picture, so that the user description can be quickly identified with a face.

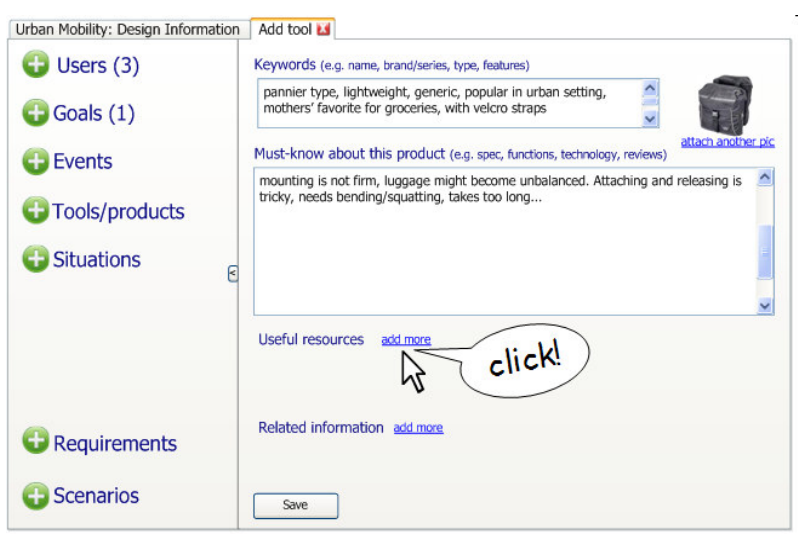

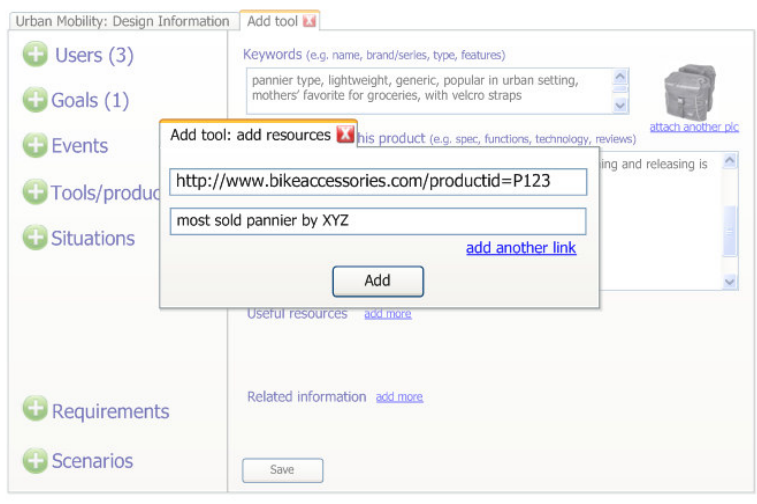

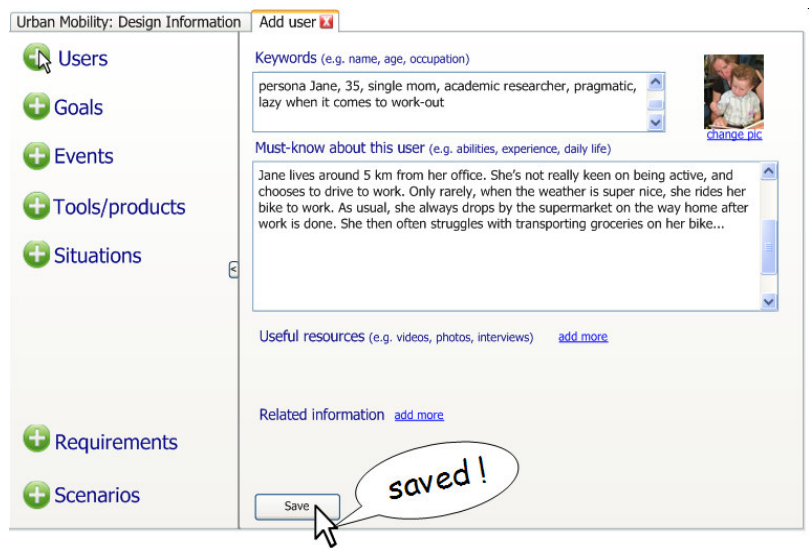

John attaches a picture to an actor profile.

He clicks on the blank picture. A small window appears, where John can select directory and picture file.

An initial profile of user Jane is created. Every team member can add more details to Jane's profile later.

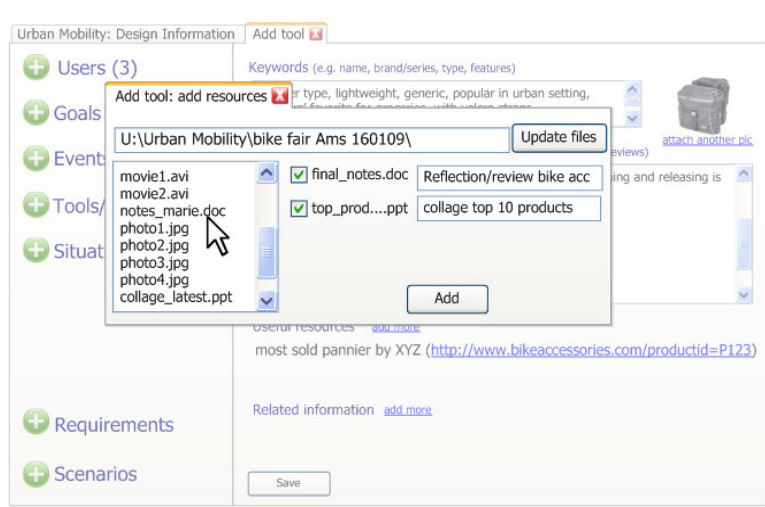

\section{Marie attaches useful resources to a product profile.}

Marie wants to add informative resources to the product profile. She clicks on the link 'add more' next to the 'Useful resources'.

She found earlier a link to online shop that shows detailed Marie attaches a few more internal documents to the features, price and user reviews of the product. She copies product profile. and pastes the link and adds a descriptive title. 


\subsubsection{Use cases: "Create scenario" and "Create requirement"}

In the next step, the documented design data could be transformed into scenarios that give a reasonable coverage of the product use. Scenarios narrow down the data into its essence. Furthermore, scenarios are vivid and therefore more memorable. The tool helps to identify and record combinations of data that could be the basis of scenarios (see illustration in Figure 5.15 and Figure 5.16). Figure 5.17 and Figure 5.18 illustrate the creation of tentative requirements by the designer, and the confirmation of these requirements by project manager. The idea is so that designers are not disrupted by having to formalize requirements in the middle of their creative process. However, this proposed function will depend on the practice of the organization (to be verified in the evaluation).

The tool also proposes a possibility to make explicit the relationships between different types of information. The relationships can be between the elemental information and scenarios, among scenarios, and between scenarios and requirements (as illustrated in Figure 5.18). This functionality is expected to improve the traceability of information. 


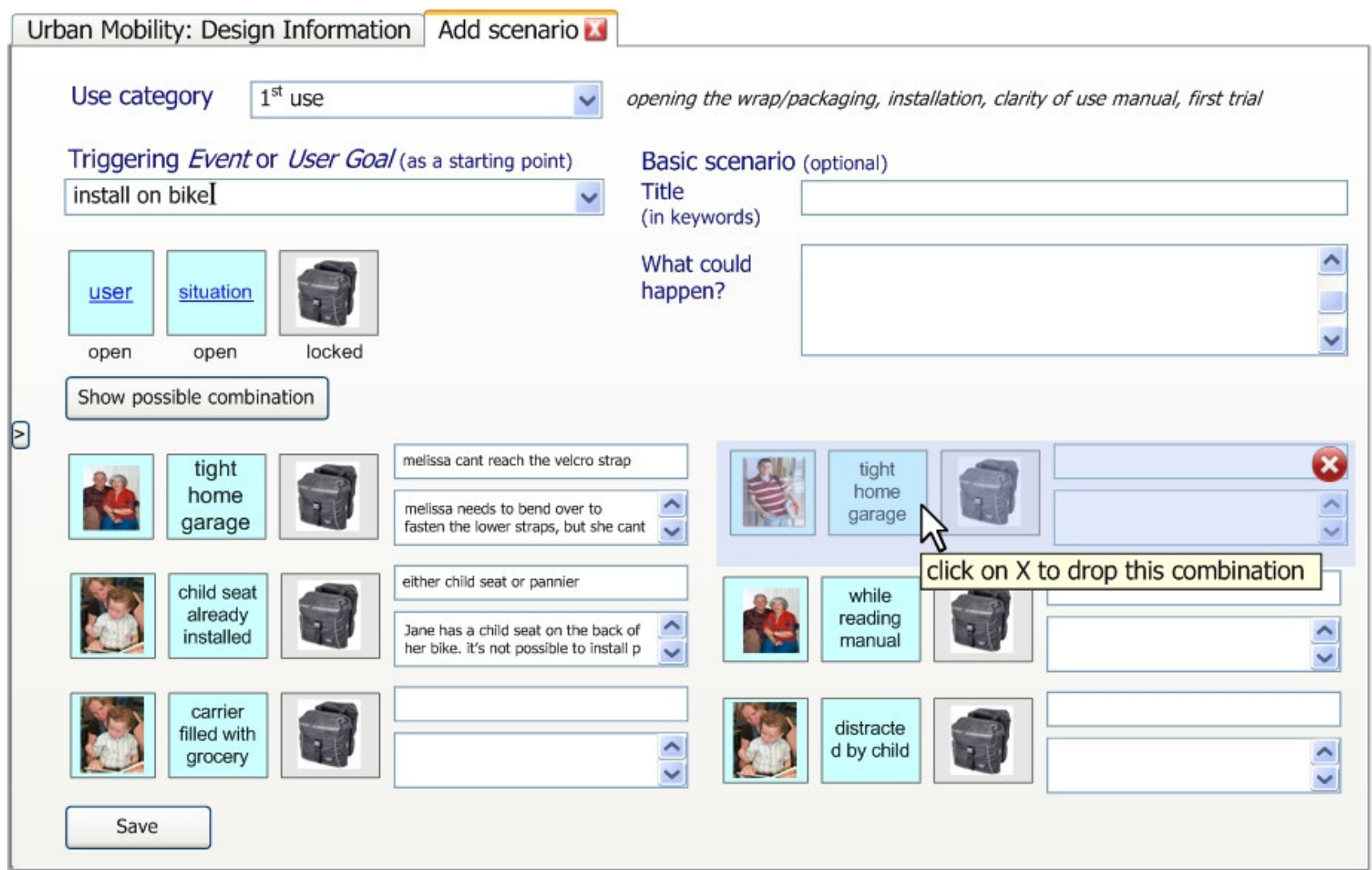

John generates scenarios

John starts identifying scenarios that contain important issues. He chooses a common user goal 'installation of product'. Then he pulls out other scenario elements: his main interest being the pannier product type. As John is not sure what other elements would make interesting scenarios, he asks the tool to show him possible combinations of existing information. A few of the combinations trigger John to scribble stories about what could happen. 


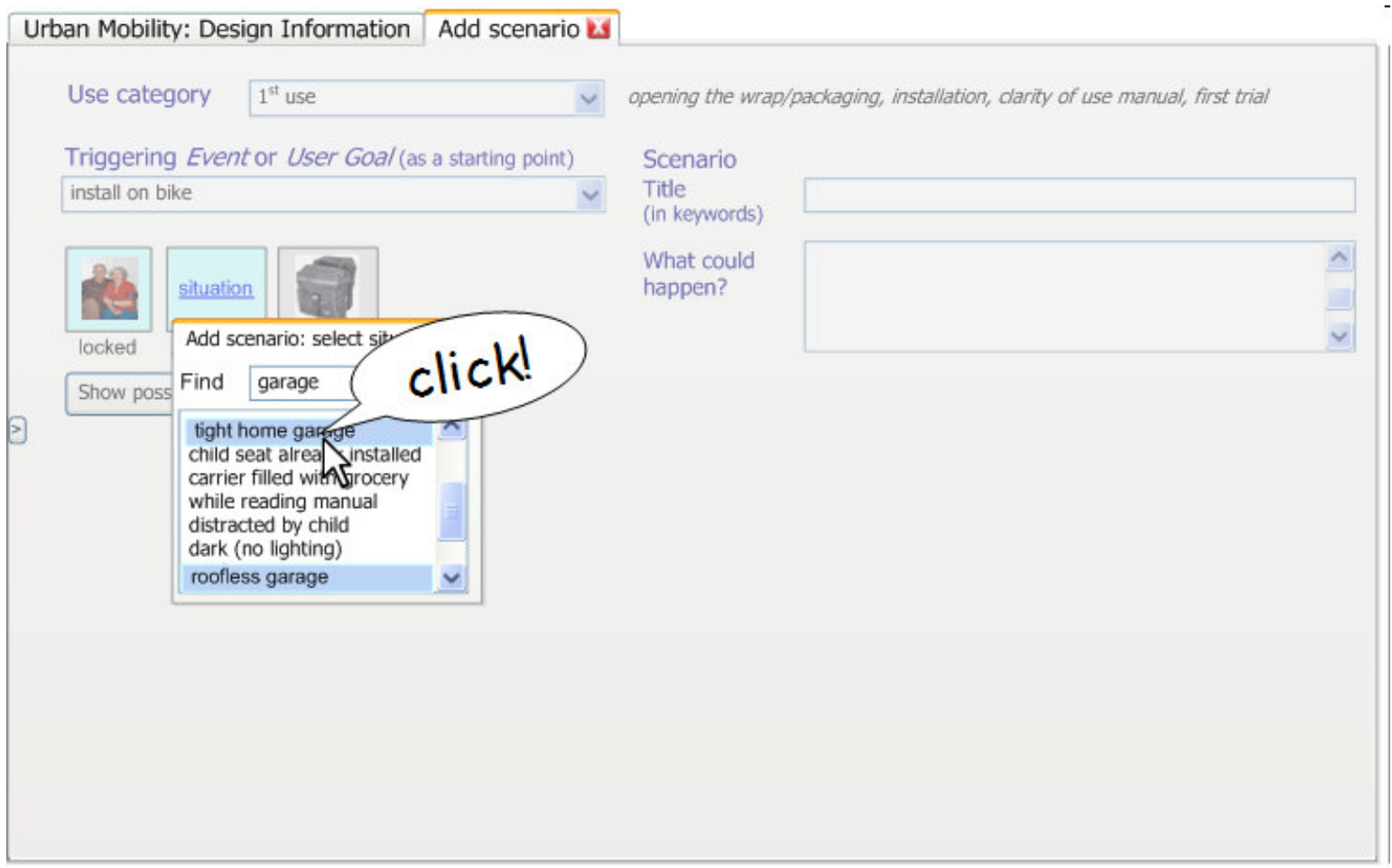

John wants to write a specific scenario based on his observation. He chooses the setting 'tight home garage'. As he fills in the elements...

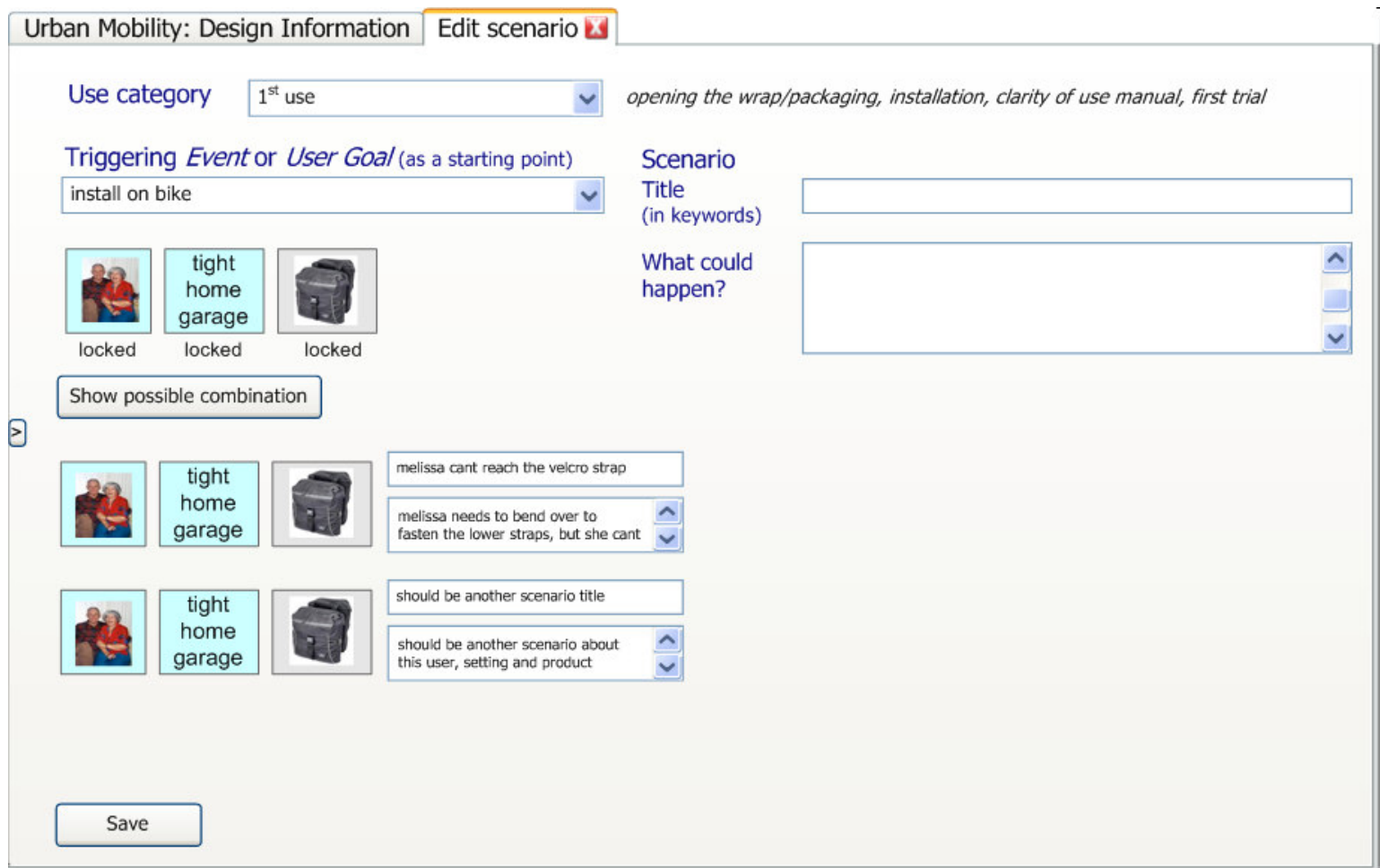

...he notices that some scenarios have been created. John can add or modify the scenario if he wishes.

Figure 5.16: The tool automatically displays a list of scenarios containing the specified elements. 

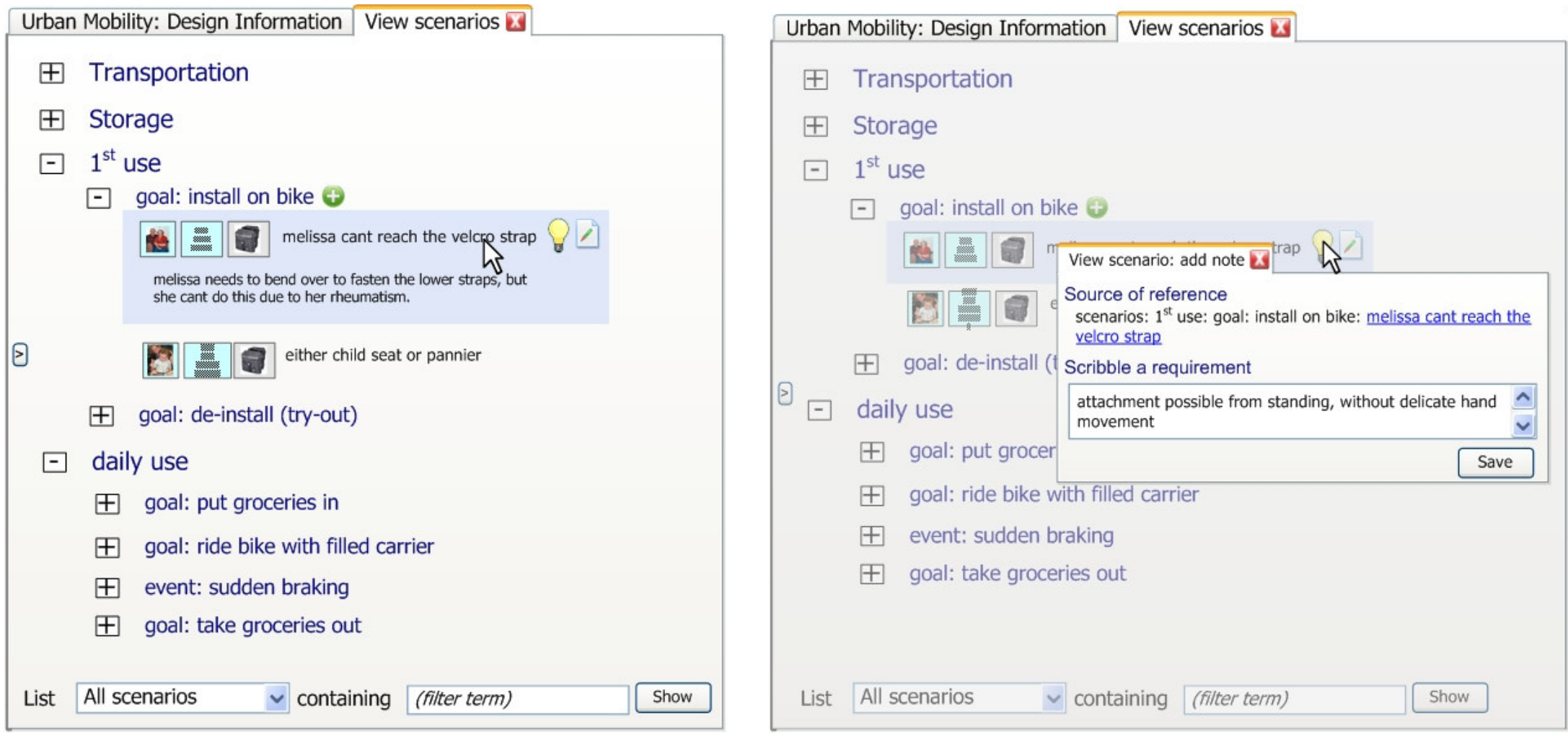

Marie scribbles requirements inspired by the scenarios

Marie scans through the scenarios related to 'installation of the product'. A scenario provokes her mind and she wants to immediately write down her thought. She clicks on the title to highlight the scenario. A set of icons appear next to the scenario title: the light bulb for note-taking and the pen \& paper for editing.
The tool keeps a record on which information has inspired this note, so that Marie can easily find it again for her own reference. After writing down her note, Marie continues scanning the scenarios for inspiration. She knows she can always come back to her scribbled notes later.

\section{Figure 5.17: A scenario could inspire product requirements; the tool accommodates the designer to readily make notes for tentative requirements.}



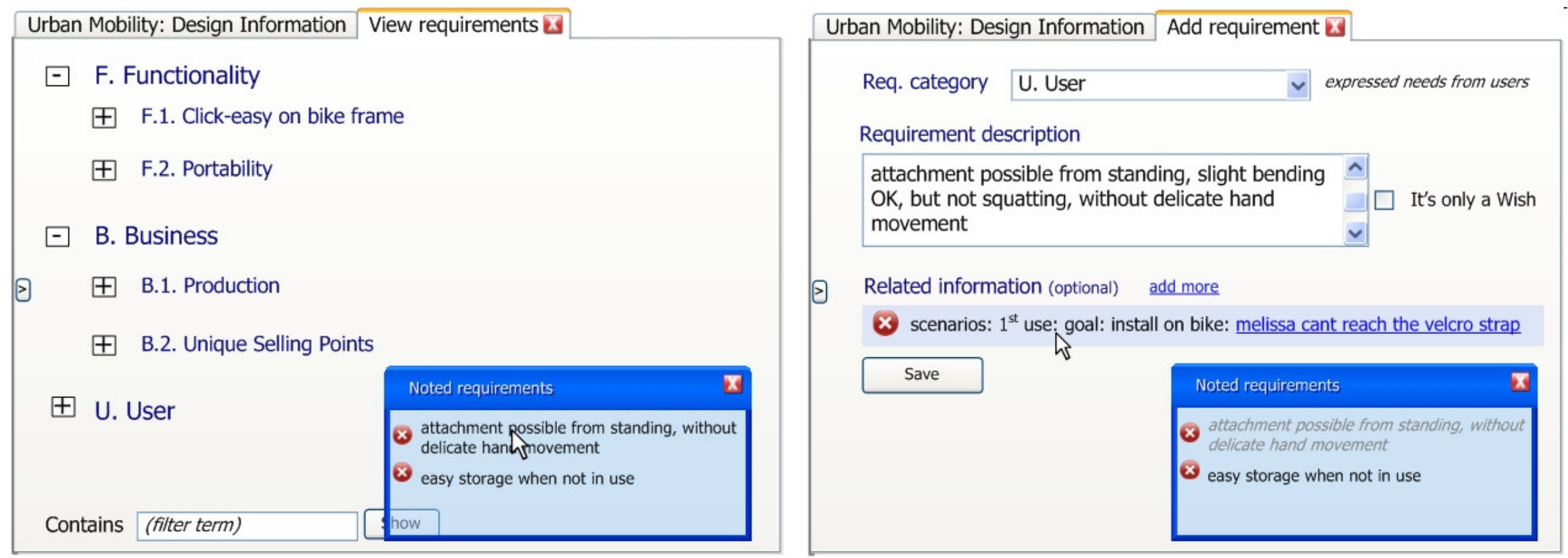

Bob registers a scribbled note as a formal requirement

Bob opens the requirements overview to check out how the team is doing with the analysis. He sees that the 'noted requirements' box now contains new tentative requirements. He clicks one of them about 'attachment (should be) possible from standing' to edit its details. He modifies the description and assigns a proper category for the new requirement, and then saves it. Bob notices that the scenario about Melissa that has inspired this requirement is saved as related info.

Figure 5.18: Tentative requirements can be promoted to formal requirements later (to be verified in the evaluation). 


\subsubsection{Use case: "Use information"}

The tool proposes an overview of scenarios in a process tree structure, which can be built according to the product life cycle. Such overview is expected to be easily understood by designers. There is a possibility to mark scenarios with specific keywords and priority ranks, to create semi-customized search results for individual design team members (see Figure 5.19). There are also different perspectives to view the information as there are multiple functions working together in a design team, as well as varied interests and purposes. The hierarchical structure makes it possible to display compact yet resourceful information.

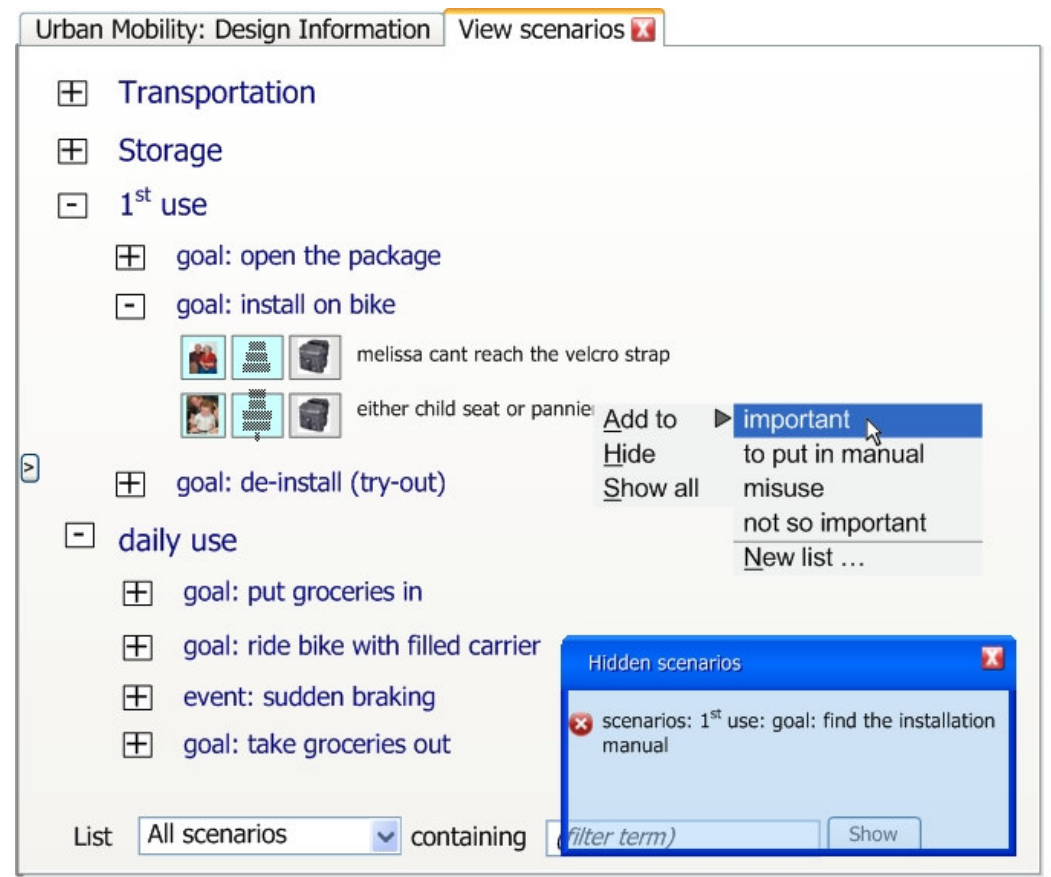

Marie wants to group scenarios on her own categories

Marie studies the scenarios overview. Scenarios about 'finding the installation manual' are not interesting for her; she already hid this scenario group. A small window keeps track of these hidden scenarios.

Marie adds a scenario to the 'important' list; the design team will have to pay special attention to it. Anyone in the design team can easily see the custom lists by choosing from the dropdown in the left-bottom corner.

Figure 5.19: Scenarios can be filtered and tagged; designers can select and prioritize scenarios according to their main tasks or interests either individually or a team.

The Visio prototype and scenarios are the means for evaluating the conceptual design with Indes designers, which have successfully stimulated reflection and feedback. The next section will describe findings, including the feedback received and ideas explored, from the focus group meeting.

\subsection{Findings}

Provided with the conceptual prototype, the designers could reflect thoroughly on the pros and cons of the proposed support tool; each argument sufficiently explained through their rationales which are based on their current practice at Indes. During the evaluation, two differing interests are recognized from the Indes designers and the researcher respectively. On the one hand, this research aims to deliver a tool that answers a more general practice of scenario-based product design. On the other hand, the designers wish for a tool that fits the very details of their practice. The feedback intermingles between these two interests, and among different levels of details. Although not all the feedback is relevant with this research, this section describes all the findings. However the next development steps of this research will remain focused on the main functionality, whose scope has been defined in the initial use case diagrams. To structure the findings, this section is divided into two main 
subsections, which address the verification of the functionality and other feedback respectively.

\subsubsection{Functionality Review}

The section addresses the feedback on the main functionality and other topics on the general level. The main functionality will be discussed using use case diagrams as a medium. This choice of medium is also practical since the suggested additions/changes in functionality can be easily reflected on the use case diagrams. Apart from the main functionality, other topics include discussions on the extra functionality that would improve the applicability of the support tool on a practical level. It is also occasionally too specific for the practice at Indes. The suggested extra functionality is outside the scope of this research - indicated as blue use cases in the figures. Therefore it is not included in the next steps of development, but will be addressed in this section since it is a part of the findings.

The main functionality has been verified as potential solutions to the problem areas in Indes (whose discovery has been explained in Chapter 4). The general use and interaction with the support tool, although still conceptual, can be assessed as acceptable in their daily practice. The strength of the support tool lies in its characteristics on being informal and creative. The tool should focus on inspiring designers to identify and write meaningful scenarios, without limits or constraints. The tool should not try to be extensive by covering all other formal deliveries, because then it would ruin its simplicity and making it less usable. However, to justify the efforts spent on using the tool, it is important that the tool is able to deliver more presentable information (see use case diagram in Figure 5.20). Depending on the format, it can be used e.g. for internal brainstorm meeting or for showing progress to the client. This information, although still informal, can also be useful for the creation of formal documents later on.

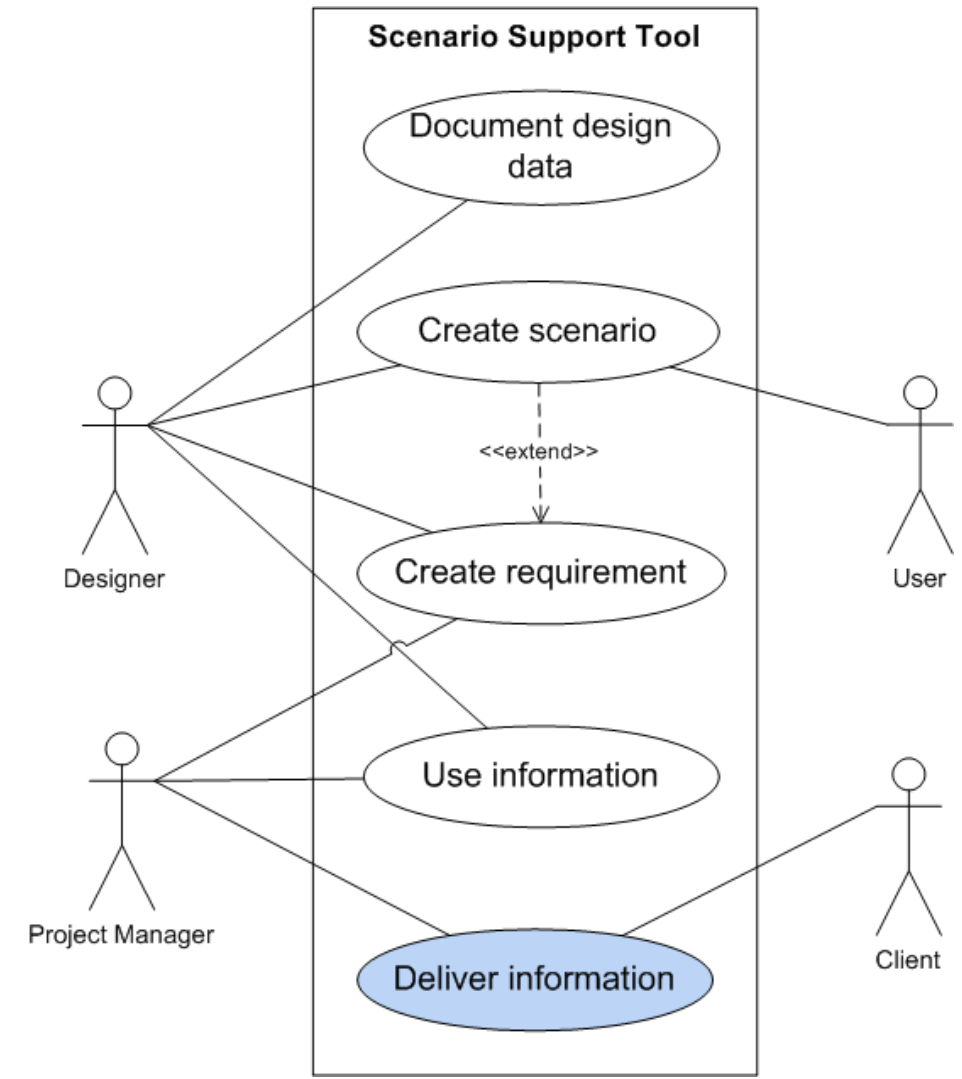

Figure 5.20: The support tool extended with functionality to deliver presentable information. 
In regard to the documentation of design data, the coverage/scope and structuring have been verified as sufficient. A small adjustment is to make it possible to register actor goal directly (see Figure 5.21). In the initial use case, actor goal should be registered via actor profile (see Figure 5.3). Additionally, it should also be possible to import design data, for instance from earlier design projects. The function to Import design data will be specific to a particular organization or project. Therefore while use case "import design data" is recognized in the use case diagram (Figure 5.21), this additional function will not be addressed in the development.

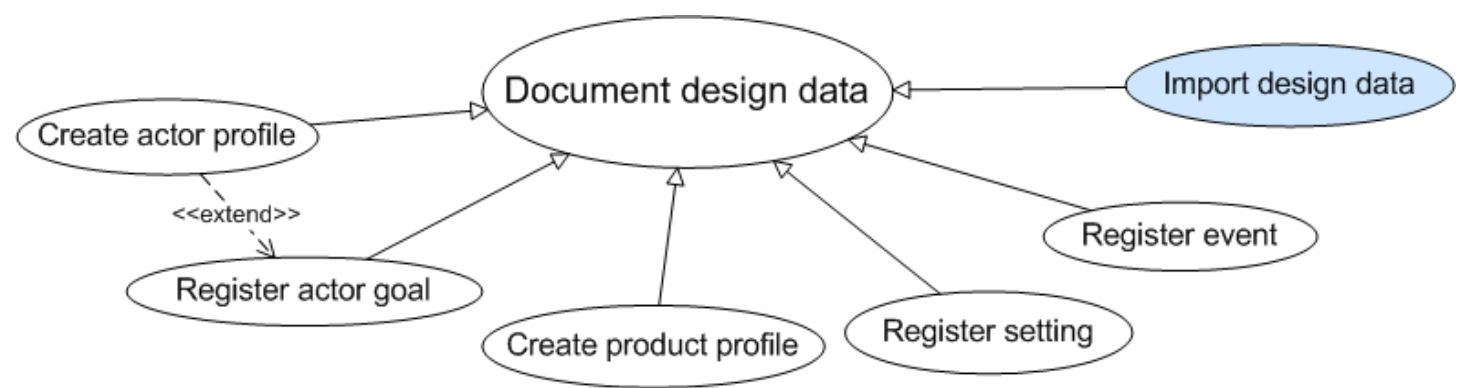

Figure 5.21: An new possibility to register actor goal directly and to import design data; the latter is considered outside the scope of the development.

To inspire designers during scenario creation, the tool can show random combinations of design data (i.e. scenario elements) to prompt designers of possible scenarios (Figure 5.15). The designers do usually get their inspiration in a similar way. For instance, a brainstorm session to identify critical scenarios could be inspired with printed cards of extreme actors (users), pictures of settings (locations) or misuse. This function is appreciated.

A scenario needs to be assigned a use phase when it is created. In practice, use phases (the tree structure in Figure 5.19 containing storage, first use, daily use, etc) are defined beforehand and can be used as a general guideline to identify scenarios. They provide a starting point to think about the situations that could happen in different phases of a product lifecycle. The steps for creating a scenario are verified as being logical. It is suggested that creating scenarios from the use phase overview should also be possible. This suggestion however is minor and therefore not considered as a part of the main functionality.

To make sure that the created scenarios are realistic and believable, the designers wish to be able to allow the users to confirm the scenarios. This wish will vary greatly between organizations or even between projects within an organization. Therefore, while use case "confirm scenario" is recognized in the use case diagram (Figure 5.22), this additional function will not be addressed in the development.

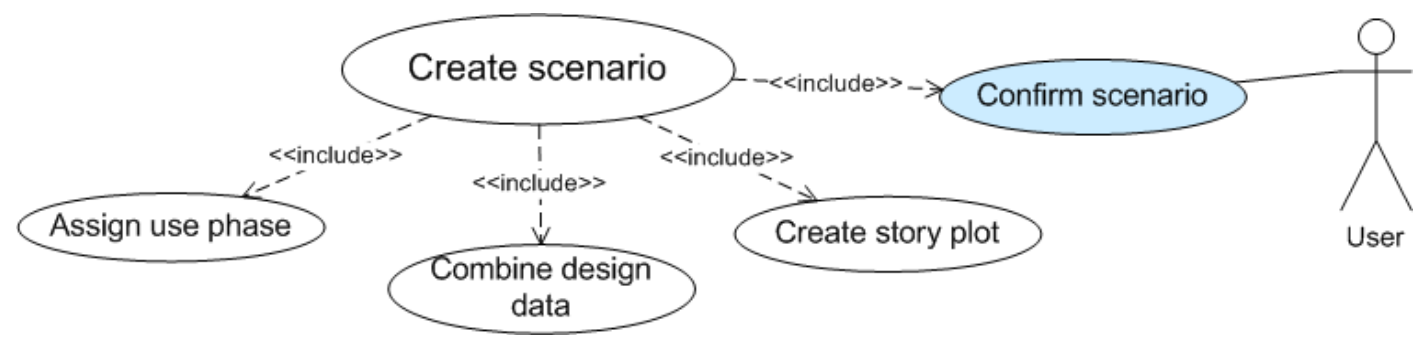

Figure 5.22: Steps to create scenario are confirmed; additional use case "confirm scenario" will depend on the specific practice or project. 
The initial use case "create requirement" involves two levels: the proposal and confirmation. A requirement is tentative by default, until it is confirmed (see use case Figure 5.5 and illustration Figure 5.17 and 5.18). This makes writing requirements less daunting to designers, by lifting off the pressure of having to deliver formal requirements at once. However, with the preference to keep the tool informal and non-bureaucratic, the designers wish to also simplify creating requirements by completely removing the confirmation step (see Figure 5.23). While there is a concern that simplified steps would cause duplicate requirements, it is not considered an important issue in the informal setting. For instance, if a 'requirement' is noted by several designers several times, it could be an indication that the requirement is very important. Taking into account their experience in conducting scenario-based design, this suggestion will be implemented although it is based on the practice at Indes.

Categories for requirements are important to keep information organized. However, these categories should be optional, as to not force designers to decide each time they create a new requirement. The categories should be as simple as possible (flat, no hierarchy) to conform to the informal nature of the support tool. The use of labels (tags) can actually be appropriate to group requirements together, since a requirement can be a part of several groups. It will be explored in the next development steps.

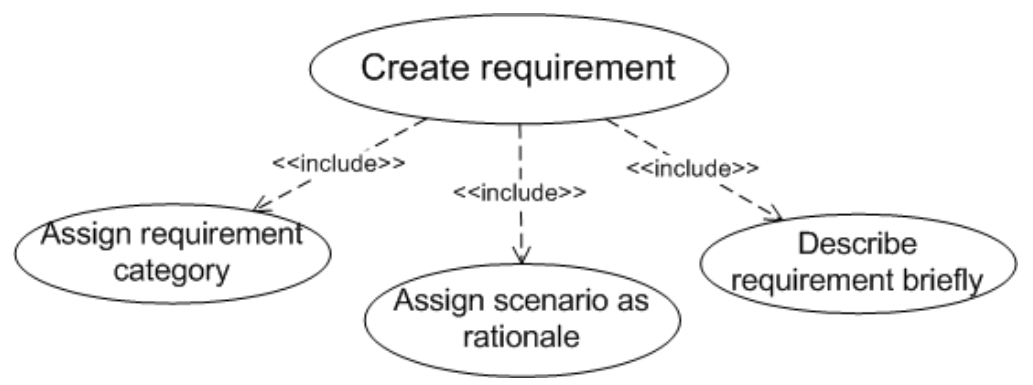

Figure 5.23: "Create requirement" is simplified into three direct steps.

The search function needs to cover all information, not only scenarios as originally proposed (see Figure 5.6). As the information grows, the designers wish to be able to use the tool also as a knowledge base of a particular design domain; thus the search function will be frequently used. Therefore a possibility to search and filter all different kinds of information is needed and will be an important part of the next development steps.

An additional function is proposed to display information in a more visual way. This function is considered outside the scope of this research. Therefore, while use case "display information" is recognized in the use case diagram (Figure 5.24), this additional function will not be addressed in the development. The designers however have come up with some ideas for displaying information, which are briefly addressed in the next subsection. 


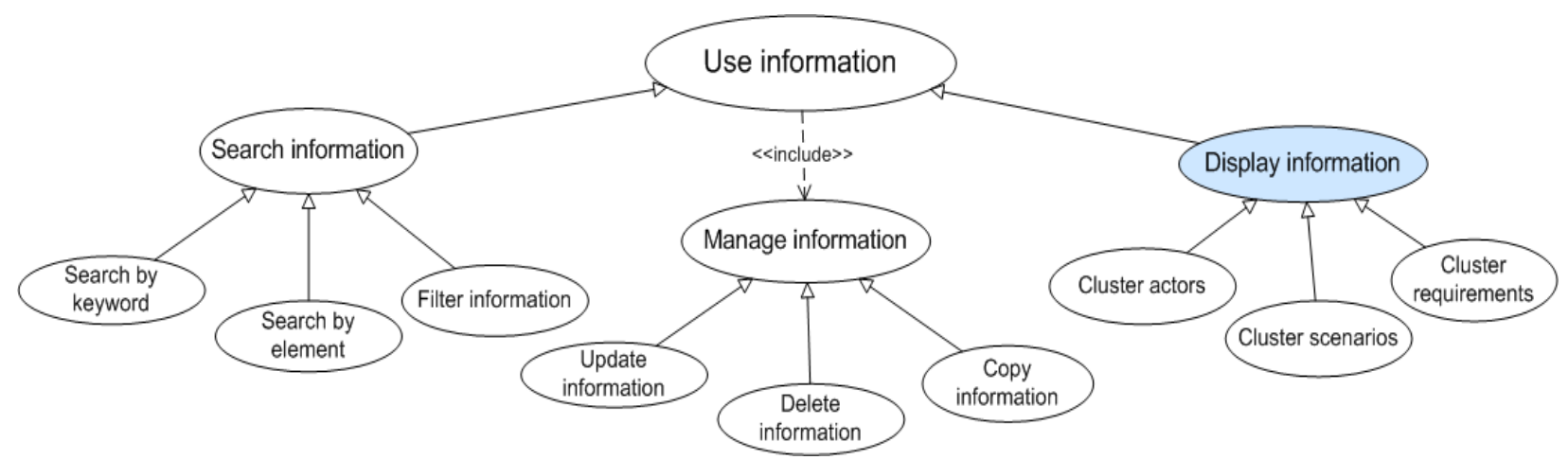

Figure 5.24: For a designer to "use information" will require a combination of the three functions, with "search information" being the most important.

Lastly, as shown in Figure 5.20, a completely new use case "deliver information" is proposed by the designers. This is a very practical requirement, and its implementation would depend greatly on the particular organization. Therefore, this additional use case will not be addressed in the development.

To briefly explain the motivation for this use case (Figure 5.25), the designers would occasionally need physical printouts that can be carried/annotated easily to e.g. informal meetings with colleagues, or formal ones with clients. The designers also expect that all the information will eventually contribute towards the final deliveries of a project. Therefore, a possibility to export information into specific formats is desired.

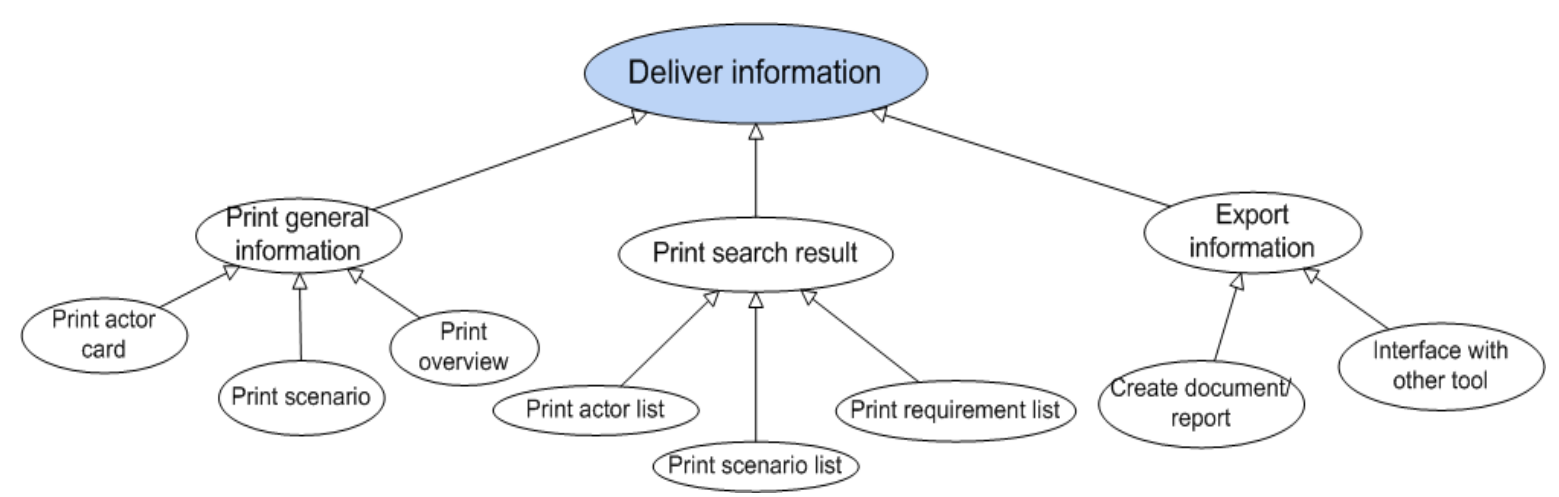

Figure 5.25: "Deliver information" concerns the practical outputs of the support tool; it is outside the scope of this research and will not be implemented.

\subsubsection{Other Feedback}

Some of the feedback concerns practice-oriented functionality - which is necessary for real implementation, but will not be addressed in this research. Nevertheless, as it is a part of the findings, the feedback is summarized in this subsection:

- The tool should define consistent input and output. The informal and creative tool which allows flexibility in documentation would work in practice if there is a good interface with other formal frameworks. With numerous formal frameworks and tools in design practice, this research draws the line here and does not attempt to address this topic. Discussing this topic, however, leads to other needs for the tool.

- The tool should track design decisions and contributions. As a means for collaboration, the tool needs to provide overview of "who has done what". While a detailed account of change history might not be necessary, it is important to be able to trace back every decision to "who and why". For instance, in the case a scenario is labelled as important or assigned as a risk, the tool must provide 
information on "who made the decision" and "for what reason". Data additions, changes and deletions also need to be recorded in the tool.

- The tool should use more visuals to represent information. Concerning the possibility to label scenarios to create custom-groups (Figure 5.19), the designers suggest a more visual alternative using 'flags' which can be colour-coded (like in Microsoft Outlook or Mindjet MindManager ${ }^{6}$ ). Additionally, some ideas are mentioned concerning a more effective visual overview of scenarios. For instance, a visual overview could contain cues to indicate the priority (or criticality) and relationships of the scenarios. To deal with a large amount of information, the overview should be adjustable in its scope, e.g. by allowing designers to see only small clusters of scenarios.

In addition to the feedback, the designers also identify future potentials of the tool. The open discussion leads to the identification of the web as an ideal platform for the support tool. The tool on a web platform would allow easier exchange of market research information between different agencies. For instance, project templates or personas or partial information concerning a typical design project could be used by other design companies. Such particular knowledge could guide inexperienced design teams in specific design cases.

Summarizing, the functionality as presented using the conceptual prototype has been reviewed and well received by Indes designers. While modifications have been proposed, the core functionality of the support tool has remained the same as initially defined. Other points of improvement have been discussed. However, since they are mainly practice-oriented - and therefore outside the scope of this research, they are not going to be addressed in the next development steps.

\subsection{Conclusion}

This chapter has described the conceptual design and its evaluation with the design practice. The main functionality of the support tool has been verified. Other ideas that would be required for real implementation of the tool in practice have been explored, including the identification of the web as an ideal platform for the tool. While they are not within the scope of this research, they will be recommended as a part of future work. In brief, by collaborating with the design practice at this conceptual phase, the evaluation has verified our view on the aspects of design activities, especially the process we aim to support.

The verified functionality will be the foundation of the next development steps. With the main functionality and the basic interaction sufficiently solid, the next step is to integrate them into a working prototype. A more interactive prototype will give a more accurate enactment of the interaction with the tool, and subsequently allow the designers to evaluate the implementation of their feedback. To build such prototype, we first need to investigate on the available (web) technologies that can be used. By doing so, this research is also more informed on what can be achieved, and how the evaluation should be planned accordingly. Chapter 6 elaborates the process to define an evaluation setup and the investigation of available technologies for the prototype development.

${ }^{6}$ A tool to create visual information maps (mind maps): http://www.mindjet.com/. Retrieved on Aug 30, 2010. 



\section{Part III \\ Implementation and Evaluation}





\section{Evaluation Setup and Implementation}

As this research aims for a user-centred design process, multiple evaluation phases with a focus on usability are anticipated. To serve this purpose, an interactive prototype is needed to capture and demonstrate the functionality and interaction as proposed in the conceptual design (Chapter 5). As the web has been identified as an ideal platform for the tool, the prototype is expected to be implemented as a web-based application. It is important to mention that the prototype will not be evaluated as a complete software package, but rather as a means to implement our ideas about scenario-based design.

Based on the definition of usability, e.g. as a software quality according to ISO/IEC 9126-1 (ISO/IEC, 2001) and as the overall goal that the software meets user needs (Bevan, 1999), a broader objective of usability is adopted for the evaluation of the tool. Usability is the capability of the product to be understood, learned, used and attractive to the user, when used under specified conditions. In terms of quality in use, usability is the capability of the product to enable specified users to achieve goals with effectiveness, productivity and satisfaction in specified contexts of use. Usability will be measured as the combined effect of the categories. From this perspective, this research anticipates three distinct levels of evaluation. Each level is aimed at providing the researcher with specific feedback which will be incorporated in the development.

\subsection{Evaluation Criteria}

Among the categories that make up the quality of use, we focus on evaluating the effectiveness, productivity and satisfaction as the categories most relevant to this research. A reinterpretation of usability is therefore composed to structure the evaluation process.

\section{Effectiveness focuses on functionality}

The first important step is to verify whether the tool offers the functionality that is needed by the users. This has been partially done earlier during the requirements gathering (Chapter 4 ) and conceptual design (Chapter 5). With the dynamics of the development process, it is necessary to conduct a continuous verification of our requirements-to-functionality translation with the users. Consequently this evaluation phase demands a clear view of the future use of the support tool. Chapter 3 and 4 describe the methods used to get early feedback by using scenarios and mock-ups. With a working prototype actualized, the next evaluation steps focus on the functions and information flow of the tool. By the facilitation of workshops, focus group meetings, etc, the users are expected to be able to give more concrete feedback whether the implemented functionality makes sense in practice. At the same time, this approach also gives insight on the users' acceptance towards the support tool.

\section{Productivity (efficiency) focuses on a proficient interaction}

Once the functionality is confirmed by the users, the next step is to assess whether they find the interaction with the tool comfortable. This could be translated into many more detailed qualities, such as how easy it is to perform a specific goal, how easily accessible the stored data is, how informative is the tool and so on. All boils down to one question: how intuitive is the interface? This evaluation phase deals with the day-to-day (frequent) interaction of the users with the tool. Though the 
interface and appearance are not the sole focus of the development, the quality of the interaction depends greatly on them. The aim of this evaluation is to get an indication of the form of interactions enjoyed by the target users. This is planned by conducting focus group meetings, participatory design, and informal discussions that generate direct open feedback. From this feedback, the development process aims to implement the preferred interactions using the available interfaces and technologies.

3. Satisfaction focuses on the overall acceptance from the users (i.e. designers)

Users generally feel satisfied when a product answers their personal goals. While satisfaction is largely emotional, the fulfilment of the previous two levels is a prerequisite. There are many factors that come into play to the combined effect of satisfaction. These factors may differ between persons, even more between persons working in different environments or organizations. The rule of thumb is to fit the tool as natural as possible with the preferred way of working and thinking. On top of this, the tool needs to possess clear added values, for instance by aiding tasks which the users find challenging. With so much cognitive actions involved, in-depth studies with select design practices have been conducted (Chapter 4). Our requirements have been extracted from learning the practices of a limited number of design companies. Therefore we also wish to assess the tool in specific real-life scenarios in other settings or organizations. Although it is not possible to design an all-fitting support tool, feedback from a variety of designers gives insights into the characteristics of design practices or projects that could benefit from our tool.

To ensure quality feedback from the evaluation, a number of aspects are identified and taken into consideration in the planning. With a focus on user-centred design, the involvement of the target users as testers is the most important aspect. Furthermore, the evaluation should also be informed of the various design practices that are represented by these target users. By knowing more about the contexts, we will be able to compare the perspectives of the different practices regarding the support tool. Other considerations are the means (media and technique) to actually conduct the evaluation, as well as the strategy to develop the prototypes. The next sections will describe these evaluation aspects in more detail.

\subsection{Target User}

The most important aspect of the evaluation is the target users. The evaluation must involve a variety of users which the tool aims to benefit. This means diverse designers, with different levels of experience, varied functions and expertise. Additionally, a variety in the work practice/organization culture that shapes the context is also considered. The variety of target users during the evaluation should represent non-biased, objective and unsuspecting future users of our support tool. Their involvement will inform this research the work characteristics/contexts in which the support tool will bring added values.

Throughout this research project, contacts have been built with various design practitioners, especially the ones with a focus on usability and experiences with scenario-based design. The requirements capture and conceptual development have involved design practitioners with experiences in conducting scenario-based design projects - the larger part is the designers at Indes. As a small/medium sized design agency, Indes possesses characteristics that allow efficient collaboration with their designers (Chapter 4). Their insights and knowledge in the field has informed the direction of the support tool. However, in practice not all designers are familiar with creating, using and managing scenarios in their projects. To make sure that the support 
tool is also accessible to the less experienced designers, the evaluation will involve them and use their feedback to improve the tool (see Chapter 7). Additionally, to check whether the support tool adds values to the industry at large, the evaluation also anticipates involvement of another company with different characteristics from Indes, i.e. Philips as a representative of the "big company" culture. The main difference between Indes and Philips lies in their organizational structure and the work practice. In brief, Indes focuses on working in small yet dynamic teams of all-round designers who are familiar with the whole process of design. On the other hand, Philips focuses on assigning responsibilities to specific roles in a development team, which specialize in delivering their own responsibilities. The final evaluation (Chapter 9) will involve design practitioners from both Indes and Philips.

\subsection{Means of Evaluation}

A number of prototypes are developed in different levels of fidelity. In the early stage, the prototypes were largely in the form of paper sketches. Later they were refined into digital version (Visio drawings), with Powerpoint animations to simulate a supposed interaction with the tool. Scenarios were created to accompany these rough concepts, to make explicit their imagined use. In the next development stages, the aim is to translate the concepts into more interactive and dynamic prototypes. As identified in the conceptual evaluation (Chapter 5), the web platform is considered ideal for the support tool. Therefore, the final prototype is expected to be a web-based application implementing the core functionality. In each stage, the evaluation method depends on the media and the level of fidelity of the prototypes.

With the early prototypes, the evaluation method was more informal and the expected feedback more open. Many directions have been explored and new ideas from the users considered for improvement. The iterative evaluations conducted with designers employ a number of design methods, e.g. interviews, workshops, focus groups and questionnaires. Chapter 4 and 5 have described the methods used to involve stakeholders in the research. While earlier methods have been conducted heuristically, a more rigorous method to validate the final prototype is anticipated. Development milestones have been set for a mid-evaluation with our target users, as presented in Table 6.1. 
Table 6.1: The evaluation plan of the support tool.

\begin{tabular}{|c|c|c|c|}
\hline & Aim of Evaluation & Media/tool & Method \\
\hline$\frac{\frac{n}{d}}{\frac{\frac{1}{0}}{0}}$ & $\begin{array}{l}\text { - Functionality } \\
\text { - Fittingness in work } \\
\text { process } \\
\text { - Information flow } \\
\text { - Basic interaction }\end{array}$ & $\begin{array}{l}\text { Paper prototyping } \\
\text { translated to Visio } \\
\text { drawings, } \\
\text { animated with } \\
\text { Powerpoint }\end{array}$ & $\begin{array}{l}\text { Focus group with product designers } \\
\text { Future use scenarios, enacted using } \\
\text { Visio drawings and Powerpoint } \\
\text { animations } \\
\text { Discussion and qualitative feedback }\end{array}$ \\
\hline$\frac{\frac{1}{d}}{\frac{1}{0}}$ & $\begin{array}{l}\text { - Verification } \\
\text { implemented } \\
\text { functionality } \\
\text { - Interaction }\end{array}$ & $\begin{array}{l}\text { Web-based } \\
\text { prototype } \\
\text { (intermediate } \\
\text { version) } \\
\text { Mockup data } \\
\text { using fictive design } \\
\text { case }\end{array}$ & $\begin{array}{l}\text { Focus group with product designers } \\
\text { Walk-through all implemented } \\
\text { functionality with the designers } \\
\text { Discussion and qualitative feedback, } \\
\text { questionnaire }\end{array}$ \\
\hline $\begin{array}{l}a \\
\infty \\
\infty \\
\frac{1}{d} \\
\frac{\alpha}{0}\end{array}$ & $\begin{array}{l}\text { - Overall interaction } \\
\text { - Efficacy in varied } \\
\text { work practices }\end{array}$ & $\begin{array}{l}\text { Web-based } \\
\text { prototype (final } \\
\text { version) } \\
\text { Mockup data } \\
\text { using fictive design } \\
\text { case }\end{array}$ & $\begin{array}{l}\text { Focus group with product designers } \\
\text { from different practices/companies } \\
\text { Walk-through using future use } \\
\text { scenarios } \\
\text { Discussion and qualitative feedback, } \\
\text { questionnaire }\end{array}$ \\
\hline $\bar{U}$ & $\begin{array}{l}\text { Overall usability in a } \\
\text { design project, } \\
\text { including intuitiveness }\end{array}$ & $\begin{array}{l}\text { Web-based } \\
\text { prototype (final } \\
\text { version) }\end{array}$ & $\begin{array}{l}\text { Multiple-day use trial by designers in } \\
\text { one company } \\
\text { Reflection together with the designers }\end{array}$ \\
\hline
\end{tabular}

To be able to evaluate the categories as laid out in Table 6.1, the prototypes need to serve specific goals. Firstly, the prototypes must accommodate the implementation of the functionality as defined and refined in Chapter 4 and Chapter 5. This includes documenting and retrieving design data, creating scenarios and requirements, and organizing the use of information. Secondly, it needs to make explicit the process or work flow of creating and managing scenarios using the tool. During the later phases of evaluation, in which the designers get to try the tool without assistance, the interface needs to be clear and intuitive. Thirdly, the prototype needs to portray the future interaction with the tool so that the designers (testers) could reflect on and give feedback according to it. The interaction with the prototype needs to be as realistic as possible to make sure that the designers' acceptance is not misinterpreted. The following section will describe the implementation process of the prototypes needed in the next evaluation phases.

\subsection{Prototype Implementation}

With time and resource constraints, this research dedicated some effort to choose the right technologies to implement the prototypes. The implementation phases are described in the following subsections.

\subsubsection{Choice of Technologies}

There is a trend that software applications are moving towards web platforms. Part of the reasons is because applications on web platform are easily accessible -the user only needs a compatible web browser on his or her computer to run such applications. Furthermore, the installation of a web application and its updates can be performed on 
the server, therefore saving time compared to the possible complications of having to install and adjust the application on different configurations. This feature also accommodates growth in the functionality and the amount of data, making a web application more scalable compared to a desktop one for instance. Taking this trend into consideration, we therefore chose to focus on technologies for web application development. Among the available technologies, we lean towards the open-source over the dedicated ones. The availability of support from the open-source community means more possibilities to extend the technologies. Therefore open-source technologies are chosen to implement the support tool.

After narrowing down the focus on open-source technologies, the next requirement is to find a combination of technologies that is easy to use to demonstrate the workflow and interaction of the support tool. Among the capable technologies, some can be overkill and too demanding for the available resources within this research project. An example of such technologies is the Apache MyFace, an open-source implementation of Java Server Faces. Though it has a strong community support and extensive libraries to make a fully working application, it also demands an all-round technical expertise, which was not available within this project.

Recently the web also noticed the rise of technologies for Content Management Systems (CMS). A Content Management System allows a group of people to contribute to stored data, aids easy storage and retrieval of data, and regulates their access to the data. Contents are made up of data, which can be defined as almost anything documents, movies, pictures, phone numbers, scientific data, etc. The contents are industry-specific depending on the application domain of the CMS. With the trend of content-sharing over the internet, there is a growing number of web-based CMSes. A Web CMS basically facilitates content creation, control, editing, and other essential Web maintenance functions. There exist Web CMSes implementations in various programming technologies. Many of them are built on top of separate content management frameworks (CMF). A CMF provides a user-friendly programming interface for creating a customized CMS. Imagine a CMF as a toolbox with a basic set of generic interfaces, which allows the developer to configure and customize his or her own specific contents. Figure 6.1 illustrates the structure of CMF and CMS, and their relationships with the actors, i.e. users and developers in the community.

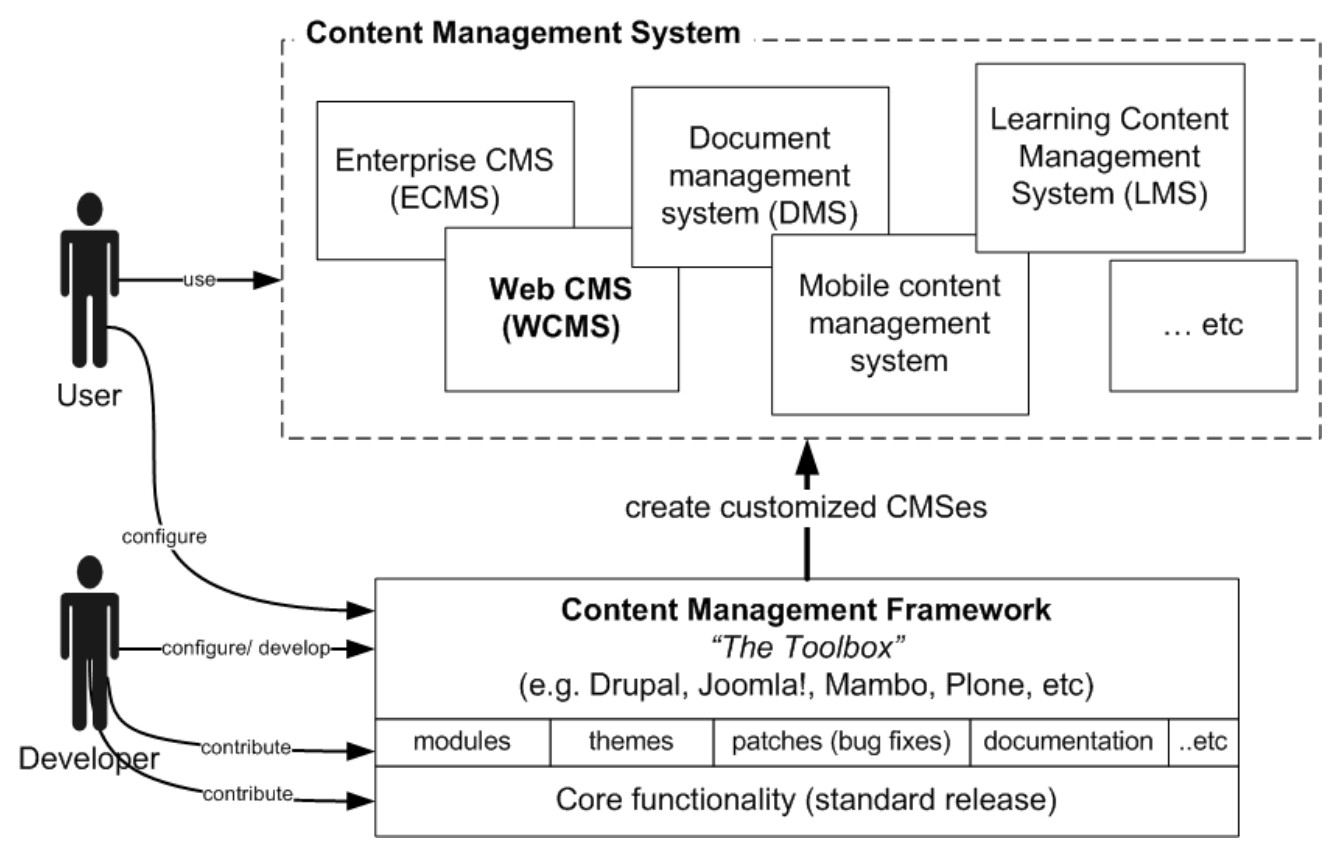

Figure 6.1: The organic structure of open source CMFs and CMSes. 
CMFs seem to fulfill the requirements as well as the constraint in this research. Two major CMFs that are applicable to this research have been surveyed: Drupal (Drupal, 2010a) and Joomla (Joomla, 2010). Both are built using PHP, a widely used scripting language for dynamic web development. Due to time constraint, the comparison of the two CMFs was performed heuristically based on the core functionality, extendable features, and ease of configuration and administration. Burge (2010) has compiled a comprehensive comparison of Drupal and Joomla. While neither one is better than the other, each one is better for different purposes. Essentially, Joomla wins over its userfriendliness and an active developer and designer community. Drupal, on the other hand, is more flexible and developer-friendly with a more coherent and stable community (Burge, 2010).

The implementation phase aims is to build a functional prototype of the support tool. With a flexibility to modify and add custom functions, Drupal is chosen as the development platform. Additionally Drupal offers a strong feature, the hierarchical taxonomy system, which deserves a specific mention. The taxonomy system allows content to be categorized or tagged with keywords for easier access. This feature has the utmost relevance to answer the required support for managing design information. Since Drupal is prebuilt, there is potentially a limitation in actualizing the specific interfaces and interactions as proposed in the conceptual design (Chapter 5). However, the prototype will be developed mainly to demonstrate the approach in using the support tool. Therefore the interfaces and interactions made available by Drupal are considered sufficient to realize the intended tool functionality. The following section explains more about Drupal and details its characteristics that are beneficial for the prototype development.

\subsubsection{Drupal as a Prototype Development Platform}

Drupal is a free open-source Content Management Framework, written in PHP. Any platform that supports a web server capable of running PHP and a database could run Drupal. Due to its versatile quality, Drupal is used as a back-end system for a variety of websites, ranging from personal blogs to enterprise collaboration and knowledge management for large corporate and political sites (Lincoln, 2008). No programming skill is required for basic website installation and administration, making Drupal popular among a large and active community of users and developers.

The standard release is the Drupal Core, which contains basic features common to CMS. The features cover for instance, interrelated systems for content definition, user management, permission, menu, simple web layouting, logging, and administration. On top of the core, there is an extensive list of modules contributed by the Drupal community. These contributed modules offer additional features and custom behaviours to the standard Drupal. Altogether Drupal and its supporting modules are capable of building different types of CMS for specific industry.

Our consideration has been to conduct rapid-prototyping using a configurable CMS with enough functionality and structure. To elaborate our reasons for choosing Drupal, some characteristics of Drupal have been acknowledged and summarized as the following (Geller, 2008):

- A "middle-ground" between customizability and "out-of-the-box" ease. While it is useful to know some web/programming skills (HTML, PHP, SQL), what is necessary to know to install and customize a Drupal website is not much and well-documented in the handbook (Drupal, 2010b). With time and resource constraint in this research, Drupal offers just the right balance between functionality and ease of use for building web prototypes. 
- Proven and secure. Drupal has been around for several years and used by thousands of sites, some of which are mentioned in (Lincoln, 2008). It can be concluded that Drupal is secure enough for production of live sites. The prototypes in this research are planned to be evaluated with designers from different practices. To reach out to them efficiently, the prototypes will be deployed and tested online. A secure website platform is therefore necessary to carry out this plan.

- Strong community support. The community has been an active and talking one, continuously improving the Drupal core as well as contributing additional features. The contributions are largely in the form of plugins/modules, which provide additional functionality to the core. The community runs an active forum that helps each other especially beginners to Drupal. Numerous commercial companies are also supporting Drupals by means of workshops/trainings, articles and consultancy about Drupal. With the apparent support from its community, the Drupal framework is considered reliable to be the platform for our prototype development.

Summarizing, the support tool is intended to work with a variety of design data, with a concern that these data might grow huge especially in complex projects. Drupal as a CMF has the quality to answer to these needs, and therefore is chosen as the platform to build prototypes of the support tool.

\subsubsection{The Development Steps}

The development using Drupal consists of two major steps. The first step focuses on getting a firm understanding of the core functionality of Drupal. To be able to extend the core functionality, the next step involves extensive research on additional Drupal modules that could be useful for the prototype development.

\section{Drupal Core}

The main requirement of the support tool matches well with the Drupal core functionality, i.e. to manage information-based content. The tool requires specific designation of data, which could be implemented by creating Drupal "content types". However, as a generic platform for Content Management Systems, the basic installation of Drupal core is plain and very limited for what we want to achieve with the prototype development. To illustrate this, Figure 6.2 shows the content template "story", one of the two basic content types provided in Drupal core. The core functionality of Drupal does not sufficiently accommodate our requirements, e.g. composing content types with more elaborate information types, creating explicit relationships between content types, etc. These functional requirements as well as other technical ones can be answered by additional modules which are contributed by the community. 


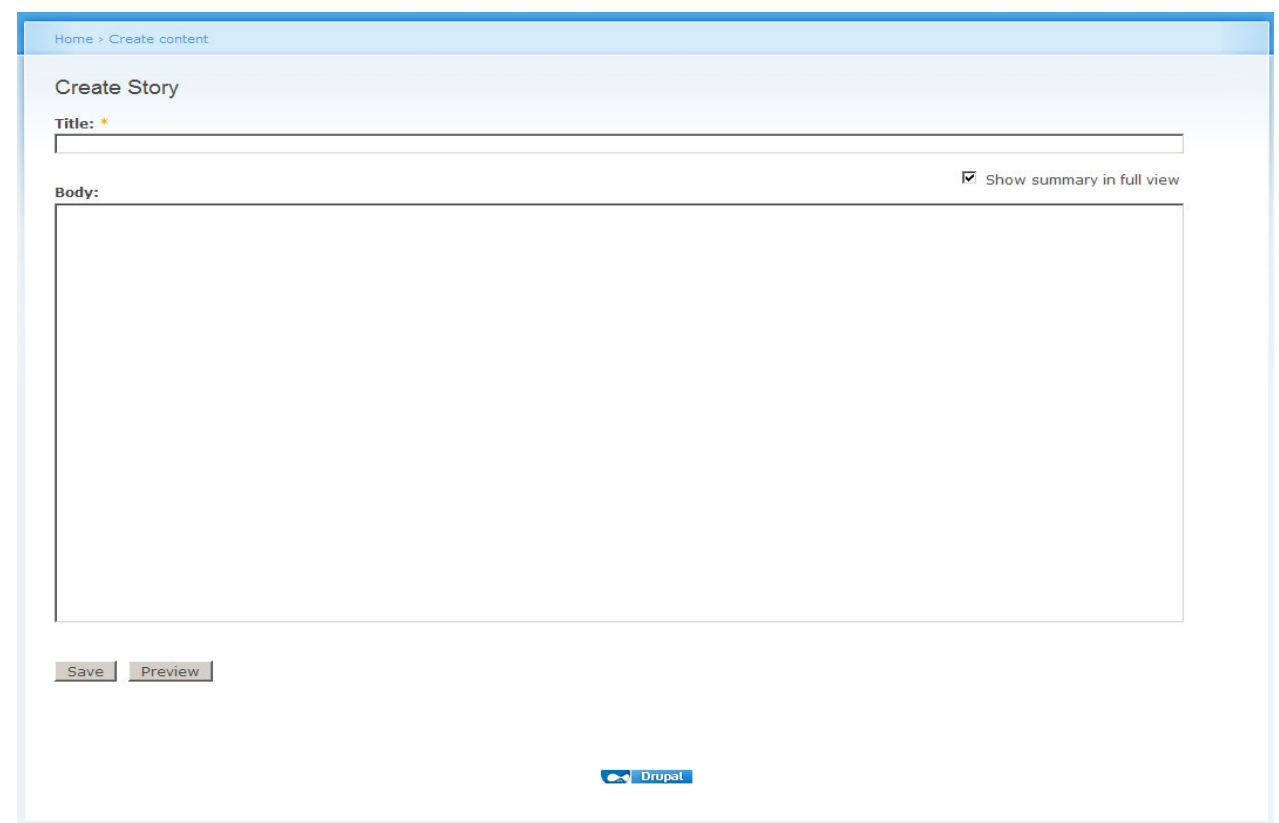

Figure 6.2: The creation of content type "story" in the basic installation of Drupal; the other content type, "page", is a more static version of "story".

\section{Additional Modules}

In the selection of the additional modules, we use the following criteria to assess the credibility of each module:

- The availability of a complete documentation that clarifies the module functionality. It should also explain the necessary steps to install, use and configure the module.

- Activities around the module (e.g. number of downloads, bug reports, open and solved issues, troubleshooting questions and answers).

- User reviews.

After a thorough research on Drupal core and modules, several modules have been selected since they may provide partial solutions in the implementation of the tool. These findings reaffirm Drupal as the platform for implementing the prototypes. Although it is possible to extend the modules or contribute new ones to the community, it is not feasible due to time and resource constraints. Nevertheless, we consider the available modules sufficient for the prototype development.

\subsection{Conclusion}

This chapter has delivered the evaluation setup for the support tool. To keep in touch with the reality of design practice, the evaluation will involve target users who represent the varieties in practice, i.e. varied levels of experience and different contexts (work practice/organization culture). An interactive prototype is needed to conduct this evaluation. Upon researching the available technologies, Drupal - an open-source Content Management Framework, is chosen to implement this interactive prototype. As a development platform, Drupal offers a balance between customizable functionality and ease of use in development. Furthermore, its community has been actively contributing additional modules that extend the core functionality of Drupal. Altogether Drupal and its supporting modules are capable of supporting the prototype development in this research. The next chapter will describe an interactive prototype built with Drupal and its evaluation with two different groups of designers: novice and experienced in using scenarios. 


\section{Intermediate Prototype and Evaluation}

Chapter 5 and Chapter 6 have described the conceptual design of the tool and the implementation plan respectively. As elaborated in Chapter 6, the evaluation criteria require a more interactive prototype; Drupal is chosen as a platform to implement this prototype. This intermediate evaluation aims to verify the implemented functionality so far with design practitioners as our target users. To cover realistic feedback from practice, this evaluation involves two different target groups in two separate sessions, one with less experienced general designers and another with designers more experienced in applying scenario-based approaches. The feedback from both evaluation sessions will be integrated in the next development steps.

This chapter describes our evaluation approach using the intermediate prototype, and concludes with the findings from the evaluation.

\subsection{Intermediate Prototype}

The prototype developed using Drupal gives a mostly accurate portrayal of the supposed interaction and use of the support tool. As a consistent implementation of the conceptual design, the intermediate prototype encompasses the most parts of the functionality of the support tool as shown in Figure 7.1. The prototype intentionally excludes the use case "deliver information". As explained in Chapter 5, this use case is not addressed in the intermediate prototype because it concerns a practical aspect of the tool and its implementation would be organization-specific.

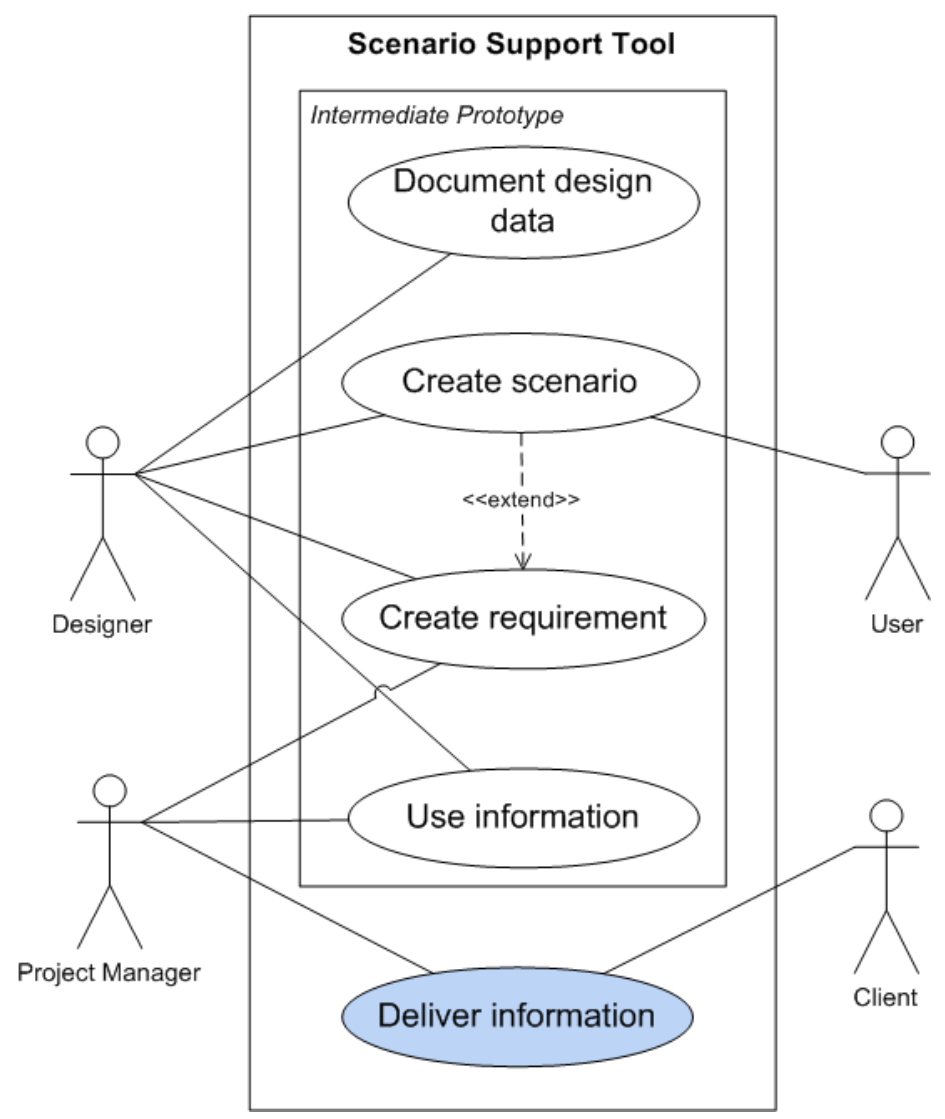

Figure 7.1: The overview of functionality as implemented in the intermediate prototype. 
To illustrate the information flow using the tool, a fictive case of designing an intelligent night lamp for users with limited abilities is used. The case provides a broader possibility of interactions, and is general enough to be understood by the participants in both evaluation sessions. Therefore it has been chosen over the case bike luggage transporter, which has appeared in the requirement identification (Chapter 4) and conceptual design (Chapter 5). This intermediate prototype shares a large similarity with the final prototype (Chapter 8), as it is a work-in-progress towards the final prototype. To avoid repetition, Figure 7.2 to Figure 7.7 show the basic functions to illustrate the operational state of the prototype. To get a more thorough overview of the complete implemented functionality, please see the description of the final prototype in Chapter 8. As far as functionality is concerned, both the intermediate and final prototypes implement the functionality as described in Chapter 5 consistently. This section only shows parts of the intermediate prototype to highlight that it is a more basic and less complete version of the final prototype. 
Janneke View Edit Clone

Nickname: *

Janneke

Profile picture:

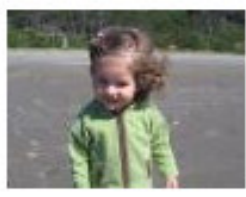

Remove

Keywords:

\begin{tabular}{|l|l|}
\hline <none> & Add \\
\hline age category > toddler & Remove \\
\hline attitude > shy & Remove \\
\hline gender $>$ female & Remove \\
\hline
\end{tabular}

Keywords that describe this actor.

D More keywords in categories

Information about this actor:

ॠ Show summary in full view

Jlanneke is a 4 year old girl, shy and timid, a bit clingy to her mom. She is an only child, attends playgroup and daycare 3 times a week. She likes to be read stories by her parents before bedtime. She has problems with sleeping, and often wakes up in the middle of the night. Her fear is being alone in the dark.

Enable rich-text

Save Preview Delete

Figure 7.2: An overview of creating an actor profile using the intermediate prototype; the link "More keywords in categories" can be expanded (Figure 7.3). 


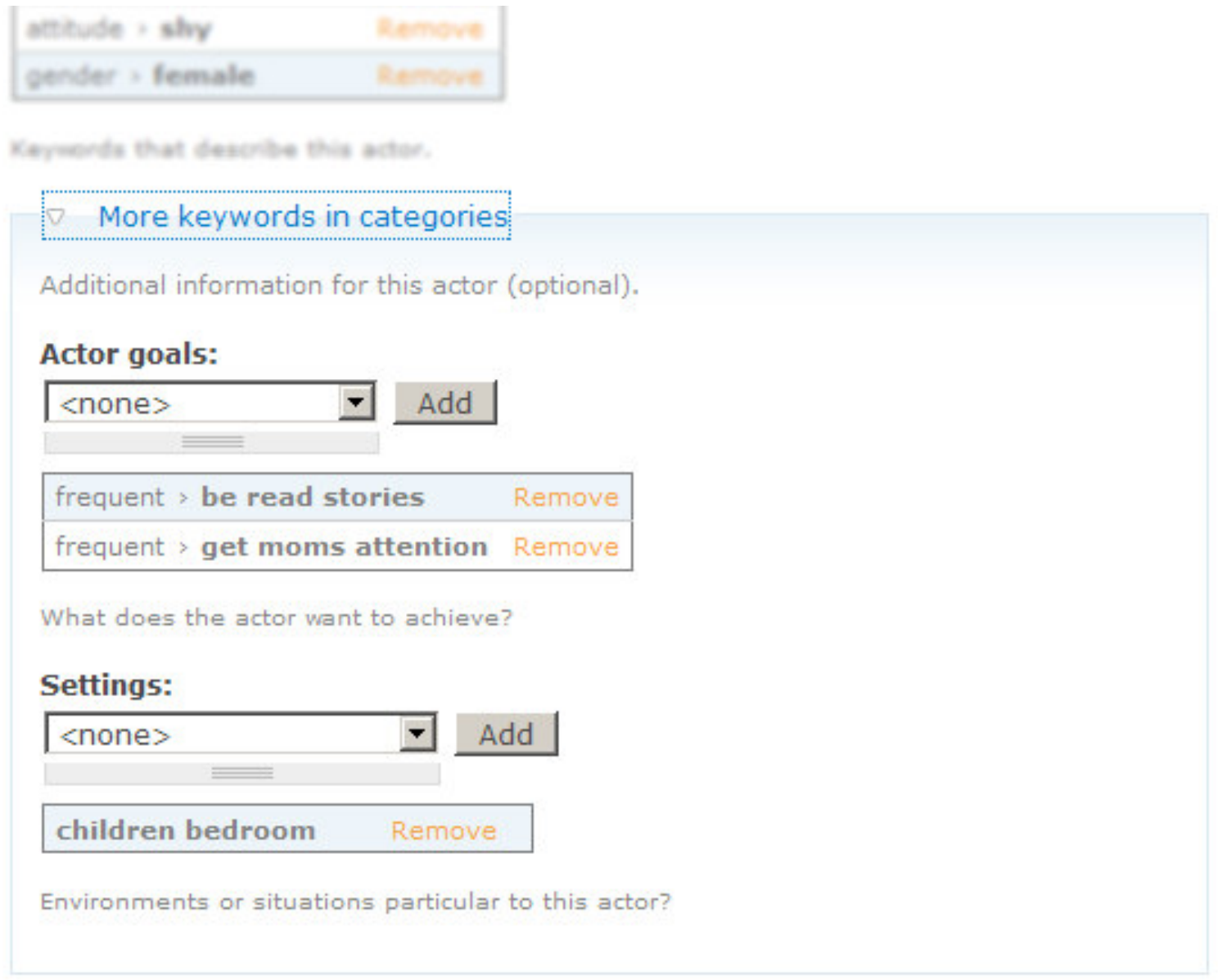

Information about this actor:

Show summary in full view

Danneke is a 4 vear old gil, shy and timid, a bit clingy to her mom. She is an lonly child. attends plaroroup and darcare 3 times a week. She thes to be

Figure 7.3: In an actor profile, a set of actor goals and typical settings can be specified. 


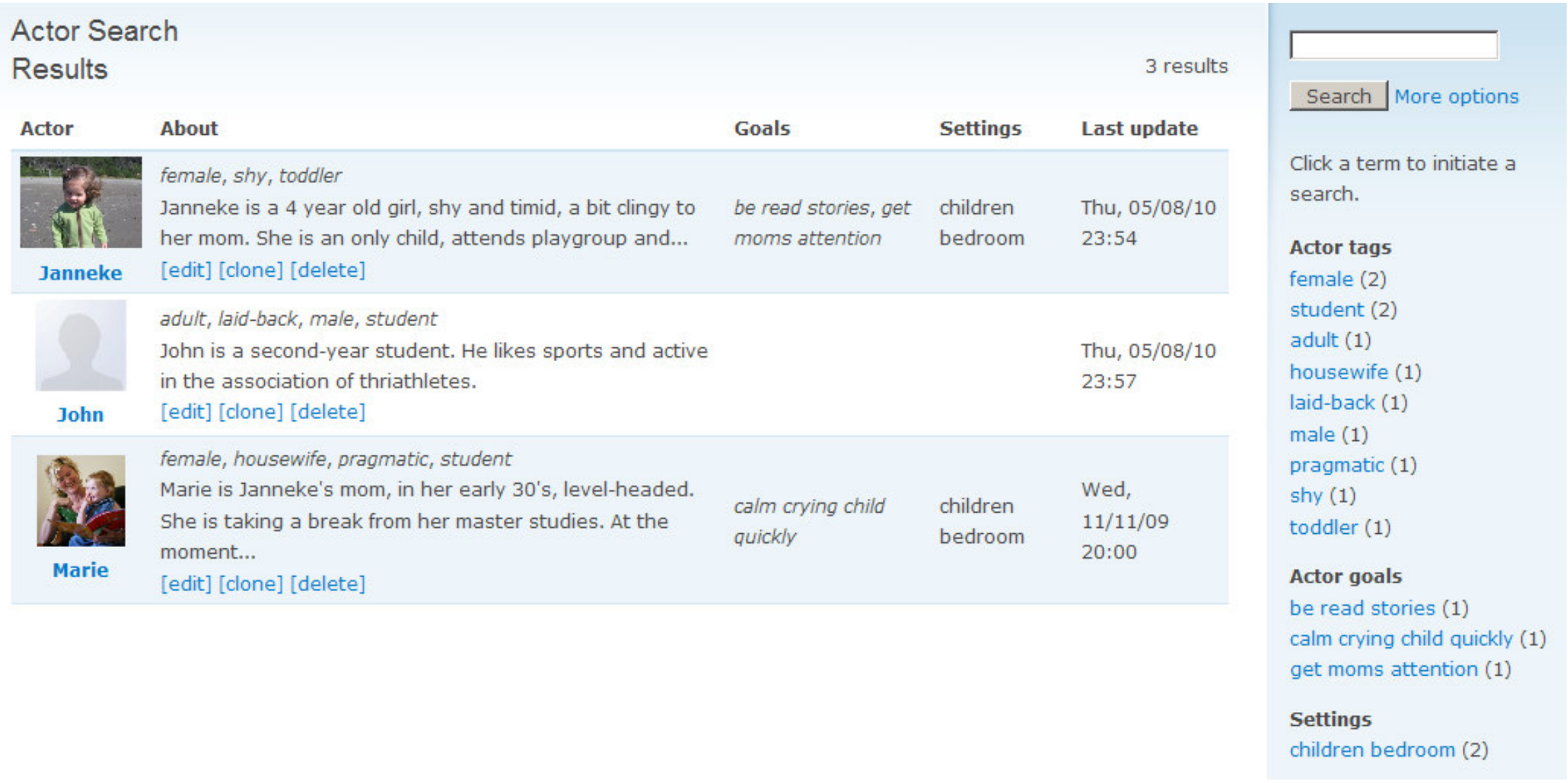

Figure 7.4: Displaying all actor profiles; clicking on any keyword on the right side will update the search result. 
hell breaks loose. View Edit Clone

The combinations of the recorded elements (i.e. actors, goals, products, settings, events) could inspire many possible scenarios. To start a scenario, it needs the necessary elements: an actor and his/her goal, the tool/product being used and a setting.

Other elements (e.g. events) could be included to create variants of a normal scenario.

Write the basic storyline of this scenario. Be as concrete as possible though you don't need to write a detailed story at first try. If you find yourself thinking of another story: finish this one first, and create another scenario for the variant you thought of just now.

A descriptive title to the scenario:

hell breaks loose.

$\nabla$ Some important details

Add extra information to distinguish this scenario

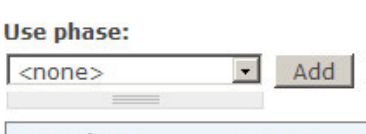

critical use Remove

Particular phase (s) in which this scenario occurs

Group:

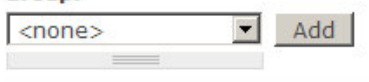

Nothing has been selected.

Assign group labels to this scenario

Components of this scenario

What's the story?:

V Show summary in full view

Disaster strikes; ]anneke spent the day at her grandparents playing in the yard. At 8 o clock it's time for bed. Unfortunately, her teddie bear Bernie is

probably left somewhere in the yard and nowhere to be found.

Janneke wakes up in the middle of the night and reaches out for bernie who is not there, and is aware of her being alone in a strange dark environment. She starts crying.

Enable rich-text

Save Preview Delete

Figure 7.6: A scenario reuses the documented information (e.g. actor, product, settings, etc) as its building elements. 


\begin{tabular}{|c|c|c|c|}
\hline \multicolumn{4}{|l|}{ Scenario Search } \\
\hline Scenario & Brief & About & $\begin{array}{l}\text { Updated } \\
\text { date }\end{array}$ \\
\hline $\begin{array}{l}\text { hell breaks loose. } \\
\text { critical use } \\
\text { [edit] [clone] [delete] }\end{array}$ & $\begin{array}{l}\text { Jin Janneke } \\
\text { intelligent night amp } \\
\text { get moms attention }\end{array}$ & $\begin{array}{l}\text { waking up in the middle of the night } \\
\text { at grandparent's, unfamiliar atmosphere } \\
\text { Disaster strikes; Janneke spent the day at her } \\
\text { grandparents playing in the yard. At } 8 \text { o clock it's time } \\
\text { for bed.... }\end{array}$ & $\begin{array}{l}\text { Fri, 06/08/10 } \\
0: 33\end{array}$ \\
\hline $\begin{array}{l}\text { Janneke stays in bed } \\
\text { mesmerized by the } \\
\text { pulsating light } \\
\text { daily use } \\
\text { [edit] [clone] [delete] }\end{array}$ & $\begin{array}{l}\text { IIt Janneke } \\
\text { stay in bed }\end{array}$ & $\begin{array}{l}\text { morning break } \\
\text { children bedroom } \\
\text { Janneke wakes up in the morning but the lamp having } \\
\text { been set to a certain time exudes a mesmerizing } \\
\text { miasma of light... }\end{array}$ & $\begin{array}{l}\text { Fri, } 06 / 08 / 10 \\
0: 35\end{array}$ \\
\hline
\end{tabular}

Search More options

Click a term to initiate a search.

Scenario use phases

daily use (1)

critical use (1)

Actor goals

get moms attention (1)

stay in bed (1)

\section{Events}

morning break (1)

waking up in the middle of the night (1)

\section{Settings}

at grandparent's (1)

children bedroom (1)

unfamiliar atmosphere (1)

Figure 7.7: Displaying all scenarios; clicking on any keyword on the right side will update the search result. 
The intermediate prototype includes only the basic features of the support tool. It is nevertheless interactive enough to allow the verification of the main functionality and interaction. The objective of the evaluation is to elicit qualitative feedback from the designers, which will be incorporated in the next development steps. Therefore, the main evaluation approach is centred upon show-and-tell of the functionality and discussions, while giving opportunity to the designers to try the prototype themselves whenever feasible. In doing so, the designers could reflect on their practice and give concrete feedback. The evaluation consists of two parts, a workshop (a part of a larger symposium) with general designers and a focus group meeting involving the designers at Indes. The next sections describe the setup and findings of both evaluation sessions.

\subsection{Evaluation 1: The Usability Workshop with General Designers (novice)}

With a symposium organized by TU Delft in celebration of the World's Usability Day (12 November 2009), there is an opportunity to hold a workshop with the theme 'usability'. Since the workshop addresses usability on a beginner level, a general audience from the design industry is expected. We use this opportunity to evaluate the prototype together with designers who are less experienced with usability in general and scenariobased design in particular. By conducting the workshop participants, this research fulfils an evaluation with one variety of target users, i.e. the less experienced ones, as mentioned in Chapter 6. The evaluation aims to check whether the support tool could be useful in guiding general designers to become more aware of usability through the use of scenarios.

The workshop introduces the basics of design for usability by means of a lecture and two assignments. The second assignment deals specifically with managing design information and creating scenarios using the tool developed in this research. The hypothetical use of the tool is demonstrated by means of the prototype using a fictive design case and design data. Afterwards, the participants perform small tasks using the prototype to document design data, compose and organize scenarios. The researcher engages the participants informally to elicit feedback while they are trying out the prototype. Appendix 3 provides the complete structure and tasks of the workshop.

The workshop brings together participants from various domains, mainly from the software industry. The majority is from web and software design domain, ranging from graphic/web designers to usability specialists. Only two participants are product designers, the actual target users of the tool. Nevertheless, all participants could accept the approach of identifying, creating and organizing scenarios for a design project, shown by their agreeing with the information flow presented in the tool prototype. From informal discussion with them, it can be concluded that the functionality answers to a realistic need in the design practice. The next section elaborates the topics that arise during the discussion with the workshop participants.

\subsection{Evaluation 1: Discussions}

The first topic raised by the participants concerns the target users of the tool. This confirms the need to evaluate the support tool with varied target users, as proposed in Chapter 6. The discussion gives an indication about the different perspectives occurring in different organization contexts. For instance, some of the participants work in large companies with a stronger hierarchy. These participants express that only team members with functions as usability expert or project manager could use the tool; the designers and developers could be left with the creative tasks. Other participants work at smaller design agencies or independently. In the practice of small to medium sized companies, the targeted setting of the tool, design teams tend to be small and consist of all-round designers. These all-round designers need to know the overview of the 
design process, not only the particular activities of designing. In other activities such as in formulating requirements or usability testing, they would appreciate support that the tool offers. This insight indicates that the tool can be useful in both settings, as long as the design approach focuses on users and usability.

Secondly, the participants expect that the structured approach of the tool to give insight into large complex projects. In such projects, the support tool can remind the designers where to look for information while building scenarios. For this purpose, the participants make some statements which are summarized as the following:

- The tool is useful for providing a checklist of the most relevant information needed for writing scenarios,

- The tool offers high flexibility in documenting data (by providing non-restrictive template),

- The tool encourages reusing design information, for instance personas (user descriptions) could be used throughout a whole project or across projects,

- To be useful in the (participant's) organization, the tool needs to incorporate information about the project (e.g. background, goals, and design principles/approach). This suggestion, although outside the scope of this research, reflects the practical values of the tool.

Lastly, owing to the high-level of fidelity of the prototype the participants give feedback to improve the interface and interaction of the prototype. In general, the feedback revolves around creating a better, more visual overview of the documented information, and creating printed output. These suggestions confirm the findings from the evaluation of the conceptual prototype (Chapter 5). As explained in Chapter 5, the visualization and creation of outputs are practice-oriented and therefore outside the scope of this research. Additionally, an idea for an alternative layout has been explored with some participants. The idea is to provide a blank workspace for composing scenarios (as alternative to the current form-filling template) for the designers with more experience in using scenarios. This alternative layout is illustrated in Figure 7.8.

Summarizing, the overall feedback indicates that the support tool offers added values in the design practice at large. The participants have shown acceptance towards the basic functionality as implemented in the prototype. Additionally, they were also motivated to propose additional functions based on the currently implemented functionality. This verifies that the development is so far in the right direction. For the next planned evaluation with Indes, no major modification in the prototype is performed. Only a conceptual idea of an alternative layout is added to explore new possibilities for interface and interaction, as shown in Figure 7.8. The next subsection describes the evaluation with Indes by means of a focus group meeting.

\subsection{Evaluation 2: Focus Group Meeting with Indes Designers (experienced)}

The focus group meeting with Indes aims at four closely related purposes. First and foremost, the main purpose is to verify the correctness of the implemented functionality. The translation from identified requirements, proposed functionality, and finally towards the implementation needs to be checked for coherence. Secondly, by keeping the designers informed on the development progress, the meeting also expects to probe for more information about where additional functionality might be needed. Thirdly, even with the functionality confirmed, the interface and interaction also needs to be evaluated for its accessibility, effectiveness and efficiency. Feedback on this will be used to improve the next prototype. Lastly, an additional purpose is to get an indication 
on the overall quality of the prototype by means of questionnaire. The same questionnaire will be used in all stages of evaluation. Therefore, the questionnaire results will be a quantified measure that can be compared with the next version of the prototype.

Using the intermediate prototype, a focus group meeting is conducted with designers at Indes. The meeting is expected to generate more input on additional functionality, and feedback on the interface and interaction. The meeting is structured into three parts: (1) a presentation and demonstration of the tool functionality with examples using fictional data, followed by (2) open discussions, and concluded with (3) questionnaires.

Earlier in the conceptual phase, the tool functionality has been presented to and discussed with the designers at the company (Chapter 5). Therefore in this evaluation, with a demonstration of the web-based prototype, the designers confirm whether the functionality is implemented the way the designers expect it. The discussion that follows afterwards addresses more detailed interactions and interfaces that fit better with the designers' preferred way of working. To facilitate this, lectures handouts that contain screenshots of the current prototype are distributed at the start of the session. The participants are instructed that they could freely annotate and sketched on the handouts. The alternative layout (Figure 7.8), that has been discussed at the usability workshop, is also included in the handouts and discussed with the designers. The intention is to get additional feedback to improve the interface and interaction of the prototype. After the discussion is concluded, the designers fill in the questionnaire, giving their interim scores on the qualities of the prototype.

The focus group meeting brings together 9 participants. They have varied functions at the company, among others as designer, usability engineer, electronic engineer, and project manager. The design methodology of Indes already incorporates scenario uses; therefore all participants have background knowledge about scenario-based design approaches. However, due to their variable levels of experience and involvement in projects, they have been exposed to scenarios and usability-related design issues at different levels. The presentation and demonstration successfully bring the participants' understanding on a common level despite their different functions. By the time for discussion, everyone no matter of the function realizes the potentials of using the tool to support the process within the design team. For instance, an electronic engineer who is not directly involved with the users could foresee how being informed about users and use situations (in the form of scenarios) helps him justify his decisions. The discussion engages the perspectives of different functions, and delivers the expected feedback. The important findings from the discussion and the questionnaire results are presented in the next section. 


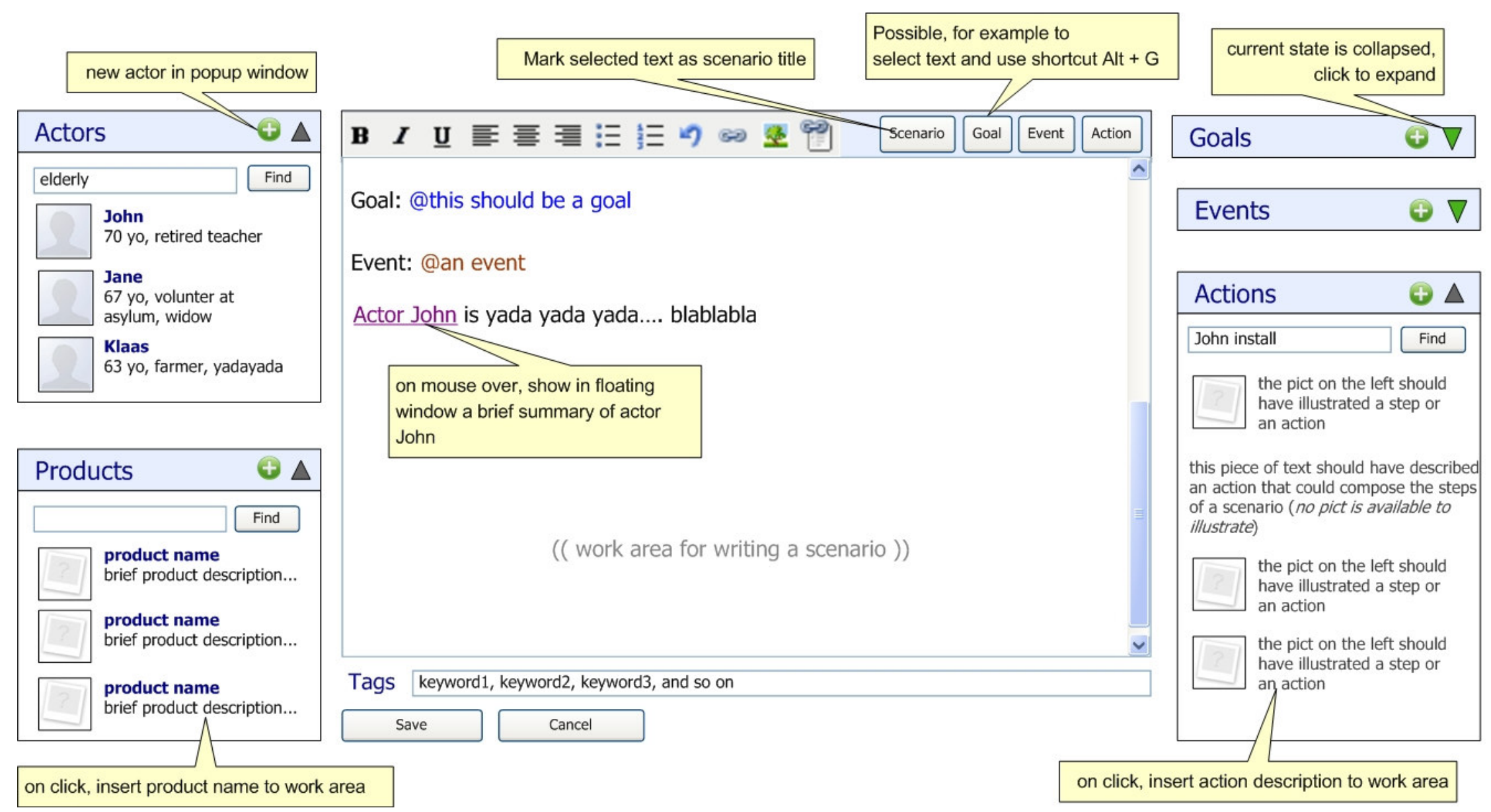

Figure 7.8: Alternative layout of the tool, based on the input from the Usability Workshop, shows a working area to compose scenarios and side boxes containing scenario elements which can be picked out. 


\subsection{Evaluation 2: Findings}

The findings will be divided into two parts. The first and most important result in this evaluation phase is the designers' feedback on the tool functionality and interaction. This feedback will be used to further improve the prototype. Secondly, the questionnaire results are discussed to get an indication of the overall quality of the tool.

\subsubsection{Feedback for Development}

The evaluation has delivered a number of qualitative feedbacks from the designers. The currently implemented functionality has been accepted and the added values recognized. The current practice at Indes uses an array of MS Office programs to document projects. Compared to this current way of documenting, the tool will offer several benefits:

- Reduced time for documentation: every team member can contribute easily without being constrained with standard documentation procedure,

- Reduced time for data (re)collection: the data is neatly stored in categories,

- Flexibility in documenting, collecting and representing data: various entry points, no prescriptive sequence; the alternative layout (Figure 7.8) will support such flexibility,

- Inspiration and verification: the availability of smart filtering and searching function,

- Increased overview: all data is in one system, and the relation between them can be made explicit.

More benefits are expected when the complete functionality (as described in Chapter 5 ) is fully implemented. The functions related to these benefits however, are mainly practice-oriented, i.e. their implementation will depend on the practice of the particular organization. They are not within the scope of this research, but mentioned here to reaffirm the feedback from the earlier evaluation (Chapter 5). In brief, further development of the tool for practical implementation would deliver these following benefits:

- Reuse of information (about users/actors and contexts) over different projects,

- Connection to other formal documents: the processed data could provide rationales for e.g. requirements and risk management,

- Visualizing information: information about users/actors, relations between actors and other information are more graspable.

- Delivery of output in various formats: the output will be useful for digital presentations (MS PowerPoint, JPG), formal reports (MS Word, PDF), or printouts (e.g. actor cards for brainstorming)

- Network of information: a possibility to link with information in other media (e.g. video, images, documents, online resources)

- Manageable access to information: a possibility to implement different views depending on e.g. roles (managers, tester, engineer), level of experience (newbie, advanced), and involvement in specific projects.

Overall, the feedback from Indes designers has answered three of the four aims for conducting the focus group meeting. Firstly, the correctness of the implemented functionality has been confirmed. This confirmation gives a foundation to continue with the planned steps in the development. Secondly, additional functionality has been explored. Most of the proposed functions have been discovered during the conceptual evaluation (Chapter 5). Since they are practice-oriented, they are not included in the 
next development steps. Thirdly, the interface/interaction of the prototype does not raise any question during the demonstration. It can be concluded that the interface/interaction has been direct and easy to understand. Furthermore, the alternative layout as proposed in Figure 7.8 has been discussed. The designers find the work area using 'blank canvas' metaphor will be useful as they become more familiar with the tool. It allows simpler, more flexible, and more intuitive interaction while composing scenarios. This layout will be implemented in the next development steps.

In addition to this feedback, the designers are also asked to score the prototype by means of a questionnaire. The questionnaire results are discussed in the next subsection to give an idea on the perceived overall quality of the prototype.

\subsubsection{Questionnaires}

The goal the questionnaire is to get an indication on the overall quality of the prototype. Since the same questionnaire will be used in all stages of evaluation, the results will be a quantified measure that can be compared with the next version of the prototype. The questionnaire assesses five qualities/categories, which are adapted from the evaluation criteria in Chapter 6:

1. The functionality category aims to find out the coverage of the general functionality,

2. The 'ease of learn and use' category evaluates how graspable and intuitive the use of the tool is,

3. The usefulness category aims to qualitatively measure the added values of the tool in practice both on the organizational or personal level,

4. The satisfaction category aims to measure more abstract qualities such as acceptance and preferences, and

5. The 'additional features' category aims to reconfirm the direction of future development, i.e. the functionality that is still planned for further implementation.

Each quality/category consists of three to seven questions, which will be addressed in each subsection. These questions are developed with the final tool in mind, and used consistently in all stages of evaluation. Therefore, depending on the particular stage of evaluation, some questions may not be relevant. The possible answer for each question is a score ranging from 1 ('strongly disagree') to 7 ('strongly agree'), plus an explanation when applies. For the analysis, the recorded score is the actual score minus 1 , so that 'strongly disagree' translates into 0 and 'strongly agree' into 6

As mentioned earlier, the nine participants have varied job functions, different levels of exposure to scenarios, and therefore variable opinions concerning the use of the tool in their specific tasks. The questionnaire scores are first scanned whether there is any sign of bias due to the participants' varied functions. A quick observation of the scores per user (Figure 7.9) shows little to no tendency of any biases, i.e. none of the participants consistently gave extremely high or low scores. All participants are able to asses the tool in terms of how it could support particular design projects or design teams, independent of their function. 


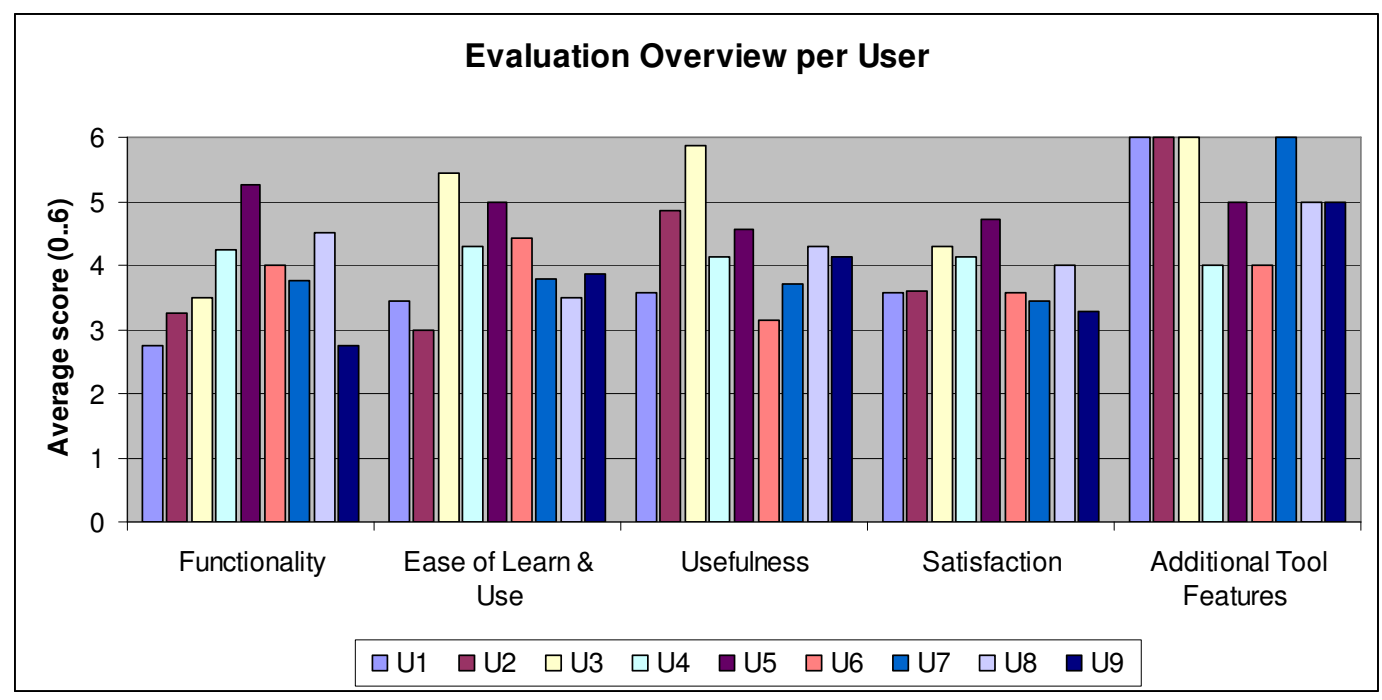

Figure 7.9: The overall quality scores per tester (score range 0 to 6, 'U1' stands for 'User 1').

The analysis focuses on the relative average score difference between the users (Figure 7.9). To start with, UI and U9 are experienced designers who have been using scenarios in various projects. They both find that the tool functionality is not fully sufficient to accommodate their wide range of needs, especially during the creative brainstorming or designing (related with the aforementioned lack of visualization). On the other hand, U8, a usability engineer, sees the functionality as more than sufficient in his line of works, a.o. identifying actors, defining use scenarios, and most importantly documenting them in the official project document. For the usability engineer, the tool is just another platform for his usual tasks, with more possibilities. Another category, usefulness, sees overall above average scores. Compared to the others, U2, an electronic specialist, sees the tool as very useful to give him an overview of the design project, although he may not participate in documenting information using the tool. A further analysis per category is available in Appendix 5.

The next development steps will implement the feedback as discussed during the focus group meeting. Therefore, the scores are expected to improve in the next evaluation phase. The most valuable information is that the additional features that are proposed on top of the implemented functionality have been well received (5.22 of maximum 6). This confirms the direction for the further development of the support tool.

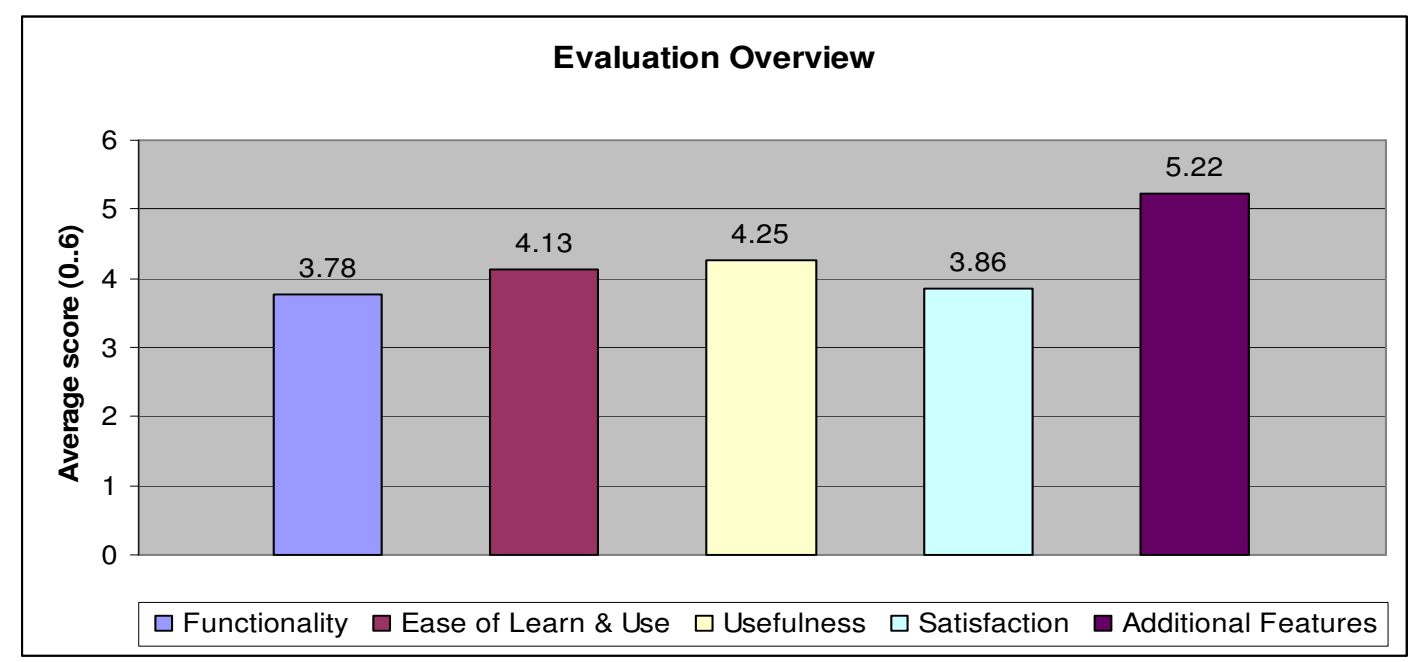

Figure 7.10: The overall scores for the evaluated qualities (score range 0 to 6 ). 
The questionnaire results confirm or complement those from the discussion, which are mainly in the form of qualitative feedback. One complementary finding, the category to assess the ease of learn/use of the tool has scored above average, indicating that the interaction/interface of the prototype is explanatory and easy to understand. While most categories are scored above average, there are certainly areas for improvement. For instance, the satisfaction-related question concerning whether the tool helps designers to be more creative (see Appendix 5) reveals a low average score. The intermediate prototype is perceived as too restrictive to allow creativity. From the discussion, this perception from the designers is related with the lack of visualization in the tool. Although visualization is not the focus of the development, this research is informed about the directions to improve the scores.

Overall, the questionnaire results have indicated the aspects of the tool that need more attention. Not all of them can be addressed in the next development steps. Nevertheless, they are acknowledged and will be included in recommendations when necessary. The next development steps will remain focused on implementing the main functionality, which has been reaffirmed in this intermediate evaluation.

\subsection{Conclusion}

This chapter has described the intermediate prototype and its evaluation with two different types of target users. Firstly, the usability workshop has probed the applicability of the support tool in the design practice at large, especially with designers from various practices who are generally less experienced in using scenarios. Secondly, the focus group meeting at Indes has involved designers who are more experienced with applying scenarios. The focus group meeting has confirmed the correctness of the implemented functionality so far in the intermediate prototype. To conclude, the workshop and the focus group meeting have delivered complementary findings that confirm the added values the support tool will bring in the design practice.

Furthermore, ideas for a better interaction with the tool have been explored, which includes an alternative layout of the main workspace. To reflect this on the choice of technology, the Drupal framework allows building the prototype quickly and not from complete scratch. On the downside, the Drupal modules used for building the prototype also determine its interface and interaction. While these modules are opensource and their codes may be modified by anyone; there will be limitations on what can be changed. The implementation of the additional ideas will be carried out as far as allowed by Drupal.

The next development steps will continue on implementing the main functionality. However, we will also opportunistically see where the implementation of these ideas can be applied. The next chapter will describe the resulting prototype in details. 

The intermediate evaluation as described in Chapter 7 has concluded with a set of findings and qualitative feedback to improve the support tool. To verify whether the feedback has been properly implemented, another round of evaluation has been conducted. An improved version of the prototype is developed for the evaluation. The final prototype encompasses most parts of the functionality of the support tool, as shown in Figure 8.1. Its implementation is consistent with the conceptual design and the earlier prototype. Similar to the intermediate prototype, this final prototype gives a mostly accurate portrayal of the supposed interaction and use of the support tool.

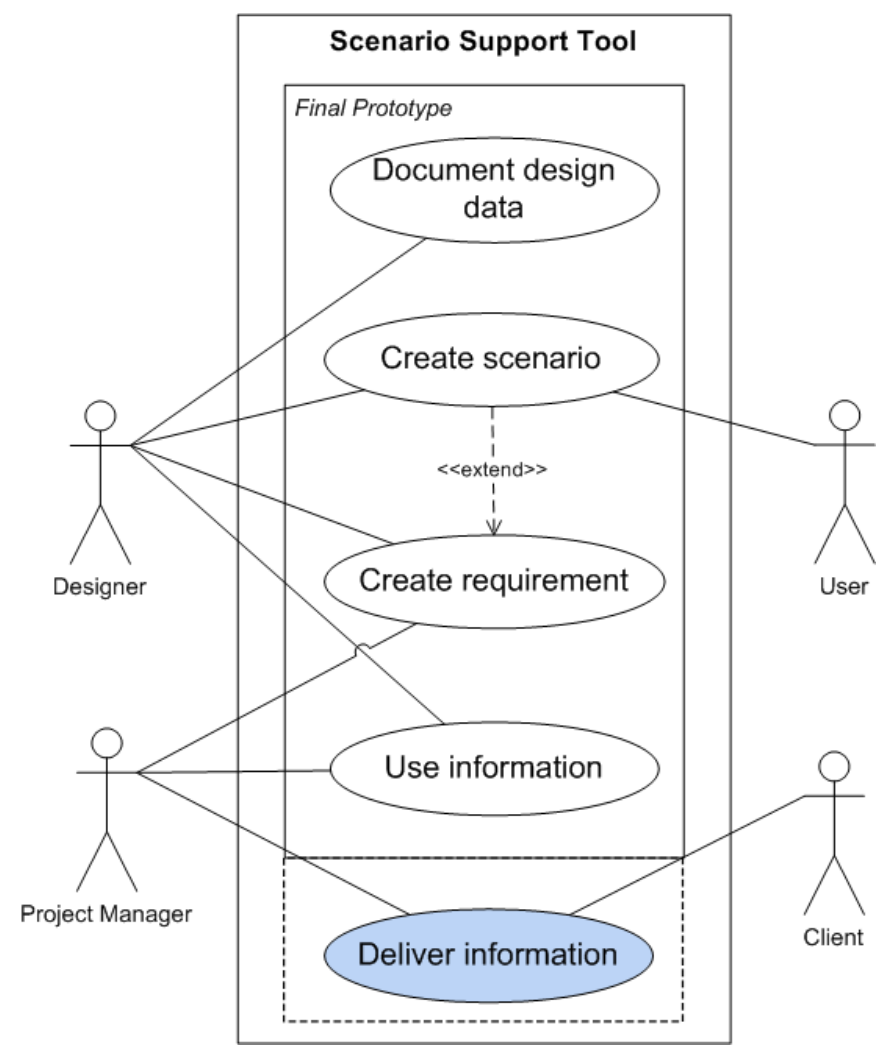

Figure 8.1: The overview of functionality as implemented in the final prototype.

However, unlike the intermediate prototype, the final prototype partially implements the use case "deliver information" as shown in the dotted box in Figure 8.1. This use case concerns a rather straightforward functionality, and its implementation would need to be based on what is requested by various design practices. Since the final prototype is developed to illustrate our approach and not to cater the specific requests of any design practice, the implementation is limited only to demonstrate the availability of the function to deliver information. In the final evaluation, the participants need to get a good overview of the support tool as a whole. Therefore this functionality is explicitly expressed in the final prototype, despite not fully implemented. Additionally, the final prototype also implements some details to improve the aspect of ease of learn/use. For instance, introductory pages are available in the prototype to guide firsttime users (designers) in understanding what the tool has to offer. Figure 8.2 and Figure 8.3 depict the first two pages that the use faces when using the tool, before and after login respectively. 


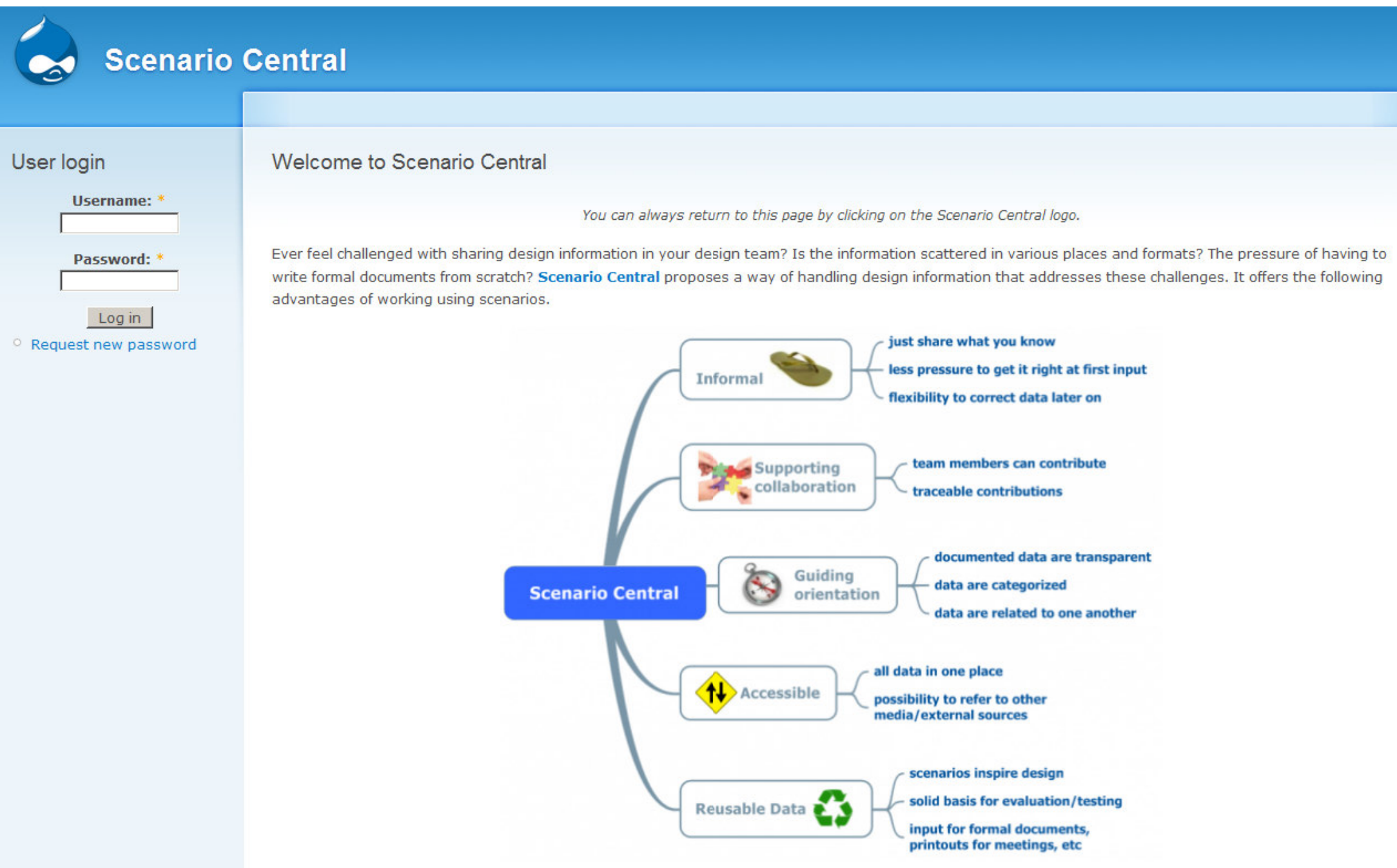

Scenario building is a natural part of designing for people. Scenario Central provides a structured yet non-rigid approach to use scenarios in a design project. An overview of scenario-based design is presented in the next page.

Figure 8.2: The welcome screen provides simple guidance on why and how to use the support tool. 


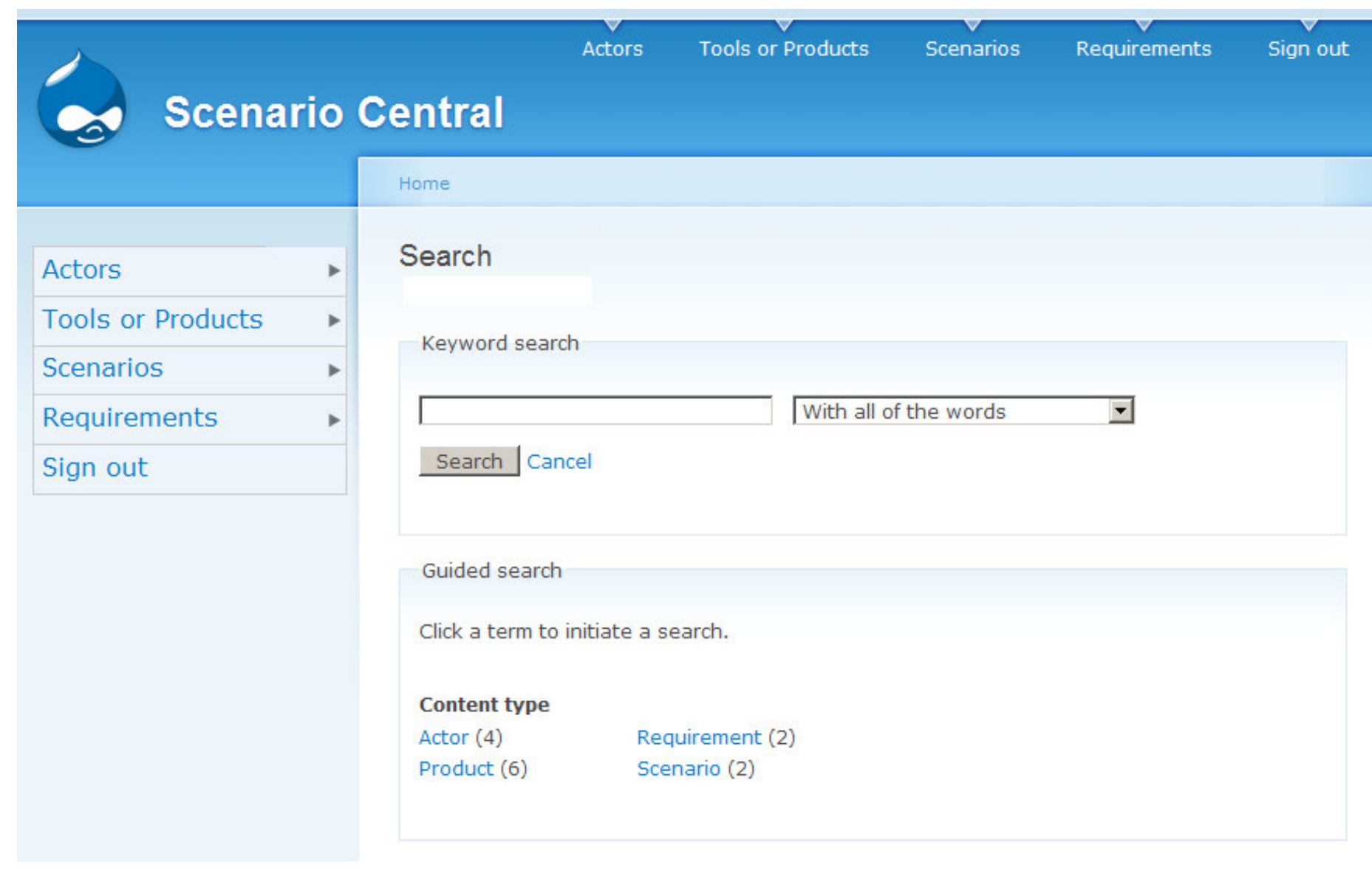

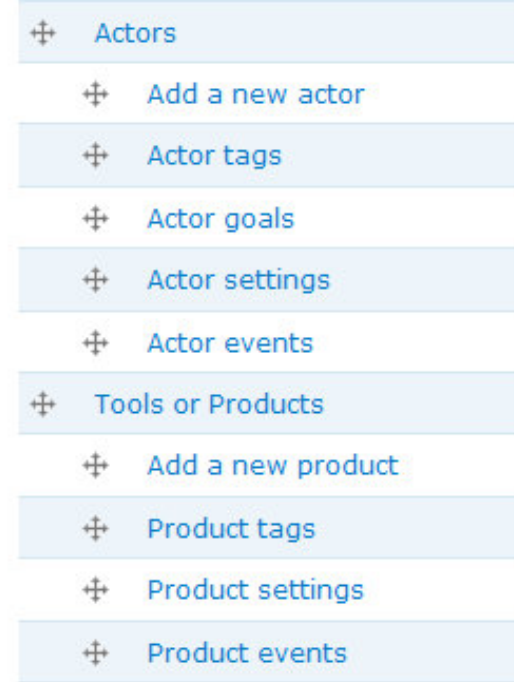

4 Scenarios

4 Write a scenario

屯 Scenario use phases

廿 Scenario groups

* Requirements

* Suggest a requirement

\$ Requirement categories

+ Sign out

Figure 8.3: After login the designer can see the overview of information already in the system and menus on the left and the top to access each specific type of information; the menu tree shows the complete navigation structure of the prototype (right). 
Apart from these introductory pages, the final prototype addresses the main functionality of the support tool. To maintain structure, the final prototype will be described per use case in the following sections. A detailed diagram in each section shows the extent of implemented functionality in the prototype. The same fictive case in Chapter 7, designing an intelligent night lamp for users with limited abilities, is used to illustrate the information flow using the tool.

\subsection{Use case: "Document design data"}

As elaborated in Chapter 5, the tool guides designers in documenting the necessary design information using scenario elements as inspiration. In practice most likely there already exist documented design data in different formats (e.g. documents, spreadsheet, etc). Therefore there is a practical need for a function to import existing design data into the support tool. This function is acknowledged and represented by the detailed use case "import design data". However, this function is not addressed in the final prototype (shown as the shadowy ellipse as shown in Figure 8.4). The reason is that the types, formats and meanings of the existing design data are variable depending on each specific practice. Implementing the function would mean customizing the prototype to different requests from each practitioner. Besides not being feasible, it is also not relevant with the purpose of this research. Figure 8.4 gives an overview of the functionality that has been implemented in the final prototype with respect to the use case 'document design data'.

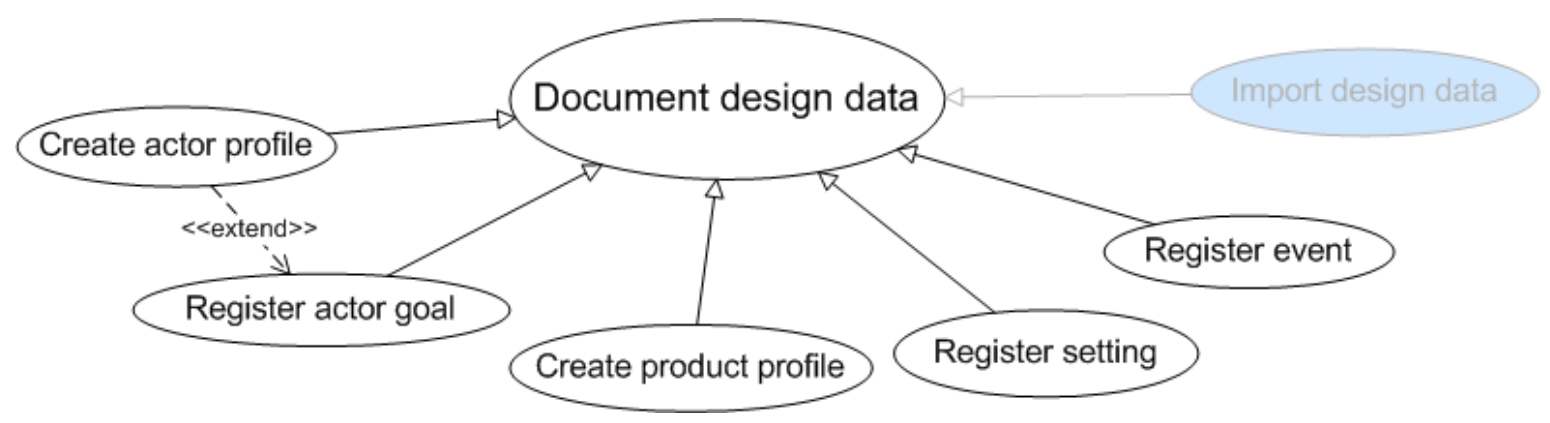

Figure 8.4: The implemented functions for use case "document design data" in the final prototype; the greyed out use case "import design data" is intentionally excluded.

Figure 8.6 to Figure 8.16 displays the parts of the prototype that address the documentation of design data. Some of the figures do not directly represent the functions covered in the use case "document design data", but are included to provide a coherent story about the use of the support tool. An example of such figures is the following Figure 8.5. 


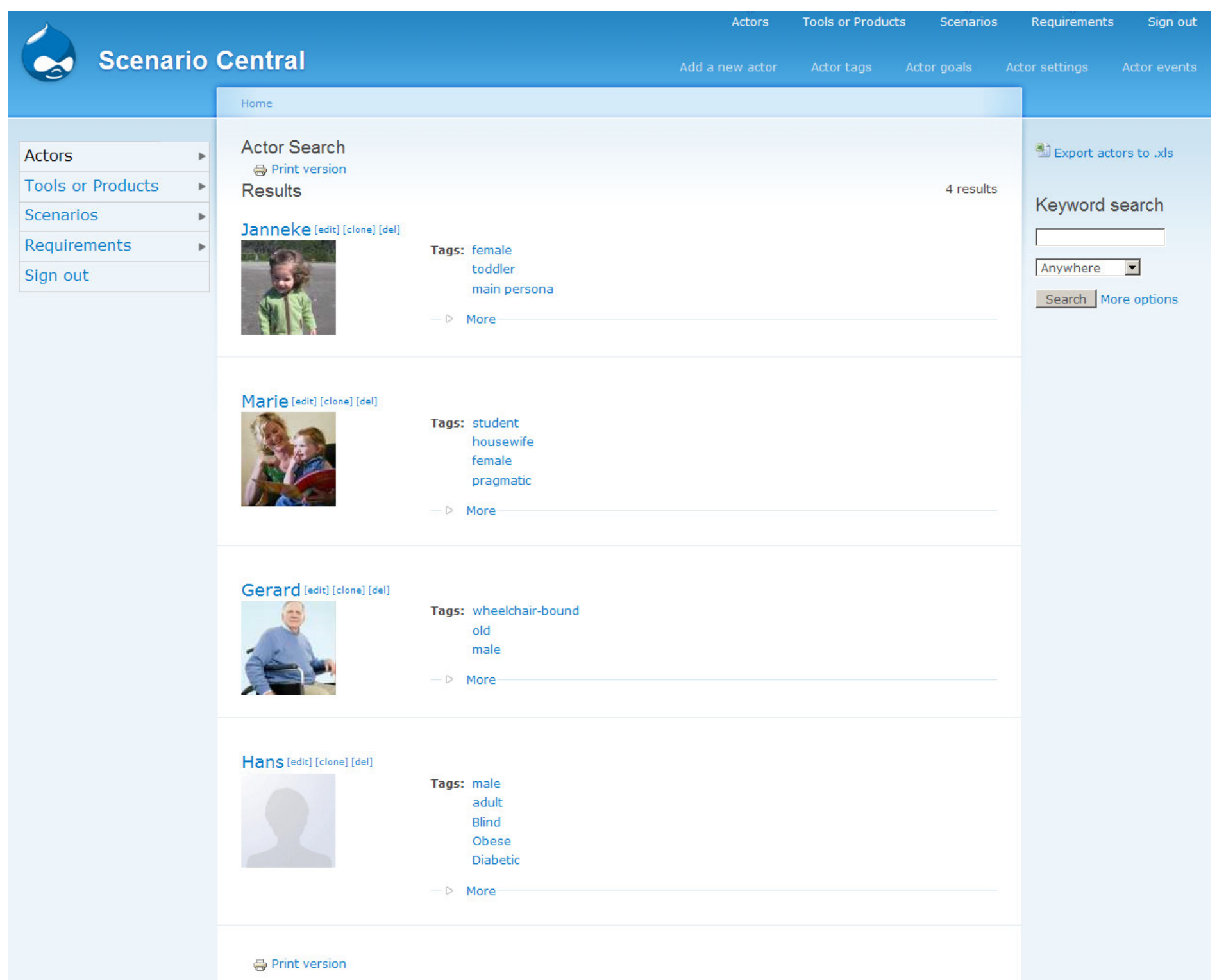

Figure 8.5: Displaying the list of actors, each of which has links for edit, duplicate (clone) and delete; adding a new actor profile via the menu link on the left sidebar or top header. 


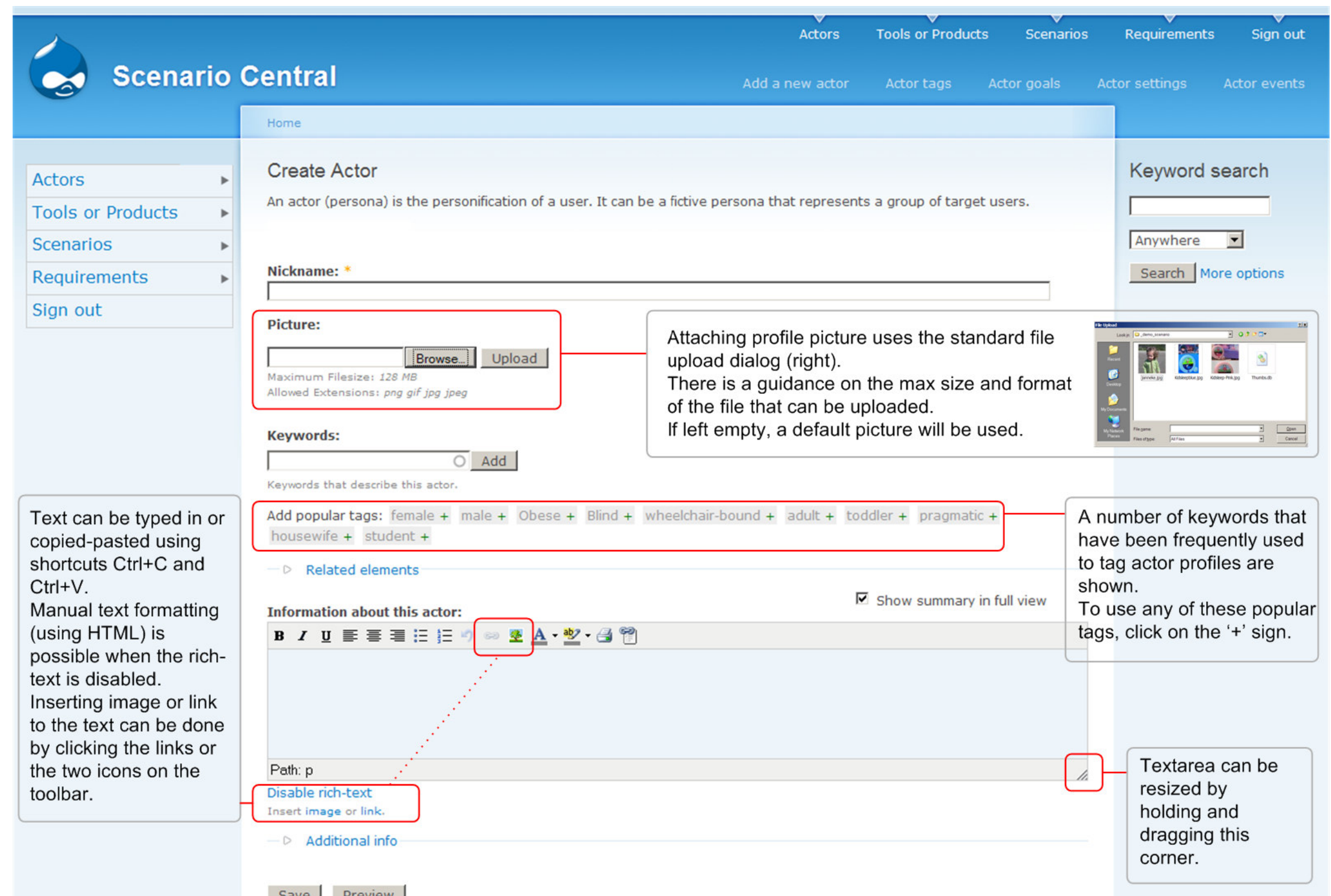

Figure 8.6: An overview of creating a new actor profile with some explanations on the interfaces. 


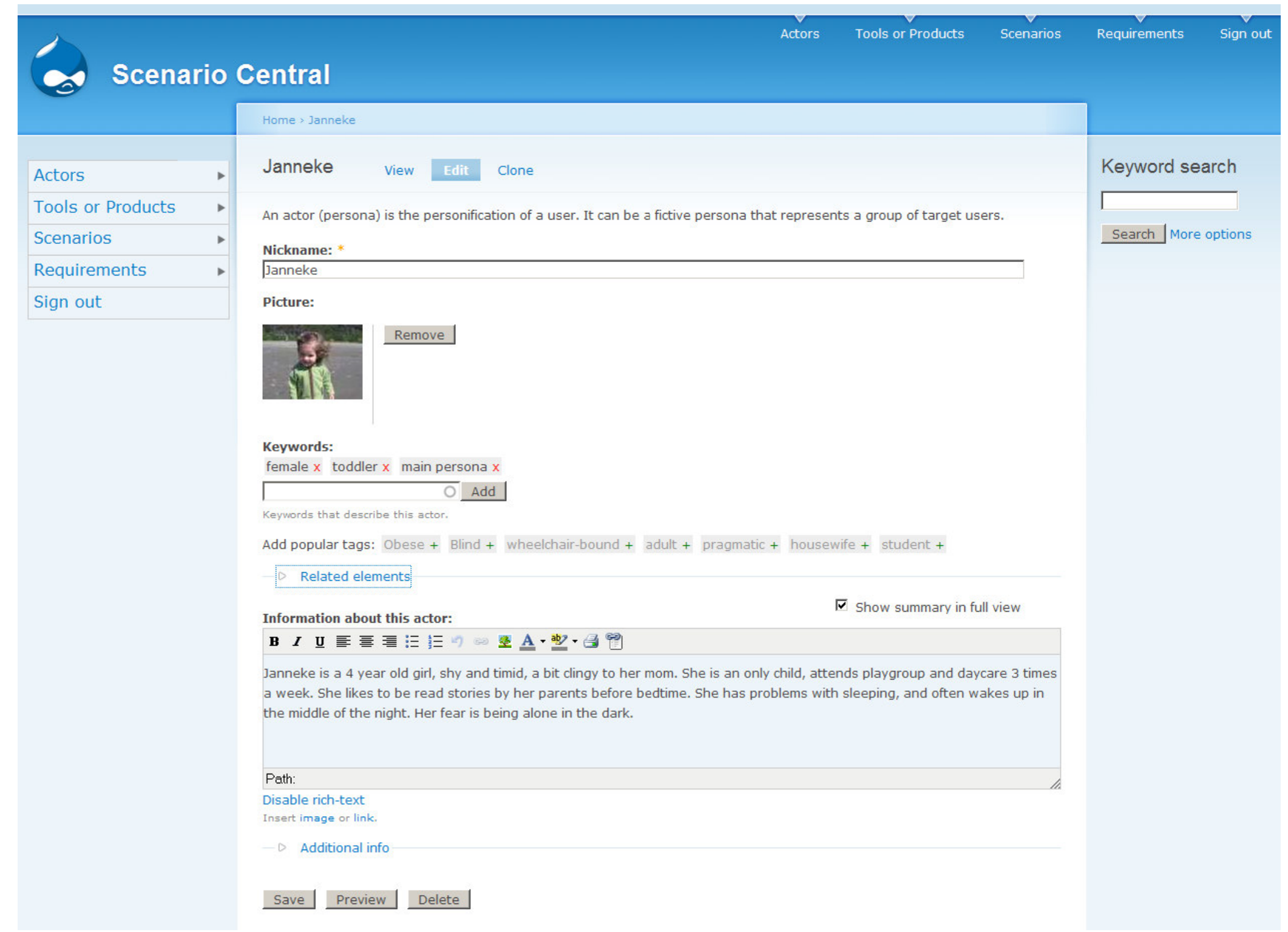




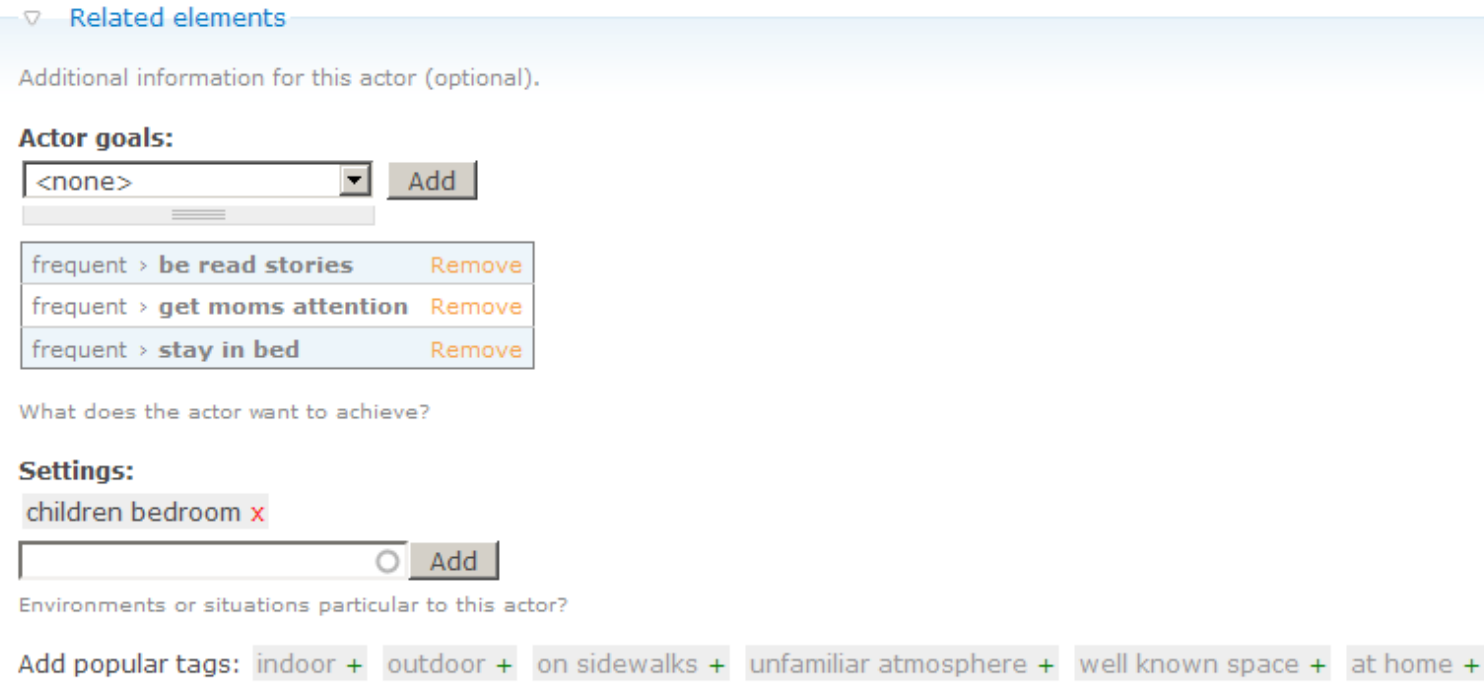

Figure 8.8: In an actor profile, other scenario elements such as actor goals and settings can be specified.

\section{Additional info}

\section{Add additional docs to describe the product (optional).}

Attachments:

Order

屯 Browse... Upload

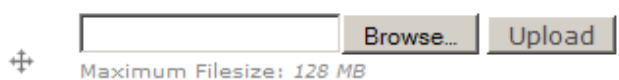

Figure 8.9: External documents can be attached to an actor profile; thus not constraining the use of other programs/tools. 


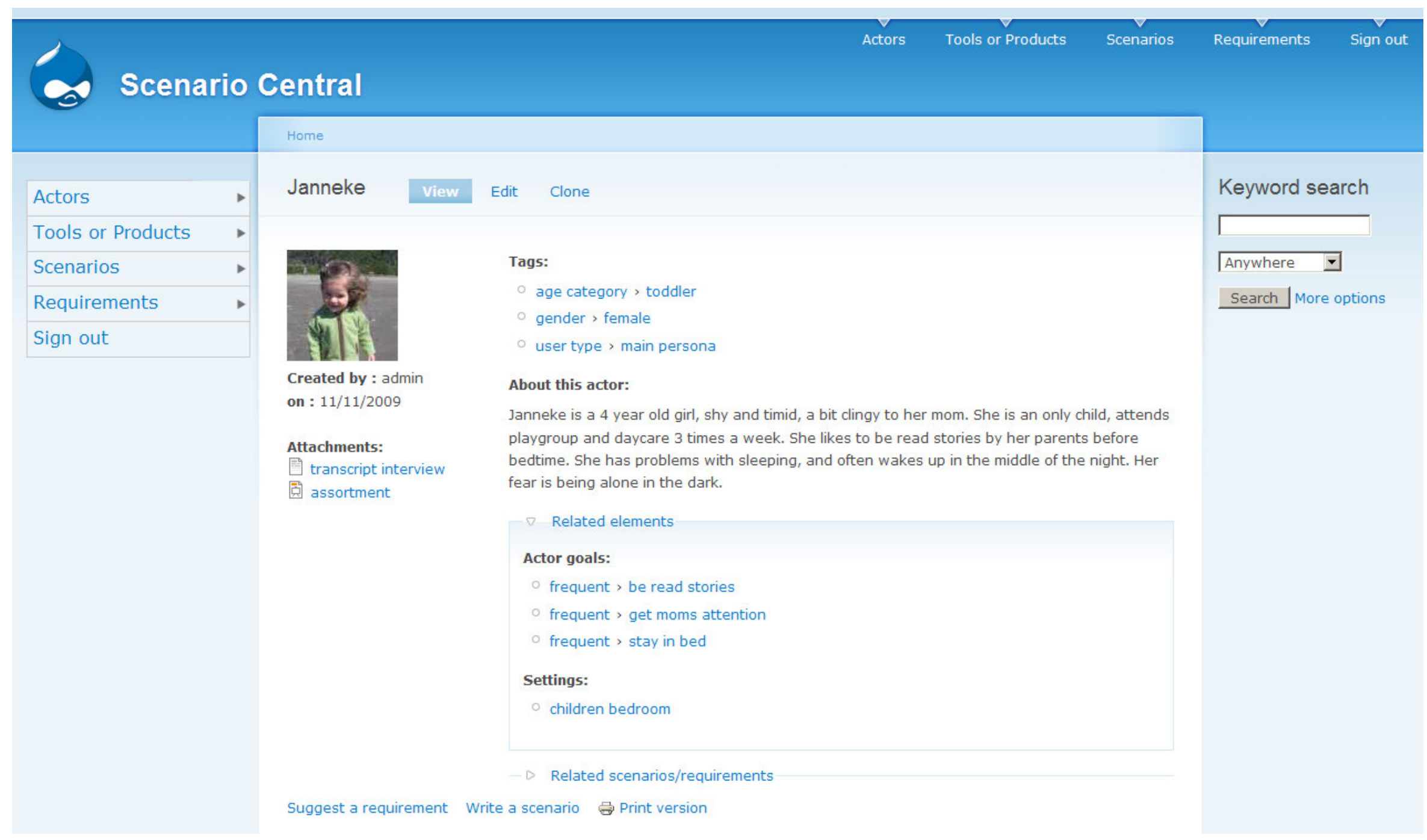




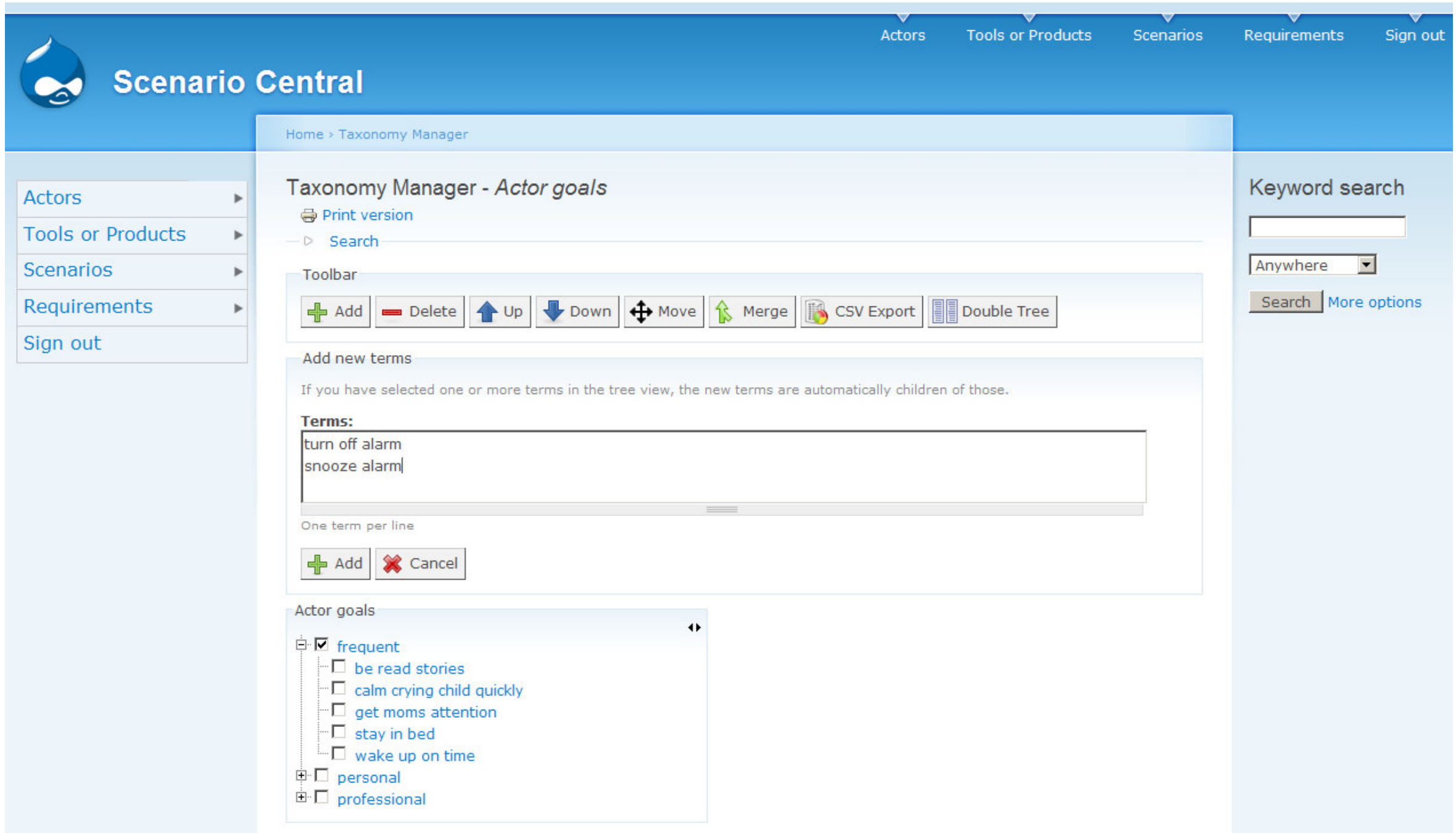

Figure 8.11: Actor goals can be managed via the interface provided by module Taxonomy Manager; two new goals are being added under category "frequent". 


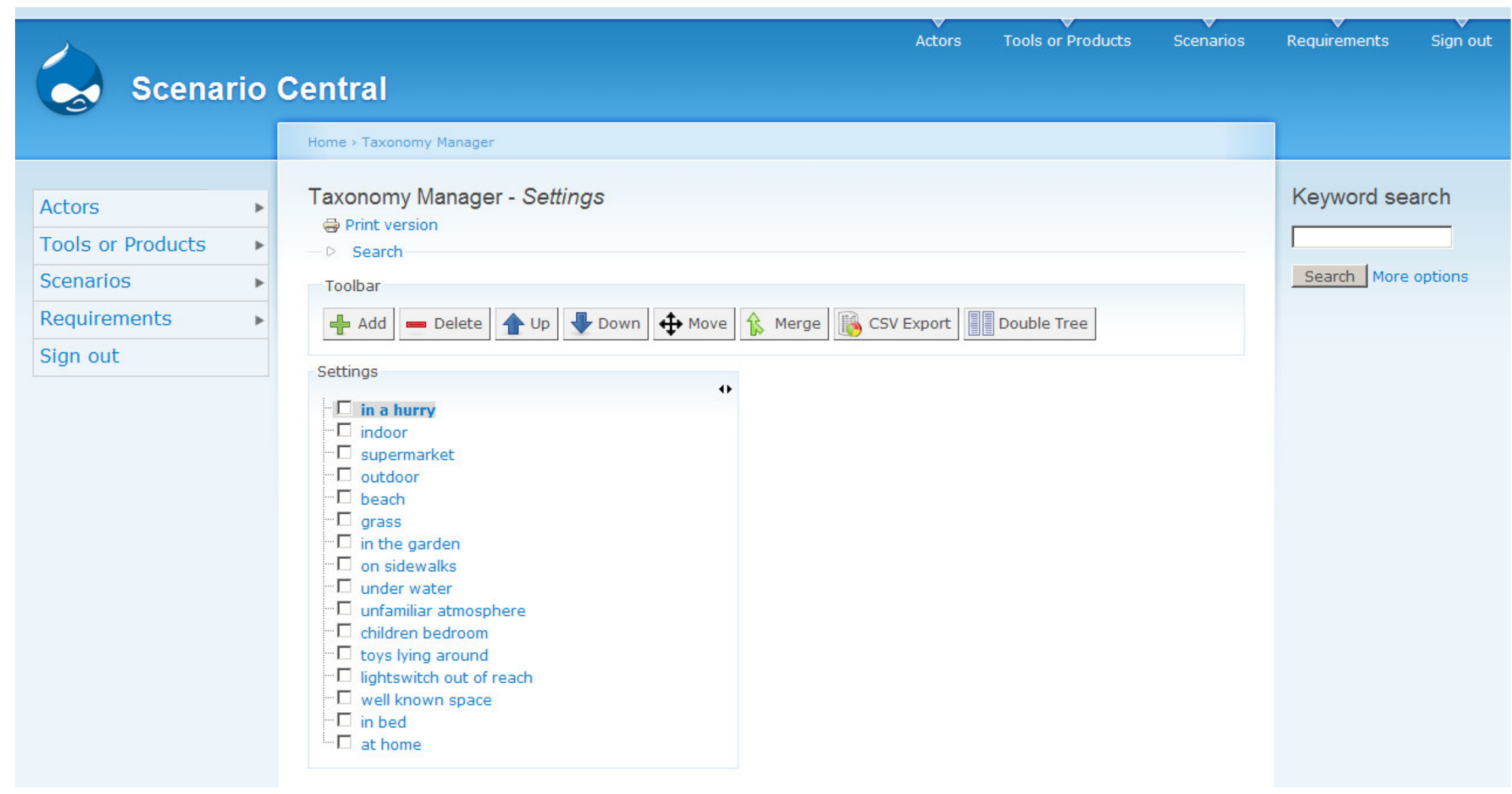

Figure 8.12: Similar to actor goals, settings can be added/edited using the same interface; although the example shows no hieararchy, depending on the specific need of the organization settings can also be grouped like actor goals. 


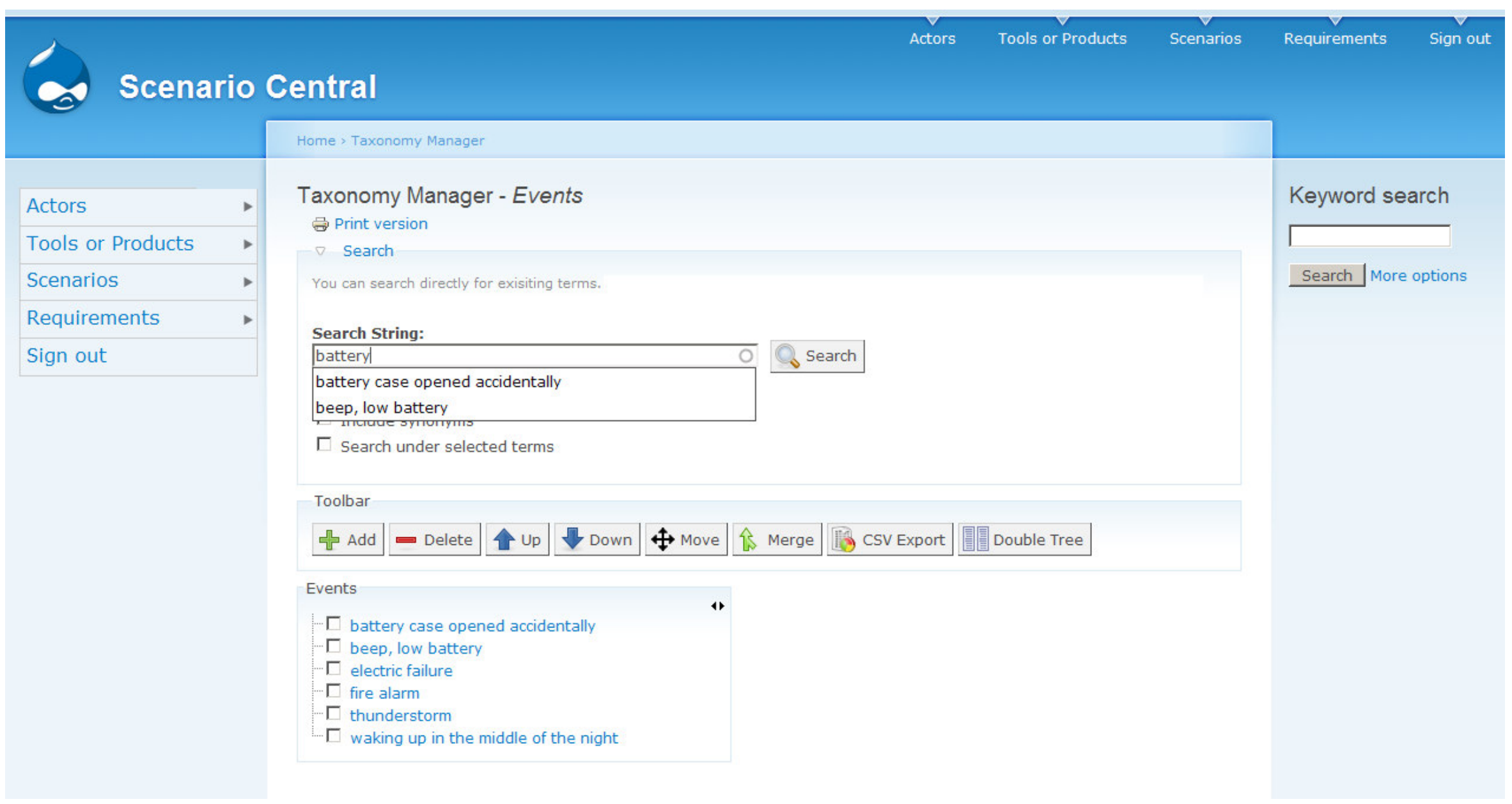

Figure 8.13: Events can be managed using the same interface; the interface allows filtering of terms as shown in the search box. 


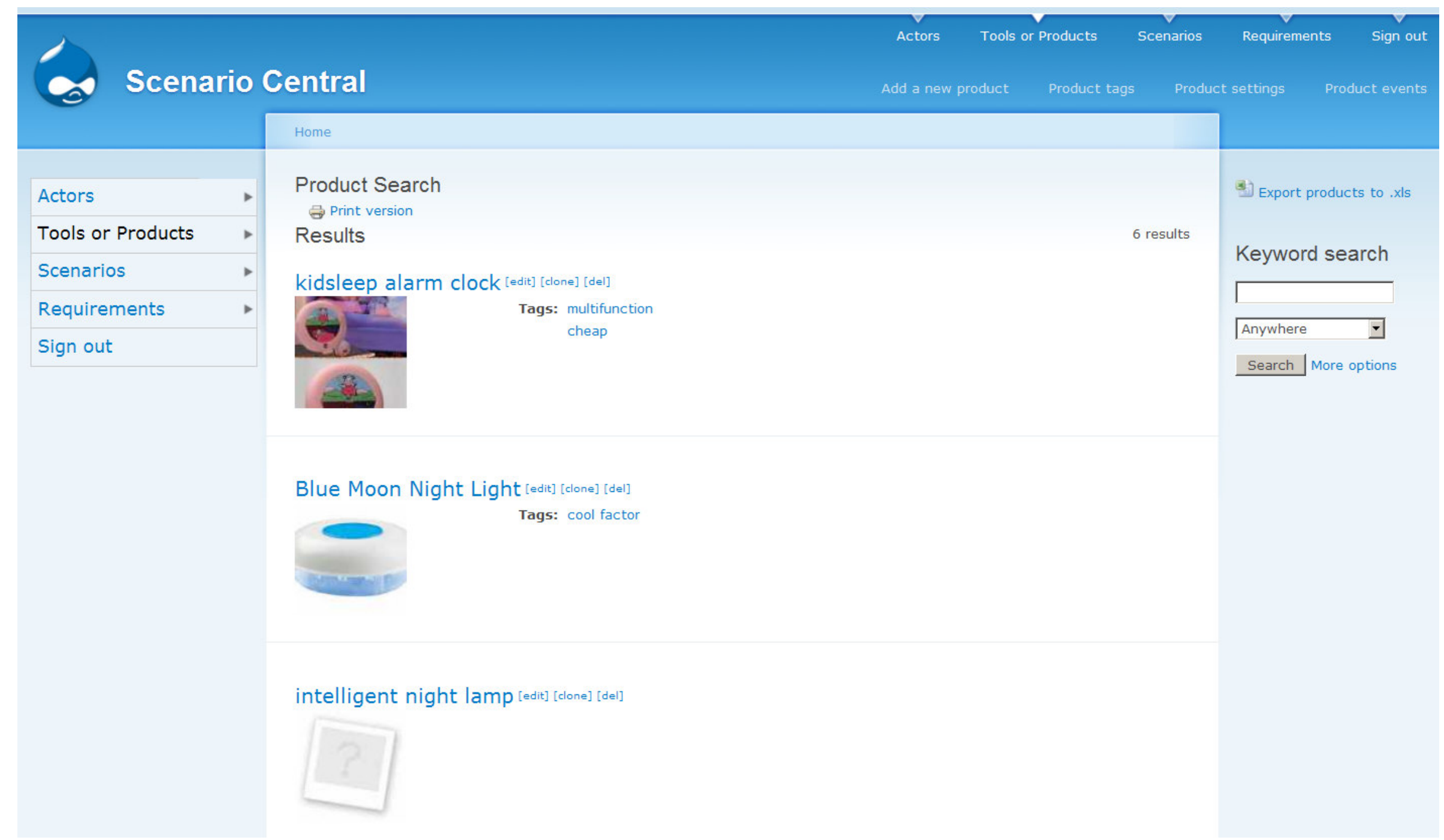

Figure 8.14: Displaying the list of products. 


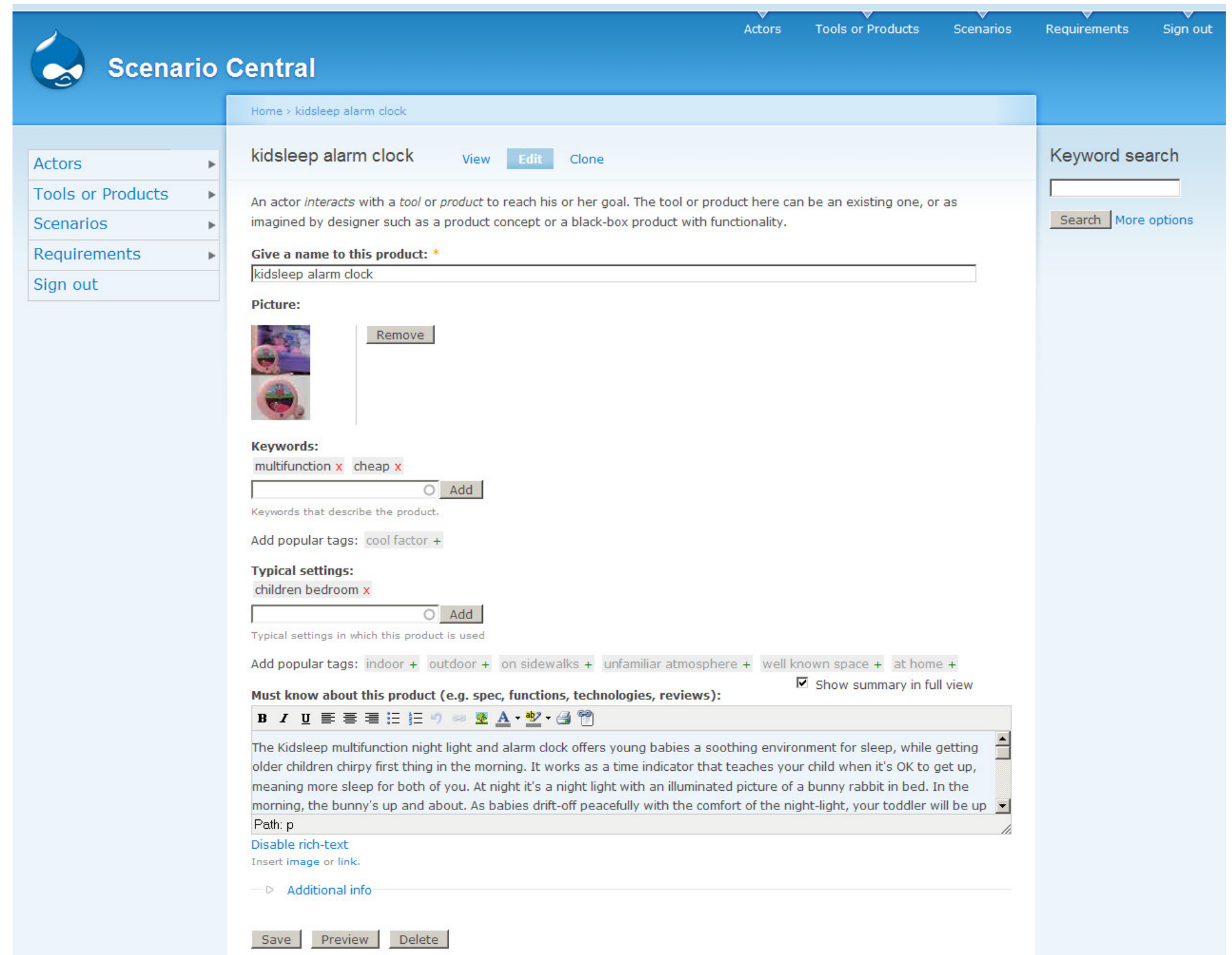

Figure 8.15: An overview of editing/creating a product profile; similar to actor profile, the link "Additional info" can be expanded to attach external documents. 


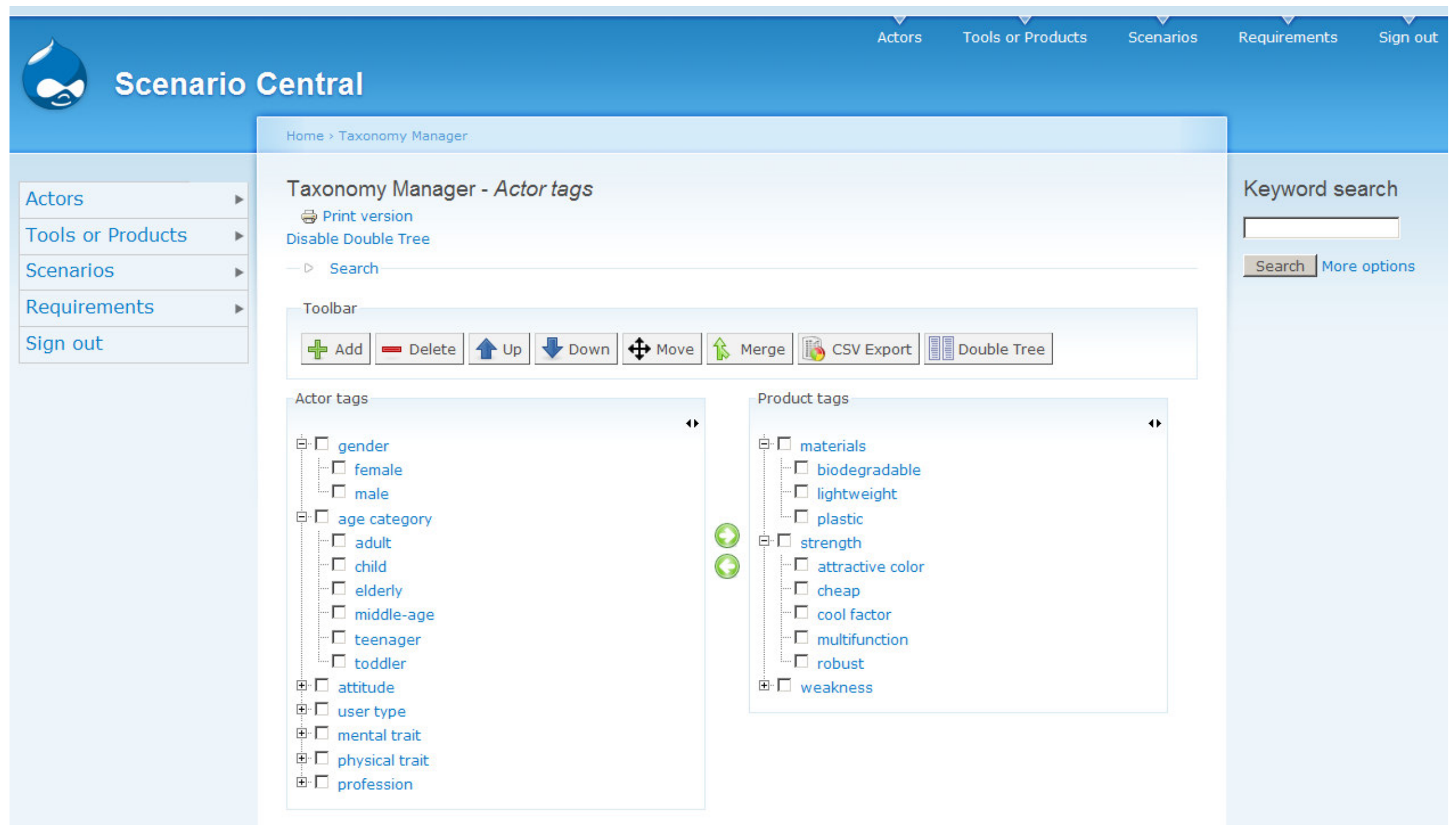

Figure 8.16: Both actor and product profiles contain keywords (tags), which as taxonomy terms can be managed in a similar manner to actor goals, settings and 


\subsection{Use case: "Create scenario"}

The steps for creating a scenario have been verified in the earlier evaluations. As an additional function, the designers wish to be able to allow the users to confirm the scenarios. The use case "confirm scenario" is therefore recognized in the use case diagram (Figure 8.17). In practice, the use case does not actually need a new function, as it concerns the designer's actual action in showing or discussing scenarios to/with users. Therefore there is no additional function to be addressed in the development.

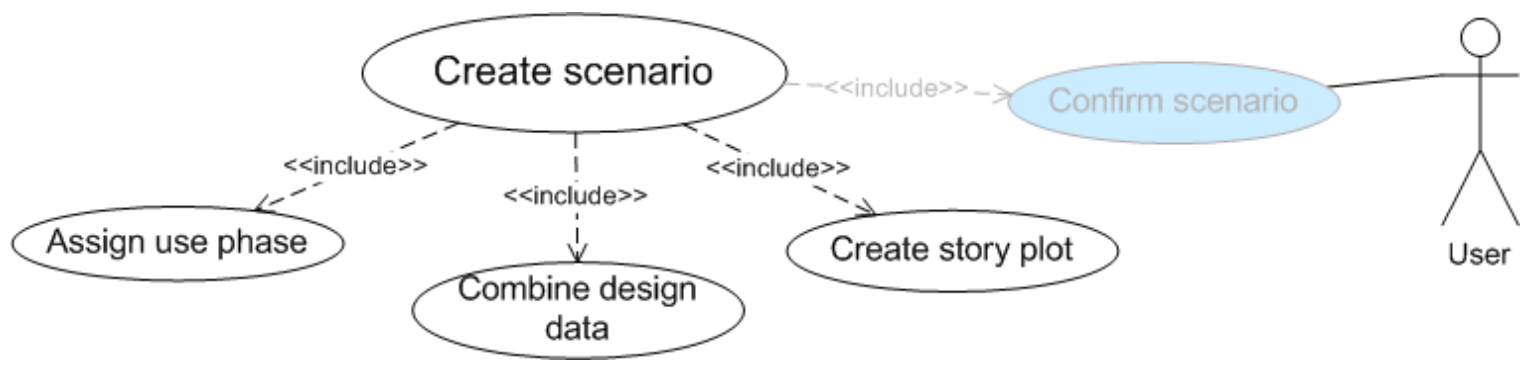

Figure 8.17: The implemented functions for use case "create scenario" in the final prototype; the greyed out use case "confirm scenario" is intentionally excluded.

Figure 8.18 to Figure 8.23 show the various parts of the final prototype that provide or support the functions related to creating scenarios. 


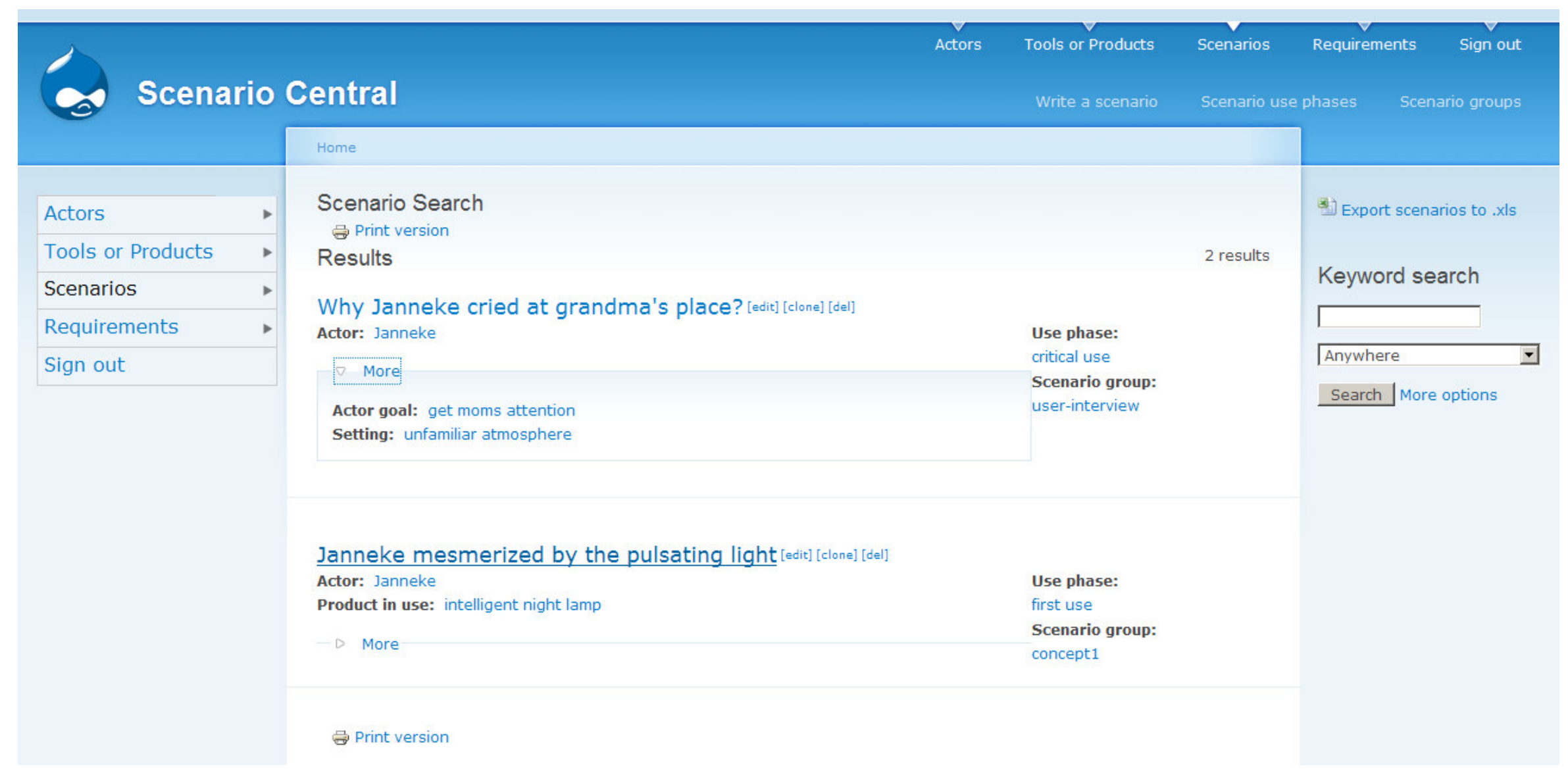

Figure 8.18: Displaying the list of scenarios; a possibility to see extra details of a scenario by expanding the link "More". 


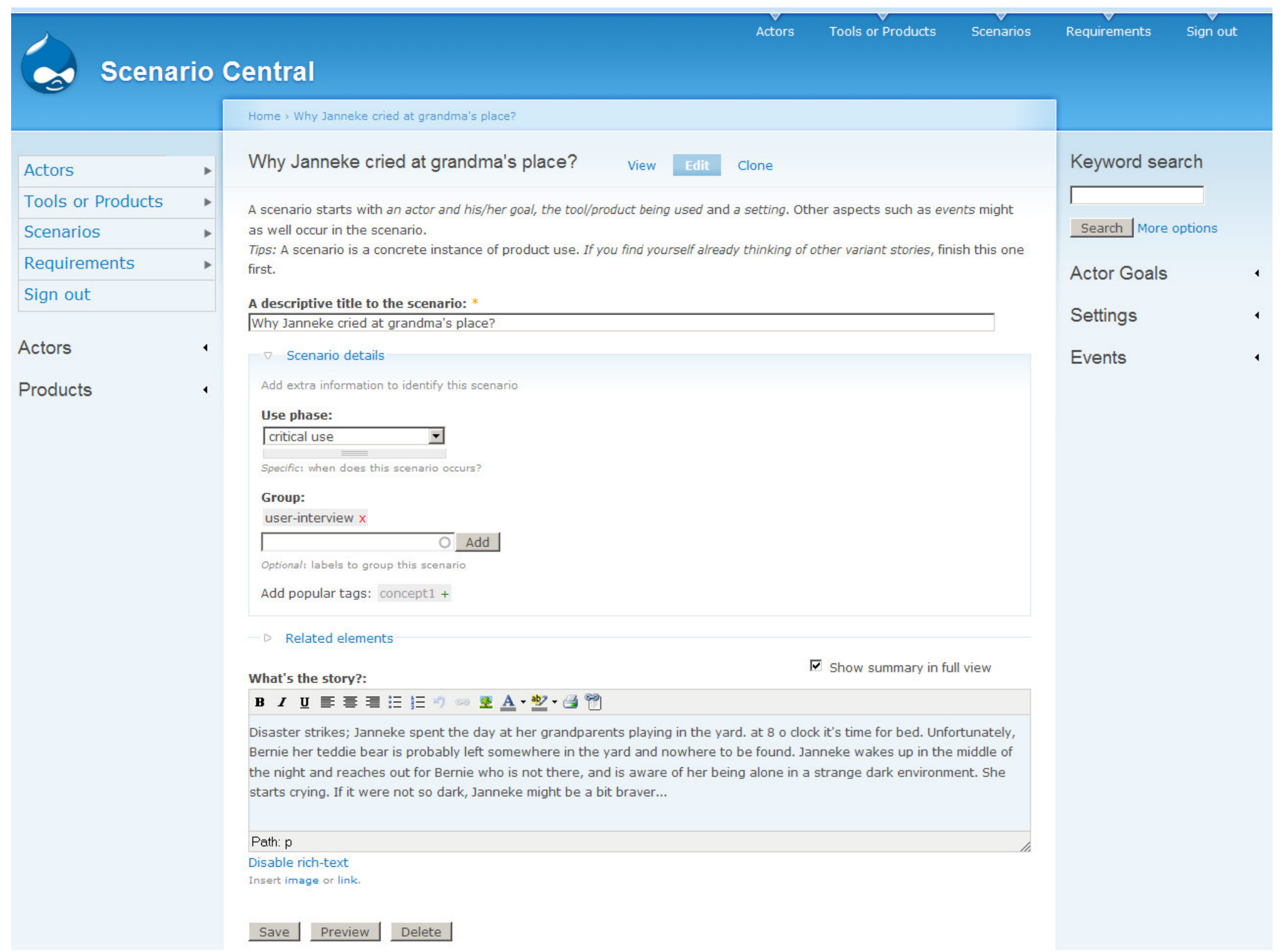

Figure 8.19: An overview of editing/creating a scenario; the links "Scenario details" and "Related elements" can be expanded to add extra information. 


\section{Related elements}

Refer to other documented elements as necessary

\section{Actor:}

Janneke [nid:89]

Who's the actor of this scenario?

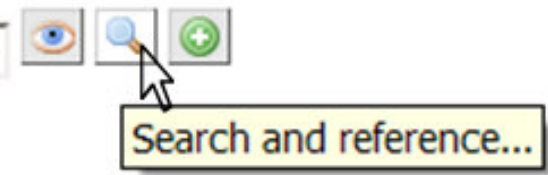

Actor goal: *

\begin{tabular}{|c|c|c|}
\hline frequent (4) & $\nabla$ & get moms attention (2) \\
\hline
\end{tabular}

What is THE actor's goal? One per scenario.

\section{Product in use:}

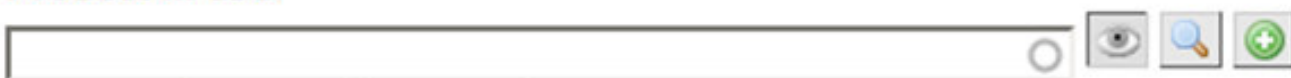

Is any product or tool being used?

\section{Setting:}

unfamiliar atmosphere $\mathrm{x}$

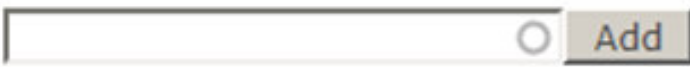

Select situations that apply in this scenario

Add popular tags: indoor + outdoor + on sidewalks + children bedroom + well known space + at home +

Started with an event?:

<none $>\quad+$

The event that might have triggered this scenario 
Actor:

Search and reference

Create and reference

Currently selected Actor: Janneke [nid:89]

Actor name

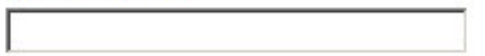

Keyword

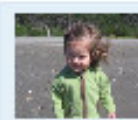

Janneke

Keyword: female, main persona,

toddler

Author: admin

Last update: Thu, 05/08/10

$16: 23$

h)

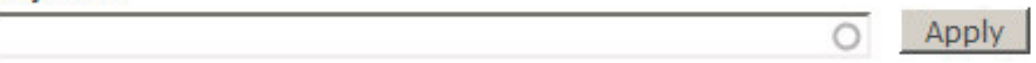

Figure 8.21: Search and reference an actor (from scenario creation) displays the searchable list of actors; the same applies for search and reference a product.

Keyword: adult, Blind, Diabetic,

male, Obese

Author: tester2

Last update: Wed, 20/01/10

$11: 09$

$[+]$ Feedback 


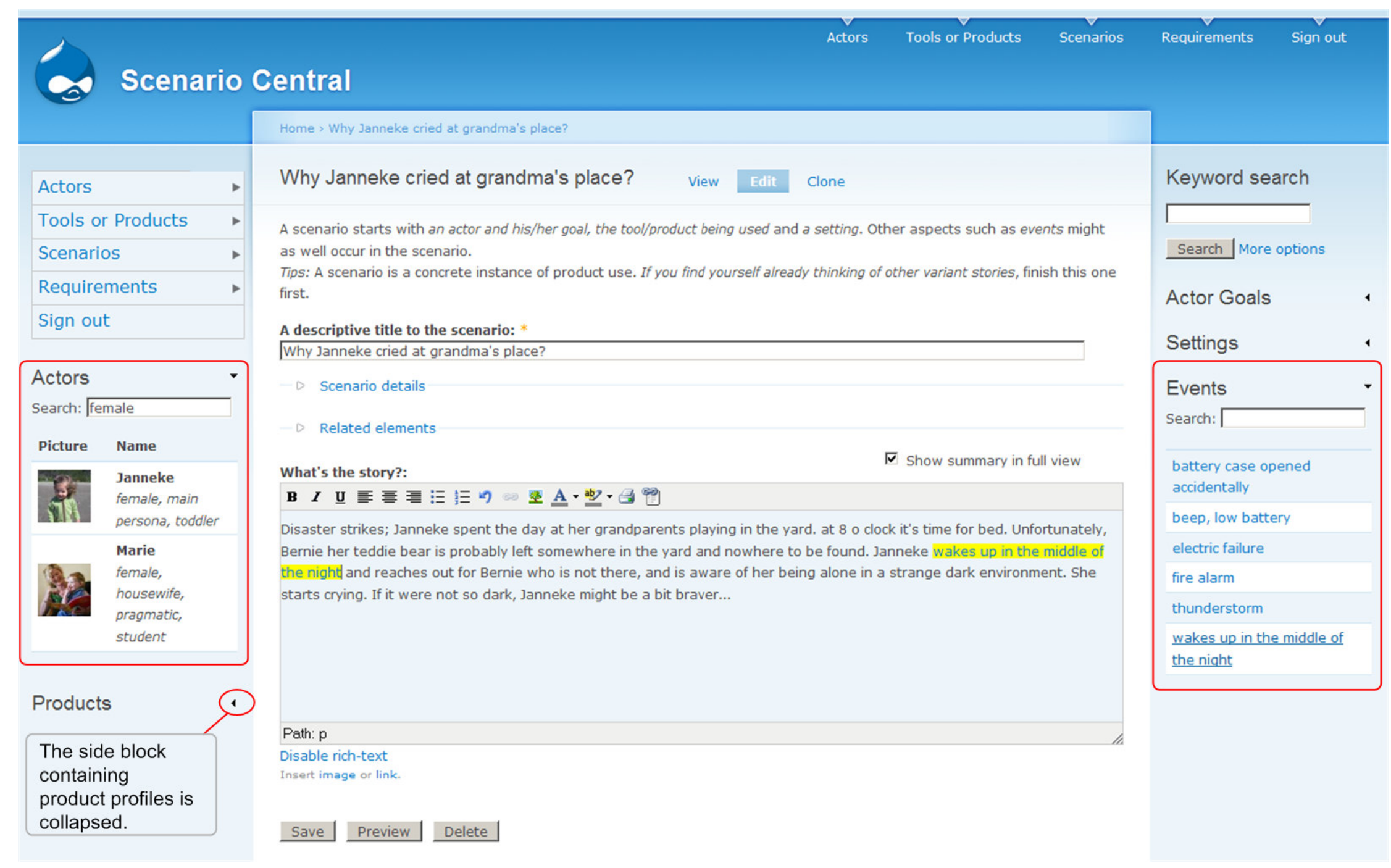

Figure 8.22: Editing/creating a scenario is assisted by collapsible side blocks that contain all elements that could possibly be used in the scenario; the 


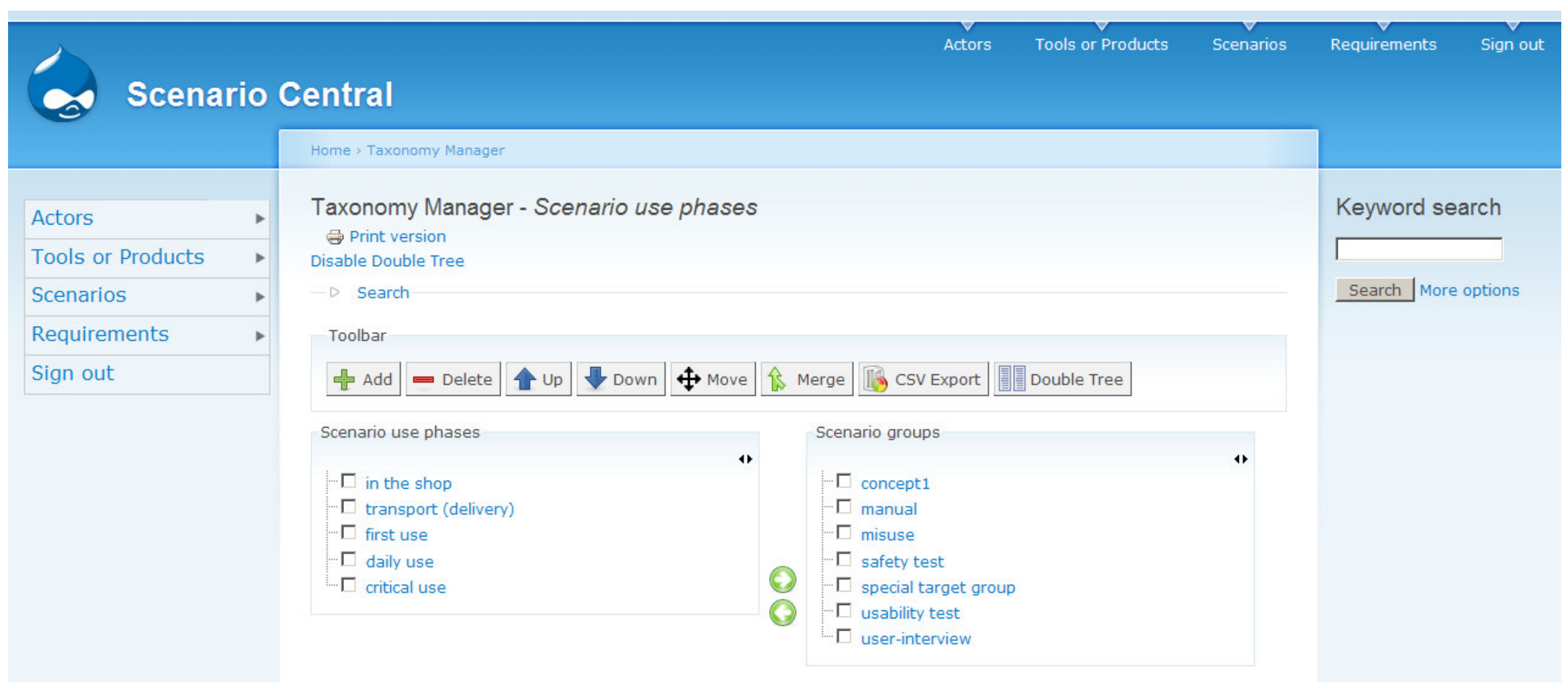

Figure 8.23: A scenario can be identified and grouped by its use phase (in which it happens) and/or custom group labels e.g. by purpose (for 'safety test'), source (from 'user-interview'), or relation with a particular solution (describing 'concept l'). 


\subsection{Use case: "Create requirement"}

The support tool accommodates the creation of requirements as a process inspired by other design information such as scenarios. It is possible to make explicit the interrelation between requirements and other information, for instance by referring to one or more scenarios as a rationale for a requirement. There are many types of requirements within complex product design. The support tool however does not aim to address the creation of all possible types. Furthermore, there already exist approaches and tools that specifically guide requirements engineering by addressing the creation and management of comprehensive, complete lists of requirements. The support tool will instead focus on the earlier, brainstorming phase, where its role is to inspire the identification and formulation of requirements. Such requirements are mainly related to the users and the use of the product, usually addressing usability issues, and therefore can be inspired by scenarios. In accordance with keeping it informal, simple and nonbureaucratic, the tool accommodates creating requirements, without requiring the designers to confirm them with the manager first. Figure 8.24 shows the simplified steps of creating requirements, without any confirmation step.

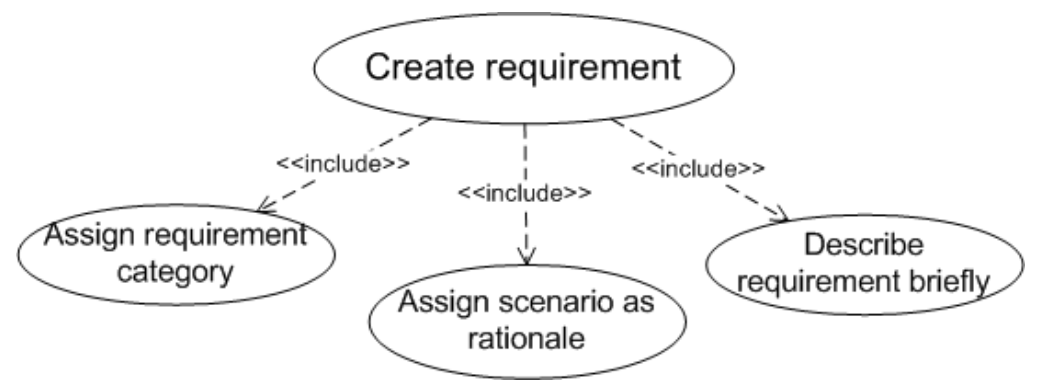

Figure 8.24: The simplified use case "create requirement" is implemented in its entirety.

Figure 8.25 to Figure 8.28 show how requirements can be suggested from other design information, and created in the support tool. 


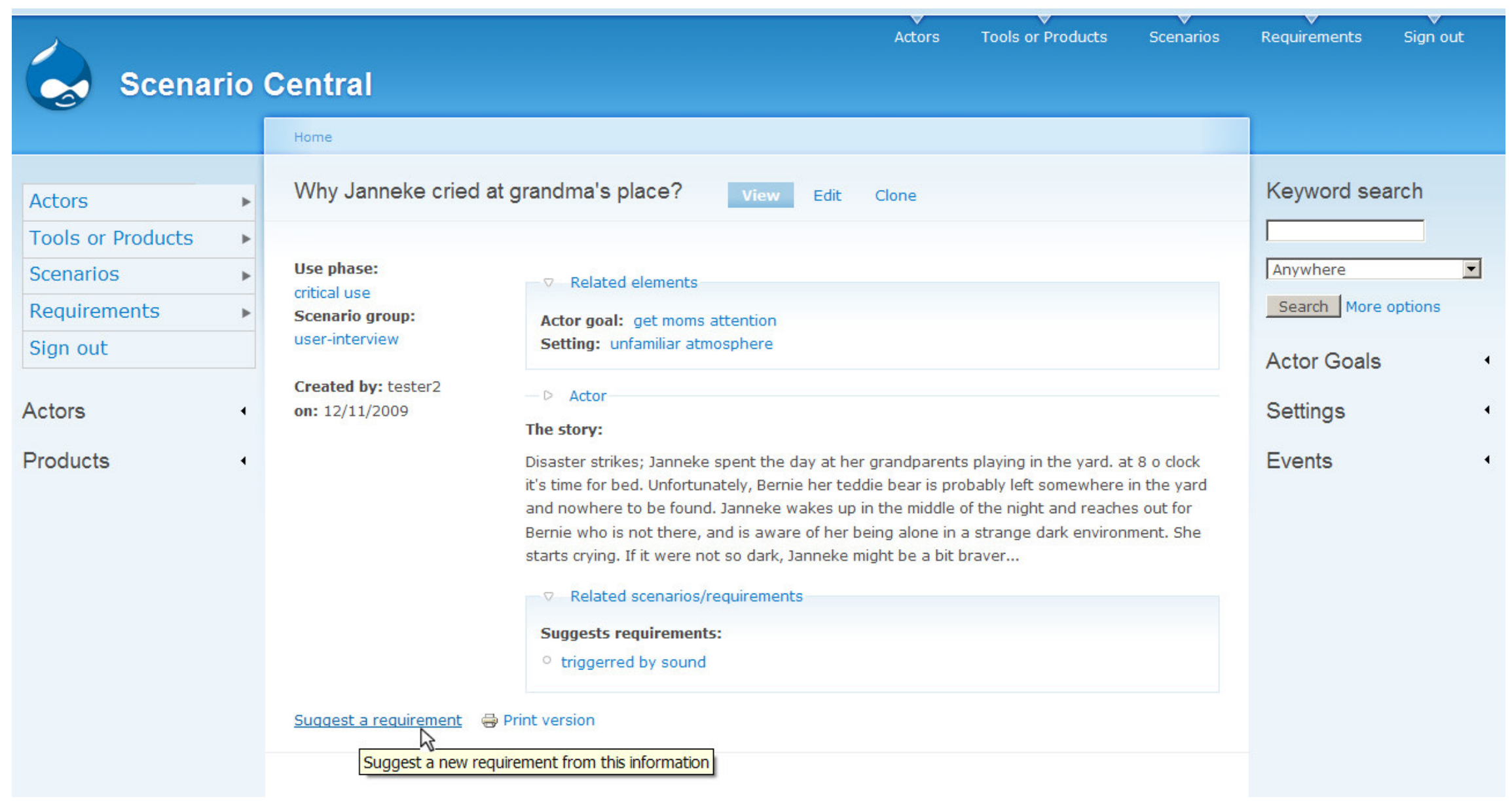

Figure 8.25: On viewing a scenario, a requirement can be suggested with the scenario as its inspiration by clicking on the link 'Suggest a requirement'. 


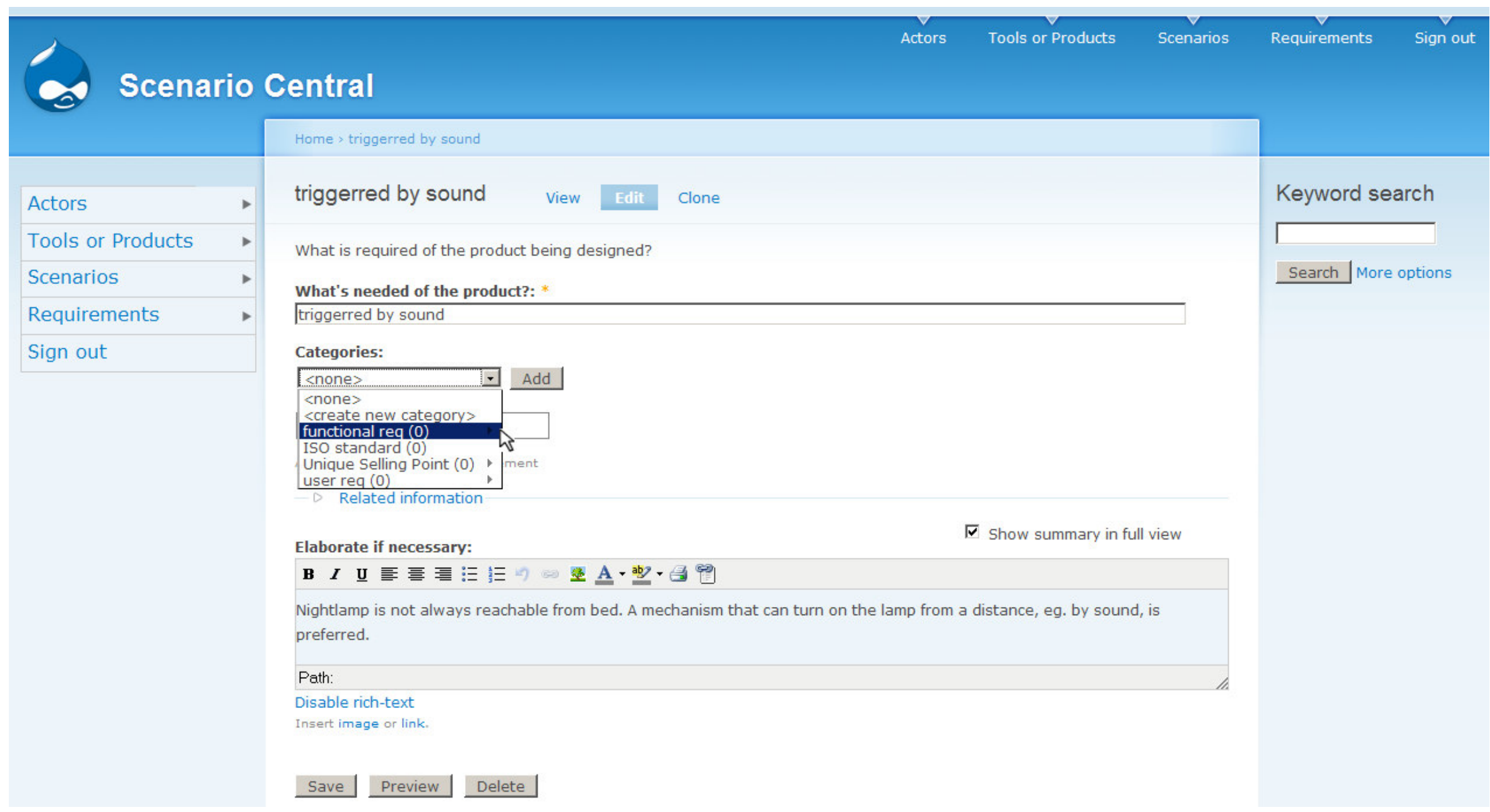




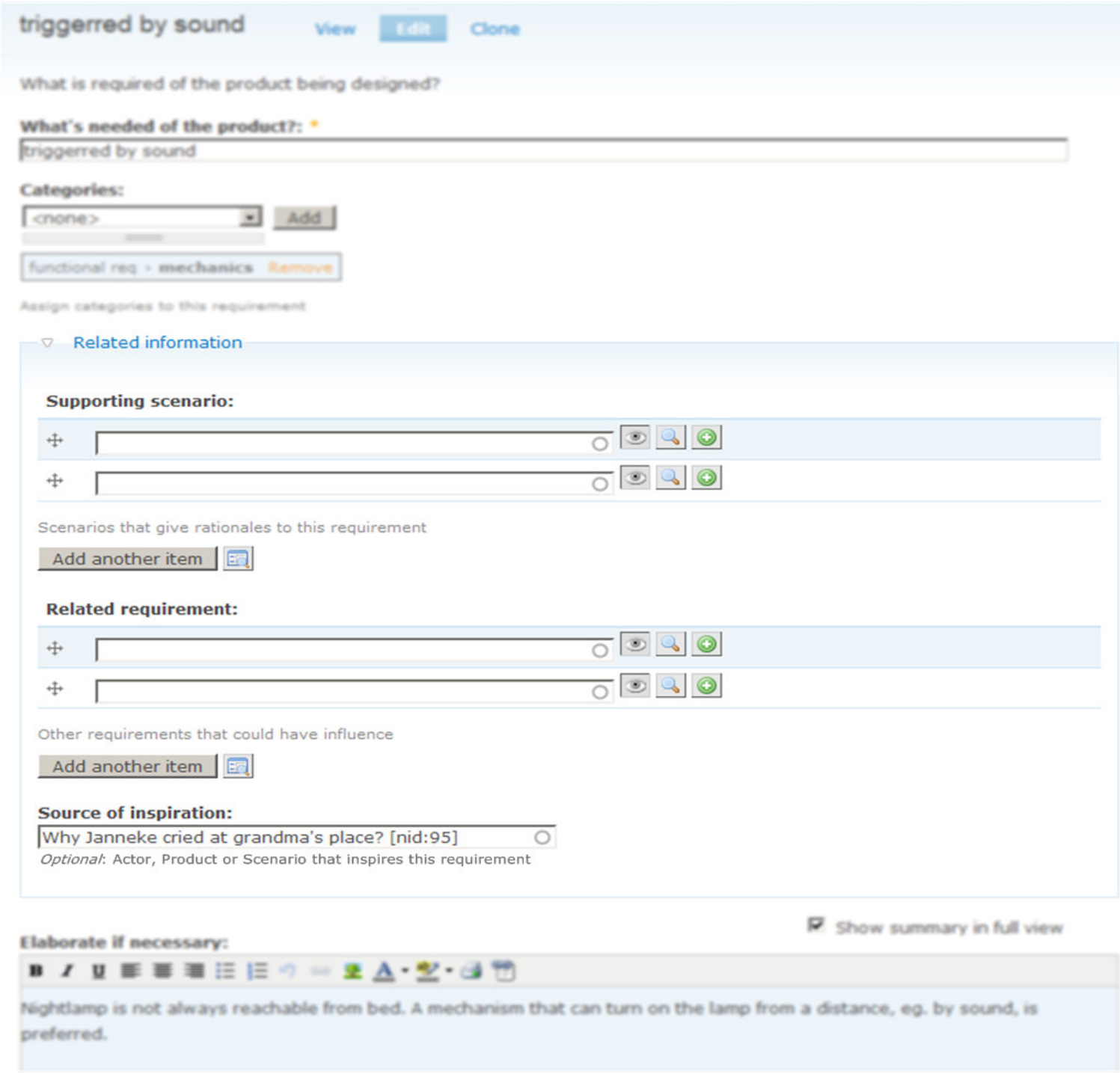

Figure 8.27: A requirement can be related with other information, i.e. scenarios or other requirements, and can have a source of inspiration (also see Figure 8.25). 


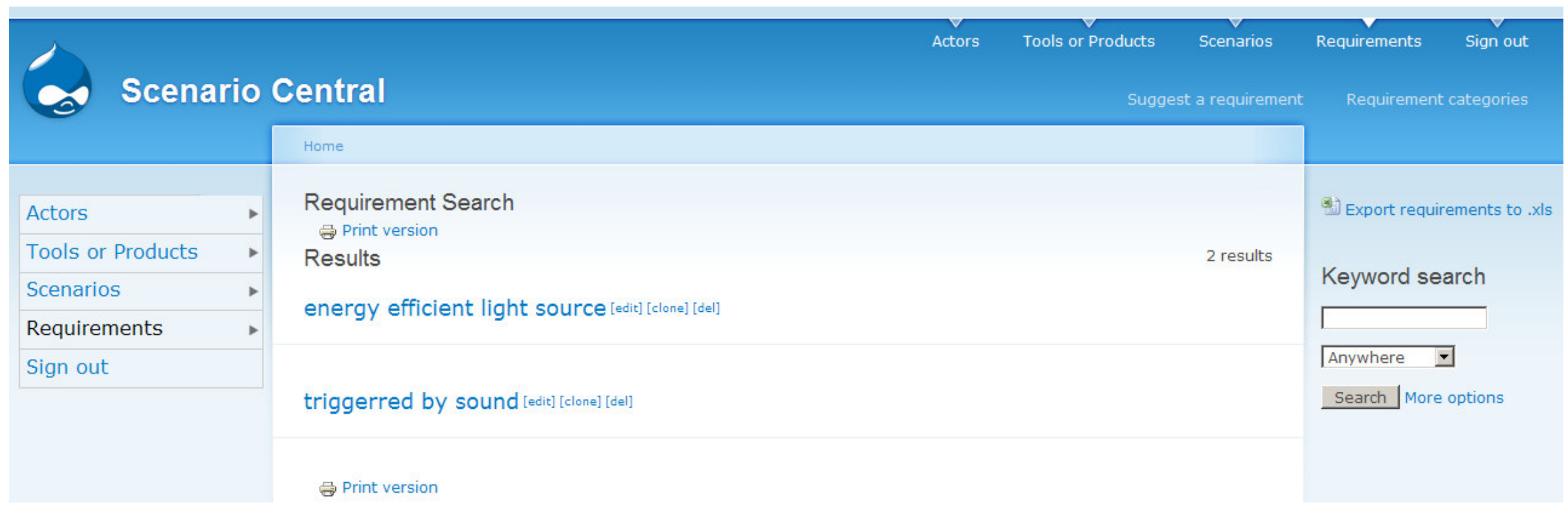

Figure 8.28: Displaying the list of requirements upon saving the new requirement. 


\subsection{Use case: "Use information"}

To use information effectively and efficiently, the support tool provides a possibility to search all the information using different criteria. Additionally, functionality to filter information is considered useful to limit the scope of information, e.g. to a particular project, particular tasks, or just personal interests. Such functionality will help the designers to avoid being overwhelmed by information and focus on the relevant, most important one. Due to time and resource constrain, however, it is not fully implemented in the prototype.

Another useful functionality concerns displaying information in a more visual way to improve the overview of all available information. However, since it deals with the representation of information, which is outside the scope of this research, the use case "display information" is not addressed in the prototype development.

To support the activities of using information, it should be possible to manage this information at least with the basic operations of update, delete and copy. The use case "manage information" does not concern a particular function, as it is integrated in all information within the support tool. The previous sections have shown access to update, delete and copy operations in different examples (e.g. in Figure 8.5). The final prototype has implemented the search and management functionality, as illustrated in Figure 8.29.

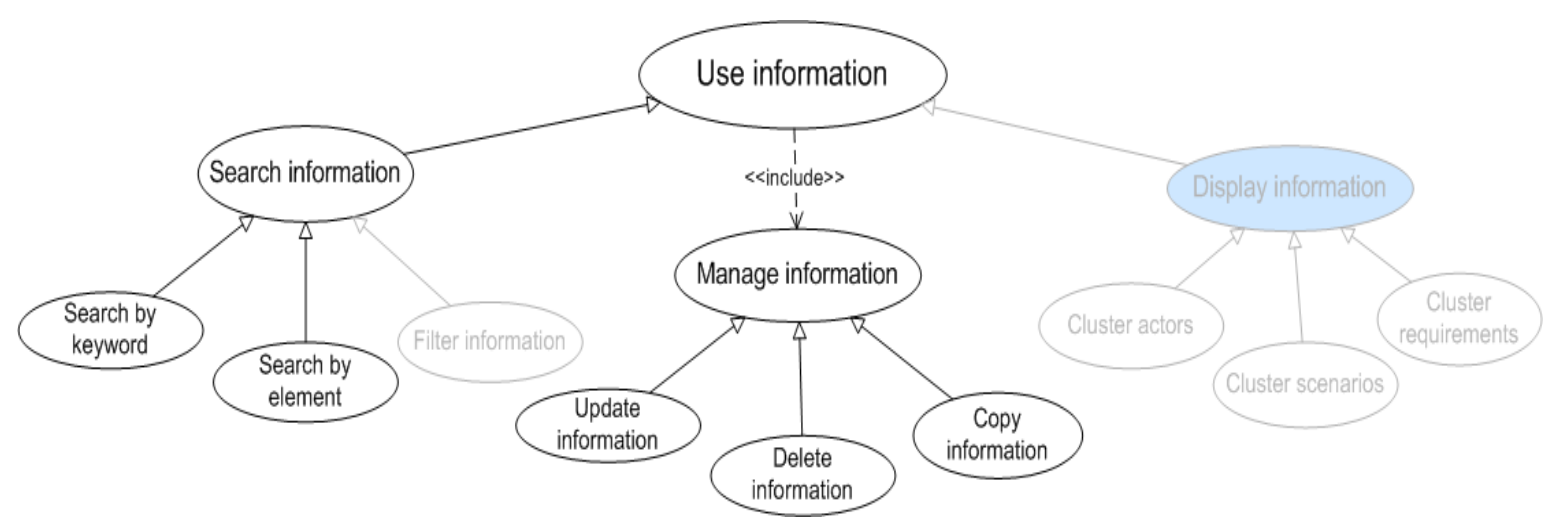

Figure 8.29: The implemented functions for use case "use information" in the final prototype; the greyed out use cases are intentionally excluded.

Figure 8.30 to Figure 8.33 show several examples on searching information within the support tool. 


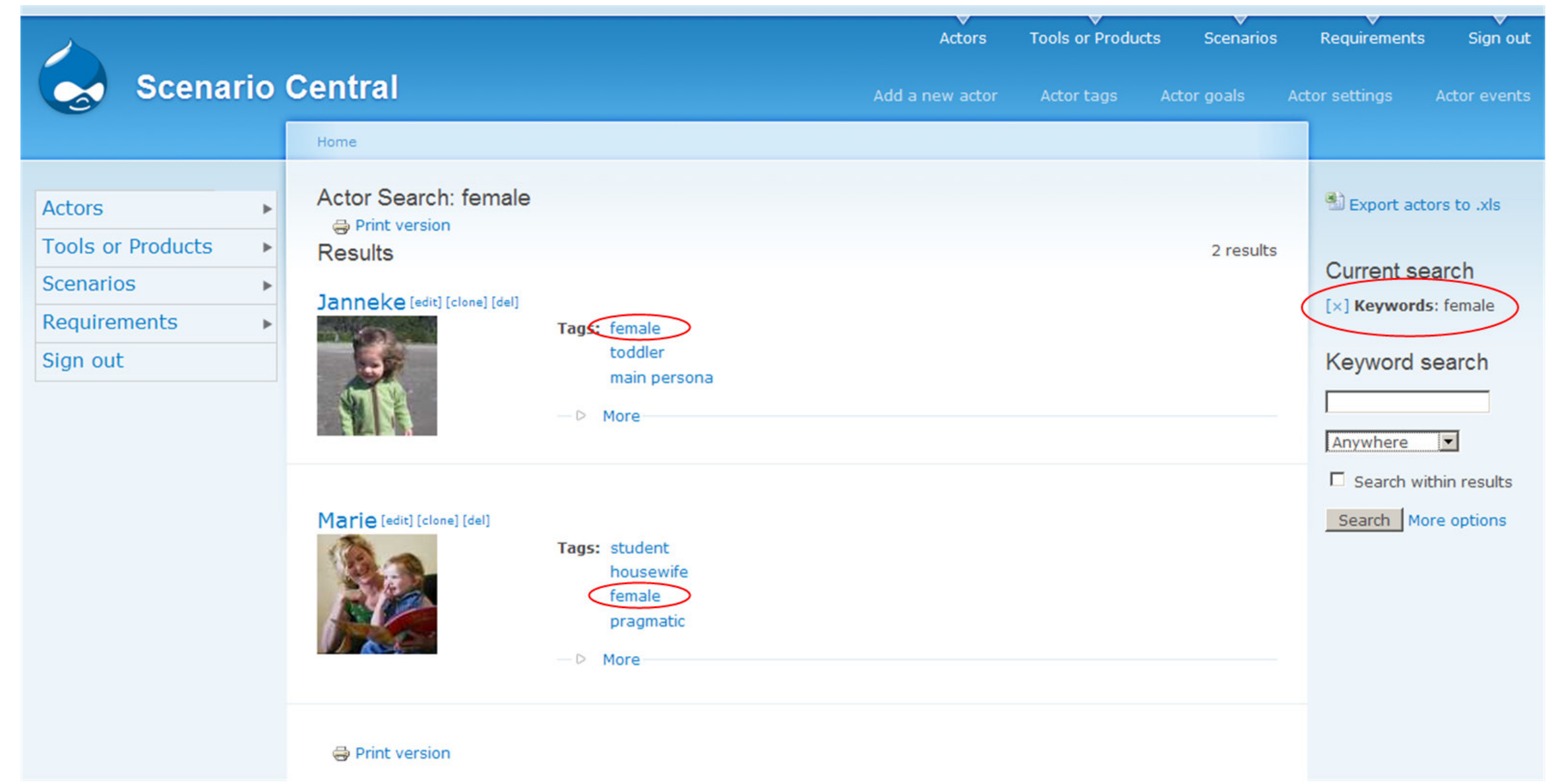

Figure 8.30: The list of actors (and others) can be searched by keyword; the example shows a search on actors containing the keyword 'female'. 


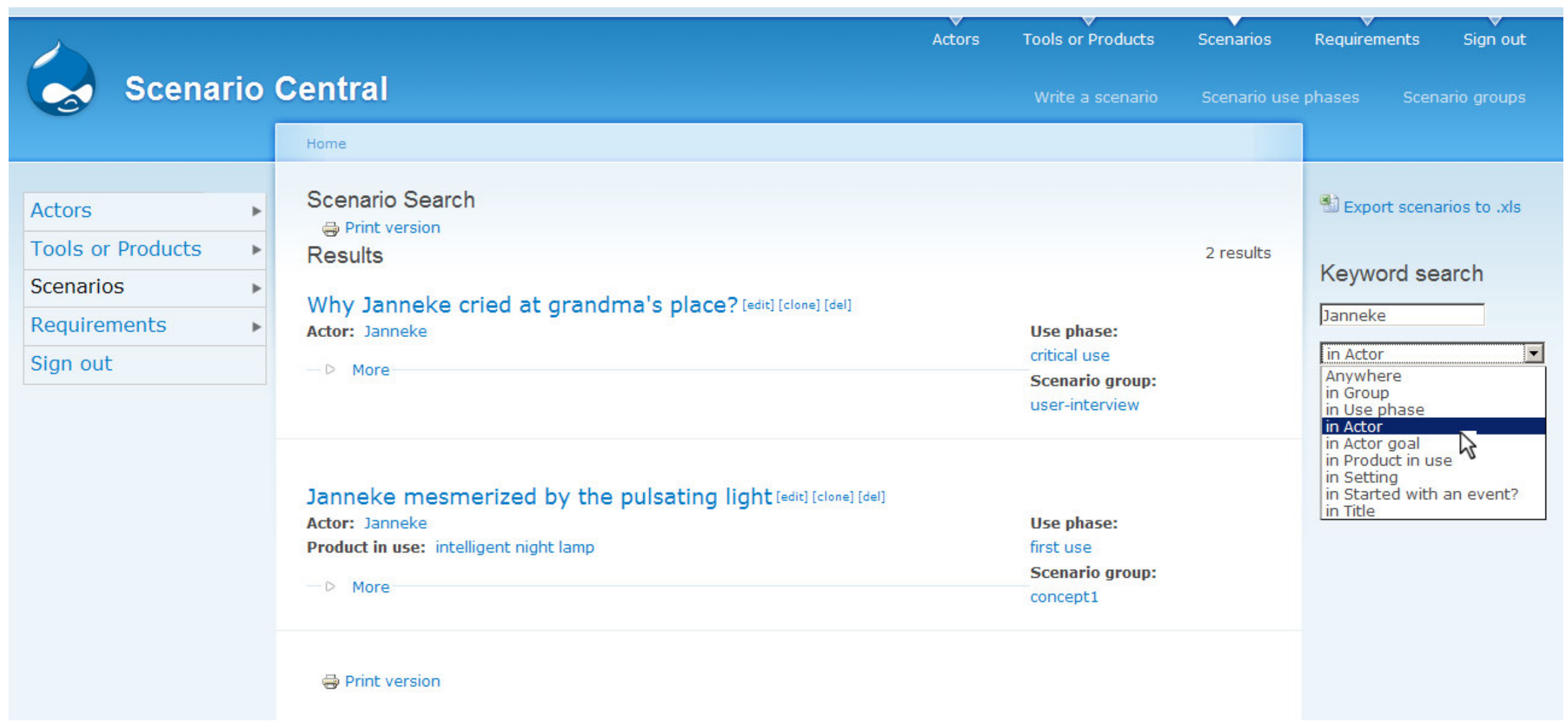

Figure 8.31: The list of scenarios can be searched by its element; the example shows a search on scenarios that involve actor 'Janneke'. 


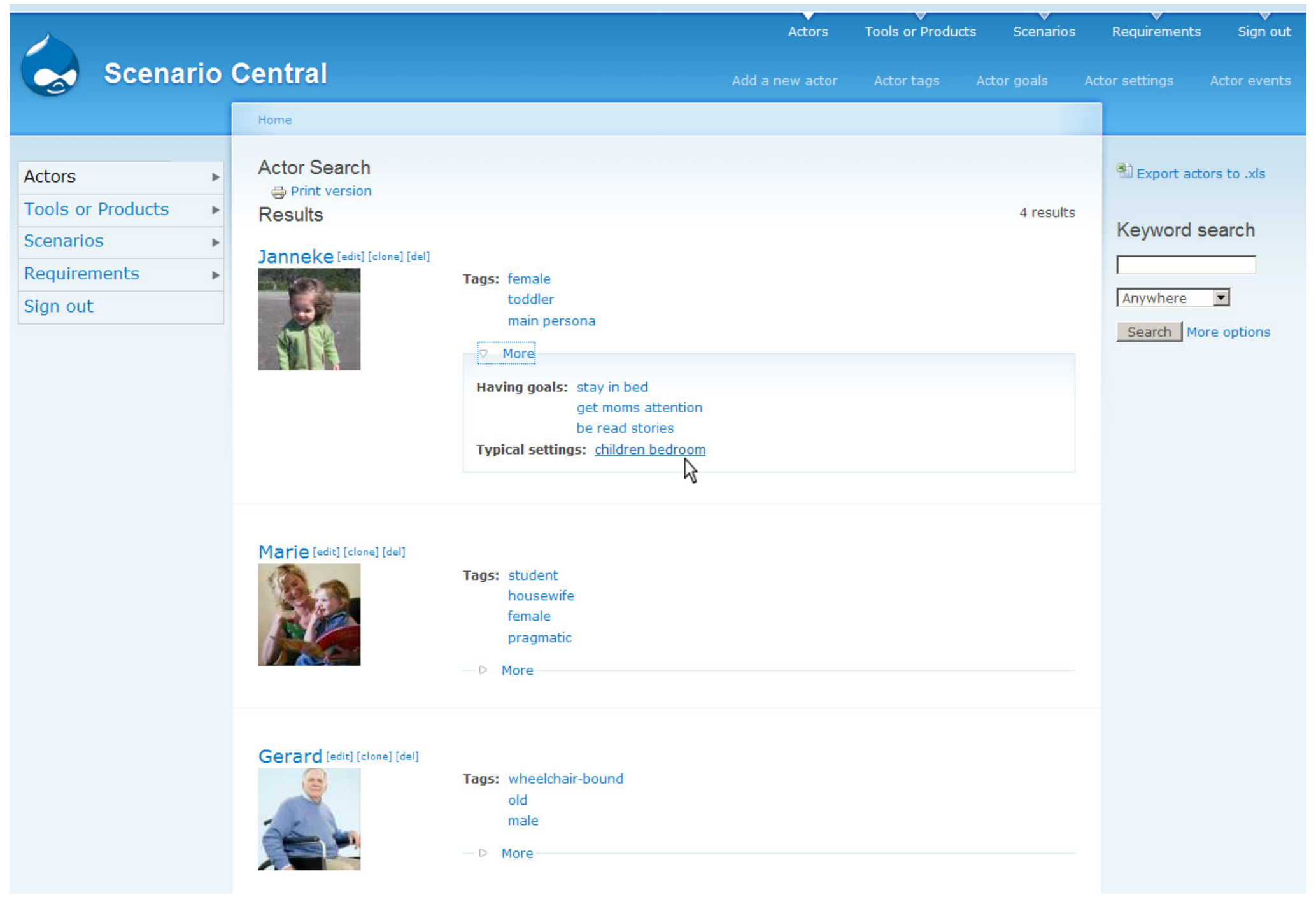

Figure 8.32: Another manner to search is by clicking on a specific element (in the example, setting 'children bedroom'); the result is shown in Figure 8.33. 


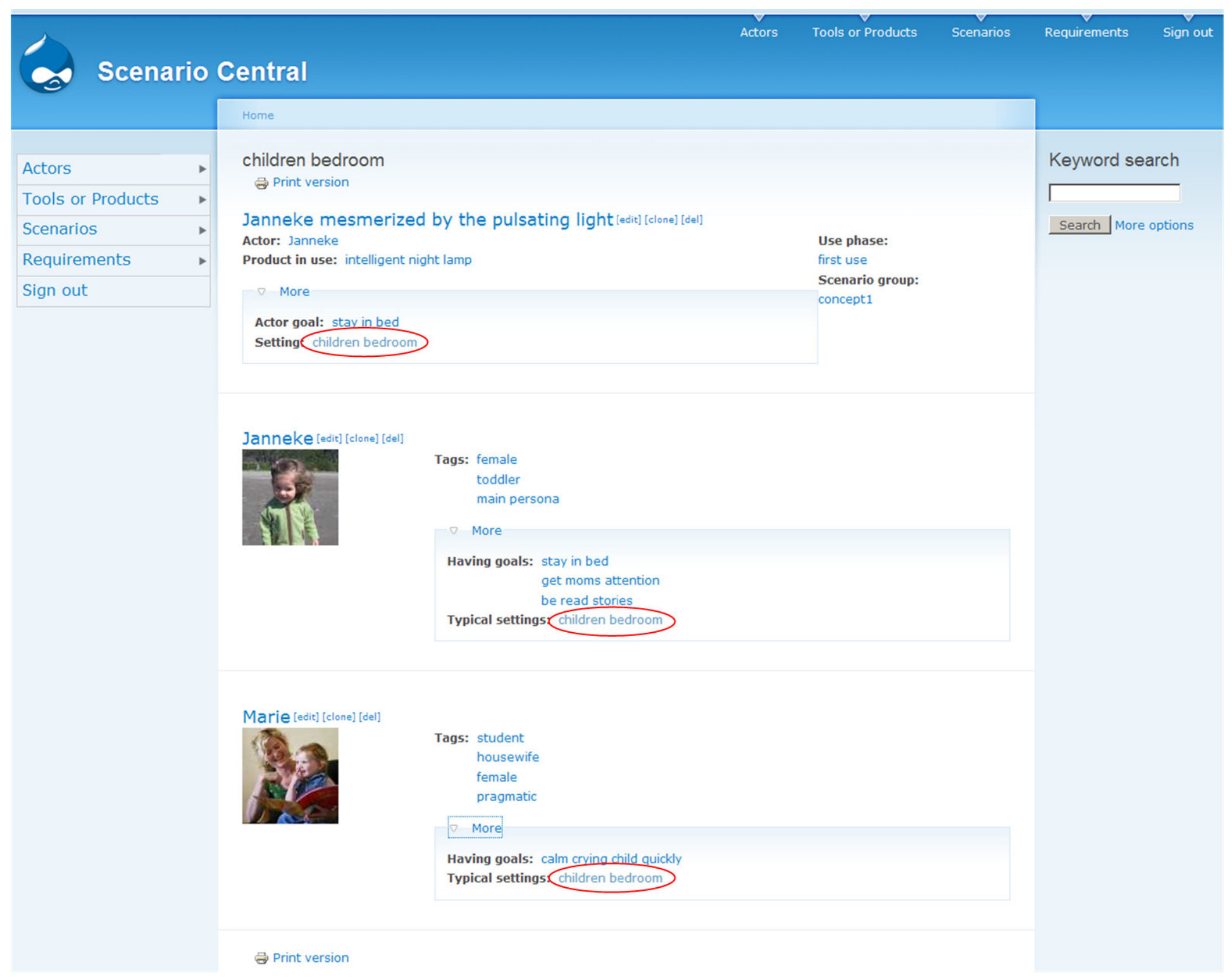

Figure 8.33: A search on specific element (setting 'children bedroom') displays all information that contains the element (see also Figure 8.32). 


\subsection{Use case: "Deliver information"}

A very practice-oriented use case "deliver information" has been discussed in the earlier evaluations. Its implementation would be specific to the particular organization, and therefore has not been addressed in the development. Different design practices utilize varied approaches, methods, or tools which could make use of the information within the support tool. To ensure effectiveness and efficiency, the support tool would need to adjust the information it delivers to the specific formats required by these approaches, methods or tools. Furthermore, the involvement of various stakeholders in the design project might also require different formats of reports from the tool. To consider these variables during the prototype development was simply not feasible. Nevertheless, the final prototype implements basic printing functionality to demonstrate the possibility to deliver information, as shown in Figure 8.34.

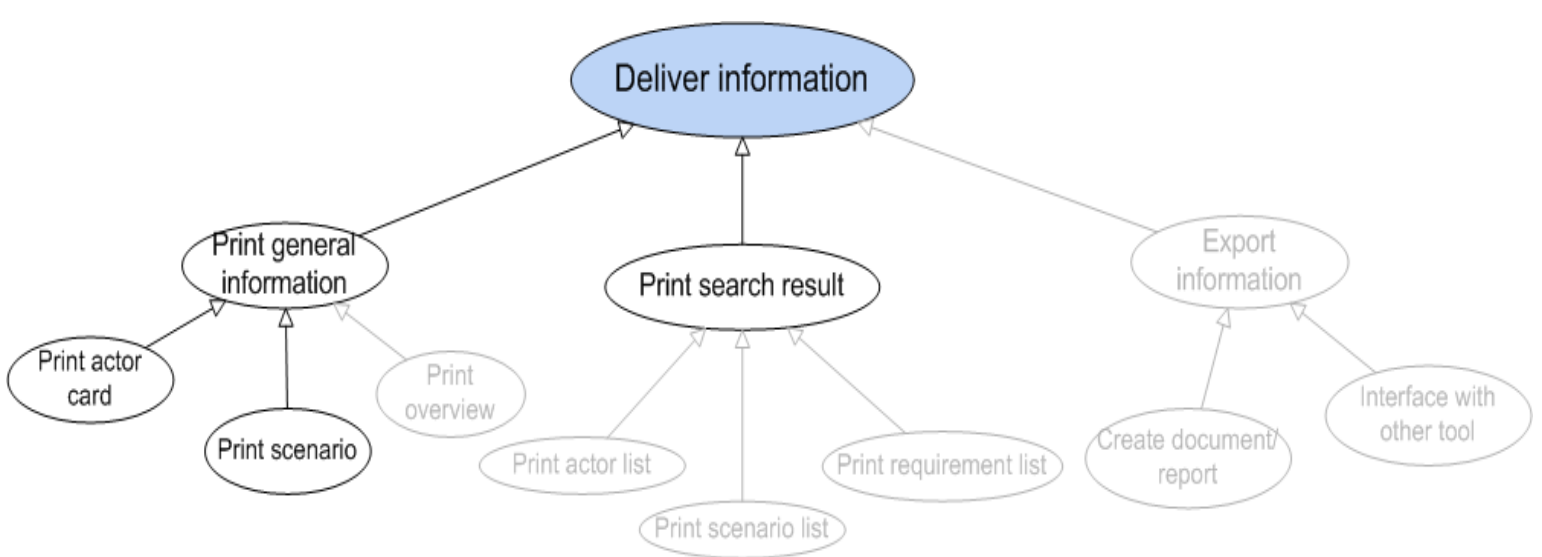

Figure 8.34: The implemented functions for use case "deliver information" in the final prototype; the greyed out use cases are intentionally excluded.

Figure 8.35 to Figure 8.37 show some examples related to creating printed output using the support tool. 


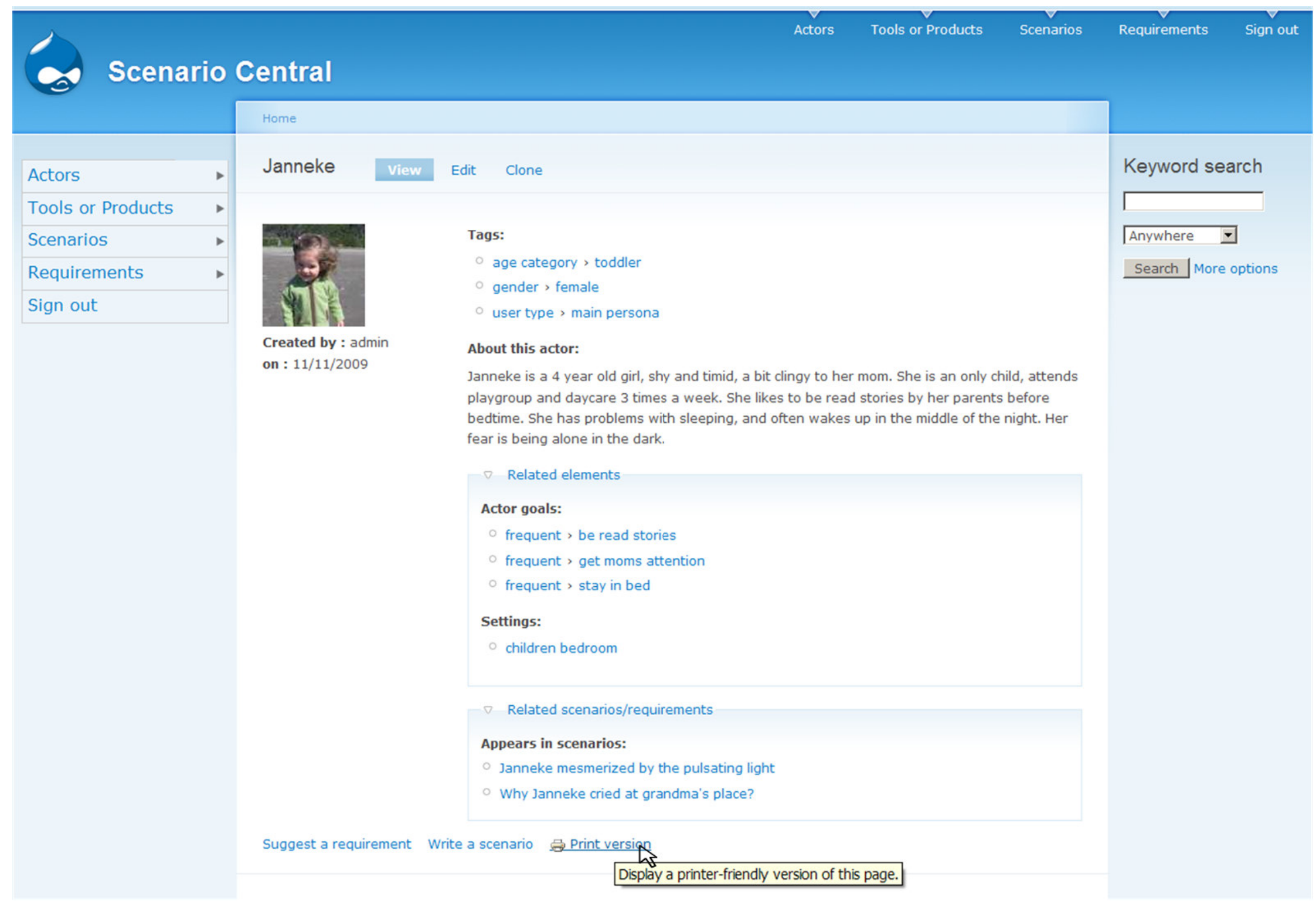




\section{Janneke}

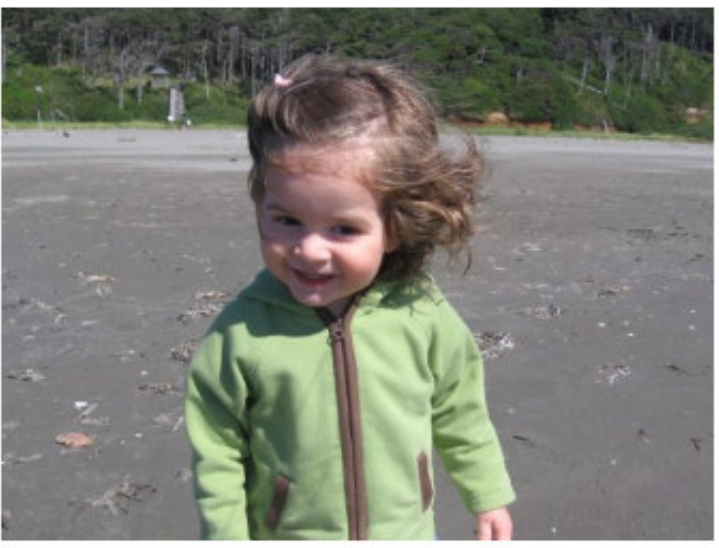

Tags:

- age category , toddler

- gender , female

- user type s main persona

-Related elements

Actor goals:

- frequent , be read stories

- frequent > get moms attention

- frequent, stay in bed

Settings:

- children bedroom

Janneke is a 4 year old girl, shy and timid, a bit clingy to her mom. She is an only child, attends playgroup and daycare 3 times a week. She likes to be read stories by her parents before bedtime. She has problems with sleeping, and often wakes up in the middle of the night. Her fear is being alone in the dark.

Source URL (retrieved on 05/08/10 19:48): http://ctwopms002.ctw.utwente.nl/finalsbdtool/actor/janneke

Figure 8.36: An example of a printer-friendly version of an actor profile 'Janneke'. 


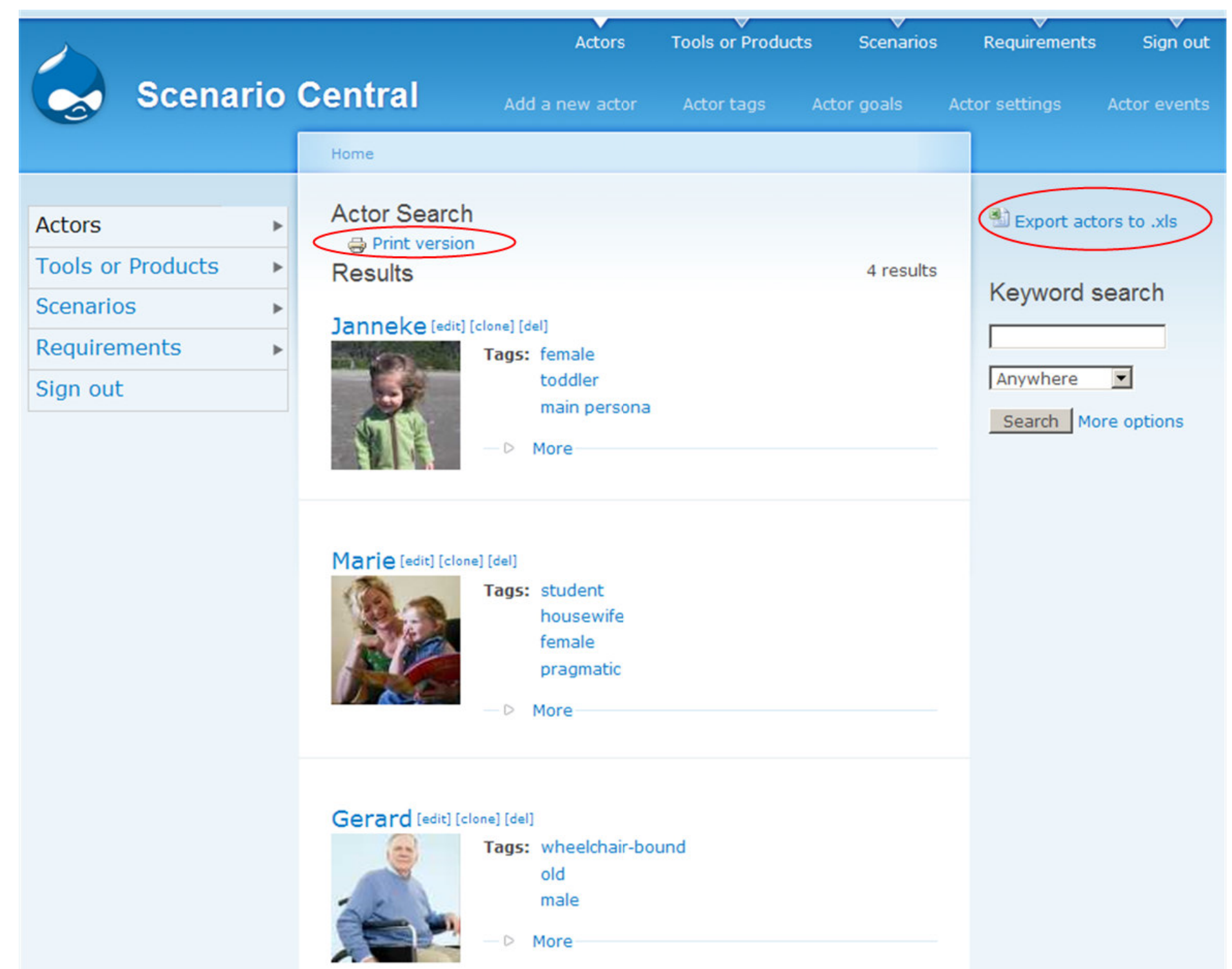

Figure 8.37: In any search result, the links to generate printer-friendly version and export to another format are present; "export to .xls" is specific to Indes and only serves as an example in the prototype. 


\subsection{Conclusion}

This chapter has described the final prototype of the support tool in a great detail. The main tool functionality has been consistently implemented in the final prototype. However, not all functionality could be realized for two reasons. Firstly, its implementation would be dependent on the specific practice of an organization. Since the support tool is developed to guide a general approach in creating, using and organizing scenarios, and not to cater the specific requests of any design practice, any organization dependent functionality is not implemented in the prototype. Secondly, there are limitations in time and/or means (i.e. Drupal) to implement the functionality. Some adjustments have been made to translate the interfaces and interactions as proposed in the conceptual design (Chapter 5) into what was feasible within the development timeframe and platform. Despite these limitations and adjustments, the capability of the final prototype has not been affected. It serves sufficiently to demonstrate the functionality and use of the support tool.

The final prototype will once more be evaluated with design practitioners. To find out the values the support tool can bring in the design industry at large, the evaluation will involve two companies with different characteristics to represent the diversity of our target users. The next chapter will describe the final evaluation and the findings. 



\section{Final Evaluation}

The final evaluation aims to evaluate whether the final prototype demonstrates the correct implementation of the identified functionality. The functionality has been developed with the practice of a small/medium-sized design agency as a reference. To explore additional contexts in which the support tool can be useful, the evaluation scope is broadened by involving a large-scale design company. As a large company, Philips is selected because it has characteristics that complement those of Indes. By approaching Philips, this research aims to find relevance for the support tool in another context than small/medium-sized design agencies.

Philips is a long-standing Dutch company and is omnipresent in the various aspects of everyday life mainly with their consumer electronic products. Both Indes and Philips have a good reputation and are representative of the different design practices in The Netherlands. The main difference between these two companies lies in their organizational structure and work practice. While Indes represents the "small company" culture, Philips has a more complex organization structure, or a "big company" culture, that is expected to work differently from Indes. In brief, Indes focuses on working in small yet dynamic teams of all-round designers who are familiar with the whole process of design. On the other hand, Philips focuses on assigning responsibilities to specific roles in a development team, which specialize in delivering their own contribution.

By looking into the differences, this research aims to get a realistic perspective on the values that the support tool can bring to the design practice which comprises a variety of contexts and work cultures. From conducting the evaluation with Philips, in particular, recommendations can be proposed on how our approach using the tool can be adapted to the "big company" culture. As an additional aim, a multiday evaluation is conducted with Indes to probe for more practical feedback on setting up a support tool in real practice, which will be included as recommendations. This chapter describes the evaluation approaches with both companies and the findings.

\subsection{Evaluation with Indes ("small company" culture)}

With a continuous involvement of Indes designers from the requirement (Chapter 4) until conceptual phase (Chapter 5) and lastly the intermediate evaluation (Chapter 7), it is only natural to invite the same people again for the final evaluation. The evaluation is conducted in two parts: a focus group meeting and a multiday trial. With the focus group meeting, our aim is to find answers about the functionality of the support tool from research perspective. On the other hand, the multiday trial aims to discover the practical aspects for real implementation of the support tool.

\subsubsection{Focus Group Meeting}

The focus group meeting adopts the same setup as the one during the intermediate evaluation (Chapter 7). Similar purposes are being addressed, i.e. (1) to verifying the correctness of the implemented feedback/additional functionality, (2) to probe for more feedback to improve the functionality, (3) to evaluate the interface and interaction with the tool, and as an internal purpose, (4) to compare the overall quality of the final prototype with the intermediate one by means of a questionnaire. The meeting consists of three parts: (1) a presentation and demonstration of the tool functionality with examples using fictional data, followed by (2) open discussions, and concluded with (3) questionnaires. 
The eight participants have a similar composition as in the earlier evaluation, e.g. designer, usability engineer, electronic engineer, test engineer, and project manager. The participants reflect on how the tool would affect their practice. Subsequently, the discussion is mainly used to facilitate the participants pinpointing the additional functions that will make the tool really suited to their organization/practice. Their feedback and the questionnaire results are summarized in the later section 'Evaluation Results'.

After the focus group meeting is concluded, the next immediate step is to conduct a multiday trial in a real project setting. A project case at Indes is chosen to facilitate the trial. The next subsection describes the multiday trial in more detailed.

\subsubsection{Multiday Trial}

By allowing the designers to get an experience on using the tool a real project, this multiday trial aims to get a more in-depth feedback for the real implementation in practice. As the main functionality has been verified during several evaluation phases, the feedback from the multiday trial are expected to be on a very practical level. While this feedback will not be implemented any further, it will be included as recommendations.

During the focus group meeting, the designers did not have the opportunity to interact with the tool first hand. With a prolonged trial period, the intention is to get constructive feedback to improve the ease of use of the tool. For this trial, Indes has chosen to use the tool prototype for a real small-scale project that is still in the early definition stage. Two project members, a usability engineer and a designer both of whom were present at the focus group meeting, have agreed to use the prototype alongside their normal practice.

During the multiday trial, the two testers are asked to leave feedback either on the paper feedback form or the online one. The online feedback form is available on the prototype itself to easily accommodate feedback or the mention of bugs on a specific part. The multiday trial is planned for three to four days at the testers' convenience.

The multiday trial is concluded with a brief meeting with both testers and the project manager. Their feedback is quite concrete as in specific modifications that will allow their company to use the tool in the near future. Such enthusiasm from Indes strongly indicates that the support tool answers their needs and fits into their practice. The feedback from the multiday trial is summarized in the later section 'Findings'.

\subsection{Evaluation with Philips ("big company" culture)}

By approaching Philips, this research aims to find relevance of the support tool in the context of "big company" culture. While involvement of different departments and management levels in the company is preferred, this intention could not be realized due to limited resource. Accordingly the evaluation with Philips is done in collaboration with Application Research Center (ARC) department. ARC specializes in conducting user research and in representing the users (being their "voice") in a product development team. Although the findings are mainly from the perspective of ARC, an understanding of the organization structure, as will be explained in this section, helps the researcher to understand the implications of the findings to the organization as a whole.

The focus group meeting with ARC of Philips adopts the same setup as the one conducted at Indes, i.e. consisting of three parts: (1) a presentation and demonstration of the tool functionality with examples using fictional data, followed by (2) open discussions, and concluded with (3) questionnaires. Seven participants are present, 
mainly from ARC and one person from the development/engineering department. To guide the reader, this section will first introduce the organizational context and the way of working in Philips.

Within a development team that consists of a.o. marketers, designers, developers/engineers, the specialization of an Application Researcher is to translate user needs into functional requirements. In the specialized context of Philips, "designer" is the function that makes the outside appearance of the product while a "developer" develops the product as a whole system. At the start of a project, the marketing department provides a design brief containing commercial requirements which capture the consumer needs. Based on the commercial requirements, the ARC conducts further research on how the product will/should be used. When possible, the ARC would also research competitor products to see how they are being used. From the research, general use scenarios can be drawn, which will be the basis for functional requirements. These scenarios represent the main uses throughout the product life cycle. Using these scenarios as guideline, ARC delivers functional requirements. The development/engineering department elaborates these functional requirements into system requirements, which will be used as the basis for solutions.

To integrate the different levels of requirements, Philips uses Telelogic DOORSTM, an application developed by IBM for a specific purpose of requirements engineering. To be able to use the tool, individual investment is required to learn and adapt to its approach. As Telelogic DOORS is the official tool within the organization, the Application Researchers are dedicated to consistently use the tool in their projects. Furthermore, it is the formal platform to deliver requirements to other departments. While it provides a solid approach for requirements engineering, Telelogic DOORS has some limitations as summarized below:

- It does not integrate scenarios in its system. Every requirement usually has one or more scenarios as its rationale. Within Philips' practice, scenarios (including personas) are available on a different platform, requiring switching between both systems and sometimes also reduplication of information from one system to another. While Telelogic DOORS allows a requirement to be occasionally explained using scenarios, it does not actually accommodate scenarios as a recognized type of information. As a result, these scenarios are often not visible on the Telelogic DOORS platform. While this has not raised a big problem, a possibility to relate scenarios to requirements will definitely improve the traceability and reliability of each requirement.

- It assumes expert users and consequently, has a formal approach. Within Philips, an Application Researcher has developed a specialization in creating and organizing requirements using this formal approach. Individual investment is necessary to learn to use Telelogic DOORS. The use of Telelogic DOORS assumes that the design direction is finalized and that whatever design information in its system is more or less settled. The development team needs to be sure of and committed in the design direction. On the other hand, there exists a fuzzy beginning of a design process, where the design direction is not yet fixed and the team might not know yet which information is relevant. In such phase where creative thinking and brainstorming using tentative design information are necessary, Telelogic DOORS is not useful.

- It focuses mainly on textual descriptions. While requirements are indeed best formulated in solid sentences, their inspirations often come from flashes of images about use situations. While it is possible to include visuals on Telelogic DOORS, its main use is limited to display specific parts of the product as physical requirements (e.g. handgrip, shape, etc) or pictures of the competitor products in the design 
brief. The use of visuals for demonstrating information about users and use situations is not supported.

With a new context and a different type of participants, the focus group meeting with Philips has revealed different types of feedback for the support tool. While earlier discussions with Indes have focused more on how the support tool could fit into their practice, the discussion with Philips is mainly explorative on where and how the tool could fit overall in the organization. With specialized tasks and responsibilities, plus a currently running system Telelogic DOORS, the support tool is perceived as potentially mixing up the tasks of the marketing and ARC department. The division of responsibilities between identifying target users and getting insights in the identified users to the marketing department and the ARC respectively was initially not clear to the researcher. Consequently, the discussion started with making clear the specific parts of the design process in which ARC is involved. Some potentials of the tool have been recognized in ARC's relation with other departments. The participants pointed out how the tool could have been useful in a different way of working or setting in their organization. However, with the current practice and organizational structure at Philips, there is not much room for the support tool to offer much added value to the Application Researchers. The key findings from Philips are presented in the second part of the next section.

\subsection{Findings}

The following two subsections discuss the findings from the evaluations in the "small company" and "big company" culture respectively.

\subsubsection{Indes: "small company" culture}

The two methods of evaluation with Indes have reconfirmed the added values of the support tool in small collaborative design teams, of which their practice is representative. During the focus group meeting, the participants, even the ones whose functions are not directly related to users, can imagine using the tool in their day-to-day practice. This enthusiastic acceptance towards the tool is largely influenced by the company culture and the management. As Indes champions scenarios as a part of its user-centred design methodology, the management sets a mild policy that everyone in the project no matter his or her function needs to know who the users are. As a result, everyone is motivated to find out about the users and to not lose touch with them. The questions during the discussion express the feeling of engagement with the tool, e.g. "Can we use the tool for several projects?", "Can I reuse the information from one project to another project?", "Can I add my own category for scenario use phase?", "Can I link videos or photos to the actor profile?", and so on. Many of these questions lead to Indes-specific requirements, and therefore are considered outside the scope of this research. In brief, the participants are able to foresee the benefits of using the support tool in their practice. They identify the following main benefits:

- The informality and simplicity of the tool enables them to use it right away alongside their current tools. If it were too complex, they might need more reorganization to include the tool in their practice. In this case, everyone agrees that less is more.

- The online platform of the tool makes the user-centred information more accessible to any function within a design team. Currently the information is available in a formal document - often printed, which is basically a lean summary of all information the design team needs to know. The more informal representation using the tool, plus the accessible online platform, would encourage the team members to keep themselves informed. 
- The tool allows an efficient dissemination of Indes' collective knowledge on scenario-based design. By improving its user-friendliness and by incorporating a good introduction about using scenarios in Indes' practice, everyone with access to the tool can self-learn about scenario-based design on the go.

These recognized benefits have been reconfirmed with the feedback from the multiday trial. Furthermore, the multiday trial has also stimulated the testers to imagine how they would use the tool alongside their real practice. Consequently, they could come up with well-thought-of elaborate feedback to fit the support tool to their practice. However, similar with the feedback from the focus group meeting, most of the feedback from the multiday trial is either specific to Indes, too focused on the interface/interaction, or repetition of earlier feedback which has been agreed to be outside the scope of this research. Some comments however, are general enough and important to consider as a frame of reference for future development. The feedback from the multiday trial can be summarized as the following:

- Less flexibility and more restriction is preferred in documenting data using the tool. The prototype provides only general guideline, and does not prescribe the details on which data should be filled in. Initially intended to give more flexibility to the differing design practices, the testers expressed that such freedom could be interpreted as a lack of guidance especially with new or inexperienced members at Indes.

- A step-by-step tutorial/wizard can be added as a guideline on how to build scenarios and how to use them afterwards. While the activities related to building and using scenarios are mainly organization-specific, there is a general process on how it can be done. The prototype allows different entries to document scenario elements and to build scenarios from them. However, in a collaborative setting the designers prefer a more unified sequence to conduct this. The sequence can be made explicit to inform the designers the convention of their organization.

- The tool needs to give feedback to ensure the reliability of the information worked on by the design team. For instance, the design team often ask questions such as "Are we complete in the identification of the target users?", "Are these all the possible settings for the product use?", "Did we miss important scenarios?" and so on. While a good visual overview could help the design team answer these questions themselves, an extra security in the form of feedback from the tool will be appreciated. Such feedback can be implemented in e.g. reminder message in the interface of the support tool, summary of to-do tasks by email, etc.

- The relations of information need to be simplified to reflect a more pragmatic scenario-based approach. For instance, while goals of using the product theoretically belong to actors (users), in practice it is easier to identify goals as the designers think of scenarios. Similarly with the settings of product use, Indes prefers to associate settings with scenario use phases rather than with actors/products as implemented in the prototype. Furthermore, to maintain the simplicity of the tool, it should focus on aiding the creation and maintenance of scenarios and not on aiming to address all types of requirements. Remodelling the scope and the relations of information will need to adjust to the practice of the specific organization. Nevertheless, the preferences of Indes already inform this research of some simplifications that can be applied.

An important finding from the multiday trial is an affirmation of the mentioned benefits. Using the tool would not add extra effort to a design project at Indes, as their current practice is already in agreement with the approach using the tool. The support tool, when fully implemented, will make the dissemination of all the necessary design 
information easier. The online platform and the possibility to present the information in a more visual manner are certain added values to the design team.

Summarizing, the tool functionality has been verified with Indes as an answer to their needs. Additional functions and preferences that make the tool more fitted in their practice have been discovered and recorded. The benefits have been recognized by the different people with varied functions at Indes. The feedback from both evaluation sessions indicates that the support tool will integrate well with the practice at Indes.

\subsubsection{Philips: "big company" culture}

The focus group meeting with Application Research Center (ARC) at Philips has delivered valuable insights mainly through the discussion with the participants. With an organization structure that is different from Indes, a clear and strict work flow in the project, and participants who have a more specific role compared to Indes designers, the problem areas as described in Chapter 4 are less apparent in Philips. Furthermore, the availability of Telelogic DOORS provides a structured workflow among different departments. As explained in the previous section, Telelogic DOORS has limitations to support the application of scenarios despite its solid approach for requirements engineering. Nevertheless, it is satisfactory in its role to bind the works/results of different departments in Philips.

The feedback from ARC therefore comes from a different perspective, mainly on where and how the support tool could fit overall in the organization. This feedback suggests the ways to improve the support tool to other specific needs in industry at large. This section summarizes the collective opinions of the participants during the discussion; collective either because the opinion is mentioned by several people or because it is from one person and agreed upon immediately by the others.

As the first step, the participants have identified the types of projects in which the approach using the support tool can be potentially useful. In the case of big organization like Philips, a project could benefit most from the support tool when it is still in the early stage, at the fuzzy front-end of a design process where the direction, target user, or need in the market is unclear. Furthermore, a design team that integrates different disciplines and functions would also be helped by having one system that is accessible to all. On the other hand, a project for incremental development, successor products, or small-scale redesign is not considered a suitable application area for the tool. This opinion is understandable from the point of view that a follow-up project already has the most part solved and documented using another system; using the support tool in such a project would mean extra work moving data from the old system. A complex organization structure may also hinder the adoption of the tool due to its stance on being informal. There exist internal and external formal relationships in big organizations like Philips, which demand strict communication lines between the involved parties. The information needs to be finalized first before being passed to another department/function, for instance. With these constraints from within the organization practice, some key issues and suggestions are classified in the sections below.

\section{Responsibility and scope of using the support tool}

To be useful in the later phases, the tool needs raw design data to be documented on its system. This raises a question on who should be responsible for filling in the data. Only specific parts of the tool are directly related to the tasks of Application Researchers. For example, while the functionality to document actor profiles (personas) and scenarios can be useful, the requirements part is already handled by the currently used tool (Telelogic DOORS). Documenting user data is not entirely the responsibility of ARC. One 
possibility as suggested by the participants is to fill in the user data together with the marketing department - which is located in a different place. Such new proposed practice is therefore not immediately applicable. It would require a shift in the organization culture. The management needs to approve that such a shift does not disrupt the design process as a whole.

\section{Interfacing with other tools in the organization}

An organization creates its own unique practice by defining an approach. The more complex the organization, more tools might be needed to support the specialized departments/functions involved within the organization approach. In the case of Philips, different departments have their own autonomy to perform their responsibilities. The integration of the results therefore needs a special attention. The situation is not always ideal as for example, the Application Researchers need to retype data from the marketing department into the tool they are using (Telelogic DOORS). The ARC strongly expresses that a new support tool in the organization has to change their practice as little as possible; in other words it needs to have good interfaces with the current formats of deliveries. For instance, a possibility to export and import data is a must. With the current specific formats for input and output, the support tool needs to support the process between the two types of deliveries and reduce menial works as much as possible. In other words, it should fill in the "gaps" between activities by providing precise interfacing.

\section{More visuals as inspiration}

While visualizing the information is recognized as an essential functionality for the tool, it is also organization-specific. As mentioned earlier in Chapter 7, visualization is not within the scope of this research and is not addressed in the prototype development. Nevertheless, the feedback in this category is addressed here as it is a part of the findings at Philips.

In the case of Philips ARC, the formal tool Telelogic DOORS relies heavily on text, therefore it is necessary for a complementary informal support tool to focus more on visuals as inspiration. Scenario creation needs to be a creative visual exercise with playful interactions. Especially for the ARC department at Philips, whose main focus is to deliver bullet-proof product requirements, it needs to be an inspirational process. Some suggestions have been made regarding the kind of visualization most useful in the practice of ARC. Briefly, the suggestions cover the following:

- Less text and more pictures in the tool, especially in actor profiles and scenarios, with a possibility to create printable cards for actors

- Scenarios can be represented using flow charts to improve readability

- Overview diagrams to show where particular actors, settings or events are used in scenarios

- Database of images showing inspirational use situations, e.g. various pictures of misuse can be randomly displayed to inspire an Application Researcher of critical scenarios that lead to crucial requirements

Summarizing, the suggestions from Philips ARC have been largely covered in the earlier sessions with designers from Indes. Both agree that in real implementation, the tool will need to focus on visualizing the already covered design information, and not on making it more extensive. The support tool can remain simple as long as there is a good interfacing with other tools. The feedback therefore serves as a confirmation towards the direction to which future development should go. 
The findings, supported with the questionnaire results (as presented in the next section), suggest that the support tool is less applicable in a "big company" culture. A big organization tends to have a more rigid approach, and well-structured job functions that perform specific responsibilities and interact with one another through formal deliveries. In the case of Philips, the tool functionality does not encompass aiding the tasks of one particular function, and is rather spread among different functions. Any departments dealing with user data might find the support tool useful to work with internally. However, the result/delivery from one department will need to be transferred to other departments in a predefined format. The integration of results is already served by another tool Telelogic DOORS. Although this integration functionality is also a potential benefit of the support tool, the already applied tools in Philips would make this offered functionality redundant. Consequently, in this situation the adoption of the support tool might add value on departmental level, but is not considered to add much value to the communication and cooperation processes on the organizational level. On the other hand, a "small company" culture tends to have a more open approach in the sense that the different functions in a design team interact more informally. The support tool fits this latter context better and offers a collaboration platform for the design team. The support tool, especially with its accessible online platform, allows an easy dissemination of information in the design team, and subsequently creating a unified guidance on SBD practice in the company.

From another perspective, the support tool can be perceived as more valuable when the design direction is not yet mature. Depending on the work culture, design companies work with design briefs with varying levels of detail. The evaluation session with Philips ARC revealed that in their approach the design direction is more or less set at the beginning of a project. While at Indes, it is more often the case that there is still room to reshape or redirect the design direction. The nature of the tool is more accommodating towards the latter approach.

To conclude, the adoption of the support tool in a big organization like Philips would need a commitment to reshape the current way of working. Such commitment can be encouraged by providing good interfacing in the support tool, which guarantees seamless integration with other tools. Furthermore, endorsement from the decision makers in the organization might be necessary to stimulate the change in the first place. 


\subsection{Questionnaires}

Similar with the intermediate evaluation (Chapter 7), the questionnaires in the final evaluation aims to get an indication of the overall quality of the prototype. The questionnaire setup in the intermediate evaluation is reused in this final evaluation. The minor changes include the reformulation of some questions, without changing meaning, to improve their clarity to the participants. By using the same setup consistently, it is possible to compare the different results. For instance, this comparison between the scores from the intermediate and final evaluation with Indes could reveal whether the final prototype is an improvement from the previous one. Figure shows the comparison of the average scores by Indes designers during the intermediate and final evaluation.

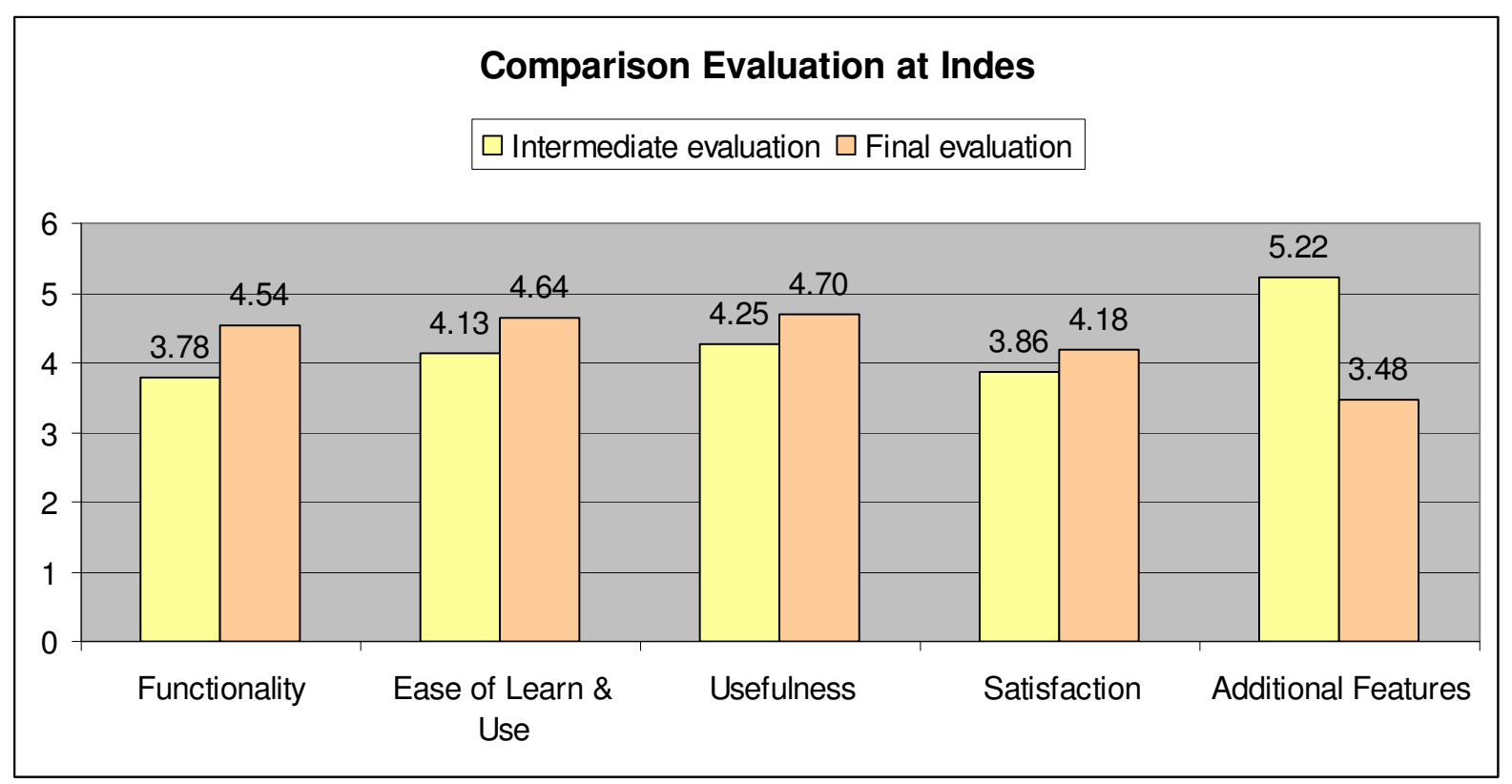

Figure 9.1: Comparison of Indes' average scoring during intermediate and final evaluation.

The final prototype receives higher scores overall in each category, except in 'Additional Features'. This indicates that the additional functionality as agreed in the intermediate evaluation as well as the feedback from Indes designers, have been implemented correctly, although still yet not completely. Furthermore, the reason for a lower average score in the category additional features is because Indes designers strongly suggest keeping the tool simple, to avoid the tool becoming too complex to fit into their lean design practice. In accordance with Indes' practice and work culture, they prefer the lean approach of using the tool to ensure that they can remain flexible and dynamic as a team.

A comparison between the average scores of Indes and Philips ARC (Figure 9.2) clearly indicates a lower acceptance from Philips. As explained in the previous section, ARC does not consider the support tool to be easily fitted into their current practice, thereby limiting the added value of the tool to their department. While other departments dealing with user data might find the support tool useful to work with internally, the integration of results from each department requires a formal approach. This is currently already addressed by another tool, i.e. Telelogic DOORS. Appendix 6 presents a more detailed analysis on the six categories being evaluated. 


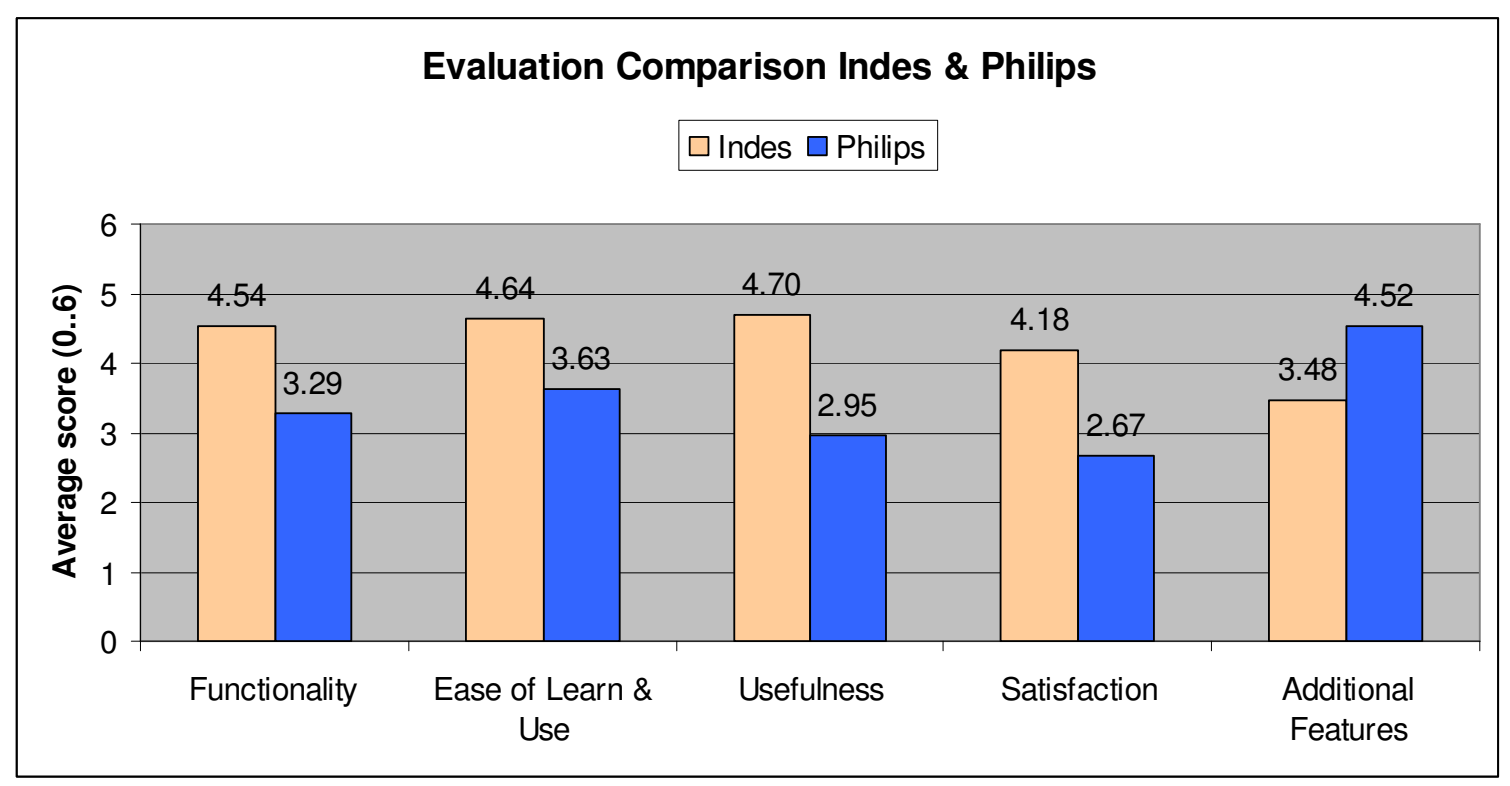

Figure 9.2: Comparison of final evaluation scores between Indes and Philips.

The questionnaire results show that the final prototype is an improvement from the intermediate one. This means that by taking into account the feedback from the intermediate evaluation (Chapter 7), the prototype development has proceeded in the right direction. It has also confirmed the difference between different organizational contexts (represented by Indes and Philips) in assessing the fittingness of the support tool. Lastly, the questionnaire results also indicate the aspects of the tool that need further attention. Within this research project, the prototype will not be developed any further. Nevertheless, the potential improvements, including the qualitative feedback, will be included as recommendations for future work.

\subsection{Conclusion}

This chapter has described the evaluation with representatives of two different design practices, i.e. one small and one big company. The final prototype, as the essential mean in the evaluation, has successfully captured our idea for an approach that guides the initial documentation of design information, creating scenarios to make sense of the information, and sustaining scenario uses in the design process. Both practices recognize the potentials of the support tool when it remains informal, simple and inspirational. The activity of creating scenarios is a creative process which would be supported by making the design information more visual. While visualizing information is outside the scope of this research, this topic is acknowledged as a necessary functionality for the practical application of the tool. Additionally, the evaluation with both companies, including the multiday trial, has revealed other topics of attention concerning the practical use of the tool.

The application of the support tool in real setting generally needs specific adjustments from both sides: fitting the tool functionality better to the particular practice and reorganizing the practice to make the best use of the tool. This means the adjustments will depend on what is shaping the design practice, e.g. the people, the organization structure, and the nature of the project; therefore it is not possible to propose a generic formula. The findings from the evaluation within the "small company" culture have discovered potential areas of adjustment, which will need to be defined together with the particular design practice. These areas mainly concern the right amount and the form of guidance to the users/designers in adopting the approach proposed in the 
support tool, and the custom-fitting of the scope and relations of information in the support tool with the work practice. Within the context of a "big company", further concerns involve the definition of scope and responsibilities in using the tool, and the interfacing with other formal tool(s) being used next to it. The integration of design artefacts across different roles and functions is a priority in this context. Although this integration functionality is also a potential benefit of the support tool, it is quite often already implemented in the company by means of other tools (as in the case of Philips). Consequently, in this situation the adoption of the support tool might add value on departmental level, but is not considered to add much value to the communication and cooperation processes on the organizational level. The implementation of the support tool in a "big company" context would therefore require recognition of a large added value on the departmental level. Implementation would furthermore comprise: (1) more extensive tool functionality to cooperate with other tools and address the more formal work practice, and possibly also (2) changes in the practice or structure of the organization. Overall, this would mean a deeper commitment from the organization.

The findings from this final evaluation have clarified the potential added value the support tool can offer for the design industry. Recommendations for future work have been proposed to realize this potential added value. The lessons learnt from this research and the recommendations will be concluded in the next and final chapter. 

Part IV

Conclusions and Recommendations 



\section{Conclusions and Recommendations}

The research aim was to provide concrete support for creating, using and managing scenarios for the particular domain of product design.

With the trend of technology developments and their ready applications in consumer products, nowadays products are generally growing more complex in functionality. They also tend to have a greater degree of dynamic use, due to being more portable, multi-functional and accessible by a wider range of users. In designing such products and ensuring their success in the market, usability is a key priority. Usability is shaped by the quality of user-product interaction. Therefore the users need to be taken into account in the design process. A design approach, generally referred to as UserCentred Design (UCD), brings the focus closer to users. UCD essentially collects and uses information about users (e.g. their characteristics and goals) and use situations to incorporate usability early on in the design process. An effective and efficient communication with users and other stakeholders is necessary in this approach. Especially within complex design projects that often demand multidisciplinary design teams, numerous stakeholders are involved. Scenarios are expected to be a useful means to support the communication in the design process. As concrete narratives, scenarios facilitate making explicit the future use of the product being designed. The discipline of Scenario-Based Design (SBD) guides the use of scenarios to support the design process. SBD was first applied in the development of early interactive systems. Later supporting work is often tailored to specific domains or cases, making the developed SBD approaches not directly applicable to the product design domain, which is addressed in this research. Further research is needed to complement and customize SBD for application in the product design domain. This research in particular aimed to provide the support to guide designers in effective and efficient use of design information within the product design process through the application of SBD principles.

This thesis has argued why using scenarios could help the process by allowing the involvement of users/stakeholders in the process, accommodating quick and early evaluation of the product, and therefore highlighting usability issues early on (Chapter 2). However, alongside these promising benefits, there are challenges in integrating scenario use in a design process in practice. Starting off with the general challenges related to the lack of concrete guidance for creating, using and managing scenarios, specific design activities that potentially benefit from scenario use have been identified in product design practice (Chapter 4).

Based on the knowledge of SBD, an approach for scenario creation, use and management in (consumer) product design has been developed, to contribute towards a more solid Scenario-Based Product Design (SBPD) discipline. To ensure relevance for the design practice, collaboration with design practitioners has been pursued throughout the various phases in this research. Combining these two worlds, this research contributes in a practice-based support tool that deals with effective and efficient use of design information by applying SBPD theories and principles.

\subsection{Conclusions}

To the design practice, many design approaches, methods and tools are available, yet many of them are not used. Most practitioners are critical when it comes to adopting a new support in their organization. To be acceptable, a design tool needs to explicitly add value and enhance their work practice instead of burdening them with extra tasks. 
Therefore, to build a fitting support tool, this research has addressed dedicated research questions that lead to the practice-based requirements. This section presents our conclusions as answers to the research questions as formulated in Section 1.1.

\section{RQ1. What makes the use of scenarios relevant in consumer product design?}

SBD aims to make use explicit by means of concrete narratives so that a design team can discuss and analyze how the technology or product fits into people's activities (Carroll, 2000b). This allows usability studies to be an integrated part of the design process, not as detached post-design testing (Carroll, 1995). Consequently the process of using the new system is the end-result of design, as opposed to the system alone (Kuutti, 1995). This motivation resonates with user-centred design in general, but is especially amplified in the context of consumer product design. Firstly, with the technological advances, nowadays consumer products tend to be complex in their functionality. Secondly, the dynamic use situations of the products require the design team to keep track of different aspects of design information. Designing such products therefore implies being confronted with a large amount of design information and not uncommonly contradicting requirements to be dealt with. This situation demands an approach that makes the design rationales easily accessible. This way the design team can propose reasonable trade-offs to accommodate these contradicting requirements while at the same time maintain a good usability of the product. Scenarios are a lowcost, easy and accessible tool/medium to capture the rationales, elaborate potential solutions, and discover where usability problems might arise. The application of scenarios in the specific context of consumer product design, as guided by the SBPD discipline, is therefore relevant.

\section{RQ2. Why is further research in the SBPD discipline necessary?}

This research question can be answered from different perspectives. Firstly, the existing SBD/SBPD approaches might not have addressed all relevant aspects of design activities. As Dorst (2008) points out, design methods and tools developed within the design research community often ignore the content (the design problem and the emerging design solution), the actor (the designer or the design team), and the context in which the design activities take place. They focus mainly on enhancing the design process as if it were isolated from the other aspects. Chapter 3 has described a survey on support tools for scenario construction and management which have been developed within the design research community. The survey confirms that these available approaches are not in complete touch with practice. To avoid this pitfall, this research aims to deliver a SBPD approach with practical values that takes into account the content, actor, context, and process of a design project.

Secondly, this research adapted knowledge from the SBD discipline for application in consumer product design. The core knowledge of SBD was developed and applied mainly in the development of early interactive systems (i.e. computer applications), which have different characteristics from (tangible) consumer products. Early software applications concern a more limited set of interactions and use situations. Furthermore, they are often designed for a specific type of users, with known characteristics, skills and level of experience. Interactive systems have since evolved into becoming more mobile, rich, personalized, and generally more complex. These characteristics are also present in tangible consumer products, which integrate more and more software/system functionality. In brief, nowadays consumer products have a larger degree of dynamic use - due to being more portable, multi-functional and intended 
for a wider variety of users, which was not the focus of attention in the early SBD framework. Owing to these dynamics, the translation of SBD to the product design domain (SBPD) requires additional knowledge.

Summarizing, further research in SBPD is needed to support the domain of consumer product design and its design practice. As a stepping stone, Chapter 3 has presented a scenario use roadmap which has been developed to be a discussion tool with practitioners in pinpointing potential areas of improvement in current scenario use. Through this collaboration, the direction for further research has been refined to answer specific design activities (RQ3).

RQ3. What activities in product design practice can be supported by the use of scenarios?

Through collaboration with design practitioners, design activities for which the practitioners expressed the need for support have been identified. These activities are in accordance with the initial ideas to support the creation, use and management of scenarios. The identified activities are (1) the initial documentation of design information, (2) creating scenarios to make sense of the information, and (3) sustaining scenario uses as an integrated part of the design process. Chapter 4 has elaborated these support areas; for clarity they are summarized here:

1. amidst the "sea" of design information, scenario building gives a sense of direction to identify and document information pieces that are necessary (i.e. scenario elements),

2. designers could be inspired to create meaningful scenarios by reusing (combining) the scenario elements,

3. integrated scenario use in the design process needs a good management, to make it easy for designers to find scenarios for specific purposes.

RQ4. How can another form of support be useful for the identified activities (RQ3) in a product design process?

The design research community has contributed approaches, methods and tools for supporting scenario creation and management. However, as discussed under RQ1, they often do not accommodate the actual needs of the design practice. There is room for another form of support for SBD in design practice. As stated under RQ3, the collaboration with the design practice has revealed a willingness from the practitioners to explore practical support in the particular activities related to documenting and processing design information. Amidst the available tools that appeared to be less useful in practice, the support needs to take on a specific form that fits the design practice, while also providing the much needed structure and concrete guidance for scenario creation, use and management. For the practitioners to accept and adopt such support, it has to meet certain characteristics:

- Flexible and independent, its adoption should not be dependable on other methods and tools in the design process, and vice versa.

In accordance with this characteristic, the form of a design tool is considered to be most appropriate to allow more flexibility and independence as opposed to for example a methodology. The role of such tool would be to combine and process the results of other used methods and tools. Evaluation with practitioners (as described in 
Chapter 5, 7 and 9) has in addition emphasized three other distinct characteristics of the tool:

- Informal and creative, fitting in with the nature of creating and using scenarios which is to "sketch" the use and inspire functionality of a product,

- Simple and lean, avoiding the tool being too prescriptive and exhaustive,

- Providing a framework for scenario use, showing the possible ways to use scenarios throughout a design process.

Summarizing, the tool will be most acceptable when it remains informal and encourages creativity. The effort to create and maintain scenarios is considered justified as long as the scenario use is sustainable, and there is good interfacing with other activities in the design process.

RQ5. In which stages should the support be gradually actualized?

In Chapter 6, an overview of the evaluation plan has been presented, with the mention of media/tools and methods used in each evaluation phase. The basic functionality of the support tool has been proposed based on the characteristics identified by the design practice (as concluded in Chapter 4). Different levels of prototypes have been developed to demonstrate our ideas and to evaluate them with design practitioners. Chapter 5 has described the conceptual design that shows the behaviour and supposed use of the tool. This concept has been evaluated with design practitioners by means of mock-ups/drawings and scenarios. Later on interactive prototypes have been implemented as a web-based application (Chapter 7 and 8). The interactive prototypes allow further exploration and evaluation of the interface and interaction that provide the functionality. To represent the realistic target users of such support tool, the evaluation has been conducted with different types of design practitioners, i.e. with different levels of experience in using scenarios and different work contexts ("small company" and "big company" culture).

\section{RQ6. For which industrial context will the support tool be applicable?}

The prototypes have been verified with various practitioners representing different design practices. The support tool, as demonstrated using the prototypes, is coherent enough with the design process in general, as well as easy enough to be understood by novice in SBPD. The involved practitioners have recognized the potential of the support tool to disclose use-related information, making design rationales from the perspective of the users known to different roles and functions in the design team. Chapter 9 discusses the evaluation of the tool that included the comparison between "small company" and "big company" cultures. The findings from the comparative evaluation indicate that the support tool is more suitable and can be integrated more easily in the context of "small company" culture. The tools suits a more informal way of working, often involving all-round designers working in close-knit teams, which is generally found within "small company" culture. The work practice in a "big company" on the other hand, tends to be more formal as the many functions or departments need a structured approach to ensure a solid integration of results. Although this support tool offers functionality for result integration, within the latter context it is often the case that other tools are already implemented to address the integration processes on the organizational level. Consequently, the added value of support tool on the organizational level might be limited by what is already implemented. However, the work processes of specific roles or departments in the organization might still benefit 
from the tool functionality. Such recognized added value on the departmental level could well justify the adoption of the support tool in the "big company" context.

To conclude, this research contributes in a practice-based approach to create, use and manage scenarios in a product design process. The criteria for a tool that supports this approach have been identified and verified. The prototype of the support tool, as evaluated with the practitioners, has offered the aimed for guidance for a scenariobased design process. This research has revealed that the availability of support increases the willingness of design practitioners to use scenarios in their projects. The support tool, as proposed in this research, has been assessed as being useful for delivering its purpose, specifically in the context of "small company" culture.

\subsection{Reflection}

With the research questions answered, this section will reflect upon the approach that has been followed.

\subsubsection{Has this research followed a framework of rigor and relevance?}

The researcher has performed comprehensive studies on SBD in different application areas, as well as on methods, tools, and approaches that compose the body of knowledge of the discipline. An analysis has been conducted to formulate a new direction of research in SBPD, i.e. to support the designers in creating scenarios and organizing their use, as this seems largely unguided in the product design practice. Our complementary studies on existing tools that address this purpose have revealed a lack of applicability in real design settings. This finding confirms the trend argued by Dorst (2008), in that available methods and tools developed within the design research community are often too focused on creating a more efficient and effective process, while missing on other aspects found in the design practice (i.e. actor, content, context). This theoretical match has motivated us to find relevance between this research and the design industry.

There is a clear industrial relevance in this research with the involvement of design practitioners in different phases. Although a large scale involvement has not been feasible in this research, the involved representatives of various design practices have given a sufficient coverage of the target users and their contexts. The direction of this research was informed by the knowledge from design practice, and the proposal for a support tool has been confirmed by our industrial partners.

\subsubsection{The Involvement of Industry}

This research has involved practitioners representing different design practices in the different phases of development and evaluation. Although the support tool has been developed with the practice of a small/medium-sized design agency as a reference, its applicability in a large-scale design company has been explored. The researcher is aware that the two representatives of "small company" and "big company" cultures respectively cannot be generalized as the design industry at large. Nevertheless, the findings from the studies conducted with these representatives have identified the potential applicability of the support tool in both contexts. To further verify this potential, future work could involve more varied representatives of the design industry, as suggested in Section 10.3 Recommendation.

\subsubsection{The Use of Scenarios}

The researcher has used scenarios extensively to probe and verify both problems and solutions. While the prototypes were being developed, our scenario-based approach has been able to communicate our ideas early. For instance, while probing the design 
practices, scenarios and mockups/drawings have allowed efficient and effective communication about problems and potential solutions at once. In the later stages, the scenarios were accompanied by a prototype to show how the support tool is intended to be used. The combined use of scenarios and prototypes has proved valuable in this research.

\subsubsection{Drupal as Development Platform}

With every development platform, there is always a trade-off between capabilities and limitations. The use of Drupal as development platform has involved a compromise between the achievable development pace and faithful translation of the interfaces and interactions as proposed in the conceptual design. A consistent implementation of the main tool functionality has however been ensured by the choice of Drupal (see Chapter 6). The prototypes have been developed with the aim to demonstrate the approach for documenting design information, creating scenarios and organizing their use using the support tool. Therefore the single limitation, i.e. the fact that the interaction and interface of the prototypes have been developed according to what was feasible by means of Drupal, has not affected the core quality of this research. It can be concluded that Drupal is highly functional for the prototype development in this research.

\subsection{Recommendations}

This research, and the development of prototypes that demonstrate the support tool, is concluded with the production of this thesis. In the bigger picture, SBPD as a discipline needs more concrete work for design practice to benefit more from it. This research has addressed specifically the practical activities of creating, using and managing scenarios. Scenario creation is a process that is shaped by the people, the organization and the nature of the project. Consequently, not one generic solution can be proposed. A continuation of this research can therefore be foreseen as an adaption of the knowledge and the support tool to be more applicable in industry.

This research has delivered answers to the research questions and a proposal for a support tool. Nevertheless, additional steps can be proposed to make the results more solid and the proposed support more acceptable by the industry. These recommendations largely concern a deeper involvement of design practitioners, as summarized in the following:

- More in-depth practice-based studies. The general rule in this research has been to understand SBD through literature studies, and then verify it with the design practice. For instance, our scenario use roadmap (Figure 3.6) has been formulated as a generic synthesis of possible scenario uses in design processes. The workshops with Indes have served to verify the roadmap, among other objectives (Chapter 4). This process can be complemented with additional focused studies on particular design practices, which should represent different work cultures (e.g. "big company" versus "small company"). Instead of only verifying the generic scenario use roadmap, the involved design practitioners could be facilitated to generatively build their own tailored scenario use roadmaps. This would provide this research with an even more valid representation of scenario uses in the design practices. Additionally, the practitioners could benefit from making explicit their own understanding of the current and potential scenario uses in their particular work practice. Such understanding could inspire them should they wish to reorganize and improve their practice.

- Collection of problem scenarios from diverse practitioners. Problem areas in design practice have been identified by means of workshops and explorative 
questionnaires. These identified problem areas were translated into problem scenarios by the researcher, and then verified again with the design practice. This research can benefit from engaging the design practitioners into contributing their own problem scenarios. These practitioners should represent different roles or functions in design projects, and preferably also from different company cultures. The resulting scenarios can give more insights in the target users as individuals and their work contexts, which will inform further development of the support tool. To ensure commitment from the practitioners, the researcher will need to provide a clear aim and guidance in the process.

- Survey of design tools applied in the design practice. In practice, the support tool has to work alongside other tools. Further research can be done on how the functionality of other applied tools would affect the intended use of the support tool. Accordingly, further development of the support tool could be adapted to ensure a better integration with other tools.

- All-round evaluation of the support tool. The support tool has been recognized as being more suitable for "small company" culture (RQ6). While the prototypes have sufficiently confirmed the acceptance of the tool and the corresponding approach, an all-round evaluation using a fully implemented support tool will further confirm the added value in practice.

For real application in industry at large, the support tool will require additional development. The findings from the evaluations (Chapter 5, 7 and 9) have indicated the additional functionality that would be needed for the actual use of the support tool, as summarized in the following:

- Interfacing and integration with other methods/tools: this functionality entails the possibility to export/import data as well as defining input/output to fit the tool with the way of working in the particular organization,

- Visual overview: this is a highly appreciated functionality since visuals assist the designers to easily grasp overviews, spot patterns, and identify other important information; some inspiration can be learnt from existing applications e.g. Liveplasma? (example in Figure 10.1),

- Custom-fitting the scope: while the support tool proposes a lean scenario-based approach, adjustments should be possible to include or leave out parts depending on the need of the organization or project, e.g. actor profiles might need to be more elaborate depending on the nature of the project,

- Definition of roles and access: the support tool needs to provide access management, which would depend on the roles and responsibilities within the particular organization. 


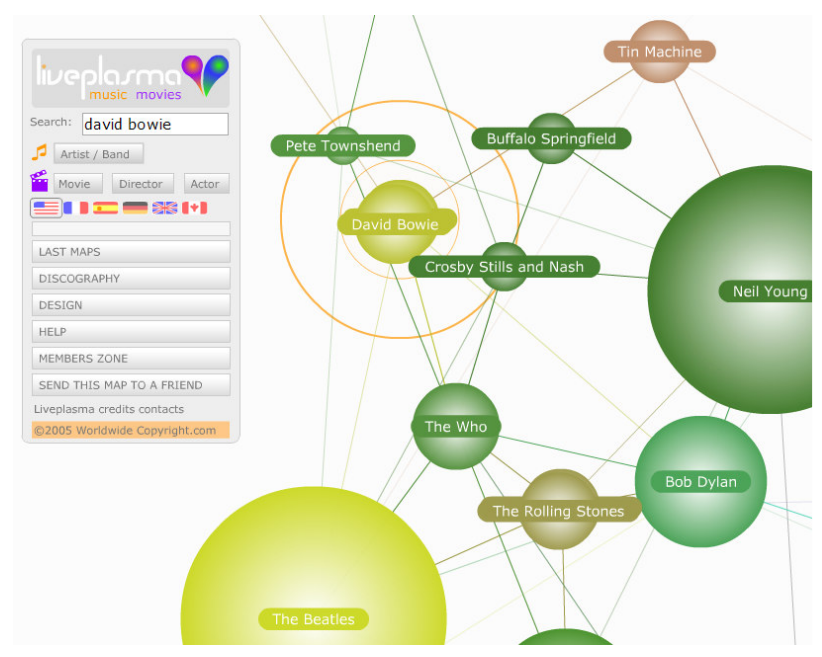

Liveplasma search on 'David Bowie'

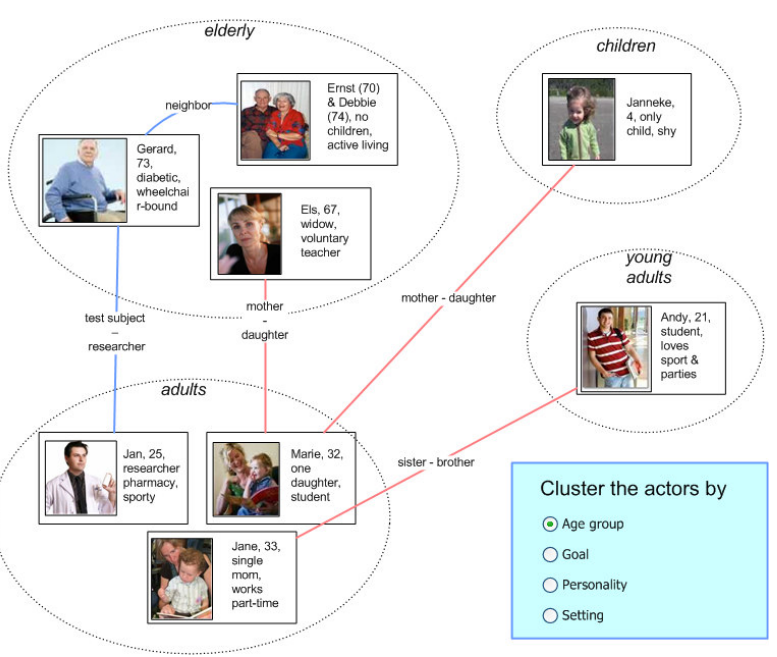

Overview of actors clustered by age group

Figure 10.1: Interpreting Liveplasma music graph into a visual overview of actors and their relationships.

\subsection{Future Directions}

Supporting scenario creation, use and management in SBPD could be extended with the adaption of research in other disciplines. For instance, it is not far-fetched to say that some Artificial Intelligence can be part of the support tool. For instance, to support the management of scenarios, an intelligent agent could check for completeness or coverage of scenarios and occasionally remind the design team: "Hey, you forgot an important scenario with actor $X$ when he is in situation $Y$." Other work in narrative generation (e.g. Swartjes, 2010) can be inspirational towards automatic generation of scenario storylines. However, the findings from collaboration with industry indicate that scenario generation requires imagination, intuition and life experiences of human, and is therefore too difficult to be replaced by Artificial Intelligence. The inclusion of $\mathrm{Al}$ in the support tool should therefore first focused on assisting scenario management, and remain only supportive towards the creative intelligence of designers.

The support tool can potentially grow in usefulness especially with its use over time across different projects as well as by a growing number of collaborators. Firstly, the tool allows the information documented in its system to be reusable in other projects. This information often comes from other sources like user and market researches; therefore over time the tool could be a reliable knowledge-base for a specific design domain. Secondly, the online platform of the tool allows a large-scale collaboration independent of location. To illustrate the tool's potential, two variables are recognized whose combination of instances may unfold different applications of the support tool. These variables are the company culture (big or small) and the target market (global or specific). Four scenarios have been developed to illustrate plausible applications of the support tool within the frame of these two variables (Appendix 7). Of these four, the scenarios involving "small company" culture dealing with both global and specific markets are considered to be most easily adopted. This consideration is largely motivated by the observation that the "small company" culture shows a nature of people's initiatives and experiments to adopt new ideas/approaches/tools and a culture that naturally stimulates it. Although a larger adjustment is necessary, the scenarios concerning a "big company" culture are also realistic. All in all, all four scenarios are likely to become reality not too far in the future. 
Appendices 



\section{Appendix 1 Explorative Workshops at Indes}

The following is an excerpt of the briefing letter sent to participants prior to the workshop. It gives the participants an idea of what they are going to do in the workshop and the fictive case study that is going to be used.

\section{The Case Study}

Speeda Inc. is a medium-sized company producing bicycle equipment and accessories. Their products are among others: bicycle computers, lighting sets, locks, brakes, bicycle bags and baskets, and bicycle bells. Speeda Inc.'s current market is steady as their products are at the top of developments. Their design department, however, is small and quite conventional. The designers at Speeda Inc. tend to follow the mainstream of product development and only make small improvements to the usual product designs. The main reason is that they want to play safe and keep hold of their current market.

Recently, a known bicycle producer Batavus held a student design competition with the assignment of designing a means to transport baggage on bicycles. The winner designs are very innovative and inspirational, but most of all, they are no conventional bicycle bags or baskets. Speeda Inc. is concerned about this, and decides to make a similar move and innovate their product lines of baggage transport means for bicycles (which so far only consist of bicycle bags and baskets). Speeda Inc. perceives that the near future market is still stabile because Batavus the competitor would need at least a few years to mature their new baggage transporters. For this reason, Speeda Inc. wants to target a market 5-10 years in the future. As mentioned before, the designers at Speeda Inc. are conventional and need to learn much about designing for the users. One of them has had an initiative to ask a few bikers, who happen to be his friends and family, about their experiences with carrying things on their bikes (their stories are summarized below). His fellow designers are not convinced this is the right way to start. So, they decide to assign this case to another more experienced design bureau. Speeda Inc.'s designers, however, still want to be involved lat least be informed about every step).

Your design team is hired by Speeda Inc. with the assignment "to design a novel means to carry baggage on bicycles that should make life easier". Speeda Inc. does not know for certain whom the target group is. Additionally, their definition of 'baggage' is very loose. "That's why we hire you" was their answer when you asked. Most likely Speeda Inc. will not object if you decide anything for this case as long as you have arguments for it. However, Speeda Inc. limits that your design solution is a kind of bike accessory which works with most types of bikes. Knowing that you have quite some freedom, this case could be a good opportunity to learn about scenario based design "on the fly". Baggage carriers for bikes have diverse users, purposes, use situations and possible solutions which your fellow designers and stakeholders might not be aware of.

\section{$\underline{\text { User Stories }}$}

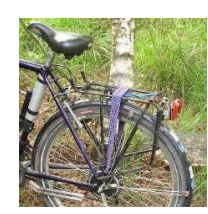

"I use the elastic cords on my baggage rack to fasten my gym bag [l already carry a laptop backpack]. I always feel a bit worried that the bag would be unbalanced and fall so I check now and then by glancing behind me...I wish my gym bag would just stay there firmly."

\section{Tjerk, 20 year old, a sporty college student}

"I used to have a pair of wire baskets with hooks attached to my

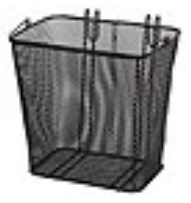
baggage rack for groceries...sturdy and practical. But, since I installed the child seat, there is no space to attach them. If I do spontaneous shopping [e.g. after picking up my son from daycare], I wish I always have a space to carry my groceries comfortably." Sarah, 34 year old, a practical working mom with a toddler

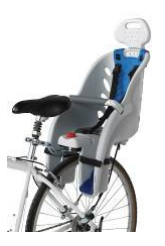




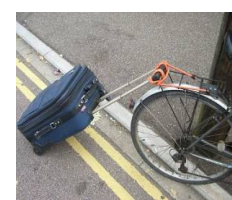

"I often travel by train for work and personal purposes... which usually takes a few days. I live quite nearby the central station, so here is how I do it. This would have been perfect if my luggage wheels are less noisy and there is a simpler way to attach it other than using a bungee cord." Alfred, 31 year old, an ecofriendly academic researcher

"I do most of my grocery and other shopping on Saturdays. Since I visit different sellers on the market, I get to carry a lot of things before I reach my bike [which I usually park across the market]. My bike shopping bag is not meant to be carried around, it seems... I want something trendy and easy to carry around, but not with rollers. Those are really old-fashioned!!" Betty, 23 year old, a friendly nurse who works long hours

\section{Trends}

There are some observable (read: fictive) changes in society and technology that you can take into consideration to your design. Please remember that your design is meant for a market 5-10 years in the future. Some of these trends could be amplified by then.

People are adopting 'green and healthy' lifestyle. These people have enough spending power to demand for products that are ecological, green, energy-saving, responsible and recyclable. Biking is still popular as a means for transportation and recreation. "If you can travel the distance with a bike, then use a bike" is the popular attitude among the people.

There are rumours that the government will introduce a 'green' system. With this system, the government would be able to trace any household in The Netherlands for their energy consumption (e.g. petrol, electricity, gas) and recycling activities (e.g. the amount of trash they recycle and other 'green' behaviours). Hopefully, the households with more 'green' attitude would be rewarded in each year end.

Technologies have developed a lot during recent years, especially the ones related with security. While iris, ear and facial biometrics are still exclusive for airports and national identity database, fingerprint and voice recognition have become more secure, affordable and accessible for daily products requiring authentication. For example, most house doors in middle to upper class neighbourhoods are nowadays equipped with these technologies to grant access to the right people.

These trends are some imagination seeds that you could use for developing certain types of scenarios. If you know of any more technologies that inspire you for a futuristic solution of this case study, please feel free to share them during the workshop.

A few little extra things to prepare:

- to get familiar with the case, you might want to gather more stories from your family, friends, or neighbours about their experiences with bicycle, baggage and the means to carry it (bags, panniers, trolley, etc). In this case, please organize your stories so that you can present them quickly (during 'Revisiting the case study \& preparative task' below).

- you are allowed to start researching this case or even prepare some (existing) design concepts before the workshop 


\section{Appendix 2 Explorative Questionnaire}

The complete questionnaire also includes an introduction letter to the participants, summarizing this research project and our motivation to conduct this questionnaire. For brevity, this appendix only lists the main content of the questionnaire.

\section{Challenge 1:}

Designers are trained by education and occupation in getting information relevant to the design project. This could be by researching existing products or competitors, technologies that may be useful, and most importantly potential users of their products. A simple inquiry can already generate a lot of information which can already be a challenge to organize. With several designers in a team, the challenge is greater to make sure that every team member has the same level of design information to be able to move on as a team.

Please reflect on the following fictive situation...

The kick-off meeting with clients Speeda Inc. finally happened yesterday. A team of 6 designers/engineers are now enlisted in the project of designing a new breed of bicycle baggage transporter. Designers Alice and Bob are immediately assigned some tasks by the project manager Mike. Today Alice is going to visit an exhibition of bicycle latest technology which coincidentally happens in a good time- to find out the market situation. While Bob is going to observe/interview buyers at one reputable bicycle store in town, and hopefully get some users who have suitable profiles and are willing to participate later on in their research...

After a long day, Alice and Bob come back with a lot of information. They have taken along notes, photos, brochures, etc. Both Alice and Bob are wondering how they can organize this information neatly and share it quickly with their team members. They try to ask the team for a quick meeting, but it's difficult to get everyone together especially at this moment when everyone is busy doing field studies. Preparing a document could be a good idea to share the info with the team, but it takes time especially with the different (physical) media of information that has been collected. Alice and Bob just want to "drop" their findings into a common place that everyone can refer. This way, everyone can access the information him/herself when he/she has time.

Question: Do you find this challenge familiar in your work setting?

Your answer: ...

Question: Please indicate (with an ' $\mathbf{X}$ ') all statements that are relevant to your current documenting practice.

\begin{tabular}{|c|c|c|c|c|}
\hline $\begin{array}{l}\text { We document } \\
\text { as deliverables } \\
\text { to third parties }\end{array}$ & $\begin{array}{c}\text { Our } \\
\text { documents } \\
\text { are mostly } \\
\text { formal reports }\end{array}$ & $\begin{array}{l}\quad \text { We document } \\
\text { (..... before) } \\
\text { (..... as ) } \\
\text { (..... right after ) } \\
\text { we perform something } \\
\text { OR } \\
\text { (..... before we forget } \\
\text { that we have done } \\
\text { something) }\end{array}$ & $\begin{array}{l}\text { Every team } \\
\text { member has } \\
\text { equal right to } \\
\text { access and } \\
\text { modify the } \\
\text { documents }\end{array}$ & $\begin{array}{c}\text { Designers use } \\
\text { our } \\
\text { documents to } \\
\text { guide design } \\
\text { activities }\end{array}$ \\
\hline
\end{tabular}

Your additional remark: ...

\section{Proposed Functionality 1:}

Imagine a different situation...

Alice returns from her field visit to a bicycle fair in Utrecht. She has made many contacts with bike manufacturers at the fair. She's quite satisfied with what she has learned of the latest bicycle-related designs and technologies. During the fair, she had a chance to observe the 
state-of-the-art of bicycle baggage transporters. She took many photos that highlight their main features so she could show and discuss them with the team. She also took some brochures to get references/contacts of the companies... Now that she's back in the office, she wants just to put all information she has just learned quickly and call it a day. Alice uploads the photos she shot to the company's server where everyone with a login can access. But she's not done yet; she wants to give out her reviews and opinions now that they are still fresh in her memory. She opens her internet browser and runs an application called "Scenario Central". She finds the photos she just uploaded, gives them short descriptions and annotates some parts of the photos.

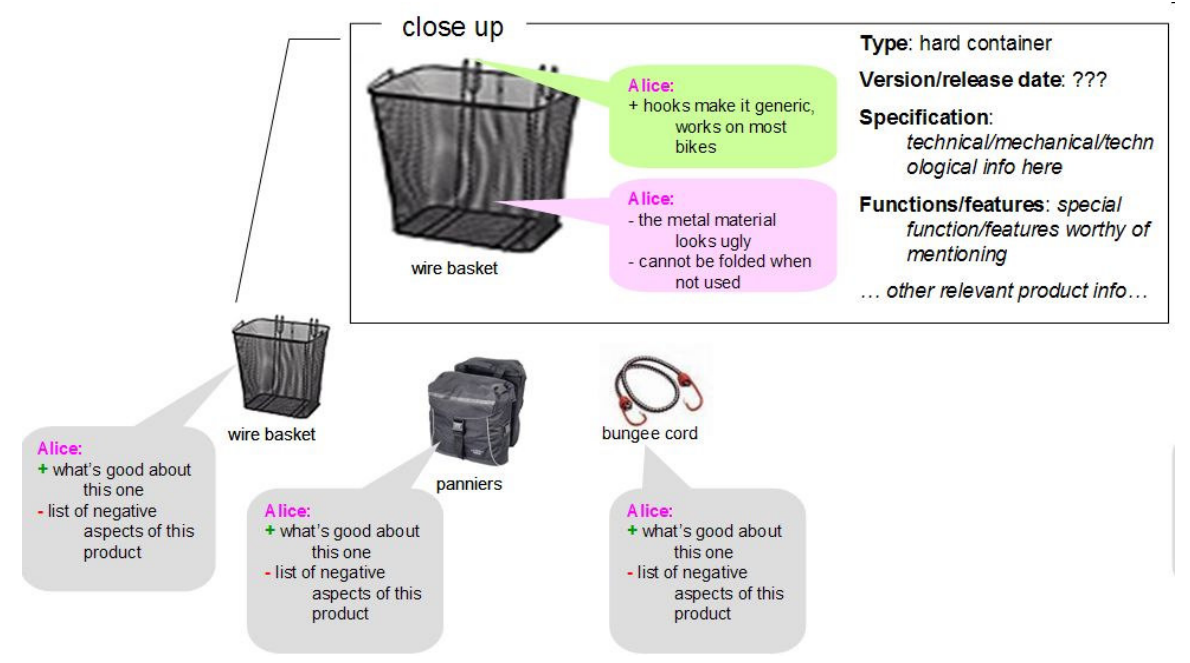

Figure 11-2.1: An overview of existing products with comments and annotations.

Question: Do you think this functionality of collaboratively building knowledge of existing products can be useful in your organization?

\begin{tabular}{|l|l|l|c|}
\hline I don't agree & Neutral & I agree, with some changes & I completely agree \\
\hline
\end{tabular}

Your explanation: ...

Bob came back from surveying the bicycle stores a bit later after Alice left for home. He checks the application "Scenario Central" to get a glimpse of what Alice has put there. Aha! Bob reads Alice's positive review about product $X$ that she found at the fair. Coincidentally, today Bob met a user who has been using product $X$ for some time and is not satisfied with it. Bob immediately put his findings as a reply to Alice's review... He then continues with registering the information about existing products and users he found in his observation. On the same work area, Bob adds 3 user profiles he has had interesting conversation with. Each of them has experiences and strong opinions about the existing products. "This kind of users will be valuable information source in this project", Bob thinks. Bob connects each user profile with product(s) they have used so far, along with the comments these users have made (which are on Bob's notes). Bob knows he still needs to give a more thorough and structured review about these users and products, but for now this is sufficient just so that he remembers the key details. 


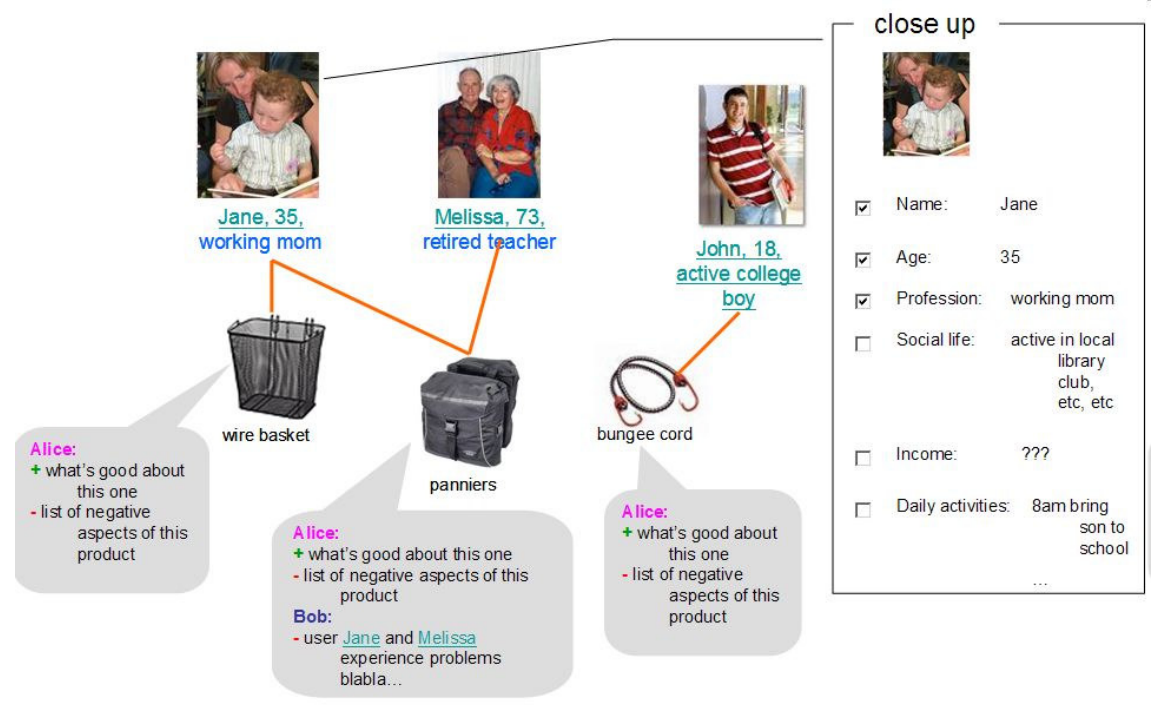

Figure 11-2.2: An overview of users and products they use.

Question: Do you think this functionality of visualizing user profiles and products they use can be useful in your organization?

\begin{tabular}{|c|c|c|c|}
\hline I don't agree & Neutral & I agree, with some changes & I completely agree \\
\hline
\end{tabular}

\section{Your explanation: ...}

\section{Input from Your Current Practice 1:}

The proposed functionality is still in idea phase. To be able to implement it, I need to learn from your current practice on the following activities.

\section{Getting to Know the Users}

Question: Do you create fictive personages or real user profiles?

Your answer: ...

Question: When you are documenting your research results about users, what aspects (of the users) are you thinking about? If you use specific media to capture certain aspects, please also mention them. E.g. contact information (text), physical appearance (photos/videos), daily life (schedule/schema) and so on.

\section{*Your answer:}

\section{Learning from Existing Products (Competitors) and Latest Technology}

Question: When you are documenting your research results about competitor products, what aspects (of the products) are you thinking about? If you use specific media to capture certain aspects, please also mention them.

\section{*Your answer:}

\section{Challenge 2:}

Design information becomes meaningful when it is interrelated. One way to make sense of the information is by combining information pieces together to drive the creation of scenarios. For 
example, information about a product and a user could have more meaning in scenarios about how the user interacts with the product in certain situation.

Please imagine the following situation...

Bob has made an appointment to visit a user. Jane the user is in mid-thirties and a single-parent with a 4 year-old son Ben. Jane is busy as she has to manage her work activities, social life and a young son. As an idealist and sport enthusiast, she bikes nearly everywhere: to bring her son to school, to work, or just to enjoy biking and picnic on weekends with Ben. Bob asks Jane to show and tell how and when she uses each one of her bike accessories, while he is videotaping. For different activities, Jane uses a combination of products that she feels best in certain situations. For example, when she's going to work, she would install a different set of products than if she's going on a picnic with Ben, etc...

Bob uploads the video to the server. He is somehow sure that his colleagues will need his guidance to find out the interesting parts of the video. Otherwise, watching a whole 47- minute long video is too much to ask from his fellow designers...

Question: In which occasion and for what reason do you have to flesh out user stories?

Your answer:

Question: To which audience and using what media do you communicate the user stories or scenarios? E.g. fellow designers (oral, role play), users participating in testing (narrative and pictures), etc.

\section{Your answer:}

\section{Proposed Functionality 2:}

Imagine a different situation...

Bob has now got an overview of the bike-related products around Jane's life. When he reaches his work desk, he immediately uploads the video to the server. He then opens "Scenario Central" application, and finds the video he just uploaded. Bob enters a description for the video, and then goes through it. At some interesting frames, he pauses the video and enters a few more comments, focusing on problems that user Jane encounters with her current products.

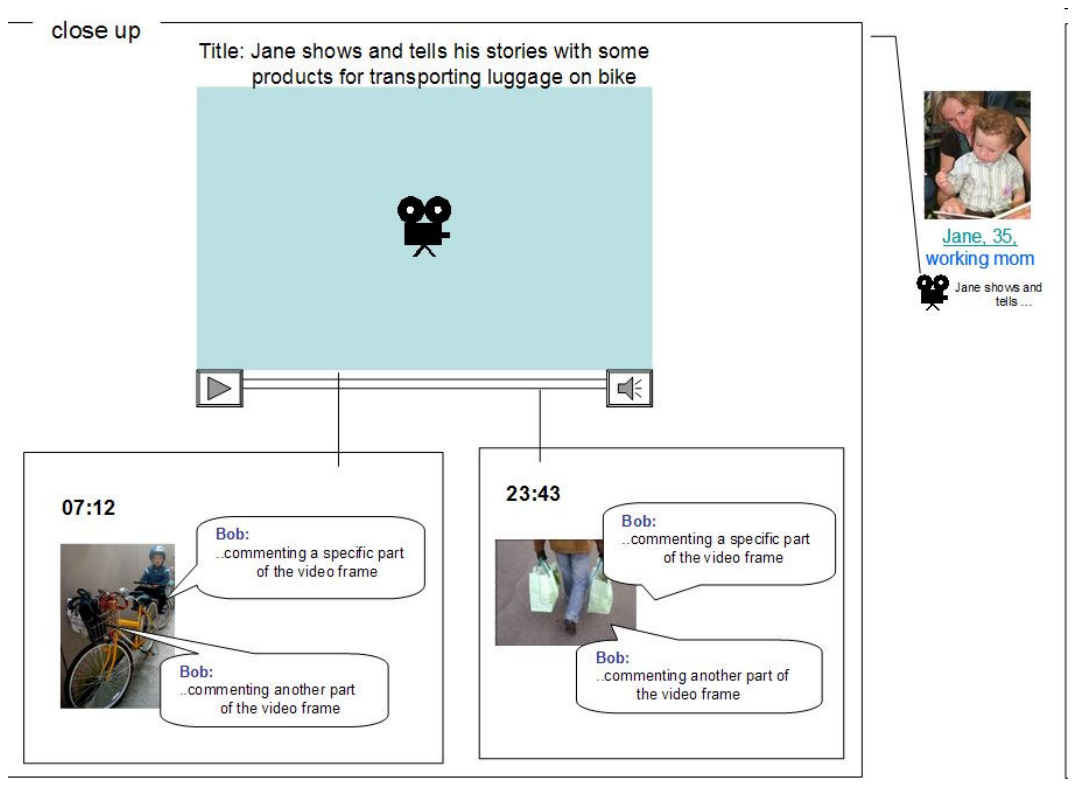

Figure 11-2.3: Observation videos can be annotated to mark important parts. 
Question: Do you think this functionality can be useful in your organization?

\begin{tabular}{|l|l|l|l|}
\hline I don't agree & Neutral & I agree, with some changes & I completely agree \\
\hline
\end{tabular}

Your explanation: ...

Bob decides to work on his analysis further. He specifically notices the diverse goals and situations Jane has concerning transporting "something" on her bicycle. Bob lists Jane's goals and relates the situations that can be related to each goal. These goals and situations can be used later on as inspiration for other scenarios concerning different users. For actions that Jane perform, Bob can indicate her emotions using emoticons, e.g. installing the toddler-seat is no fun for Jane thus a grim face next to it.

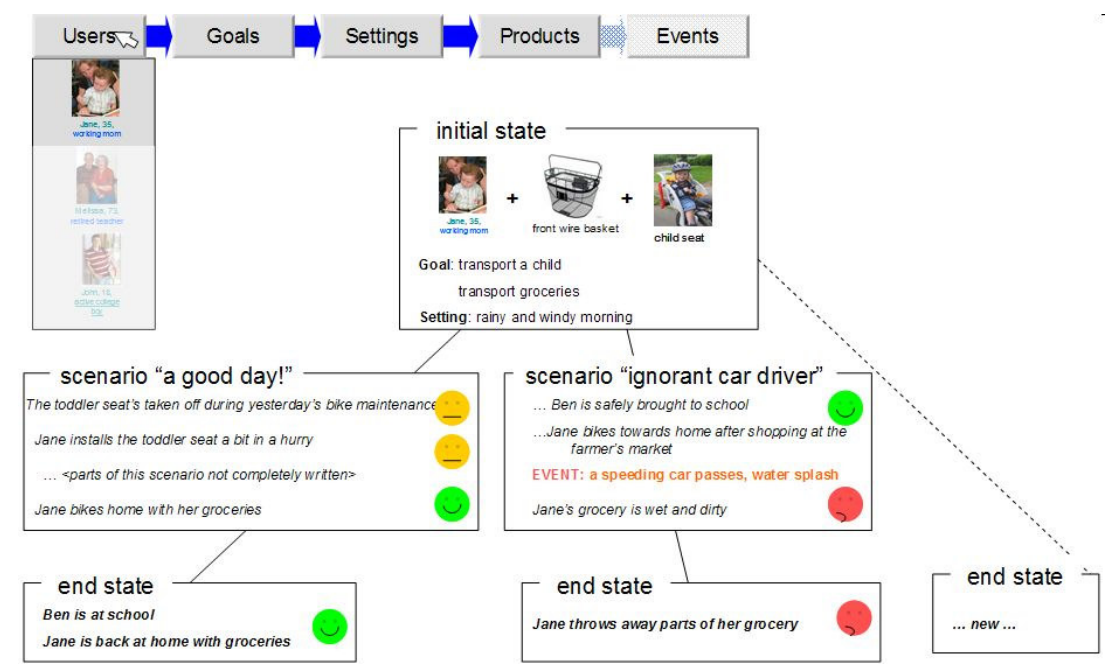

Figure 11-2.4: Scenario building toolbox consists of selectable elements. Notice that Jane's scenarios can

be based on observation (left) or based on plausible event imagined by designers (right), altogether compiling an overview of Jane's use of existing products.

Question: Do you think this functionality of documenting scenarios can be useful in your organization?

\begin{tabular}{|l|l|l|l|}
\hline I don't agree & Neutral & I agree, with some changes & I completely agree \\
\hline
\end{tabular}

Your explanation: ...

Input from Your Current Practice 2:

Understanding Use Situations (Use and problem)

Question: When you are documenting your research results about users and their product use situations, what aspects (of the use situations) are you thinking about? If you use specific media to capture certain aspects, please also mention them. E.g. user goals, where the situation takes place (photos/videos), what does the user do (notes), what happens, etc.

*Your answer:

\section{Challenge 3:}

Design knowledge about users and use situations tends to grow, creating a challenge to organize it. When we have such a broad range of knowledge, how do we understand it enough to make it useful to our design process? I could imagine that a piece of knowledge about 
users/products could be an important decision factor, and sometimes need to be pulled to the surface so that other decision-makers know about it.

Please imagine this situation...

The "bike luggage transporter" design team is meeting for the first time after the kick-off meeting. During this period, everyone has been busy doing research (desk research or field studies). Therefore, this meeting aims to be a forum where everyone can share what he or she has found during the research. And of course, if there's time left, the team can discuss what they must do now, how to move on, etc.

Before the meeting, designers (individual or in group) prepare presentations to describe their findings within 10-15 minutes time frame. Most often, this is nowadays done using Powerpoint presentation which will be quite tedious to manage afterwards. Quite often, time runs out before any meaningful discussion gets to the table. When this happens, Mike the project manager (as a representative \& member of the design team) and other management will have another meeting, and later on decide what to do next...

Question: Within your organization, are key members of the design teams involved in decisionmaking? E.g. concrete decisions such as 'are we going to observe more users?' or 'shall we use that technology by company $X$ ?'.

\begin{tabular}{|c|c|c|c|}
\hline Not at all & Rarely & $\begin{array}{c}\text { Only in some design projects } \\
\text { (please explain) }\end{array}$ & Always \\
\hline
\end{tabular}

Your explanation: ...

Question: Does every team member know the reason why he or she has to perform a specific task?

\begin{tabular}{|c|c|c|c|}
\hline Not at all & Rarely & $\begin{array}{c}\text { Only in some design projects } \\
\text { (please explain) }\end{array}$ & Always \\
\hline
\end{tabular}

\section{Your explanation: ...}

\section{Proposed Functionality 3:}

Imagine a different situation...

The "bike luggage transporter" design team is meeting for the first time after the kick-off meeting. During this period, everyone has been busy doing research (desk research or field studies) and now the "Scenario Central" application shows a good overview of the users and products they currently use. Among these, some user-product relationships have been extended into different versions of scenarios. Mike the project manager has asked everyone to get acquainted with all the information posted on "Scenario Central". The meeting will discuss what to do next as a team, instead of explaining the design information (which is already registered in "Scenario Central") to one another. 


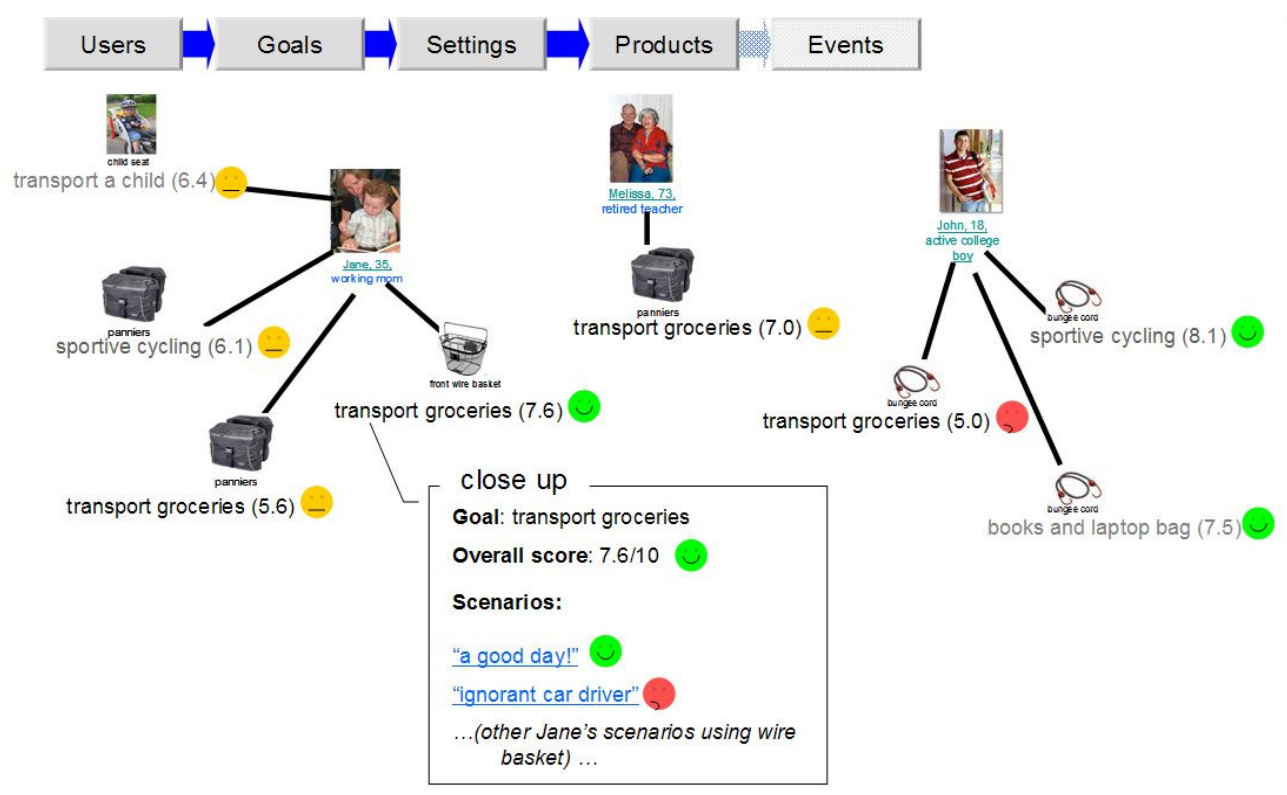

Figure 11-2.5: An overview of users and their goals using specific products, each goal is scored (number and emoticon). The goal "transport groceries" is darkest because it is the most common goal among the users. A close-up into a user goal reveals several relevant scenarios that have been registered.

Question: Do you think this "scenario overview" functionality can be useful?

\begin{tabular}{|c|c|c|c|}
\hline I don't agree & Neutral & I agree, with some changes & I completely agree \\
\hline
\end{tabular}

\section{Your explanation: ...}

During the meeting, designers are 'empowered' with the well-organized information that they can easily refer to specific scenario(s) to back up their opinions. Mike suggests a discussion on the user goal "transporting groceries" because it looks promising as a tentative direction. The "Scenario Central" application has a function to filter scenarios based on a specific element. To aid their discussion, Mike uses the filter function to show only information relevant to the goal "transporting groceries". The designers see the overview of problems with current products when their users "transport groceries", and this helps them to focus.

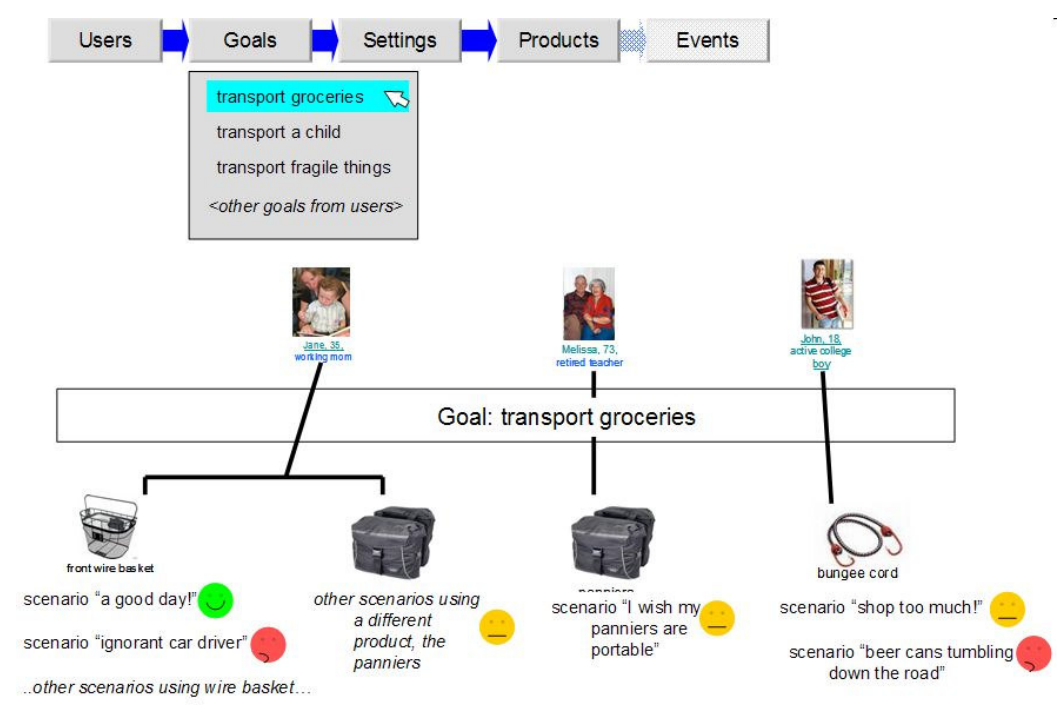

Figure 11-2.6: An overview of the goal "transporting groceries", showing the users that share this goal and their use scenarios. 
User Jane has elaborate scenarios in different use aspects. She also has the most experience with different products. Bob proposes that the team starts by getting to know this user. The filter function comes in handy to display only scenario overview about Jane.

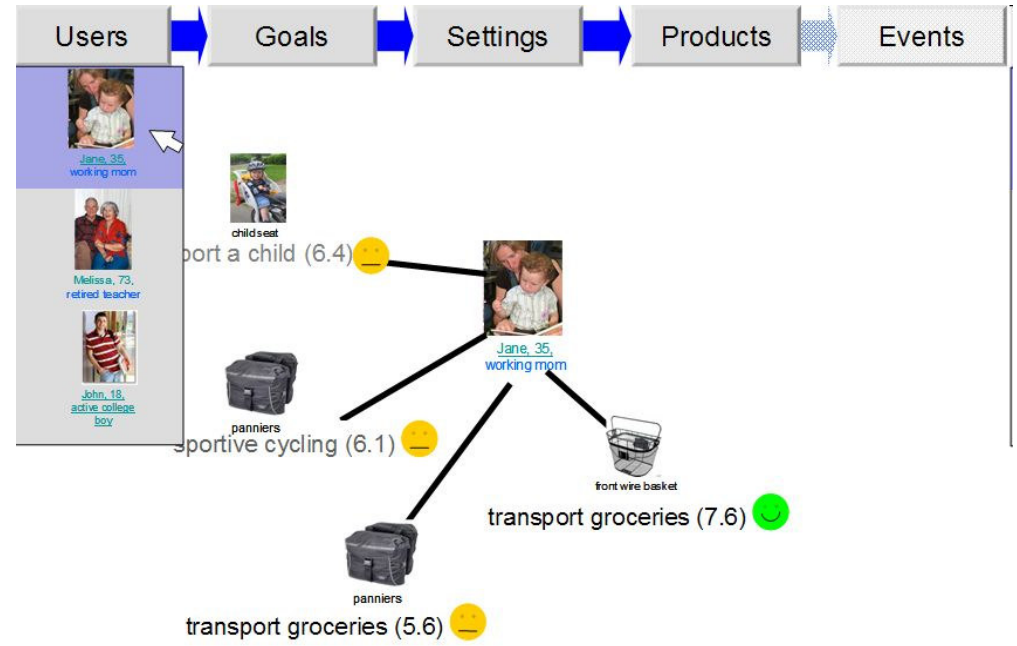

Figure 11-2.7: An overview of user Jane, the products she uses and her goals. User Jane in the dropdown is highlighted blue, meaning that the overview is filtered to only show Jane.

Question: Do you think this "scenario filtering" functionality can be useful in focusing on specific issues?

\begin{tabular}{|c|c|c|c|}
\hline I don't agree & Neutral & I agree, with some changes & I completely agree \\
\hline
\end{tabular}

Your explanation: ...

During a brainstorm session, the design team quickly comes up with many ideas. A rough concept $\mathbf{X X X}$ is created: some sketches are drawn, specific features are proposed, and to-do list is created (i.e. required further studies to verify that the proposed concept is feasible). Of course the design team does not forget to imagine how the user would use XXX; it's user-centred design after all. The team chooses to try XXX in Jane's life situation to see how it would perform (i.e. how pleased would Jane be using it?).

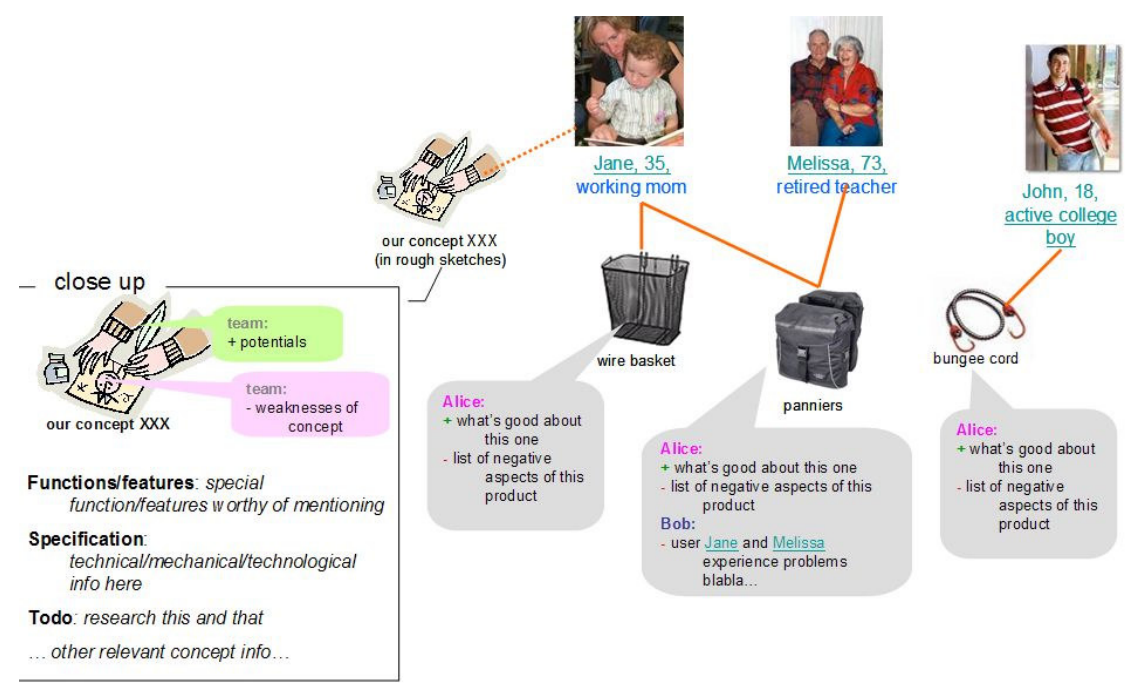

Figure 11-2.8: A new concept $X X X$ is proposed and will be put into hypothetical use by user Jane (the dotted orange line). 


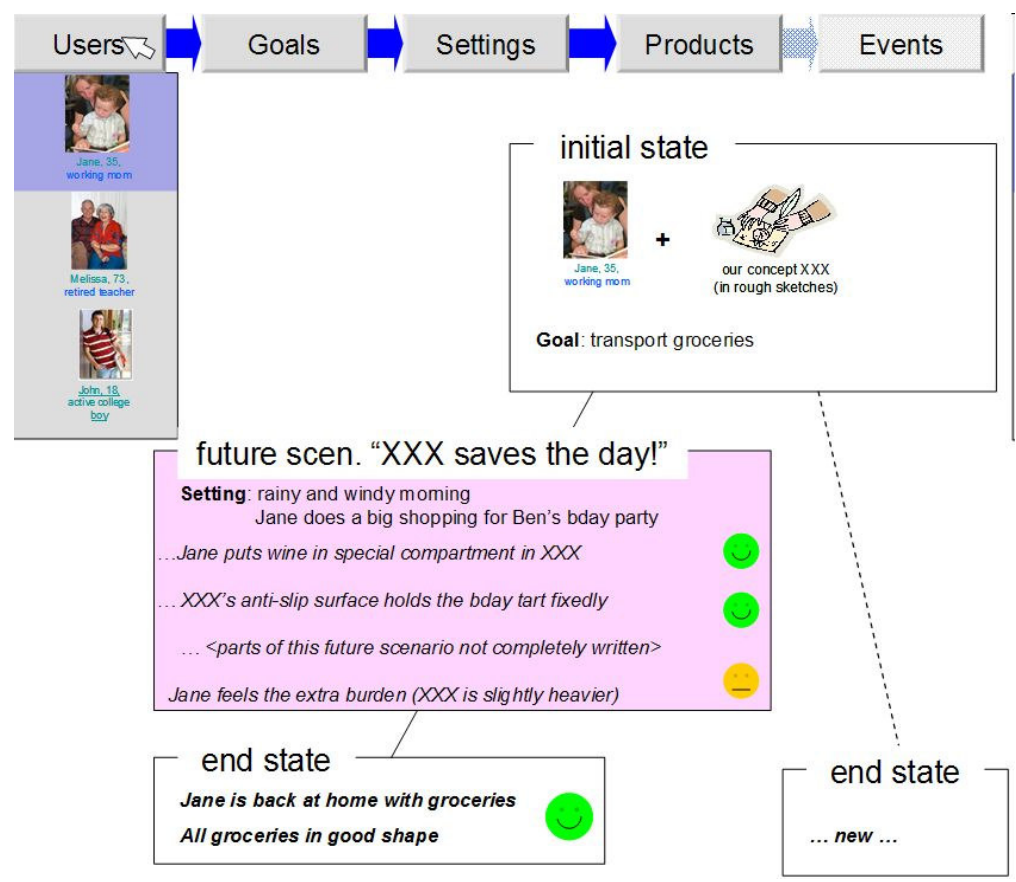

Figure 11-2.9: The concept XXX within a hypothetical use by user Jane, helping designers to imagine the

\section{future scenarios.}

Question: Do you think the functionality to create scenarios about "future use of a concept" can be useful?

\begin{tabular}{|c|c|c|c|}
\hline I don't agree & Neutral & I agree, with some changes & I completely agree \\
\hline
\end{tabular}

\section{Your explanation: ...}

Some days later, Bob and Charlie have been working together to refine XXX. Despite it seemed near perfect in the beginning, they still change many parts of concept XXX. Luckily the "Scenario Central" application helps them to keep track what they are doing; it indicates to them other parts of XXX and scenarios that might need to be adjusted.
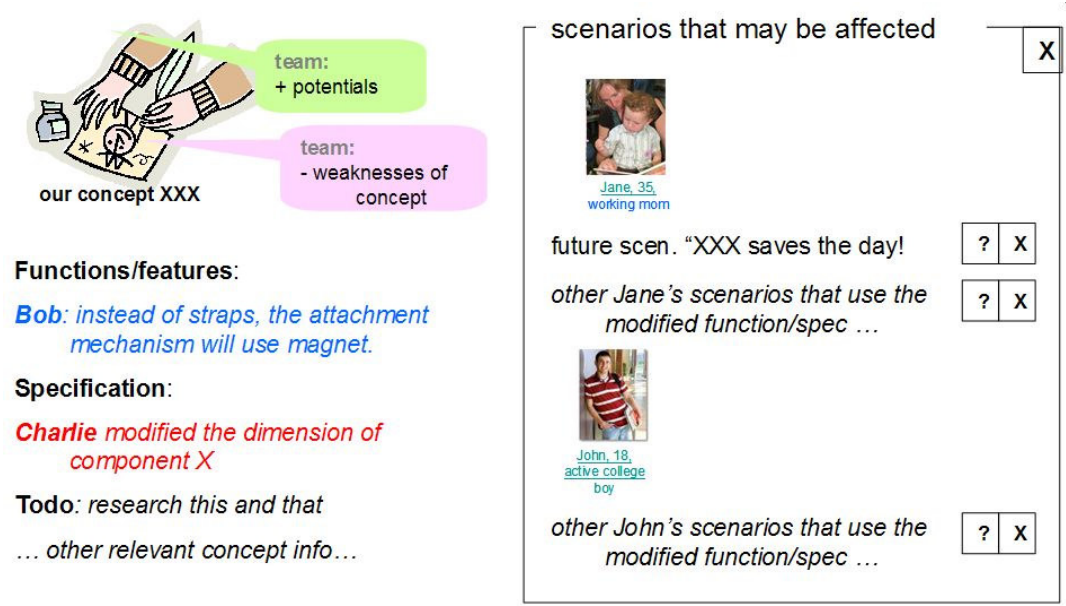

Figure 11-2.10: Changes on concept XXX might have further consequences. The tool reminds designers of them.

Question: Do you think the functionality to keep track changes in products and in scenario plots can be useful?

\begin{tabular}{|l|l|l|l|}
\hline I don't agree & Neutral & I agree, with some changes & I completely agree \\
\hline
\end{tabular}


Your explanation: ...

\section{Input from Your Current Practice 3:}

Question: In the early concept stage, what media do you use to represent your ideas (or concepts)?

Your answer: ...

Question: When you need to document an idea (or concept) for later use, what aspects/information do you register to make sure you remember it? E.g. physical appearance, functions, technology used, peer comments, critiques, etc.

Your answer: ...

Question: When changes to a concept are made, how do you keep track of the consequences (i.e. knowing what will be influenced by the changes)?

Your answer: ...

Last Question: Is there any functionality that you miss?

Your answer: ... 


\section{Appendix 3 The Usability Workshop Program}

Design for Usability Symposium, Delft, 12 Nov 2009

Audience: all-round product designers, project managers, design students

The workshop introduces the basics of design for usability by means of a lecture and some group assignments. Participants will experience how to define approaches regarding usability related design assignments. Additionally they will be exposed to a new way on managing design information.

Group assignment: How to manage design information (duration 1 hour)

Goal: Using a content \& scenario management tool, participants learn to process user-centred design data into meaningful scenarios.

\section{Assignment steps:}

Step 1: Introduce a made-up persona and other design data. Present the scenario tool and show the functionality to manage these data (max. 15 minutes).

Step 2: Participants brainstorm possible result data from executing their approach (in groups). Each group creates 1-2 distinct personas (fictive personification of users), thinks up their characteristics and goals. Participants are guided to identify other scenario elements and to think of possible scenarios.

(Each group is provided with a laptop with access to the scenario tool. Each group is given flexibility to either work on paper first or directly on the tool. Organizers walk around and help them with questions.)

Step 3: Each group presents their results in a 5 minute plenary presentation what they have done using the tool, and how they think it could be useful in their projects/companies.

(Each group needs to connect their laptop to the projector. Consider to start videotaping from the presentations until the end.)

Step 4: Short feedback session: on usefulness of the functionality, what can be added and/or improved. 



\section{Appendix 4 Evaluative Questionnaire (Intermediate and Final Evaluation)}

Thank you for participating in this questionnaire. We kindly ask for your personal details in case we need to clarify some answers.

Name:

Email:

Please briefly describe your position at [the company]:

First of all, for the researcher to understand your responses better, please answer these two open questions.

1. Please mention products/projects in which you could imagine yourself (as part of a team) using the tool during the design process

2. Please also mention the ones that you think would NOT be supported by the tool at all

Please rate your agreement with the following statements about how you feel in general when using the scenario tool. You may write additional comments to elaborate your answers. If you need more space, please use the back of the page and number your comments.

\section{A. Functionality (based on the working prototype)}

1. The tool covers the necessary design information (i.e. everything you need to document in a project) Final evaluation: The tool covers the necessary information for scenario building in a design project

Strongly Disagree 1----2----3----4----5----6----7 Strongly Agree

2. The tool covers the necessary functionality for creating and using scenarios within design projects

Strongly Disagree 1----2----3----4----5----6----7 Strongly Agree

NA

3. The tool gives a useful overview of the documented data.

Strongly Disagree 1----2----3----4----5----6----7 Strongly Agree

NA

4. *It has the functions and capabilities I expect it to have (see also section E) Strongly Disagree 1----2-----3----4----5----6----7 Strongly Agree

\section{B. Ease of Learn \& Use}

1. I can learn to use the tool on the go

Strongly Disagree 1----2----3-----4----5----6----7 Strongly Agree

2. The categories for information are self-descriptive (i.e. actors, events, scenarios, requirements, etc)

Strongly Disagree 1----2----3----4----5----6----7 Strongly Agree

3. Documenting information with the tool is intuitive

Strongly Disagree 1----2----3----4----5----6----7 Strongly Agree

4. The tool presents logical relationships between information Strongly Disagree 1----2----3----4----5----6----7 Strongly Agree

5. *I can easily access the information (that I have documented)

Strongly Disagree 1----2----3----4----5----6---7 Strongly Agree 
6. *I don't notice any inconsistencies (as I am using it)

Strongly Disagree 1-----2----3----4----5----6----7 Strongly Agree NA

7. * can effortlessly (quickly and easily) accomplish what I want to do with it Strongly Disagree 1-----2----3----4----5----6----7 Strongly Agree NA

\section{Usefulness (fittingness)}

1. The tool improves the organization of design information

Strongly Disagree 1----2----3----4----5----6----7 Strongly Agree NA

2. *Documenting information with the tool is disruptive to my work Strongly Disagree 1-----2----3----4---------6----7 Strongly Agree NA

3. The tool could support collaborative building of design knowledge in my project team Strongly Disagree 1----2----3----4----5----6----7 Strongly Agree NA

4. *The effort I spend using the tool is worth the benefits Strongly Disagree 1----2----3----4----5----6----7 Strongly Agree NA

5. It makes the things I want to accomplish clearer and easier to get done.

Strongly Disagree 1----2----3----4----5----6----7 Strongly Agree NA

6. The tool contains features that support discussions with my fellow designers or with others Strongly Disagree 1----2----3----4----5----6----7 Strongly Agree NA

7. Overall, I expect the tool to be useful in my organization Strongly Disagree 1----2----3----4----5----6----7 Strongly Agree NA

\section{Satisfaction}

1. I would recommend it to a colleague.

Strongly Disagree 1----2----3----4----5----6----7 Strongly Agree NA

2. It is fun (engaging, not boring) to use.

Strongly Disagree 1----2----3----4----5----6----7 Strongly Agree

3. I found it very cumbersome (slow, or complicated) to use.

Strongly Disagree 1-----2----3----4----5----6----7 Strongly Agree

4. It does not hinder the flow of my thinking process.

Final evaluation: *It accommodates the flow of my thinking process during analysis, synthesis and evaluation phases.

Strongly Disagree 1----2----3----4----5----6----7 Strongly Agree NA

5. It helps me to be creative (imaginative).

Strongly Disagree 1----2----3----4----5----6----7 Strongly Agree NA

6. * expect that I would use the tool frequently.

Strongly Disagree 1----2----3----4----5----6----7 Strongly Agree NA

7. Overall, I am satisfied with the way the tool works.

Strongly Disagree 1-----2----3----4----5----6----7 Strongly Agree NA

E. Additional Tool Features (based on the ideas presented using mockups) or How can the tool be improved? (final evaluation)

1. The tool should be able to give an overview/summary of the documented information. Final evaluation: The tool should give a more elaborate overview/summary of the documented information. 
Strongly Disagree 1----2----3----4----5----6----7 Strongly Agree

Which information do you want in the overview?

2. The tool should visualize the overview (thus, not only text/list).

Final evaluation: The tool should provide a more visual overview (in addition to the current textual/list overview).

Strongly Disagree 1----2----3----4----5----6----7 Strongly Agree

NA

Please explain/draw the kind of visualization you want

3. The tool should give output (e.g. printouts, text/doc files) that will be used in other design activities (in the same project).

Final evaluation: The tool should provide more types of output that will be used in other design activities in the project. (Currently the possible outputs are printouts and Excel sheet.)

Strongly Disagree 1----2----3----4----5----6----7 Strongly Agree

NA

What information and which format will be most useful as outputs in your organization?

4. Any other functionality to add? 



\section{Appendix 5 Intermediate Evaluation: Questionnaire Analysis}

The next subsections will explain the results of each category. Each subsection will address what is being evaluated in the particular category, illustrated with a box plot of the scores, and further analysis of what the scores mean. Figure 11-5.1 illustrates a box plot with some pointers on how to interpret it.

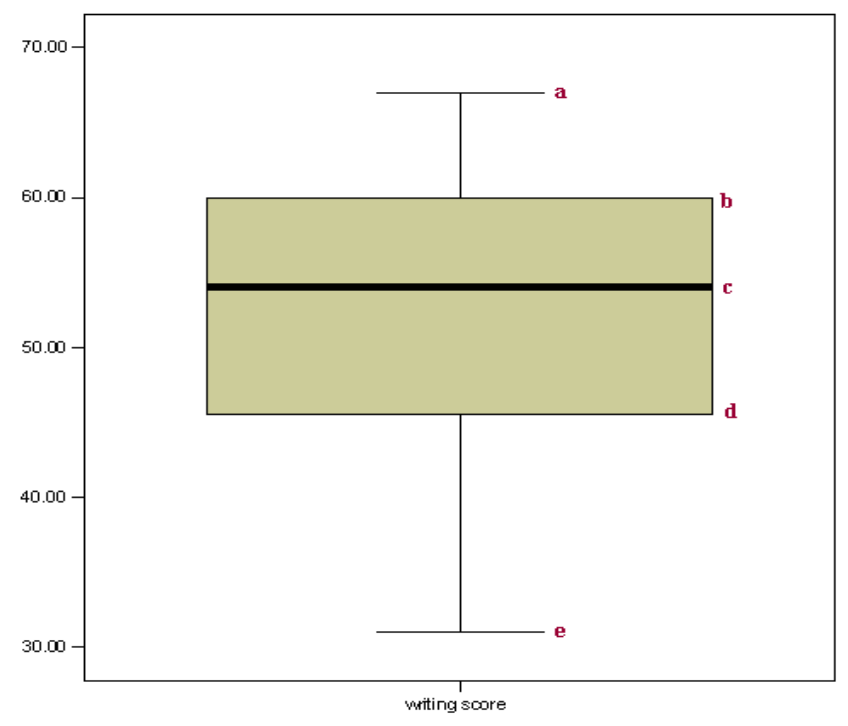

The box plot example uses data on 'writing score' (X-axis) of a group of students.

Point $a$ is the third quartile plus 1.5 times the interquartile range (the difference between the first and the third quartile).

Point $b$ is the third quartile (the 75th percentile).

Point $\mathrm{C}$ is the median (the 50th percentile), the middle value of the scores.

Point $d$ is the first quartile (the 25th percentile).

Point $e$ is the first quartile minus 1.5 times the interquartile range (the difference between the first and the third quartile).

Figure 11-5.1: An annotated example of a box plot ${ }^{8}$.

The box plot shows a box encased by two outer lines known as whiskers. The box represents the middle $50 \%$ of the data sample, which means half of all cases are contained within it. The remaining $50 \%$ of the sample is contained within the areas between the box and the whiskers. There may be extreme values that deviate significantly from the rest of the sample and they can exist above or below the whiskers of the box plot. They are called outliers, and found in the form of points, circles, or asterisks. Inside the box, there is a single line which represents the median, the middle value of the entire sample.

In the example of Figure 11-5.1, the bottom $25 \%$ of the scores would be represented by the space between ' $e$ ' and ' $d$ ', the middle $50 \%$ would be within the box (between ' $d$ ' and ' $b$ '), and the top $25 \%$ would be contained between ' $b$ ' and ' $a$ '. For this analysis, the box plots consistently use the scores from designers per question within each evaluation category as the X-axis.

\section{Functionality}

The functionality category aims to find out the coverage of the general functionality. While the prototype(s) developed during this research will not be a full-blown software package ready to use in practice, the questions pose the most important characteristics of the tool. Therefore, the questions do not only focus on the implemented functionality so far, but rather measure how much of the aimed functionality has been covered. The first three questions are straightforward questions on how well the current prototype accommodates the functions related to information documentation and processing. The last question serves as an objectivity check, by reminding the participants of other possible functionality in the later section Additional Features, and is expected to acquire low scores.

\footnotetext{
8 Image courtesy of http://www.ats.ucla.edu/stat/spss/output/descriptives.htm. Retrieved on 4 July 2010.
} 


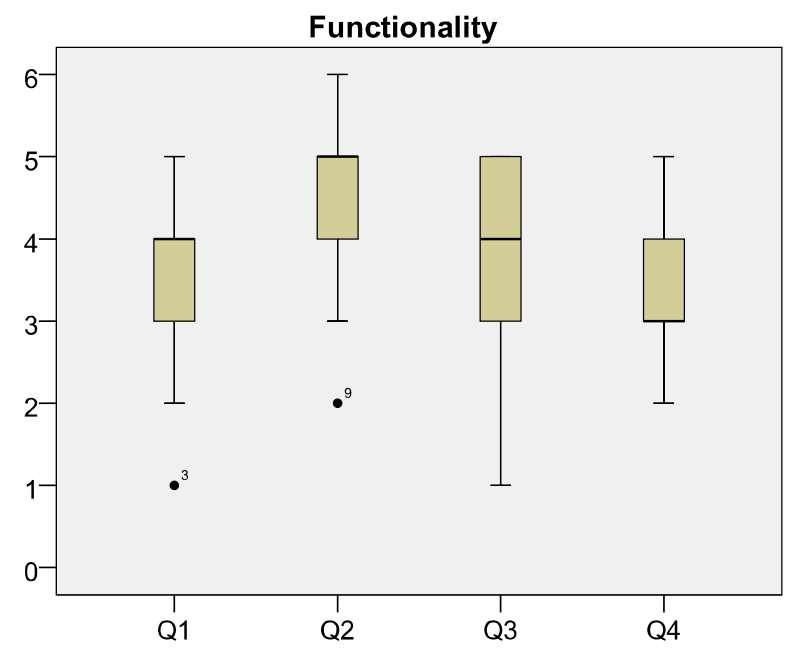

1. The tool covers the necessary design information (i.e. everything you need to document in a project)

2. The tool covers the necessary functionality for creating and using scenarios within design projects

3. The tool gives a useful overview of the documented data.

4. It has the functions and capabilities I expect it to have (see also section Additional Features)

Figure 11-5.2: The box plot of functionality scores.

Question 1 asks the designers to reflect whether the coverage is sufficient for documenting all scenario-related information. Explanations (in the questionnaire) from the designers indicate that the scope of the tool needs to be clearly defined and the formulation more precise. The support tool does not cover all design information, which in design projects also includes formal requirements, technical specifications, etc. The tool strives to support scenario creation and use in an informal manner, and therefore leaves out the formal documentation from the system. A reformulation for any future questionnaire needs to reflect this situation.

Question 2 is unambiguous, as it directly asks about the creation and use of scenarios in the design practice. The current prototype sufficiently covers the basic processing of information into scenarios.

Question 3 refers to functionality which is still work in progress during the evaluation. The current prototype gives out an overview of documented data mainly in textual tables. A more visual overview of information is in the development agenda and affirmed by the designers.

Question 4, as mentioned earlier, is put on purpose to test participants' constructive criticism.

\section{Ease of Learn and Use}

The category 'ease of learn and use' evaluates how graspable and intuitive the use of the tool is. With the informal attribute, the designers are expected to learn using the tool on the go. The first two questions are therefore the most important to find out with the current state of the prototype. The rest of the questions cannot be objectively scored with the absence of a more complete prototype. Therefore they are mainly used to trigger participants' reflection, and are considered only as an indication of the quality of the later prototype. 


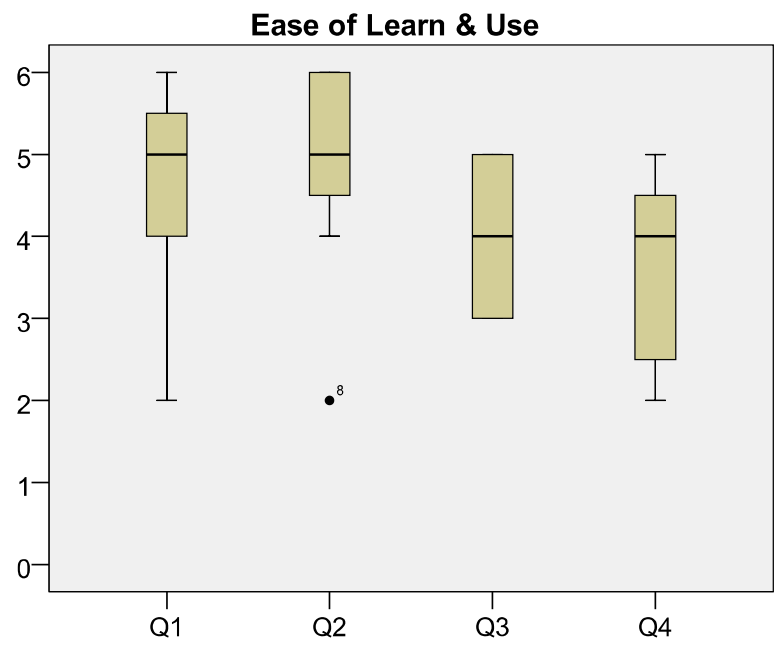

1. I can learn to use the tool on the go

2. The categories for information are selfdescriptive (i.e. actors, events, scenarios, requirements, etc)

3. Documenting information with the tool is intuitive

4. The tool presents logical relationships between information

Figure 11-5.3: The box plot of 'ease of learn and use' scores (part 1).

Question 1 and 2 are the most relevant to measure the ease of learn and use of the prototype at this stage. The relatively high scores reflect the designers' opinions that the tool layout is simple and the information categories are understandable. However, as mentioned earlier, not all of them have the same level of background knowledge about scenario-based design. This explains the large gap between the lowest score and the median for question 1.

Question 3 and 4 are limited with the capability of the current prototype. These questions will become more relevant with the final prototype, which allows the designers to try it out themselves for a better assessment.

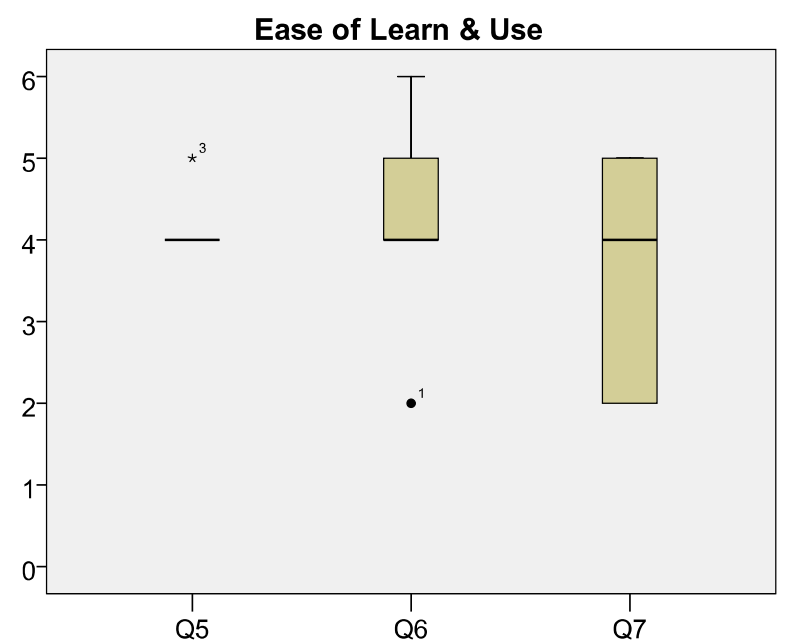

5. I can easily access the information that I have documented

6. I don't notice any inconsistencies as I use it

7. I can effortlessly (quickly and easily) accomplish what I want to do with it

Figure 11-5.4: The box plot of 'ease of learn and use' scores (part 2).

Question 5, 6 and 7 are considered too early at this stage, shown by a number of "Not Applicable' answers from the designers. Similar to question 3 and 4, answering these questions requires an intensive and extensive interaction with the prototype. For this evaluation phase, they only serve as an indication on how the tool would perform in a real work setting.

\section{Usefulness}

The usefulness category aims to qualitatively measure the added values of the tool in practice both on the organizational or personal level. It can be evaluated from two aspects. On the one 
hand, the tool can be useful on the organizational or team level, for instance by supporting collaborations between team members. On the other hand, any designer with his or her own work rituals can evaluate the tool's usefulness for individual tasks.

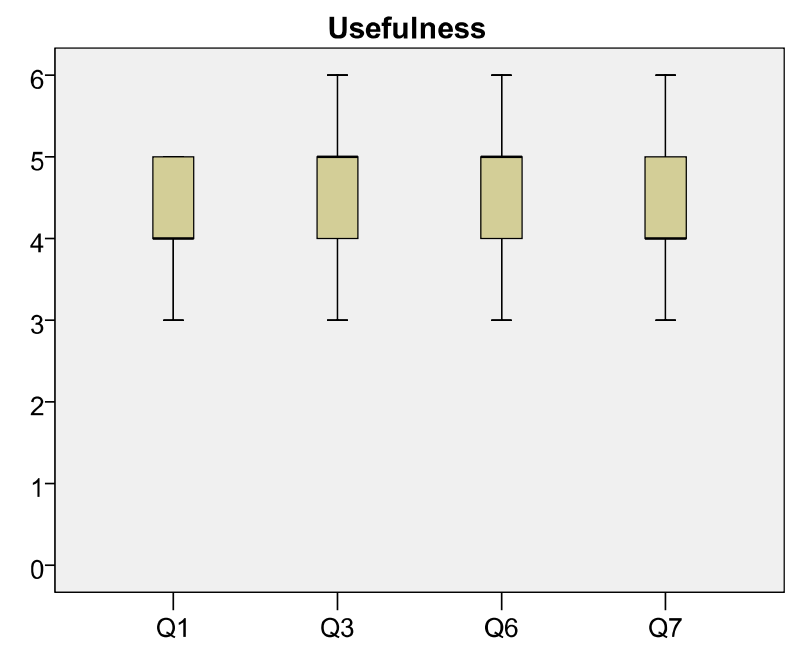

1. The tool improves the organization of design information

3. The tool could support collaborative building of design knowledge in my project team

6. The tool contains features that support discussions with my fellow designers or with others

7. Overall, I expect the tool to be useful in my organization

Figure 11-5.5: The box plot of usefulness scores (part 1).

Question 3 and 6 assess the usefulness of the tool in supporting communication and information exchange within a team. Compared with the current ad-hoc way of communicating in the company, the prototype already offers added values in organizing and viewing design information which helps designers to share information easily. The scores are a good indication on the actual practical values of the tool.

Question 1 and 7 capture the general aim of the functionality to improve the organization of design information in scenario-based approaches. The current prototype still lacks additional functionality, especially in supporting the further use of the information. Therefore these scores are expected to improve in the next phase of evaluation with the final prototype.

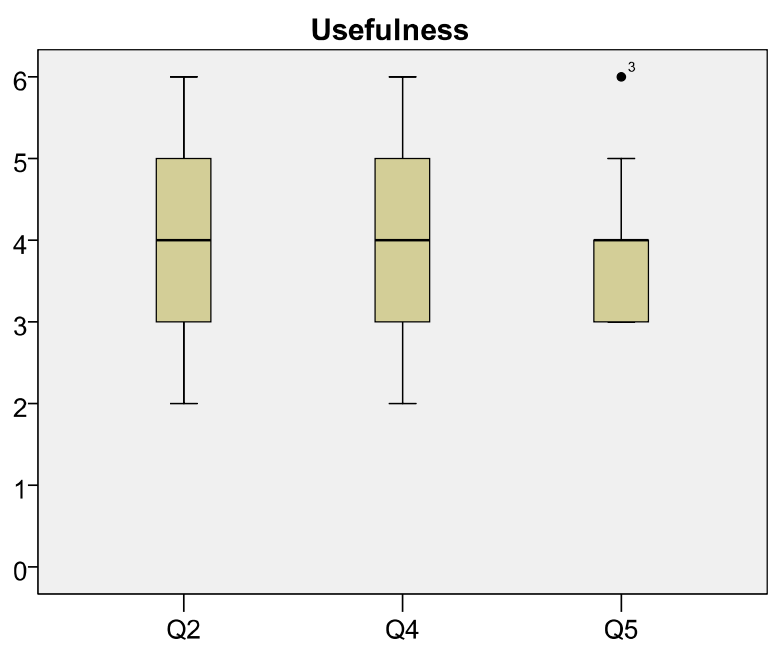

2. Documenting information with the tool is disruptive to my work

4. The effort I spend using the tool is worth the benefits

5. It makes the things I want to accomplish clearer and easier to get done.

Figure 11-5.6: The box plot of usefulness scores (part 2).

Question 2, 4 and 5 concern each designer's individual acceptance of the tool, when reflected with his or her own work ritual. As mentioned earlier, the participants have different job functions. Consequently, a wide range of results are seen depending on the type of work they perform in their functions. Nevertheless, with the next development steps aiming for a more intuitive and 
quick interaction with the tool, we expect an overall increase for the scores in the next evaluation phase.

\section{Satisfaction}

The satisfaction category aims to measure more abstract qualities and individual preferences. This category covers mainly personal opinions, yet it is important towards the designers' acceptance. The questions cover for instance likes or dislikes, the 'fun' factor, fulfillment of personal goals, and personal satisfaction.

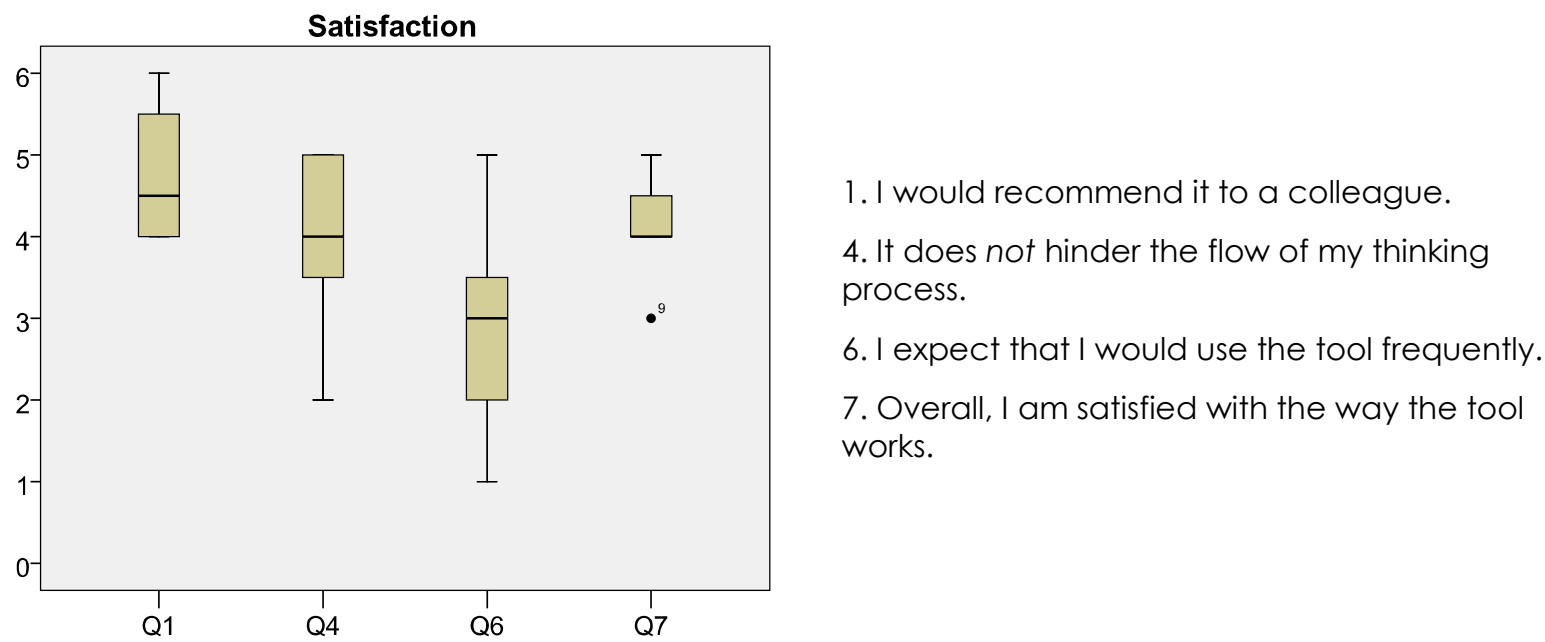

Figure 11-5.7: The box plot of satisfaction scores (part 1).

Question 4 tries to anticipate whether using the tool would cause more stress or frustration at work. The formulation however, could have been more specific and informative, for instance by pinpointing the thinking process in a particular design activity.

Question 6 faces a similar formulation problem as question 4 . The term 'frequently' is not properly defined. The time frame, in which the use is expected to take place, is also not specified. The comments from the participants indicate the reason for variable scores. They would use the tool to a very great extent at the start of the project (analysis phase), though in the later phases the use might be diminished.

Question 1 and 7 probe into the personal satisfaction and level of commitment, which are influenced by whether the designer feels helped by the tool or not. The participants generally welcome an intermediate tool that helps them process raw information (e.g. from user research, interviews, observations) into high-density information (in the form of e.g. scenarios, requirements). 


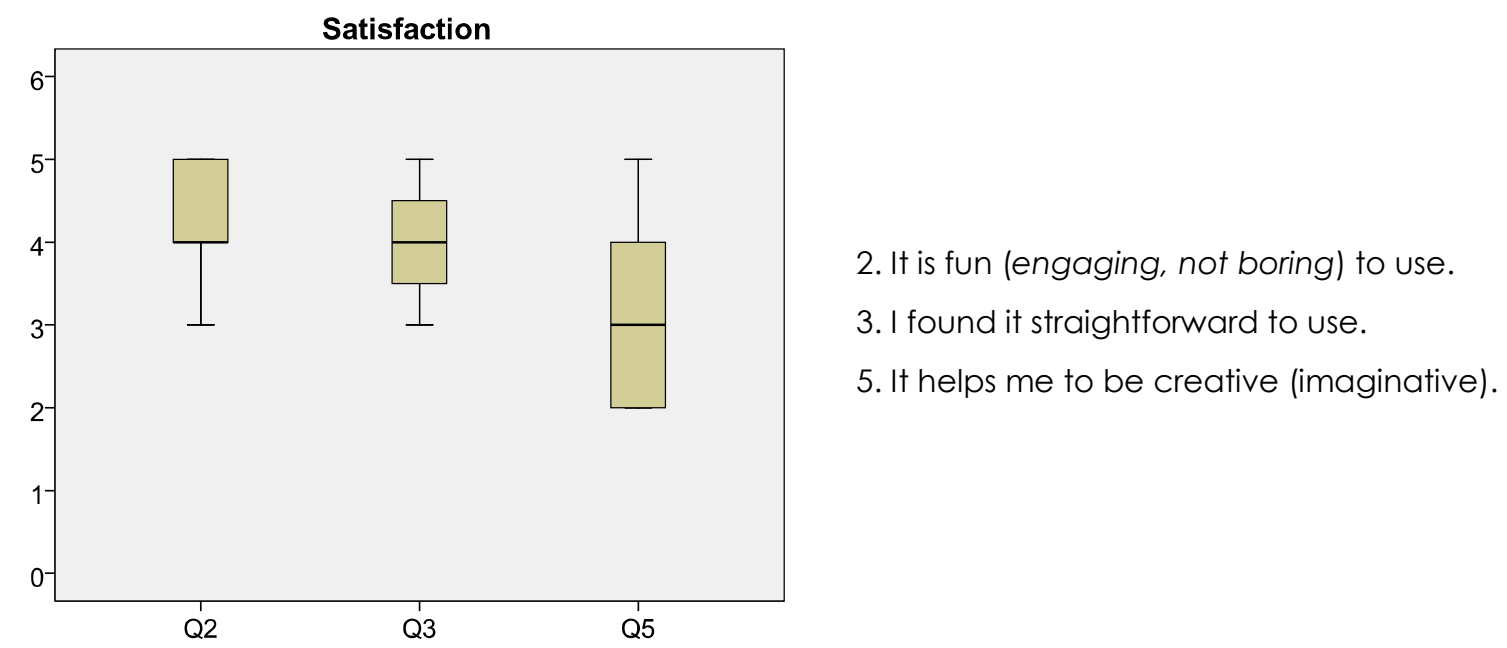

Figure 11-5.8: The box plot of satisfaction scores (part 2).

Question 3 is intentionally a negative question to check participants' accuracy in answering the questionnaires. For the analysis, the initial question "I found it very cumbersome to use" has been translated into "I found it straightforward to use". Consequently, the scores are also inverted.

Questions 2, 3 and 5 investigate the level of personal motivation for using the tool. The questions cover different aspects that could gauge this motivation level. Aiding the creative process is one aspect that is being asked in question 5. The current prototype is limited by the implementation that the interaction is too restrictive to allow the designers to use the tool for a creative process. The evaluation using the final prototype is expected to improve this aspect. On the aspect of informality, the designers prefer it since an informal tool does not oblige them to follow prescribed formal steps. The informality and non-prescriptiveness of the tool seem to influence the 'fun' factor of using it, as reaffirmed by the scores to question 2.

\section{Additional Features}

The last category aims to reconfirm the functionality that is still planned for further implementation. The questions cover the functionality, as formulated and confirmed in the earlier studies (Chapter 4 and 5), that has not been implemented in the current prototype.

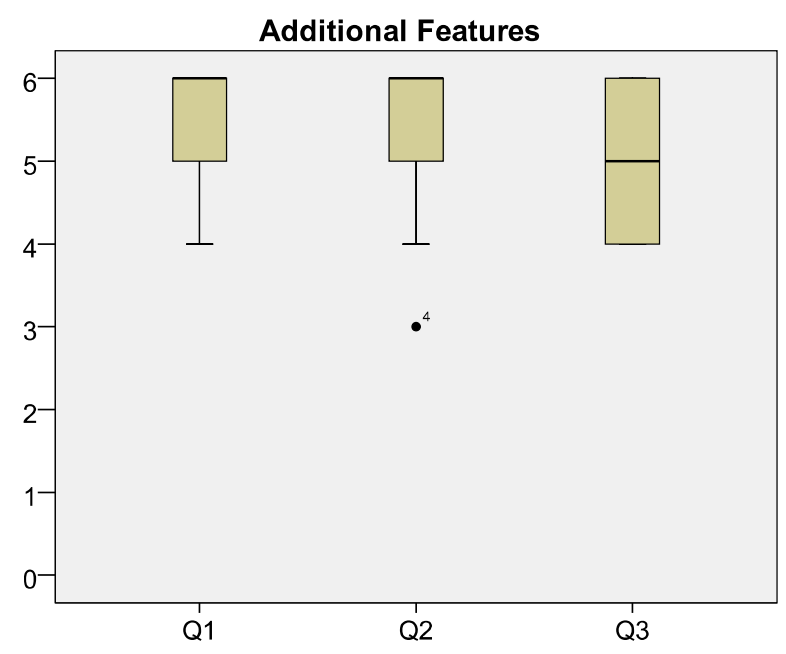

1. The tool should be able to give an overview/summary of the documented information.

2. The tool should visualize the overview (thus, not only text/list).

3. The tool should give output (e.g. printouts, text/doc files) that will be used in other design activities (in the same project).

Figure 11-5.9: The box plot of 'additional features' scores. 
This category does not measure the quality of use, and therefore is independent from other categories. The questions are rather straightforward. These questions reconfirm with the designers the functionality that is planned to be implemented in the next development steps. 



\section{Appendix 6 Final Evaluation: Questionnaire Analysis}

In brief, the participants are asked to score statements/questions between 1 ('strongly disagree') and 7 ('strongly disagree'). For the analysis, the recorded score is the actual score minus 1, so that 'strongly disagree' translates into 0 and 'strongly agree' into 6. For more explanation about the categories and the use of box plot, please refer to section 'Questionnaires' in Appendix 5. Figure 11-5.1 in particular gives a detailed explanation about what can be inferred from a box plot.

\section{Functionality}

The functionality category consists of four questions as the following:

1. The tool covers the necessary information for scenario building in a design project

2. The tool covers the necessary functionality for creating and using scenarios within design projects

3. The tool gives a useful overview of the documented data.

4. It has the functions and capabilities I expect it to have

Figure 11-6.1 shows the box plots of evaluation scores from Indes and Philips respectively.
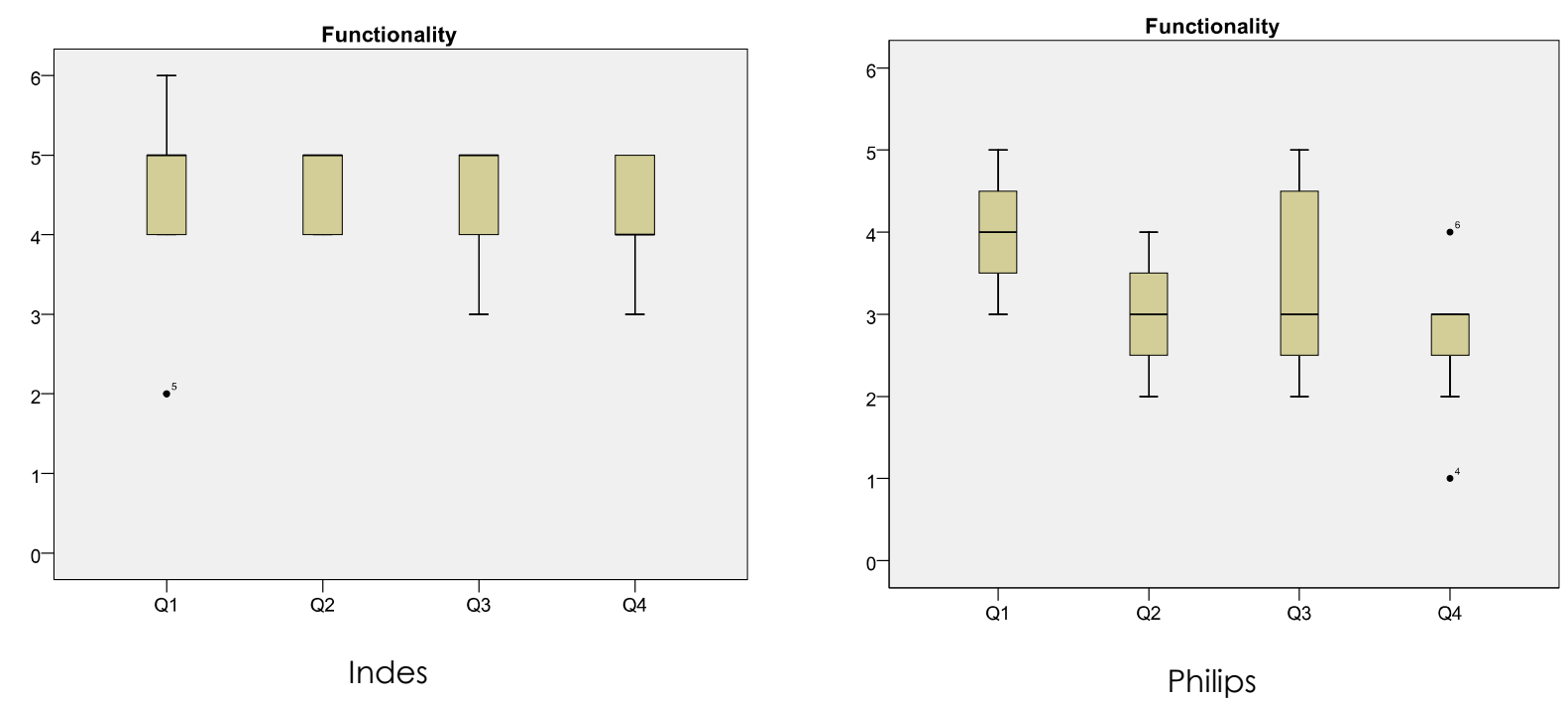

Figure 11-6.1: Detailed comparison of functionality scores between Indes and Philips.

As discussed earlier, the idea of the support tool has been developed for a medium-sized design company. The evaluation with Philips, in particular its Application Research Center (ARC), aims to explore whether the support tool is relevant to the design practice at larger companies. Overall, the lower scores from Philips reflect that the functionality of the support tool is less fitting to the practice of ARC.

\section{Ease of Learn and Use}

The 'ease of learn and use' category consists of seven questions as the following:

1. I can learn to use the tool on the go

2. The categories for information are self-descriptive (i.e. actors, events, scenarios, requirements, etc)

3. Documenting information with the tool is intuitive

4. The tool presents logical relationships between information

5. I can easily access information (that I have documented) 
6. I don't notice any inconsistencies (as I am using it)

7. I can effortlessly (quickly and easily) accomplish what I want to do with it

Questions 1 to 4 are clear enough to be answered decisively by the participants. Questions 5 to 7 on the other hand, may require some guessing because the participants have not used the tool. The participants however have been instructed to base their score using the impression they get from the demonstration. Figure 11-6.2 and Figure 11-6.3 show the box plots of evaluation scores from Indes and Philips respectively.
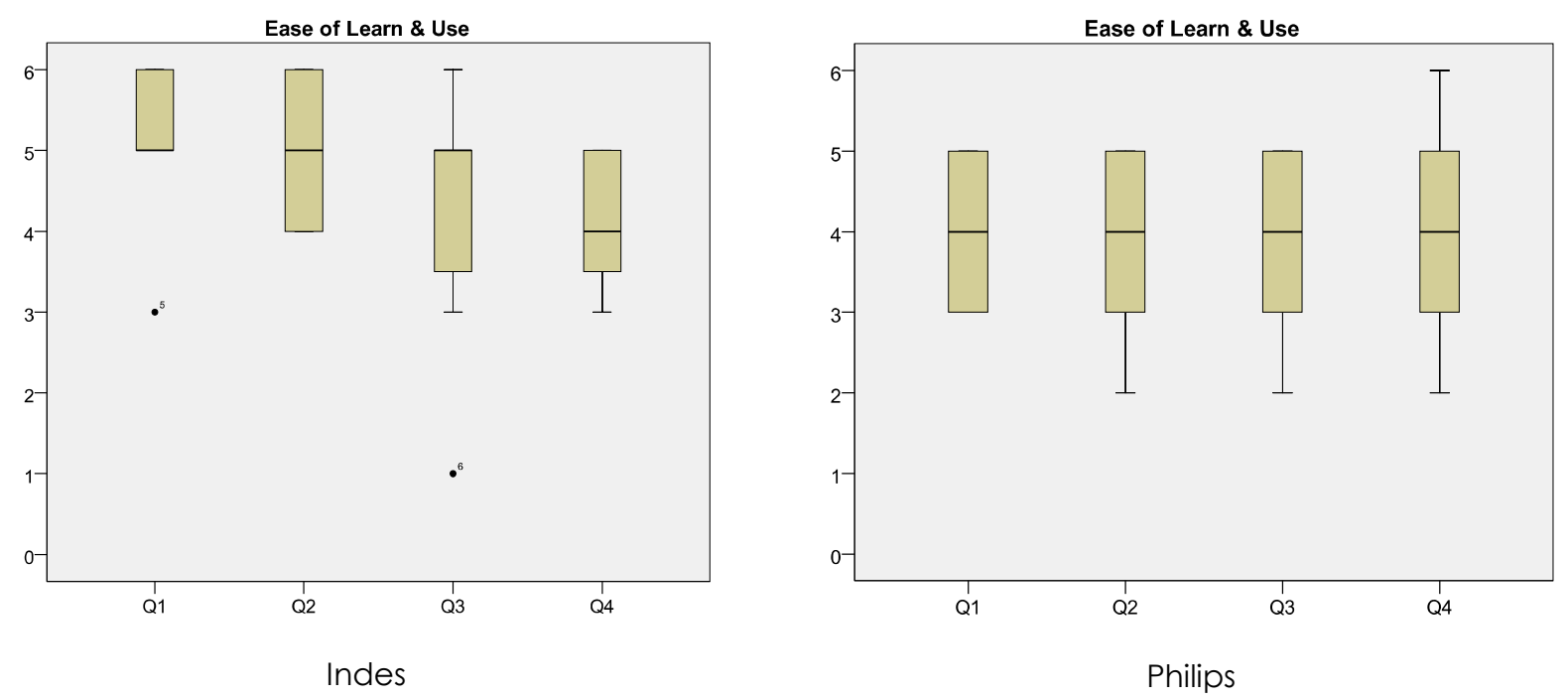

Figure 11-6.2: Detailed comparison of 'ease of learn and use' scores between Indes and Philips (part 1).

The results as shown in Figure 11.6-2 indicate that the support tool is overall easy to learn/use. Even for the Application Researchers at Philips who know the tool only from a short demonstration, the approach using the tool seems logical enough as shown that the scores are largely above the median (=3). This suggests that the participants to an extent use scenarios implicitly, and that the tool provides a semi-structure that is coherent to their thinking process.
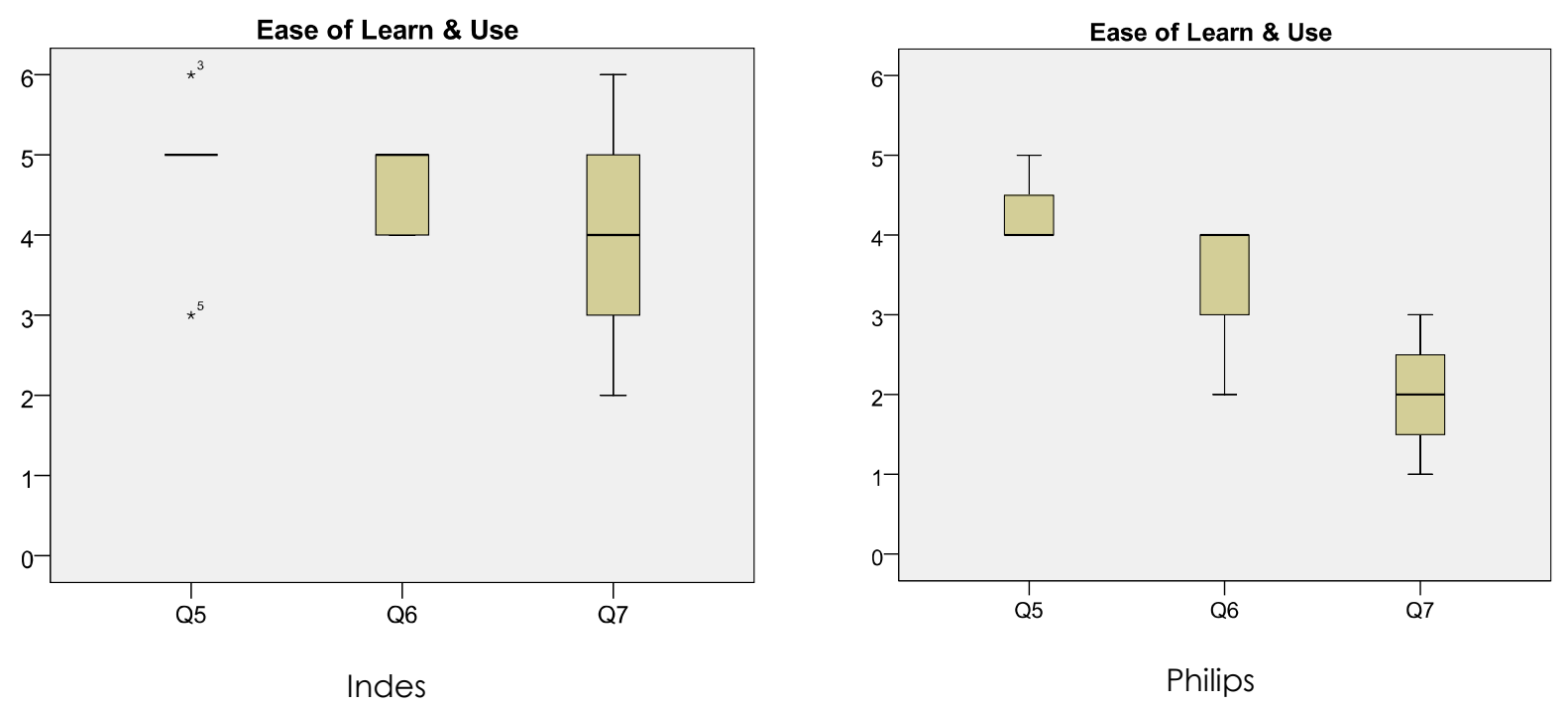

Figure 11-6.3: Detailed comparison of 'ease of learn and use' scores between Indes and Philips (part 2).

As also anticipated during the intermediate evaluation, question 5, 6 and 7 are rather challenging to answer without self-experiencing using the tool. However, they serve as an indication on how the tool would perform in the daily practice. The slightly higher scores from Indes in comparison with Philips indicate that the tool conforms better in the practice of Indes. Question 7 especially highlights this. 


\section{Usefulness}

The usefulness category consists of seven questions as the following:

1. The tool improves the organization of design information

2. *Documenting information with the tool is disruptive to my work

3. The tool could support collaborative building of design knowledge in my project team

4. *The effort I spend using the tool is worth the benefits

5. It makes the things I want to accomplish clearer and easier to get done.

6. The tool contains features that support discussions with my fellow designers or with others

7. Overall, I expect the tool to be useful in my organization

Figure 11-6.4 and Figure 11-6.5 show the box plots of evaluation scores from Indes and Philips respectively.
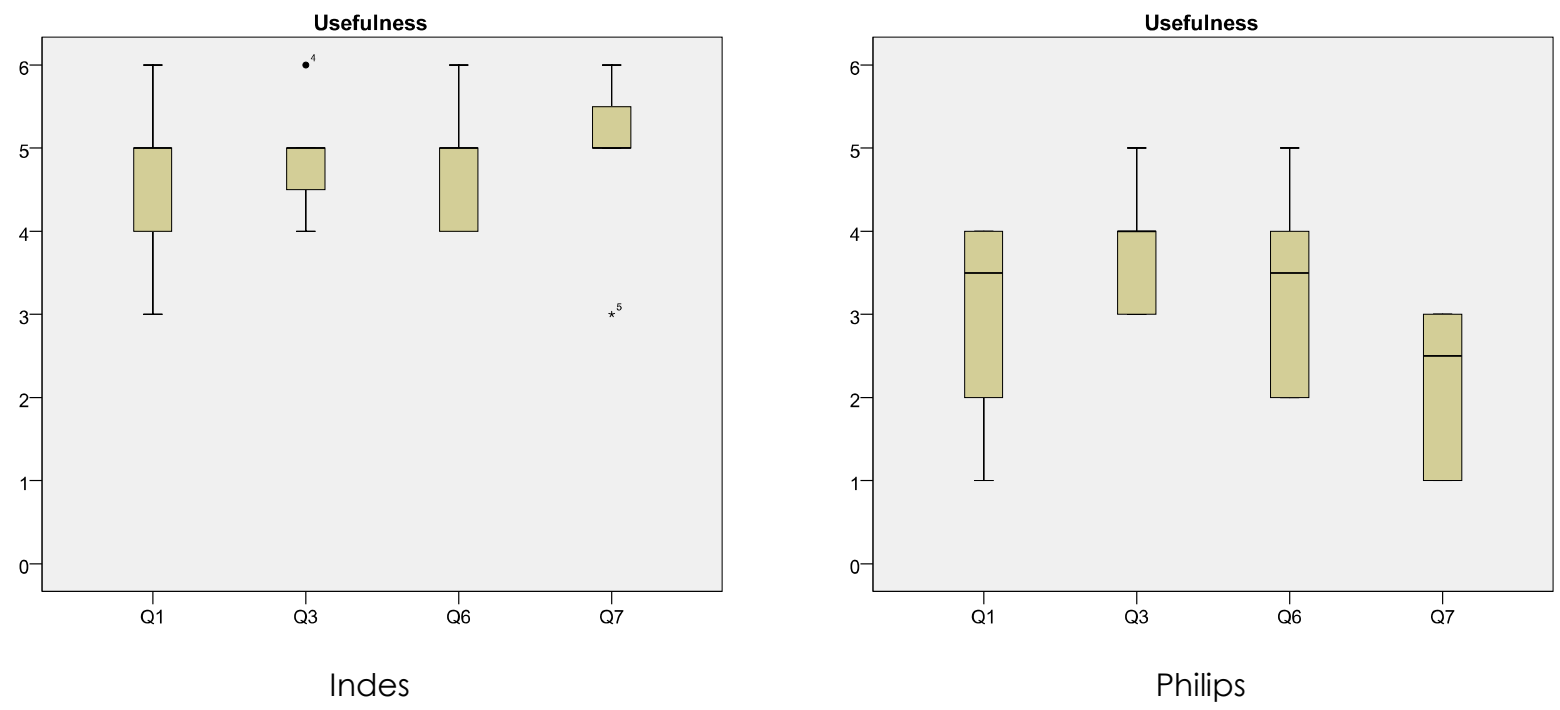

Figure 11-6.4: Detailed comparison of usefulness scores between Indes and Philips (part 1).

Questions 1, 3, 6 and 7 deal with the usefulness of the support tool on the organizational level. Figure 11-6.4 clearly shows that Indes designers find that the tool would be useful in their organization, more than Philips Application Researchers do. It is noteworthy to mention that the results of Philips are representative only for the ARC department.
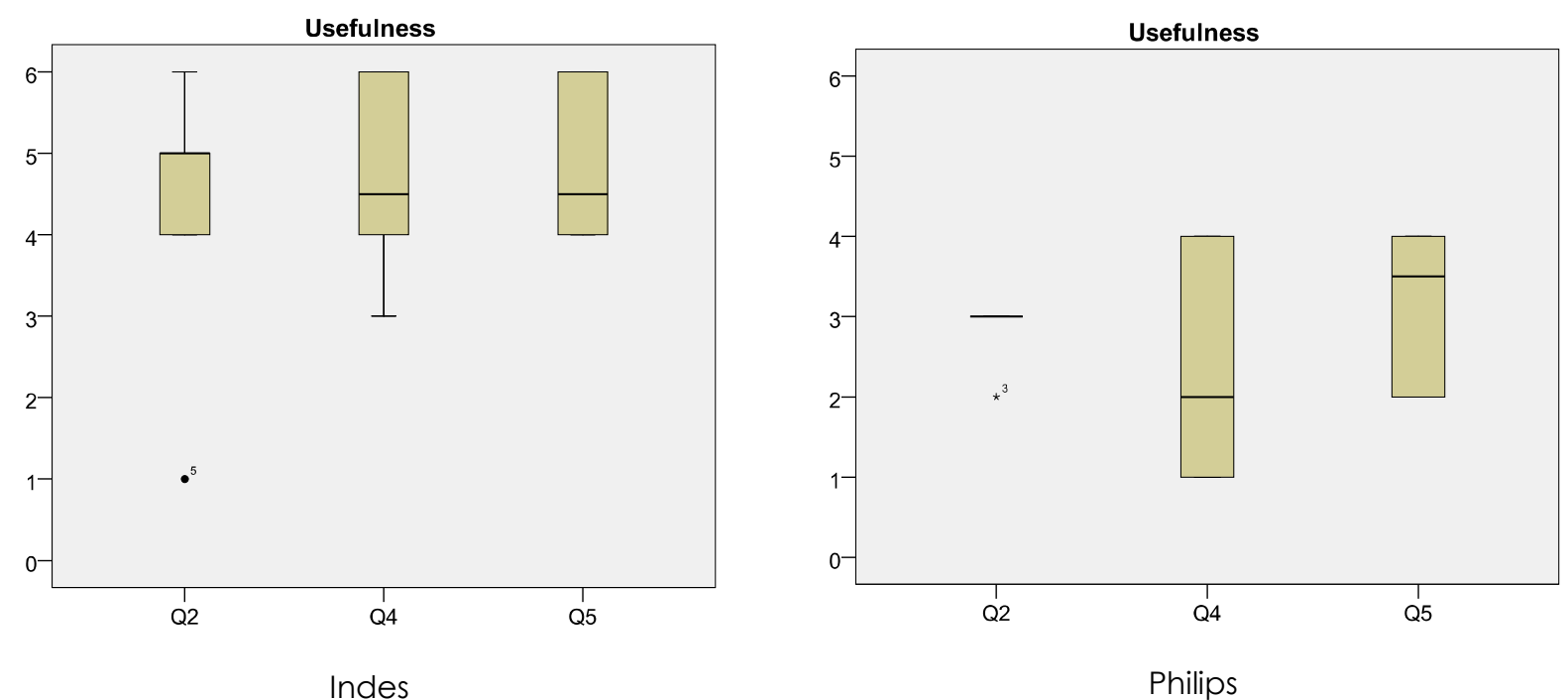

Figure 11-6.5: Detailed comparison of usefulness scores between Indes and Philips (part 2). 
Questions 2, 4 and 5 concern each participant's work ritual and individual acceptance towards the tool. While the questions reflect on the individuals, the responses are largely influenced by the organization's approach. For instance, Indes makes scenarios an integral part in their design projects. The designers are exposed to scenario-based design and therefore could conclude that the support tool, which supports their practice, would be useful.

\section{Satisfaction}

The satisfaction category consists of seven questions as the following:

1. I would recommend it to a colleague.

2. It is fun (engaging, not boring) to use.

3. I found it very cumbersome (slow, or complicated) to use.

4. It accommodates the flow of my thinking process during analysis, synthesis and evaluation phases.

5. It helps me to be creative (imaginative).

6. I expect that I would use the tool frequently.

7. Overall, I am satisfied with the way the tool works.

Figure 11-6.6 and Figure 11-6.7 show the box plots of evaluation scores from Indes and Philips respectively.
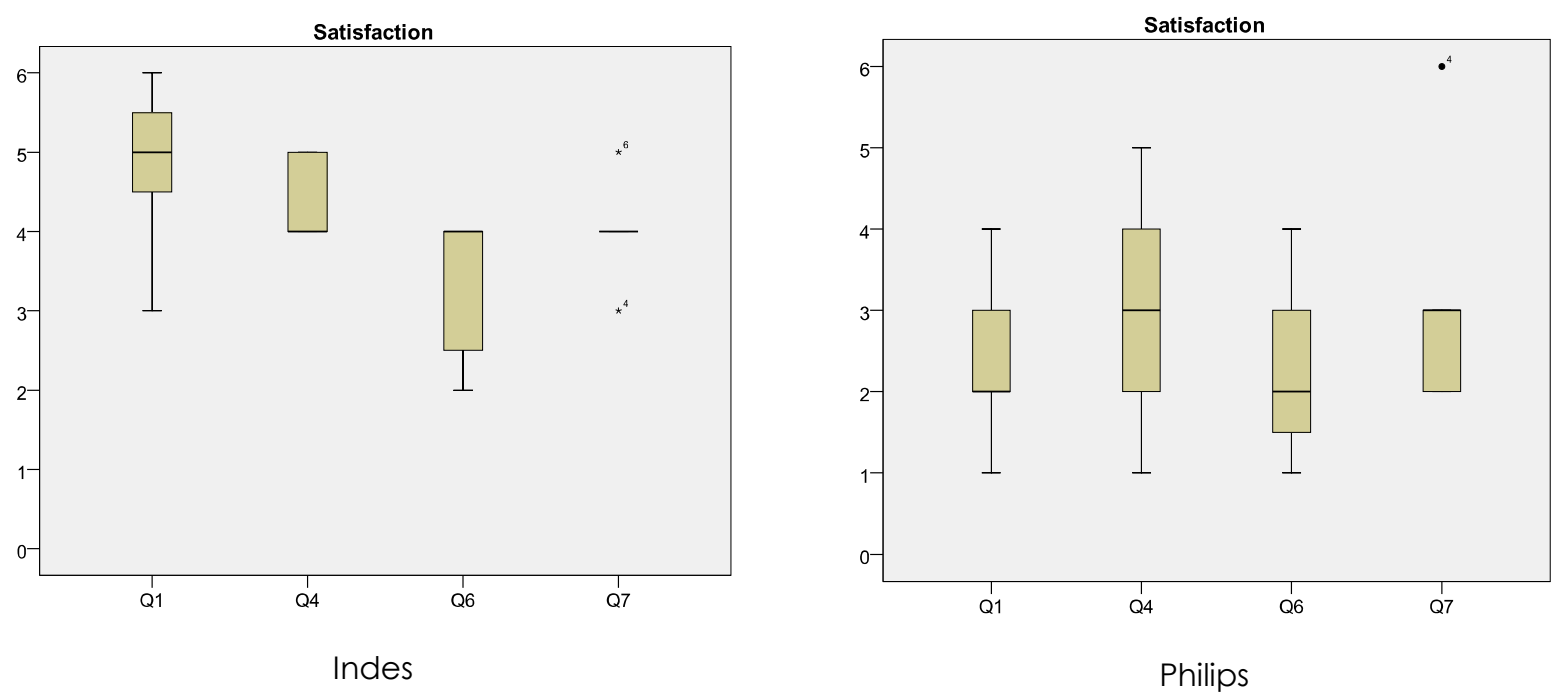

Figure 11-6.6: Detailed comparison of satisfaction scores between Indes and Philips (part 1).

Questions 1, 4, 6 and 7 attempt to probe into the participants' personal satisfaction and level of commitment, which are influenced by how useful the tool in helping them do their work. While this category is more appropriate for the real implementation of the support tool, it now serves as an indication. In this sense, question 1 "I would recommend it to a colleague" matters more than the other questions, as it implies enthusiasm about the idea presented in the tool. In the case of Philips, due to the complex organization structure, the term 'colleague' is a bit unclear. The tool is not relevant with the practice of ARC; however, it is unknown whether other departments would find it interesting. 

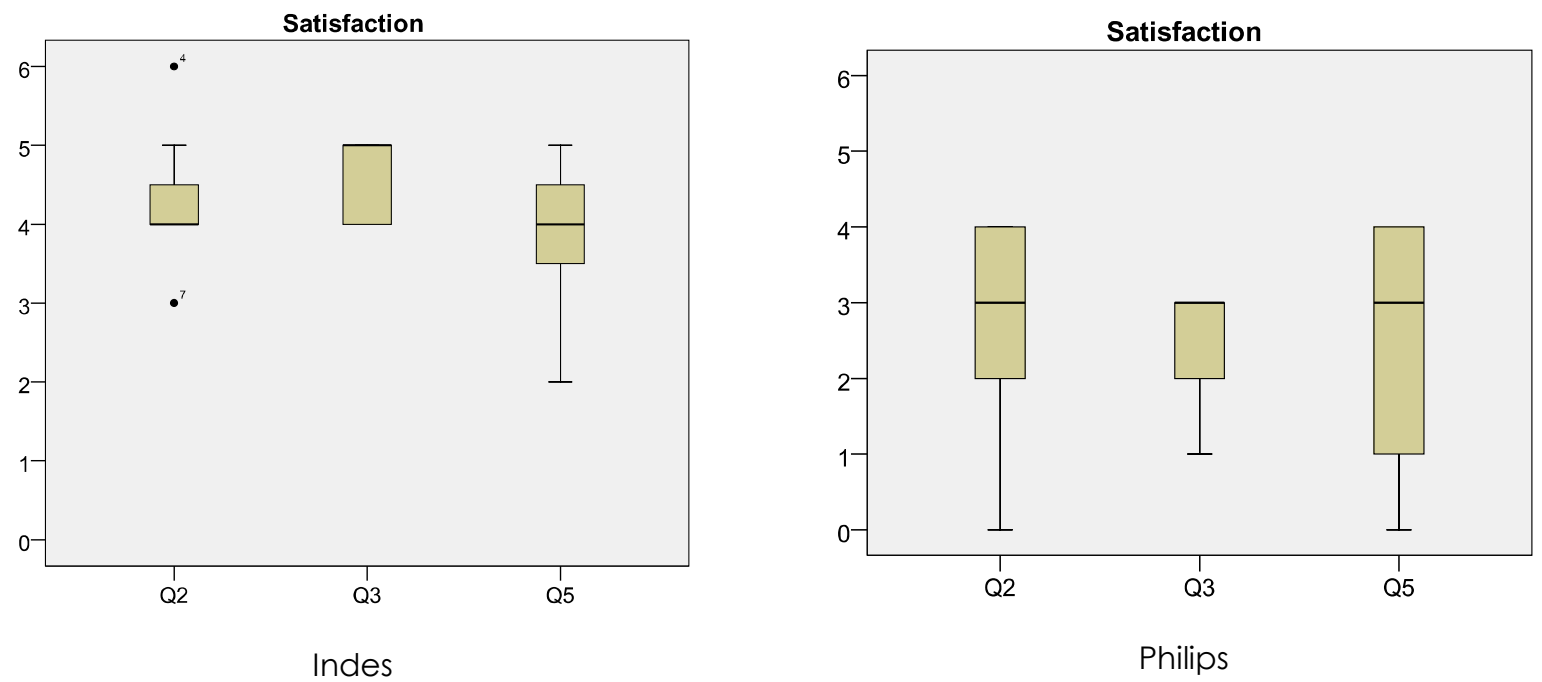

Figure 11-6.7: Detailed comparison of satisfaction scores between Indes and Philips (part 2).

Questions 2, 3 and 5 checks whether the tool has desirable attributes that would motivate and engage the users. Relating with the findings in the previous section, more visuals - to be used with the informal approach of the tool- would improve the satisfaction in using it considerably. The visualization of information, however, is not the focus of this research and therefore not implemented in the prototype.

\section{How can the tool be improved?}

Lastly, this category asks direct questions to probe for desirable/needed features.

1. The tool should give a more elaborate overview/summary of the documented information.

2. The tool should provide a more visual overview (in addition to the current textual/list overview).

3. The tool should provide more types of output that will be used in other design activities in the project.

Figure 11-6.8 shows the box plots of evaluation scores from Indes and Philips respectively.
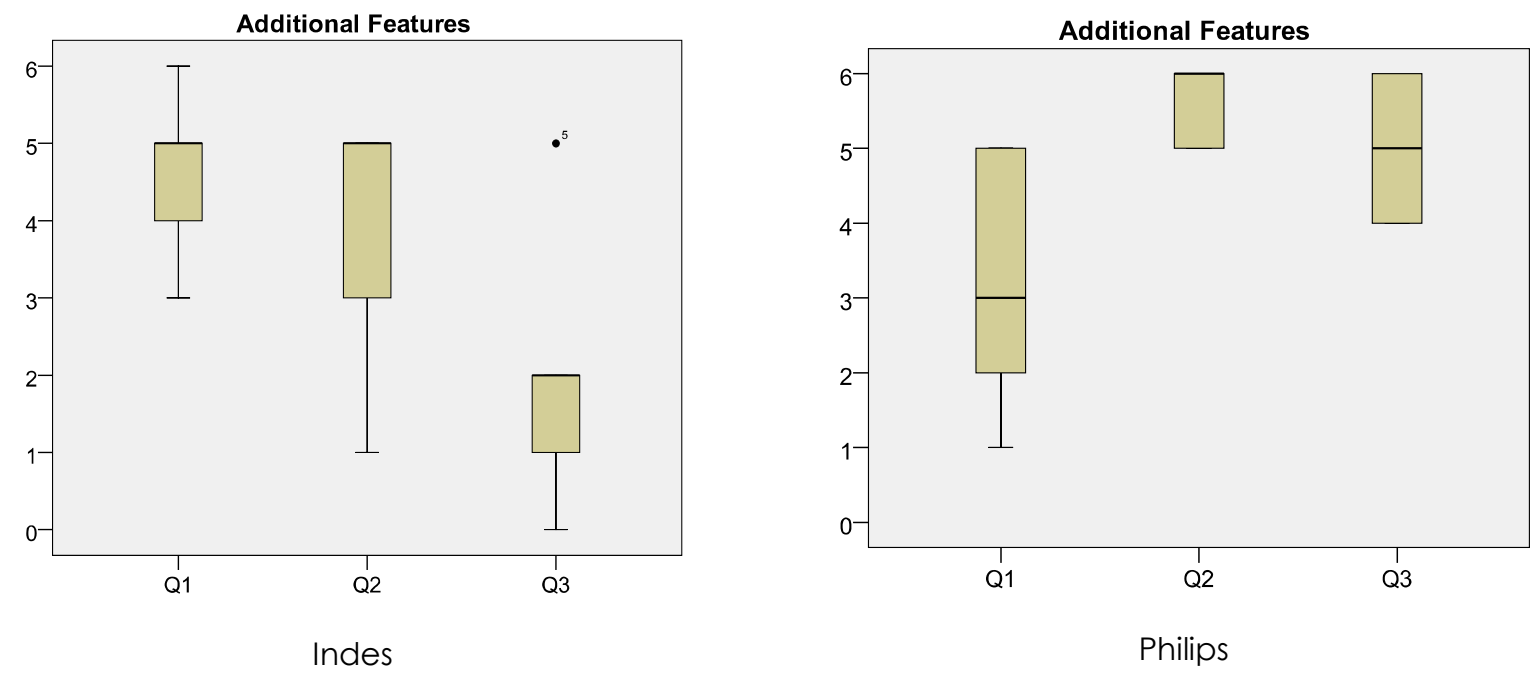

Figure 11-6.8: Detailed comparison on the scores of proposed additional features between Indes and

Philips.

These questions reconfirm that there is room for further development, especially additional functionality that builds on the basic one. At Indes, the designers prefer to keep the tool simple by not adding more functionality to avoid the tool becoming too complex for their lean 
practice. Their scores on question 3 reflect this. On the other hand, the high scores from Philips indicate that the tool needs to mature much more before it can be useful in their organization. Furthermore, the Philips ARC has their information covered by another system, and therefore there is a variable opinion about whether "a more elaborate summary of information" is necessary (question 1). Their responses show that visual overview is strongly preferred. 


\section{Appendix 7 Future Scenarios}

Two variables, company culture and target market, are recognized as influencing factors for the application of the support tool. Within the frame of these two variables, four scenarios have been developed to illustrate plausible applications of the support tool.

"Big Company" culture
Specialized functions, clearly defined input-
output of each function, less transparency
(more bureaucracy), longer process, legacy
system. Interfacing/integration of results is
crucial.

Global Market

Technology-push, customizable generic solutions, outsourcing, global business partners, identification

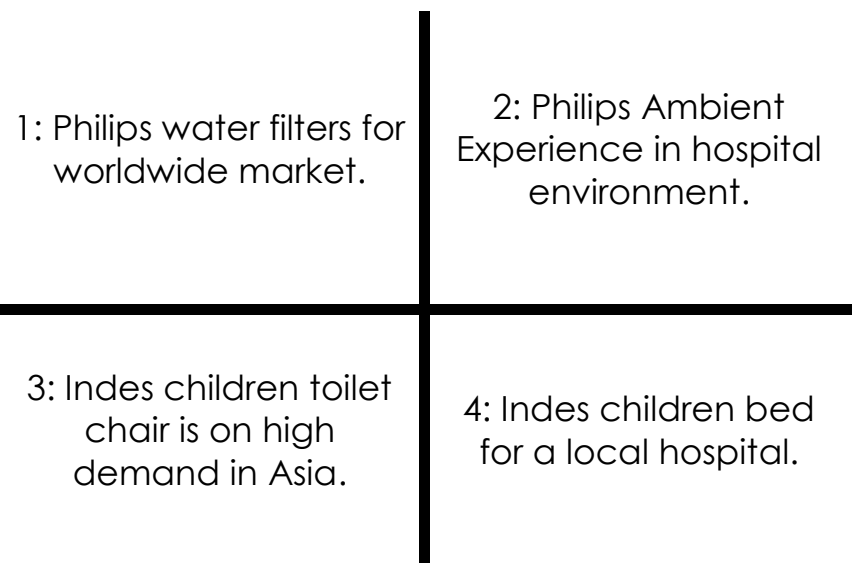
Specific Market Identified/local market, centralized process loutsourcing is new markets, wider range of users. User research and team communication is crucial.

\section{"Small Company" culture}

All-round designers, flexible functions, more transparency (less bureaucracy), time-pressure, a lean use of formal tools. Responsibility and contribution in the team is crucial.

Figure 11-7.1: The axes represent the most influential factors that shape the design approach; the four areas highlight the potential roles of the support tool in the different contexts.

The following scenarios flesh out the potential roles of the support tool in fictive stories. The company names and the cases are genuine, though the project and client names are made up. The rest of the stories however, is not factual and only serves to portray the hypothetical situations in the presence of the support tool during the mentioned projects.

\section{Scenario 1: Philips water filters for worldwide market.}

Royal Philips Electronics of the Netherlands is a diversified Health and Well-being company, focused on improving people's lives through timely innovations. As a world leader in healthcare, lifestyle and lighting. Philips integrates technologies and design into people-centric solutions, based on fundamental customer insights and the brand promise of "sense and simplicity".

With environment issues looming in the everyday life of people anywhere, a concern about clean water is growing in the society. While it was initially more apparent in poor countries, nowadays people in developed countries also prefer to filter their drinking water. Philips has been leading in this domain for quite some time and is ready to address a more global market. With international branches in different parts of the world, teams are formed and assigned to conduct user research to better fit the water filter solution to each specific market. Despite the distance, the teams can collaborate by sharing their findings on the same online platform, Scenario Central. The user data and scenarios are grouped based on specific keywords and other demographic details, so that they are easily searchable. With the same platform, the teams can follow each other's progresses, and sometimes be stimulated by the diversity of the findings. 
The designers in the design department follow the results from user research closely also using Scenario Central. The information about the various types of users in different locations, ways of life, contexts, concerns, motivations, gives them an overview of how their standard water filter can be adjusted to address different markets. With each concept, the designers compose hypothetical use scenarios, specifically mentioning the user type they design it for. Their colleagues (a.o. the user researchers) can reflect on the concepts, often evaluating them together with the real target users.

Source of inspiration: http://www.philips.com/about/company/companyprofile.page

\section{Scenario 2: Philips Ambient Experience in hospital environment.}

Royal Philips Electronics of the Netherlands is a diversified Health and Well-being company, focused on improving people's lives through timely innovations. As a world leader in healthcare, lifestyle and lighting. Philips integrates technologies and design into people-centric solutions, based on fundamental customer insights and the brand promise of "sense and simplicity".

Philips Healthcare actively promotes the concept of a patient-friendly, people-focused hospital environment using ambient technologies. Ambient Experience Design is a customized solution that can be applied to departments, floors and even facilities in their entirety or partially in a specific hospital. The Ikhlas Sanubari Medical Center(ISMC) in Abu Dhabi has asked Philips to design the Ambient Experience Interventional Suite in the Department of Cardiac Sciences. This will be the first of its kind in the Middle East.

The user researchers in the team spend some time at ISMC in Abu Dhabi to absorb information about the hospital space and their users (doctors, nurses, patients). With the designers back in the headquarters, communication between the user researchers and the designers is crucial. The user researchers use Scenario Central to share their findings simultaneously online - there is little time to write formal documents. Based on these findings, the designers can start sketching and make impressions of the new hospital space with ambient technologies installment. It is especially essential to understand how cultural and ethnic differences impact the acceptance of the Ambient Experience environment. The users in the Middle East have different perspectives, e.g. about patient modesty, that are not familiar with the designers. The user researchers create elaborate user profiles on Scenario Central that inform the designers of these differences. Additionally, they also compose scenarios that highlight the different reactions of the users in various use situations. The designers take this challenge well. For instance, they innovatively address the issue of patient modesty through the use of Privacy Glass. Privacy Glass separates the spaces in the scanning room and can be switched from clear to opaque at the touch of a button, assuring the utmost patient privacy.

Impressed with the good work of Philips, ISMC wants to learn from the design process conducted by the design team. ISMC, as the leading medical provider in Abu Dhabi, wishes to use the usercentred knowledge from Philips to attract other foreign collaborators and investors so they could extend the Ambient Experience in other departments. Philips already has the information organized: the user profiles and different scenarios (online on Scenario Central), and the technical specifications of the solution (internal). Therefore, Philips easily manages the partial information that can be released to showcase their Ambient Experience solutions. The general information about users and scenarios serves as a case study on creating Ambient Experience for users from different cultures.

Source of inspiration:

http://www.philips.com/about/company/companyprofile.page

http://www.healthcare.philips.com/main/products/ambient_experience/clinical_solutions/dept_design/in dex.wpd

\section{Scenario 3: Indes children toilet chair is on high demand in Asia.}

Indes, a Dutch design agency, is a small/medium sized company of about 35 employees in The Netherlands and 20 employees in China. They pride themselves for being agile and flexible in their user-focused approach. The designers are versatile as they are trained to be all-round in different functions. Throughout the years Indes has developed a good reputation within The Netherlands. 
Indes' business partner in China, SHOTO, has identified a need for a shower-and-toilet chair for handicapped children. Realizing also its relevance for European market, Indes works hand-inhand with SHOTO to define the problem in detail, research the target users, and formulate requirements. This collaboration takes place on the Scenario Central platform. It provides a framework for both teams for a coherent and consistent documentation of their results. The transparency makes sharing the research data easier; every team member knows where the progress is at despite the distance. The result is a product that fits in well with the children's world of perception, easy to use by the parents/caretakers, and safe.

To translate the product to the Chinese market, additional user research is conducted by SHOTO. Different setting, culture, language, user physiology add extra specific requirements. These data is also documented on Scenario Central, using the earlier data as a basis. The user research data will certainly be useful for future projects, especially with markets in different parts of the world.

Source of inspiration: http://www.indes.eu/Product.aspx?Product=56

\section{Scenario 4: Indes designs children bed for a local hospital.}

Indes, a Dutch design agency, is a small/medium sized company of about 35 employees in The Netherlands and 20 employees in China. They pride themselves for being agile and flexible in their user-focused approach. The designers are versatile as they are trained to be all-round in different functions. Throughout the years Indes has developed a good reputation within The Netherlands.

A new hospital is being opened in the region. Indes will design the hospital bed for children. Users are involved early in the product design process. The design team develops contacts with nurses, cleaners, other caretakers, parents to get their experiences and knowledge for inspiration. The team uses Scenario Central to create profiles of these real people. Users' domain knowledge is translated into scenarios and requirements, which are documented and related with the user profiles. By actively involving users, Indes successfully delivers a bed design with better functionality, high acceptance, and low-cost production. Indes also sets a new "standard" for safety in hospital furniture design, which previously has not been defined.

Satisfied with the bed design, the hospital gives a new assignment to Indes to design hospital lockers/cabinet. With the same users and setting, the newly-composed team can simply reuse the existing data on Scenario Central. The system automatically registers who contributes what, so that new members can easily find the person responsible for the data for questions/clarification.

From these projects with the hospital, Indes has developed reputation as the leading expert on hospital furniture safety. Other design companies start requesting advices to Indes. An extension to the Scenario Central enables publishing of selected data, searchable online by other parties. With the rationales and sources intact, this online database of knowledge generates a lot of interests. Recognizing the business value of this opportunity, Indes creates a service in which other parties can subscribe to the database. An addition to the Scenario Central platform allows access management for different types of subscription.

Source of inspiration: http://www.indes.eu/Product.aspx? Product=18 


\section{References}

Alexander, I. F. and N. Maiden, Eds. (2004). Scenarios, Stories, Use Cases: Through the Systems Development Life-Cycle, John Wiley \& Sons, Ltd.

Ambler, S. W. (2001-2009). "Reuse in Use-Case Models: <<extend $>>,<<$ include>>, and Inheritance." Excerpt from Chapter 5 of Ambler, S.W. (2004). The Object Primer 3rd Edition: Agile Model Driven Development with UML 2. Cambridge University Press. Retrieved July 21, 2010, from http://www.agilemodeling.com/essays/useCaseReuse.htm.

Anggreeni, I. and M. C. van der Voort (2008a). "Tracing the Scenarios in Scenario-Based Product Design: A Study to Understand Scenario Generation." Design Principles and Practices: An International Journal 2(4): 123-136.

Anggreeni, I. and M. C. van der Voort (2009a). "Bringing Scenario-Based Product Design into Practice: A Framework for Building and Using Scenarios." Design Principles and Practices: An International Journal 3(2): 339-354.

Anggreeni, I. and M. C. van der Voort (2009b). Supporting Scenario-Based Product Design and Its Adapters: An Informal Framework for Scenario Creation and Use. $\mathrm{HCl}$ International, San Diego, USA, Springer.

Anggreeni, I. and M. C. van der Voort (2009C). Supporting Scenario-Based Product Design: the first proposal for a scenario generation support tool. 19th CIRP Design Conference, Cranfield, UK, Cranfield University Press.

Anggreeni, I. and M. C. Van der Voort (2008b). Classifying Scenarios in a Product Design Process: a study towards semi-automated scenario generation. Proceedings of CIRP Design Conference 2008: Design Synthesis, University of Twente, Enschede, The Netherlands.

Antón, A. I. and C. Potts (1998). "A Representational Framework for Scenarios of System Use." Requirements Engineering 3(3): 219-241.

Bevan, N. (1999). "Quality in use: meeting user needs for quality." Journal of Systems and Software 49(1): 89-96.

Blomquist, A. and M. Arvola (2002). Personas in Action: Ethnography in an Interaction Design Team. NordiCHI, Aarhus, Denmark, ACM Press.

Bodker, S. (2000). "Scenarios in user-centred design - setting the stage for reflection and action." Interacting with Computers 13(1): 61-75.

Brouwer, M. and M. C. van der Voort (2006). "Design for Dynamic Use Situations." In Proceedings of Wonderground 2006 Design Research Society International Conference.

Burge, S. (2010). Retrieved Feb 1, 2010, from http://www.alledia.com/blog/generalcms-issues/joomla-and-drupal-version-2/.

Cambridge. (2010). "Cambridge Advanced Learner's Dictionary." Retrieved March 12, 2010, from http://dictionary.cambridge.org.

Campbell, R. L. (1992). "Will the real scenario please stand up?" SIGCHI Bulletin 24(2): 68.

Carroll, J. M. (2000a). "Five reasons for scenario-based design." Interacting with Computers 13(1): 43-60.

Carroll, J. M. (2000b). Making Use: Scenario-Based Design of Human-Computer Interactions. London, MIT Press.

Carroll, J. M., Ed. (1995). Scenario-based Design: Envisioning Work and Technology in System Development. New York, USA, John Wiley and Sons.

Cooper, A. (1999). The Inmates are Running the Asylum, Macmillan. 
Cooper, A. and R. Reimann (2003). About Face 2.0: The Essentials of Interaction Design, Wiley Publishing, Inc.

Cooper, R. G. and E. J. Kleinschmidt (1986). "An Investigation into the New Product Process: Steps, Deficiencies, and Impact." Journal of Product Innovation Management 3(2): 71-85.

CREWS. (1999). "CREWS: Cooperative Requirements Engineering with Scenarios." Retrieved March 1, 2010, from http://sunsite.informatik.rwth-aachen.de/CREWS/.

Cross, N. (1994). Engineering design methods: strategies for product design, John Wiley \& Sons.

DeBono, E. (1990). Lateral Thinking: Creativity step-by-step. New York, Harper-Collins.

Dorst, K. (2008). "Design research: a revolution-waiting-to-happen." Design Studies 29: 411.

Drupal. (2010a). "Drupal." Retrieved Feb 1, 2010, from http://www.drupal.org.

Drupal. (2010b). "Drupal Handbooks." Retrieved Feb 1, 2010, from http://drupal.org/handbooks.

Ehn, P. (1993). Scandinavian design: on participation and skill. Participatory Design, Principles and Practices. D. Schuler and A. Namioka, Lawrence Erlbaum.

Floyd, C. (1988). "A paradigm change in software engineering." SIGSOFT Software Engineering Notes 13(2): 25-38.

Fulton Suri, J. and M. Marsh (2000). "Scenario building as an ergonomics method in consumer product design." Applied Ergonomics 31 (2): 151-157.

Geller, T. (2008). "Drupal Essential Training (Chapter 1-2)." Retrieved Feb 1, 2010, from http://www.lynda.com/home/DisplayCourse.aspx?lpk2=620.

Haumer, P., K. Pohl and K. Weidenhaupt (1998). "Requirements elicitation and validation with real world scenes." IEEE Transactions on Software Engineering, Special Issue on Scenario Management 24(12): 1036 - 1054.

Hobbs, R. L. and C. Potts (1998). Towards a Framework for Hypermedia Scenarios. College of Computing Technical Report. Atlanta, GA, Georgia Institute of Technology.

Hobbs, R. L. and C. Potts (2000). Hyperscenarios: a framework for active narrative. 38th annual on Southeast regional conference, Clemson, South Carolina, ACM Press.

ISO (1998). ISO 9241 - 11 Ergonomic requirements for office work with visual display terminals (VDTs) - Part 11: Guidance on Usability. Geneva, Switzerland, International Organization for Standardization.

ISO/IEC (2001). ISO/IEC 91261 Software engineering - Product quality - Part 1: Quality model. Geneva, Switzerland, International Organization for Standardization.

ISTAG (2001). Scenarios for Ambient Intelligence in 2010. K. Ducatel, M. Bogdanowicz, F. Scapolo, J. Leijten and J.-C. Burgelman, ISTAG (Information Society Technologies Advisory Group).

Jacobson, I. (1995). The Use-Case Construct in Object-Oriented Software Engineering. Scenario-based design: envisioning work and technology in system development J. M. Carroll, John Wiley \& Sons, Inc.

Jacobson, I., M. Christerson, P. Jonsson and G. Overgaard (1991). Object Oriented Software Engineering a Use Case Driven Approach, Addison-Wesley.

Jarke, M., J. Bubenko, C. Rolland, A. Sutcliffe and Y. Vassilou (1993). Theories underlying requirements engineering: an overview of NATUREat Genesis. Requirements Engineering, 1993., Proceedings of IEEE International Symposium on, San Diego, CA, USA. 
Jarke, M., X. T. Bui and J. M. Carroll (1998). "Scenario Management: An Interdisciplinary Approach." Requirements Engineering 3(3): 155-173.

Jones, J. C. (1981). Design Methods. Chichester, Wiley.

Joomla. (2010). "Joomla." Retrieved Feb 1, 2010, from http://www.joomla.org.

Jordan, P. W. (1997). "Putting the pleasure into products." IEE Review 43(6): 249-252.

Karat, J. (1995). Scenario use in the design of a speech recognition system Scenariobased design: envisioning work and technology in system development J. M. Carroll, John Wiley \& Sons, Inc.: 109-133

Kruger, C. and N. Cross (2006). "Solution driven versus problem driven design: strategies and outcomes." Design Studies 27(5): 527-548.

Kuutti, K. (1995). Work Processes: Scenario as a Preliminary Vocabulary. Scenario-based Design. J. M. Carroll, John Wiley and Sons, Inc.

Leite, J. C. S. d. P., G. Hadad, J. Doorn and G. Kaplan (2000). "A Scenario Construction Process." Requirements Engineering 5(1): 38-61.

Lim, Y. and K. Sato (2006). "Describing Multiple Aspects of Use Situation: Applications of Design Information Framework to Scenario Development." Design Studies 27(1): 57-76.

Lincoln. (2008). "45 Drupal Sites Which You May Not Have Known Were Drupal Based." Retrieved Feb 1, 2010, from http://socialcmsbuzz.com/45-drupal-sites-which-youmay-not-have-known-were-drupal-based-24092008/.

Maiden, N. (1998). "CREWS-SAVRE: Scenarios for Acquiring and Validating Requirements." Automated Software Engg. 5(4): 419-446.

Microsoft. (2010). "What is Microsoft Surface?" Retrieved Feb 17, 2010, from http://www.microsoft.com/surface/Pages/Product/Whatls.aspx.

Moggridge, B. (1993). "Design by story-telling." Applied Ergonomics 24(1): 15-18.

Muller, M. J. and S. Kuhn (1993). "Participatory design." Communications of the ACM 36(6): 24-28.

Muller, M. J., D. M. Wildman and E. A. White (1993). "Taxonomy of PD practices: A brief practitioner's guide." Communications of the ACM 36 (4): 25-28.

Nardi, B. A. (1992). "The use of scenarios in design." SIGCHI Bulletin 24(4): 13-14.

Nielsen, J. (1990). Paper versus computer implementations as mockup scenarios for heuristic evaluation. Proceedings of the IFIP TC13 Third International Conference on Human-Computer Interaction, North-Holland Publishing Co., Amsterdam, The Netherlands.

Nintendo. (2010). "What is Wii?" Retrieved Feb 17, 2010, from http://www.nintendo.com/wii/what.

Pahl, G. and W. Beitz (1996). Engineering Design: A systematic approach. London, Springer Verlag Ltd.

Penna, G. D., B. Intrigila, A. R. Laurenzi and S. Orefice (2003). "An XML Definition Language to Support Scenario-Based Requirements Engineering." International Journal of Software Engineering and Knowledge Engineering (IJSEKE) 13(3): 237256.

Penna, G. D., B. Intrigila, A. R. Laurenzi and S. Orefice (2006). "An XML environment for scenario based requirements engineering." Journal of Systems and Software 79(3): 379-403.

Potts, C. (1995). Using schematic scenarios to understand user needs. In Proceedings of the Symposium on Designing Interactive Systems: Processes, Practices, Methods and Techniques, Ann Arbor, Michigan, United States, ACM Press.

Propp, V. (1958). Morphology of the folktale. The Hague, Mouton. 
Pruitt, J. and J. Grudin (2003). Personas: practice and theory. Proceedings of the 2003 conference on Designing for user experiences, San Francisco, California, ACM Press.

Robertson, S. (2004). Scenarios in Requirements Discovery. Scenarios, Stories, Use Cases: Through the Systems Development Life-Cycle. I. F. Alexander and N. Maiden, John Wiley \& Sons, Ltd.: 39-59.

Rolland, C. and C. B. Achour (1997). "Guiding the construction of textual use case specifications." Data and Knowledge Engineering 25(1): 125-160.

Rolland, C., C. B. Achour, C. Cauvet, J. Ralyté, A. Sutcliffe, N. Maiden, M. Jarke, P. Haumer, K. Pohl, E. Dubois and P. Heymans (1998a). "A proposal for a scenario classification framework." Requirements Engineering 3(1): 23-47.

Rolland, C., C. Souveyet and C. B. Achour (1998b). "Guiding goal modelling using scenarios." IEEE Transactions on Software Engineering, Special Issue on Scenario Management 24(12): 1055-1071.

Ronkko, K., M. Hellman, B. Kilander and Y. Dittrich (2004). Personas is not Applicable: Local Remedies Interpreted in a Wider Context. Participatory Design Conference, Toronto, Canada, ACM Press.

Rosson, M. B. and J. M. Carroll (2002). Usability Engineering: Scenario-based Development of Human-Computer Interaction. San Francisco, Morgan-Kaufman.

Sanders, E. B.-N. (1992). "Converging Perspectives: Product Development Research for the 1990s." Design Management Journal 3(4).

Schwartz, P. (1996). The Art of the Long View: Planning for the Future in an Uncertain World, Currency.

Shigley, J. E. and C. R. Mischke (1986). Standard handbook of machine design. New York, McGraw-Hill Inc.

Shin, J. E., A. G. Sutcliffe and A. Gregoriades (2005). "Scenario advisor tool for requirements engineering." Requirements Engineering 10(2): 132-145.

Svanaes, D. and G. Seland (2004). Putting the Users Center Stage: Role Playing and Low-fi Prototyping Enable End Users to Design Mobile Systems. SIGCHI conference on Human factors in computing systems, Vienna, Austria, ACM Press.

Ullman, D. G. (1992). The mechanical design process. New York, McGraw-Hill Inc.

van der Bijl-Brouwer, M. and M. C. van der Voort (2008). "Designing for Dynamic Usability: Development of a Design Method that Supports Designing Products for Dynamic Use situations." Design Principles and Practices: An International Journal 2(1): 149-158.

van der Heijden, K. (2005). Scenarios: The Art of Strategic Conversation, John Wiley \& Sons, Ltd.

Weidenhaupt, K., K. Pohl, M. Jarke and P. Haumer (1998). "Scenarios in system development: current practice." Software, IEEE 15(2): 34-45.

Wendrich, R. E., H. Tragter, F. G. M. Kokkeler and F. J. A. M. van Houten (2009). Bridging the Design Gap: Towards an Intuitive Design Tool. Icsid World Design Congress. Singapore.

Wensveen, S. A. G. (1999). Probing experiences. The first international conference on Design and Emotion, Delft University of Technology.

Wright, P. (1992). "What's in a scenario?" SIGCHI Bulletin 24(4): 11-12. 



\section{About the Author}

Irene Anggreeni was born in Kudus, Central Java, Indonesia on 6 February 1982. She attended primary school (SD Keluarga) and junior high school (SMP Negeri I) in her hometown Kudus. She completed her high school (SMU Kolese Loyola) in a neighbouring town Semarang. During high school, she developed an interest for natural science and its broad applications in life. Though at the time she was also fascinated with computers, internet, Information Technology, and its industry, and wondered what more is

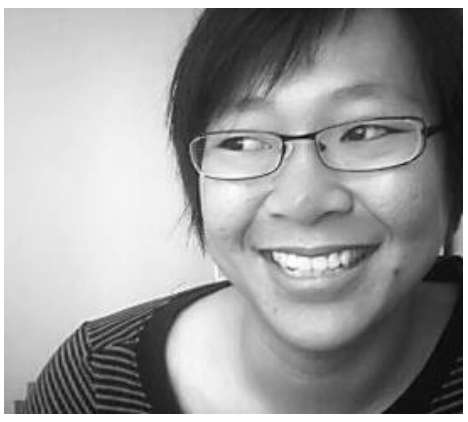
possible and can be achieved in this field. Thus, she decided to study Informatics for her bachelor degree in Bina Nusantara University in Jakarta. Upon graduation, she was working as a software engineer at PT Mitrais in Kuta, Bali during which she became attracted to the human aspect of software development. Actively searching for opportunities to further her education, she was soon accepted to study at Linkoping University, Sweden with scholarship from The Swedish Foundation for International Cooperation in Research and Higher Education (STINT). Her master study has allowed her to specialize in human-computer interaction and usability, demonstrated in her master thesis on the design of low-fi prototyping tool for interaction designers. Her interest in researching interaction design, design practice, and design in its broadest sense, motivated her to pursue a further research in this direction.

In September 2006, she started her PhD at the Laboratory of Design, Production and Management of the University of Twente. Briefly, her work deals with the applicability of concrete stories about product use - scenarios - in the design of consumer products. The thesis presented here, is a result of this work.

Next to her academic life, she is actively pursuing and spreading her passion in swing dancing. She initiated and continues to teach the Lindy Hop group in Enschede which at the moment mainly consists of students and PhDs of the University of Twente. 